\title{
Functional analysis of proteases in tracheal development of Drosophila
}

\author{
Dissertation \\ for the award of the degree \\ "Doctor rerum naturalium" \\ of the Georg-August-Universität Göttingen
}

within the doctoral program Genes and Development of the Georg-August University School of Science (GAUSS)

\author{
submitted by \\ Leonard Drees \\ from Vorwerk, Germany
}

Göttingen, January 2019 


\section{Thesis Committee:}

Prof. Dr. Reinhard Schuh

Research Group Molecular Organogenesis, Max Planck Institute for Biophysical Chemistry, Göttingen

\section{Prof. Dr. Jörg Großhans}

Department of Developmental Biochemistry, Medical School, Georg-August University Göttingen

\section{Prof. Dr. Ernst A. Wimmer}

Department of Developmental Biology, Georg-August University Göttingen

\section{Members of the Examination Board:}

\section{Referee: Prof. Dr. Reinhard Schuh}

Research Group Molecular Organogenesis, Max Planck Institute for Biophysical Chemistry, Göttingen

\section{$2^{\text {nd }}$ Referee: Prof. Dr. Jörg Großhans}

Department of Developmental Biochemistry, Medical School, Georg-August University Göttingen

\section{Further members of the Examination Board:}

\section{Prof. Dr. Ernst A. Wimmer}

Department of Developmental Biology, Georg-August University Göttingen

\section{PD Dr. Gerd Vorbrüggen}

Department of Developmental Biology, Georg-August University Göttingen

\section{Prof. Dr. Ahmed Mansouri}

Research Group Molecular Cell Differentiation, Max Planck Institute for Biophysical Chemistry, Göttingen

\section{Dr. Roland Dosch}

Department of Developmental Biochemistry, Medical School, Georg-August University Göttingen

Date of oral examination: 14.03.2019 


\section{Affidavit}

I hereby declare that I prepared the Dissertation "Functional analysis of proteases in tracheal development of Drosophila" on my own, with no other sources and aids than quoted.

Parts of this thesis are already published in:

Drees L, Königsmann T, Jaspers MHJ, Pflanz R, Riedel D, Schuh R. Conserved function of the matriptase-prostasin proteolytic cascade during epithelial morphogenesis. PLoS Genet. 2019; 15(1): e1007882.

doi: 10.1371/journal.pgen.1007882.

The authors contributed to Drees et al. (2019) as follows:

Tatiana Königsmann performed injections of fluorophore-labeled dextran into the haemocoels of $N p$ mutant, mega mutant and control embryos. Transmission electron microscopy imaging was conducted by Dietmar Riedel. Martin H. J. Jaspers initially identified $N p$, tpr and lint in an RNAi screening for genes that are essential for tracheal gas filling. All other experiments as well as data analysis and preparation of figures were done by myself. The manuscript was written by Reinhard Schuh and me, with support from Ralf Pflanz. 


\section{Table of contents}

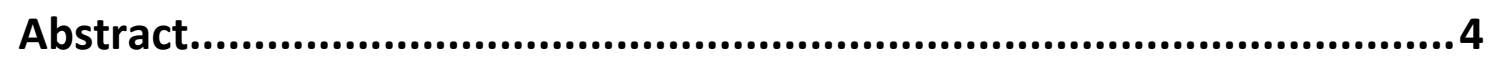

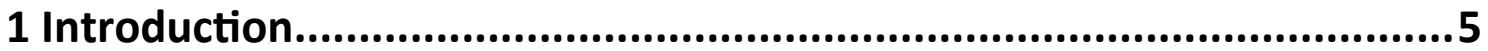

1.1 The tracheal system of Drosophila melanogaster...........................................5

1.1.1 Tracheal branching morphogenesis during embryogenesis........................................6

1.1.2 The role of transient apical extracellular matrix during tracheal development...............9

1.1.3 Tracheal cuticle formation and liquid clearance.....................................................12

1.2 The matriptase-prostasin proteolytic cascade.............................................16

1.2.1 Regulation and function of the matriptase-prostasin proteolytic cascade during

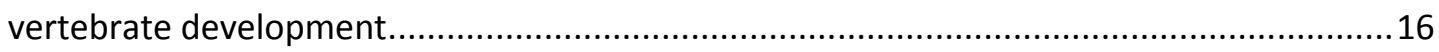

1.2.2 Role of the matriptase-prostasin proteolytic cascade in pathogenesis.......................20

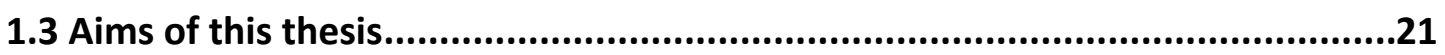

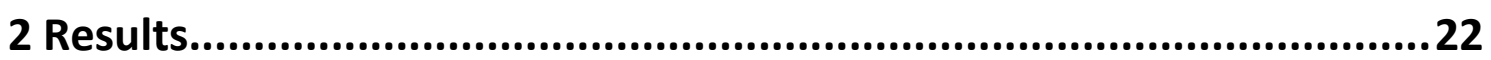

2.1 Functional analysis of Notopleural during embryogenesis.............................23

2.1.1 Notopleural encodes a type II transmembrane serine protease related to vertebrate matriptase.

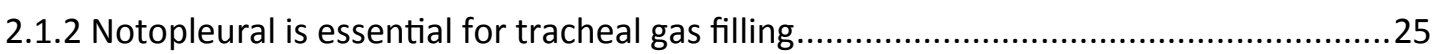

2.1.3 Notopleural is expressed in ectodermal tissues and localizes to apical plasma membranes and the apical extracellular matrix

2.1.4 Notopleural is essential for tracheal transepithelial barrier function but does not affect

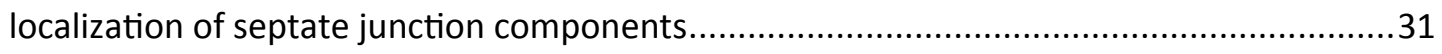

2.1.5 Tracheal epithelial barrier function maintenance is dependent on Notopleural...........34

2.1.6 Notopleural mediates tracheal apical extracellular matrix maturation.........................36

2.1.7 Notopleural is essential for epidermal cuticle organization......................................39

2.1.8 Intraluminal degradation of the zona pellucida domain protein Dumpy depends on Notopleural.

2.1.9 Notopleural regulates attachment of apical extracellular matrix to the apical plasma membranes in the tracheal system.

2.1.10 Release of the zona pellucida domain protein Piopio from the tracheal apical cell surface is mediated by Notopleural.

2.1.11 Localization patterns of Dumpy and Piopio in the epidermis depend on Notopleural

2.1.12 Proteolytic processing of Piopio is mediated by Notopleural.

2.1.13 Non-cell autonomous function of Notopleural during embryonic tracheal development.. 56

2.1.14 Function of Notopleural depends on its catalytic activity.......................................58

2.1.15 Notopleural zymogen activation is an autocatalytic mechanism...............................61

2.1.16 Human matriptase is functionally homologous to Notopleural................................63 
2.1.17 Piopio is proteolytically cleaved by Notopleural and matriptase.

2.1.18 Proteolytic cleavage of human transforming growth factor $\beta$ receptor type III is

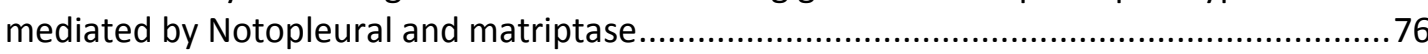

2.2 Functional analysis of CG4386 during embryogenesis...............................82

2.2.1 CG4386 encodes a trachea specific serine protease with sequence similarities to

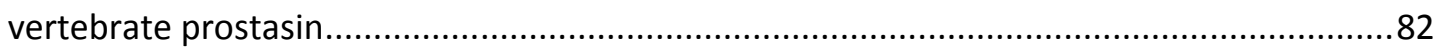

2.2.2 Tracheal-prostasin is essential for gas filling of the tracheal system ...........................84

2.2.3 Tracheal-prostasin localizes to the tracheal apical extracellular matrix.......................86

2.2.4 Tracheal network formation, transepithelial barrier function and epithelial polarization are not dependent on tracheal-prostasin

2.2.5 Tracheal-prostasin is required for attachment of the tracheal cuticle to tracheal cells and contributes to degradation and localization of Dumpy.............................................

2.2.6 Human prostasin is functionally homologous to Tracheal-prostasin............................94

2.3 Functional analysis of lumens interrupted during embryogenesis...................98

2.3.1 lumens interrupted encodes a type II transmembrane serine protease with sequence similarities to Notopleural.

2.3.2 lumens interrupted is essential for tracheal liquid clearance and transepithelial barrier function.

2.3.3 Lumens interrupted is expressed in embryonic ectodermal tissues and localizes to the tracheal lumen in late embryogenesis.

2.3.4 Lumens interrupted is required for tracheal intraluminal Dumpy degradation and

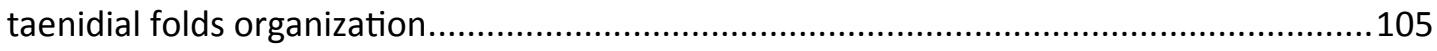

2.3.5 Genetic interactions of Lumens interrupted and Notopleural ................................107

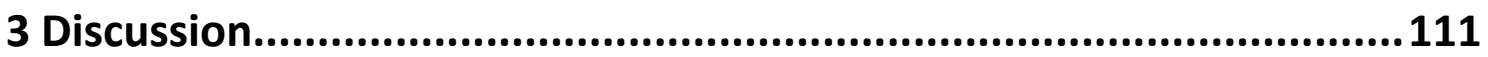

3.1 Tracheal apical extracellular matrix maturation is mediated by three serine proteases.

3.1.1 Non-cell autonomous initiation of tracheal apical extracellular matrix maturation by Notopleural.

3.1.2 Luminal apical extracellular matrix clearance - proteolytic teamwork for Dumpy degradation......

3.1.3 A proteolytic cascade drives formation of the tracheal cuticle taenidial folds

3.1.4 Multiple functions of Notopleural, Lumens interrupted and Tracheal-prostasin facilitate tracheal liquid clearance.

\subsection{Conserved function of the matriptase-prostasin proteolytic cascade during} epithelial morphogenesis.

3.2.1 Conserved role of the matriptase-prostasin proteolytic cascade in epithelial barrier function and liquid homeostasis regulation

3.2.2 Zona pellucida domain proteins as novel targets of the matriptase-prostasin proteolytic cascade.

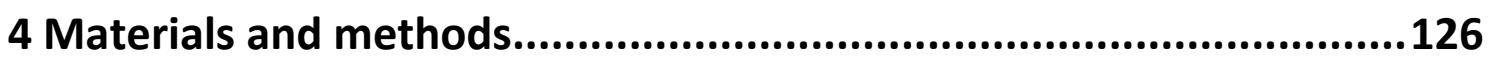

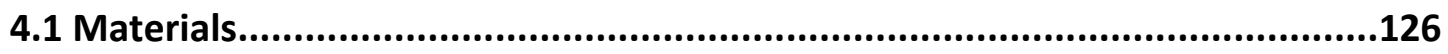

4.2 Fly handling, mutagenesis and transgenesis.............................................145

4.3 Analyses of embryos and larvae................................................................148 


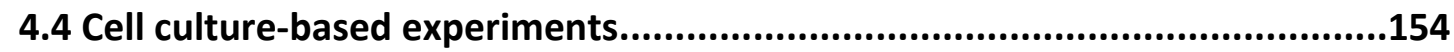

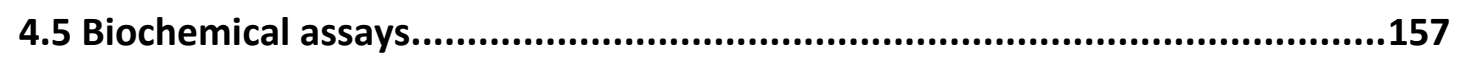

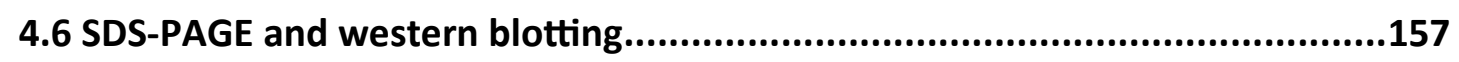

4.7 Light microscopy and image acquisition.....................................................158

4.8 DNA purification, amplification and molecular cloning..............................159

4.9 Software, algorithms and data repositories...........................................163

5 List of Abbreviations.............................................................164

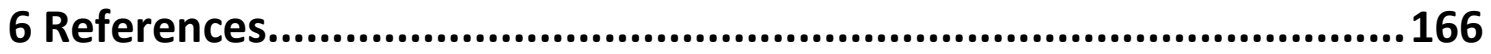

7 Appendix.........................................................................183

7.1 Synthesized double stranded DNA fragments..........................................183

7.2 Measurements of chitin strand diameters and frequencies.........................187

8 Acknowledgements..................................................................192

9 Curriculum vitae.........................................................................193 


\section{Abstract}

Formation and remodeling of extracellular matrix (ECM) structures is critical for epithelial development and organ morphogenesis. The apical ECM of epithelial tissues provides a scaffold for cell movements, contributes to intercellular signaling and acts as a protective barrier against pathogens and environmental factors in both, vertebrates and invertebrates. The type II transmembrane serine protease matriptase and the serine protease prostasin are key factors in a proteolytic cascade that regulates epithelial ECM differentiation during development in vertebrates. While apical ECM formation and remodeling was shown to be essential for Drosophila melanogaster organogenesis, mechanisms and pathways that regulate apical ECM dynamics largely remain elusive.

Here, I identify the D. melanogaster proteases Notopleural (Np) and Trachealprostasin (Tpr) as functional homologs of vertebrate matriptase and prostasin, respectively. Np mediates morphogenesis and remodeling of apical ECM during embryonic tracheal system development and is essential for maintenance of the transepithelial barrier function. Together with the protease Lumens interrupted (Lint), Np and Tpr mediate degradation of the zona pellucida domain (ZPD) protein Dumpy, which forms elastic fibers in the tracheal apical ECM via the conserved ZPD polymerization module. In addition, $\mathrm{Np}$, Lint and Tpr regulate the formation of the mature tracheal apical ECM cuticle. Furthermore, I demonstrate that Tpr zymogen activation is mediated by $\mathrm{Np}$ while zymogen activation of $\mathrm{Np}$ occurs in an autocatalytic manner, suggesting $\mathrm{Np}$ as an initiator of a proteolytic cascade in the tracheal system. Functional homology of $\mathrm{Np}$ and matriptase is based on a conserved substrate specificity, since both proteases cleave Tpr at its zymogen activation site, cleave the D. melanogaster ZPD protein Pio within its ZPD, and mediate cleavage of the human ZPD containing TGF-B receptor type III. The data therefore indicate that cleavage of ZPD proteins by the matriptase-prostasin proteolytic cascade is an evolutionary conserved mechanism. 


\section{Introduction}

\subsection{The tracheal system of Drosophila melanogaster}

The Drosophila melanogaster tracheal system is a well-established model for the development of branched tubular epithelial organs. It serves as a respiratory organ and supplies oxygen to various tissues. Epithelial tubes are a fundamental structure of metazoan tissues and found in mammalian organs like the kidneys, lungs, salivary glands, mammary glands and parts of the reproductive system, as well as in the D. melanogaster tracheal system, salivary glands and malphigian tubules (Maruyama and Andrew 2012; Andrew and Ewald 2010). The D. melanogastertracheal system emerged as a prime model for epithelial tube formation and branching morphogenesis due to the numerous genetic tools that are available in $D$. melanogaster, the relatively small time scale of tracheal organogenesis and the good accessibility of embryonic trachea for microscopic imaging. Research over the last decades revealed key mechanisms and signaling pathways that regulate differentiation, branching, growth and tube size in the $D$. melanogaster tracheal system (Hayashi and Kondo 2018; Zuo et al. 2013; Schottenfeld et al. 2010). While some aspects of D. melanogaster tracheal organogenesis are specific to invertebrates, many mechanisms of tracheal epithelial tube formation and branching morphogenesis were found to be conserved across invertebrates and vertebrates, likely representing fundamental principles of epithelial tubular organ development (Maruyama and Andrew 2012; OchoaEspinosa and Affolter 2012; Muñoz-Chápuli 2011).

After branching morphogenesis and network formation, the tracheal system of the D. melanogaster embryo fills with gas before hatching as larvae. Gas filling is essential for proper functioning of respiratory organs and occurs similarly in mammalian lungs and insect tracheal systems (Förster and Woods 2013; Hooper et al. 2009; Elias and O'Brodovich 2006). Prior to gas filling of the D. melanogaster embryonic tracheal system, extracellular matrix (ECM) depositions that are 
present in the tracheal lumen during development are removed and remodeled into a supracellular tracheal apical ECM (aECM) structure (Öztürk-Çolak et al. 2016a). In recent years, formation, degradation and remodeling processes of the tracheal aECM emerged as key regulators of tracheal organ size, shape and function (Dong and Hayashi 2015; Dong et al. 2014; Glasheen et al. 2010). However, underlying mechanisms and signaling pathways largely remain elusive. Furthermore, while formation and remodeling of aECM structures is also essential in development and pathogenesis of mammalian epithelial tubular organs, it is unclear whether basic principles of these processes are conserved across vertebrates and invertebrates (Chanda et al. 2018; Li et al. 2016; White 2015).

\subsubsection{Tracheal branching morphogenesis during embryogenesis}

Embryonic tracheal system development and branching morphogenesis have been studied extensively, leading to a detailed understanding of tracheal network formation and many underlying mechanisms (reviewed in Hayashi and Kondo 2018; Affolter and Caussinus 2008; Ghabrial et al. 2003). Embryonic development of $D$. melanogaster is divided into stages that are defined by specific morphological changes (Campos-Ortega and Hartenstein 1997). Tracheal development starts in stage 10 of embryogenesis with the differentiation of 10 tracheal cell clusters on each lateral side of the embryo. These so-called tracheal placodes contain $50-80$ cells each and their specification into tracheal cells is mainly driven by the transcription factor Trachealess (Trh; Isaac and Andrew 1996; Wilk et al. 1996;). Most notably, Trh regulates expression of the fibroblast growth factor (FGF) receptor tyrosine kinase encoding gene breathless (btl) which is essential for tracheal branching morphogenesis (Ohshiro and Saigo 1997). The tracheal placodes undergo invagination during stage 11 of embryogenesis and form tracheal pits. This process is mediated by acto-myosin driven apical constriction, Epidermal growth factor receptor (Egfr) signaling and Rho GTPase signaling (Brodu and Casanova 2006; Wilk et al. 1996). During the last steps of tracheal pit formation, the tracheal cells complete cycle 16 of mitosis after which cell division of tracheal cells is arrested until larval stages (Kondo and Hayashi 2013). Subsequent branching morphogenesis and growth of the embryonic tracheal system does therefore not depend on cell division. 
During stage 12, branches start to elongate from the sac-like tracheal pits. This process is guided by FGF signaling. The FGF receptor (Btl) expressing tracheal cells respond to the Branchless (Bnl) FGF ligand that is dynamically expressed and secreted by non-tracheal tissues. FGF signaling then induces migration of tracheal cells at the tip of each branch towards the Bnl signal, leading to the formation of a stereotypical set of primary branches in each tracheal metamere (Fig 1A; Sutherland et al. 1996). Outgrowth of the primary branches continues during following stages 13 and 14, leading to fusing of specific branches during stage 15 and formation of an interconnected network of tracheal tubes. Branch fusion establishes connections of the tracheal metameres along the anterior/posterior axis by fusion of dorsal trunk branches, and at the dorsal midline by fusion of dorsal branches (Caviglia and Luschnig 2014). Specialized fusion cells at the tips of these branches attach to mesodermal bridge cells and subsequently form intercellular adherens junctions with the opposing tip cells, followed by de novo tracheal lumen formation (Hayashi and Kondo 2018; Krause et al. 2006; Wolf et al. 2002).

The interconnected tracheal system of stage 16 embryos contains branches of different shapes, cell compositions and target organ specificities (Fig 1B). While the dorsal trunks are multicellular tubes, i.e. multiple cells engulf the dorsal trunk lumen at any given area, most parts of all other branches are unicellular tubes. In these unicellular tubes, the tracheal lumen is surrounded by cells that form autocellular junctions (Kerman et al. 2006). Precise targeting and shaping of the different tracheal branches is achieved by various signals from the surrounding tissues that modify the genetic programs of tracheal cells. The transforming growth factor-B (TGF-B) ligand Decapentaplegic (Dpp) is expressed in ventral non-tracheal tissues and the dorsal leading edge of the epidermis and plays a central role in shaping the tracheal network. Dpp activates signaling by the TGF-B receptor Thickveins, thereby inducing expression of the transcription factor Knirps (Kni) in dorsal, visceral and ganglionic branches and the lateral trunk (Ribeiro et al. 2004). Kni activates a genetic program that leads to intercalation of tracheal cells, resulting in the formation of unicellular tubes (Fig 1C; Ribeiro et al. 2004; Chen et al. 1998). During primary branch migration, high levels of FGF signaling are restricted to the tip cells by lateral inhibition via Delta/Notch signaling (Zelzer and Shilo 2000). Upon reaching their target tissues, FGF signaling is inhibited in the primary tip cells and they are transformed to fusion cells or their migration is 

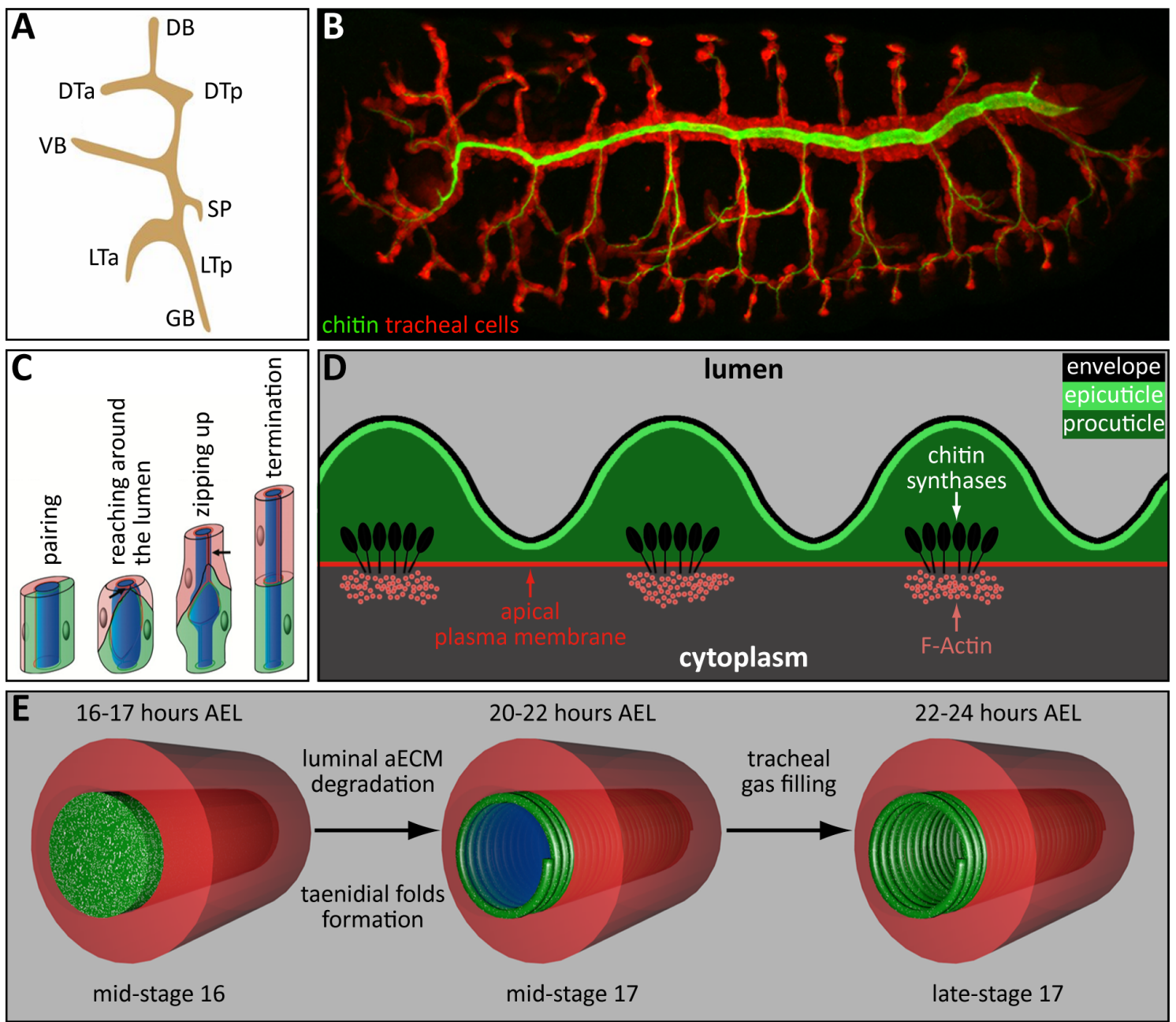

\section{Figure 1: The embryonic tracheal system of Drosophila melanogaster}

(A) A tracheal metamere in stage 12 contains stereotypical primary branches: the dorsal branch (DB), dorsal trunk anterior (DTa), dorsal trunk posterior (DTp), visceral branch (VB), lateral trunk anterior (LTa), lateral trunk posterior (Ltp), ganglionic branch (GB) and spiracular branch (SP). This panel was adapted from Hayashi and Kondo (2018). (B) Confocal LSM image of a stage 16 1-eve-1-LacZ embryo stained with FITC-labeld chitinbinding probe (CBP; chitin, green) and anti-B-Gal antibodies (red). The B-Gal expression labels the tracheal cells. An intraluminal chitin cable is present throughout the whole tracheal system. This image was kindly provided by Ralf Pflanz, Max Planck Institute for Biophysical Chemistry, Göttingen, Germany. (C) Intercalation process model representing the four key steps of autocellular adherens junction formation. The adherens junctions (red and green lines), the cell bodies of two cells (red and green) and the tracheal lumen (blue) are shown. Initially paired cells intercalate by reaching around the lumen to form autocellular adherens junctions (arrows) which are then zipped up. This panel was adapted from Ribeiro et al. (2004). (D) Schematic cross-sectional view of the mature tracheal aECM taenidial folds. The tracheal aECM is a stratified structure and contains an outermost hydrophobic envelope layer (black), a protein-rich epicuticle layer (light green) and a chitinrich procuticle layer (dark green). Accumulations of chitin synthases (black ovals) and cytoplasmic actin fibers (red dots) are present at the base of the taenidia. The apical plasma membrane is shown in red. (E) Schema of tracheal aECM maturation and liquid clearance. The aECM (green) in the tracheal lumen is degraded after mid-stage 16 and the mature 
taenidial folds (green spiral) form at the apical side of tracheal cells (red) during mid-stage 17. Liquid (blue) is cleared from the tracheal lumen during late-stage 17. Time data refer to embryonic development at $22^{\circ} \mathrm{C}$.

halted (Hayashi and Kondo 2018; Miao and Hayashi 2016). Suppression of FGF signaling in the primary tip cells gives rise to FGF responsive cells in other parts of the branches that form the secondary tip cells. Secondary branching occurs simultaneously to terminal branching and both processes are again dependent on the Bnl/Btl FGF signaling pathway (Hayashi and Kondo 2018; Jarecki et al. 1999). Terminal branches are formed by the terminal cells that differentiate from tip cells when branches reach their target tissues. These terminal branches form intracellular tubules that are guided by local oxygen demand and differ from the multicellular tubes of dorsal trunks and unicellular branches (Centanin et al. 2008; Kerman et al. 2006).

While the genetic control of $D$. melanogaster tracheal system development is one of the most well-understood examples in organogenesis, many downstream mechanisms that contribute to growth and shaping of the tracheal system are unknown. However, the formation and remodeling of transient aECM structures during embryonic tracheal development emerged as central regulators in shaping and size-control of the tracheal system.

\subsubsection{The role of transient apical extracellular matrix during tracheal development}

The formation of elaborate aECM structures plays essential roles in function and morphogenesis of animal organs. These aECMs protect organs from infection, dehydration and chemical or physical damage and provide scaffolds and mechanical cues during organogenesis. In insects, the mature aECM (cuticle) serves as an exoskeleton and is composed of chitin, proteins, lipids and small organic molecules. The mature cuticle of insects is formed by epithelia of the epidermis, trachea, hindgut and foregut and is essential for functions of these organs (Moussian 2010). Besides formation of a mature cuticle, transient aECM structures are formed in the $D$. melanogastertracheal system during embryogenesis. Research during the last decades revealed numerous functions of 
transient aECM structures in the developing tracheal system (reviewed in ÖztürkÇolak et al. 2016a).

In stage 13 of embryogenesis, the polarized tracheal epithelial cells start to secrete aECM components into the forming tracheal lumen. A major component of this intraluminal aECM is chitin, a beta $1,4-\mathrm{N}$-Acetlyglucosamine polymer which is synthesized by the transmembrane chitin synthase Krotzkopf verkehrt (Kkv) in the tracheal system (Moussian et al. 2005). By stage 16 of embryonic development, an intraluminal chitin cable is present throughout the whole tracheal system (Fig 1B). Synthesis and luminal deposition of chitin is essential for uniform expansion of the tracheal lumen and affects apical organization of the cytoskeleton in tracheal cells (Tonning et al. 2005). In addition to the requirement of chitin synthesis by Kkv, chitin modifications and its organization into parallel polymers is equally important for tracheal system development. Numerous factors have been implicated in these processes. The two chitin-modifying proteins Knickkopf (Knk) and Retroactive (Rtv) are essential for filamentous chitin organization (Moussian et al. 2006b). Mutants of knk or rtv show a similar phenotype of non-uniform tracheal tube expansion as $k k v$ mutant embryos that lack chitin synthesis, showing that organization of the tracheal intraluminal chitin cable into parallel polymers is essential for its function (Öztürk-Çolak et al. 2016a). Furthermore, chitin modifications by the secreted chitin deacetylases Serpentine (Serp) and Vermiform (Verm) are essential for control of tracheal lumen dilation (Luschnig et al. 2006). Lack of Serp or Verm causes excessive elongation of the tracheal dorsal trunk, presumably due to aberrant mechanical properties of the intraluminal chitin cable. The two chitin-binding proteins Obstructor-A (Obst-A) and Gasp also contribute to tracheal tube dilation, but their effect on the intraluminal chitin structure is currently unknown (Tiklová et al. 2013).

Precise control of chitin deposition and modifications in a spatially and temporal manner is essential for tracheal organogenesis. The initiation of chitin synthesis is regulated by the two Smad-like proteins Expansion (Exp) and Rebuf (Reb; Moussian et al. 2015). Expression of exp, reb and $k k v$ is inhibited by the zinc-finger transcription repressor Blimp-1 after sufficient chitin synthesis (Öztürk-Çolak et al. 2018). Recently, Reb was also identified as a regulator of temporal apical secretion of the chitin-binding protein Obst-A (Chandran et al. 2018). Besides these 
regulatory mechanisms, chitin deposition and apical secretion of chitin modifiers into the tracheal lumen depend on components of the canonical secretory pathway (Öztürk-Çolak et al. 2016A; Tsarouhas et al. 2007). In addition, translocation of the chitin deacetylases Serp and Verm to the tracheal lumen and apical localization of the chitin modifier $\mathrm{Knk}$ are regulated by the baso-lateral septate junction complexes (Nelson et al. 2010; Moussian et al. 2006b; Luschnig et al. 2006). While underlying molecular mechanisms are unknown, this process links establishment of the transepithelial tracheal barrier that is provided by the septate junctions with aECM formation and tracheal tube dilation. In summary, spatially and temporally controlled deposition of a transient intraluminal chitin cable and its modifications establish uniform growth of the tracheal lumen during stages 13 to 16, regulating tube size at the longitudinal anterior/posterior axis and the axial luminal diameter.

In addition to chitin and chitin modifying proteins, zona pellucida domain (ZPD) proteins are structural components of the transient intraluminal aECM cable. ZPD proteins were first identified in the eponymous Zona Pellucida ECM of mammalian oocytes and are present in virtually all animals (Gupta 2015; Jovine et al. 2005). While ZPD proteins contain a highly variable diversity of additional protein domains, they commonly localize to the ECM or are associated to cellular membranes (Jovine et al. 2005). The ZPD serves as a polymerization module, enabling the formation of macromolecular homo- and heteropolymeric elastic fibers of ZPD proteins (Jovine et al. 2002). Three ZPD proteins are known to be components of the tracheal aECM in the D. melanogaster embryo: Dumpy (Dpy), Piopio (Pio) and Trynity (Tyn). The dpy gene spans over $100 \mathrm{~kb}$ and encodes the largest known protein of $D$. melanogaster with a mass of 2,5 MDa (Gramates et al. 2017). At least 16 different protein isoforms are encoded by dpy that all contain a ZPD and predicted transmembrane domain at their carboxy-terminus and a varying number of up to 308 EGF-like domains (Gramates et al. 2017; Wilkin et al. 2000). In contrast, pio and tyn encode proteins with predicted mass of $51 \mathrm{kDa}$ and $81 \mathrm{kDa}$, respectively, that both contain ZPDs and predicted transmembrane domains (Gramates et al. 2017).

Pio and Dpy are crucial for tracheal network integrity. In the absence of either Pio or Dpy the intercalation of tracheal cells fails, leading to a disrupted tracheal network and the formation of multicellular cysts instead of unicellular branches 
(Bökel et al. 2005; Jazwinska et al. 2003). It was therefore suggested, that Pio and Dpy regulate termination of the intercalation process by stopping tracheal cell movements upon establishment of unicellular junctions (Fig 1C; Ribeiro et al. 2004). This function might be dependent on cell - aECM adhesion that is generated by Pio and Dpy since both proteins are also essential for attachment of epidermal apodemes to the cuticle (Bökel et al. 2005; Wilkin et al. 2000). Notably, functions of Pio and Dpy are most likely redundant, since pio, dpy double mutant embryos show the same phenotype as pio or dpymutant embryos (Jazwinska et al. 2003). Fluorescence recovery after photobleaching (FRAP) experiments showed, that Dpy fibrils in the tracheal lumen are an elastic cable that stretches during growth of the tracheal system (Dong et al. 2014). Its mechanical properties are dependent on extensive O-linked-N-acetylglucosamine modifications of Dpy (Sakaidani et al. 2011). These findings imply, that the intraluminal Dpy polymer is cross linked to other aECM cable components like chitin and connected to the tracheal cells that apply mechanical force to the Dpy cable by apical membrane expansion. Indeed, excessive apical membrane expansion by constitutive activation of the tyrosine kinase Src42A causes elongated tracheal tubes and increased stretching of the intraluminal Dpy cable (Dong et al. 2014; Förster and Luschnig 2012). Pio was suggested to provide the link between tracheal cells and the aECM due to its transmembrane domain and putative ability to polymerize with other ZPD proteins (Öztürk-Çolak et al. 2016a; Dong and Hayashi 2015). In contrast to Pio and Dpy, Tyn is not essential for tracheal network integrity. While Tyn is an integral component of the transient intraluminal aECM cable, recent studies suggest that it only plays a role during formation of the mature aECM in later developmental stages, which will be discussed in the following chapter.

\subsubsection{Tracheal cuticle formation and liquid clearance}

The intraluminal tracheal aECM cable is a transient structure and its degradation starts in late-stage 16. Simultaneously, the mature tracheal aECM cuticle is formed (Öztürk-Çolak et al. 2016b). The tracheal cuticle forms a unique structure of helical protrusions - the so-called taenidial folds - that run perpendicular to the tracheal tube axis (Fig 1D-E; Wigglesworth 1990). It is thought, that this patterning prevents the tracheal tubes from collapsing while also allowing 
contraction and expansion, similar to the hose of a vacuum cleaner. Despite its specialized structure, the mature tracheal aECM shows a similar stratified organization as the epidermal cuticle, comprising an outermost hydrophobic envelope layer that contains lipids and unknown proteins, a thin subjacent epicuticle layer that contains unknown proteins, and a procuticle layer that lines the apical plasma membrane and mainly contains chitin (Fig 1D; Moussian 2010; Moussian et al. 2006a). The size of the helical taenidia depends on the size of the corresponding tracheal branch, while spacing between the taenidia is relatively constant throughout the tracheal system (Hannezo et al. 2015).

The mature tracheal aECM is a supracellular structure and taenidial folds patterning extends throughout the whole tracheal system, unaffected by cell boundaries (Fig 1E). Remarkably, taenidial folds formation is nevertheless dependent on the formation of sub-apical F-actin rings and organization of the cortical cytoskeleton, respectively (Fig 1D; Öztürk-Çolak et al. 2016a; Uv and Moussian 2010; Matusek et al. 2006). Recent studies implicate that organization of the cortical F-actin rings is driven by a self-organizing mechanism and primes the formation of taenidial folds (Hannezo et al. 2015). However, taenidial folds patterning and F-actin-ring organization seem to be mutually dependent since chitin deposition in the procuticle layer is a prerequisite for cortical F-actin organization. This mechanism might be regulated by a feedback loop in which the positioning of chitin synthases at the taenidial bases (Fig 1D) and resulting chitin synthesis inhibits the activity of Src42A and thereby stabilizes underlying F-actin rings (Öztürk-Çolak et al. 2016b).

Chitin synthesis is essential for formation of the mature tracheal aECM, as it is a prerequisite for $D$. melanogaster cuticle formation. Similarly, chitin-modifying and chitin-binding proteins and their transcriptional regulators that were mentioned in chapter 1.1.2 contribute to shaping the tracheal taenidial folds. Mutations in genes encoding for these proteins cause deformed taenidia without affecting helical patterning of the mature tracheal aECM (Öztürk-Çolak et al. 2016a). It was also reported that shaping of the mature tracheal aECM is dependent on the ZPD protein Dpy and the apical transmembrane protein Uninflatable (Uif; Zhang and Ward 2009; Wilkin et al. 2000). However, mutant phenotypes remain to be analyzed in detail and underlying mechanisms are unknown. Since mature 
tracheal aECM formation is dependent on a secretory wave of aECM materials, many components of the secretory pathway were identified to be essential for taenidial folds formation (Öztürk-Çolak et al. 2016a; Armbruster and Luschnig 2012). Recently, the ZPD protein Tyn was shown to affect nanopatterning of the mature tracheal aECM (Itakura et al. 2018). While this role does not affect helical patterning, stratified structure and overall shape of the taenidial folds, it is essential for regulation of tracheal cuticle permeability to water and solute molecules. In addition, Tyn mediates the formation of valve-like structures at the posterior spiracles that might contribute to pressure regulation in the tracheal system (Wang et al. 2018).

After establishment of the tracheal cuticle and removal of the intraluminal aECM cable, remaining liquid in the tracheal lumen is exchanged with gas in a process called liquid clearance (LC) in late-stage 17 of embryogenesis (Fig 1E). This process is initiated by formation of a gas bubble in a dorsal trunk at a central tracheal metamere and proceeds by gradual gas filling of the dorsal trunks followed by the remaining branches in order of decreasing diameter. Luminal liquid is absorbed by surrounding tissue and the gas is presumably generated in embryonic tissues (Jaspers et al. 2014; Manning and Krasnow 1993). Correct formation of the mature tracheal aECM is a prerequisite for gas filling. Accordingly, lack of taenidial folds formation or lack of the hydrophobic envelope leads to a failure of tracheal LC (Jaspers et al. 2014; Moussian 2013). While mutants with deformed taenidial folds and tyn mutants mostly show initial gas filling of the tracheal system, the tubes refill with liquid during larval stages and often display molting defects of the tracheal cuticle (Itakura et al. 2018; Öztürk-Çolak et al. 2018; Wang et al. 2018; Öztürk-Çolak et al. 2016A; Moussian et al. 2015; Pesch et al. 2015). Formation of the transient intraluminal aECM as well as mature tracheal cuticle formation are dependent on exocytotic pulses. Vice versa, removal of the intraluminal aECM cable and intraluminal liquid is dependent on endocytotic pulses that are driven by the GTPase Rab5 and the conserved J-domain transmembrane protein Wurst among other factors (Behr et al. 2007; Tsarouhas et al. 2007). In parallel to the intake of liquid, embryonic tissues were suggested to generate a gas pressure that ultimately causes tracheal LC (Manning and Krasnow 1993). The transepithelial barrier that is provided by the septate junctions is essential for tracheal LC by regulating the paracellular flow of solute molecules (Izumi and Furuse 2014). 
Mutants that lack septate junction formation show no gas filling of the tracheal system (Hildebrandt et al. 2015; Hijazi et al. 2009; Nelson et al. 2010; Llimargas et al. 2004; Wu et al. 2004; Behr et al. 2003). Notably, septate junctions are the analogous structure of vertebrate tight junctions and both contain claudins in their core domains (Furuse and Tsukita 2006). While molecular mechanisms that initiate tracheal gas filling in D. melanogaster are currently unknown, the activity of epithelial sodium channels ( $\mathrm{ENaCs}$ ) encoded by the pickpocket (ppk) gene family was suggested to drive tracheal LC by mediating liquid absorption (Liu et al. 2003). This function may be equivalent to the well-established role of $\mathrm{ENaCs}$ in liquid clearance and homeostasis of mammalian airways (Matalon et al. 2015; Hummler and Planès 2010). However, the roles of D. melanogaster ENaCs in tracheal LC remain elusive, partly due to redundant functions of the 9 ppk genes expressed in the embryonic tracheal system (Liu et al. 2003).

Many processes and mechanisms of tracheal aECM formation, degradation and remodeling are currently unknown. With the exception of chitin synthesizing and modifying proteins, specific functions and interaction partners of aECM effectors remain to be identified. Most notably, mechanisms and factors that drive disassembly and degradation of the transient intraluminal aECM cable and initiate mature tracheal cuticle formation are still unknown. Recently, the transcription factor Ichor was shown to affect intraluminal aECM clearance and taenidial folds formation in larval terminal branches, presumably by activating expression of the serine protease Lumens interrupted (Lint; Rosa et al. 2018). However, further studies have to show whether these roles apply to the tracheal system during embryogenesis. Finally, the mechanisms of supracellular patterning of the mature tracheal aECM and initiation of liquid clearance are poorly understood. Promising candidates for regulation of aECM remodeling and degradation are proteolytic enzymes, as suggested by the role of Lint (Rosa et al. 2018). It is well established in vertebrate model systems, that differentiation of aECM structures and cell - aECM interactions are regulated by proteases during epithelial development (Bonnans et al. 2014; Antalis et al. 2011). One of the most well-known regulators of vertebrate aECM degradation and remodeling in epithelial development is the matriptase-prostasin proteolytic cascade, which will be discussed in the following chapter. 


\subsection{The matriptase-prostasin proteolytic cascade}

The matriptase-prostasin proteolytic cascade is a key regulator of epithelial development, homeostasis and formation of ECM structures in numerous vertebrate tissues (Bugge et al. 2007). Its deregulation is furthermore implicated in many pathological conditions including cancer, congenital diseases and viral infections (Beaulieu et al. 2013; Miller and List 2013). The central component of this proteolytic cascade is matriptase, regulating a complex downstream network of proteases, membrane-bound receptors, cell-junction components and ECM proteins (Miller and List 2013; Bugge et al. 2007). Since the first studies on matriptase about two decades ago, many functional mechanisms and substrates of matriptase, including its essential interaction with prostasin, have been unraveled (Lin et al. 1999). Despite this extensive research, understanding of downstream pathways, proteolytic substrates and regulatory mechanisms of the matriptase-prostasin proteolytic cascade is incomplete. Dissection of the involved complex interaction networks remains challenging, due to tissue and pathogenic condition specific differences in matriptase and prostasin functions (Miller and List 2013).

\subsubsection{Regulation and function of the matriptase-prostasin proteolytic cascade during vertebrate development}

Matriptase is a member of the type II transmembrane serine protease (TTSP) protein family, which is defined by a specific protein domain organization. TTSPs contain a short amino-terminal cytoplasmic tail, followed by a transmembrane domain and an extracellular carboxy-terminus (Hooper et al. 2001). The extracellular part is comprised of a stem region that varies in length and can contain diverse protein domains, and a carboxy-terminal serine protease domain with a highly conserved chymotrypsin (S1) fold. Both, matriptase and prostasin are membrane-anchored serine proteases. While matriptase contains a transmembrane domain, prostasin is associated to membranes via a carboxy-terminal glycophosphatidylinositol (GPI) -anchor (Aggarwal et al. 2013). Like all serine proteases, matriptase and prostasin are translated as single-chain zymogens and activated by proteolytic cleavage at a conserved site in the amino-terminus of the catalytic domain (Miller and List 2013; Polgár 2005). Following activation, the 
catalytic domains remain linked to the stem- or pro-domains by a conserved disulphide bridge (Bugge et al. 2007). It was shown that the prostasin zymogen is activated via cleavage by matriptase in vivo and in vitro (Netzel-Arnett et al. 2006). Reciprocally, prostasin regulates matriptase zymogen activation by a nonenzymatic mechanism, i.e. prostasin does not cleave the matriptase zymogen (Friis et al. 2013). Instead, cleavage of the matriptase activation site is mediated by the intrinsic catalytic activity of the matriptase zymogen, representing autocatalytic activation which is found in several TTSPs (Szabo and Bugge 2011). Recent studies suggest that the matriptase zymogen contains enough catalytic activity to execute most of the physiological functions of matriptase and that zymogen activation is only necessary to enhance its catalytic activity and stability (Friis et al. 2017; Tseng et al. 2017).

Initiation of the matriptase-prostasin proteolytic cascade depends on the catalytic activity of matriptase. Accordingly, autocatalytic zymogen activation and activity of matriptase is tightly regulated by a unique highly complex network of inhibitors and co-factors. Two essential regulators of matriptase activity are its cognate inhibitors hepatocyte growth factor activator inhibitor (HAI) -1 and HAI-2, which are also potent inhibitors of prostasin (Friis et al. 2014; Miller and List 2013; Oberst et al. 2005). Interestingly, dimerization of matriptase with its inhibitor HAI-1 is required for autocatalytic matriptase zymogen activation, in addition to regulation of matriptase zymogen activation by prostasin mentioned above (Lin et al. 2008). Besides its inhibitory function, HAI-2 regulates the secretory transport of matriptase and prostasin to cellular membranes and shedding of the matriptase ectodomain (Nonboe et al. 2017; Friis et al. 2014). Shedding of matriptase results in release of the stem region and attached catalytic domain from cellular membranes into the extracellular space and occurs in a tissue dependent manner (Bugge et al. 2007). Ectodomain shedding is mediated by self-cleavage of matriptase in its stem region which is promoted by prostasin and HAI-1 and inhibited by HAI-2 (Nonboe et al. 2017; Friis et al. 2014). However, HAI-2 is also required for translocation of matriptase to cellular membranes which is a prerequisite for matriptase ectodomain shedding (Friis et al. 2014). HAI-1, HAI-2, matriptase and prostasin are co-expressed in most tissues and regulation of the matriptase-prostasin proteolytic cascade seems to be achieved by differential 
expression levels and subcellular localization of these four components (Lee et al. 2018; Chang et al. 2015; Miller and List 2013).

While being widely expressed in vertebrate epithelial tissues, the roles of matriptase and prostasin in organogenesis were mainly analyzed in epithelia of the epidermis, intestine and placenta (Miller and List 2013; Antalis et al. 2011). Studies in mice showed that the matriptase-prostasin proteolytic cascade is essential for epithelial barrier function in the intestine and epidermis (Fig 2), and formation of the feto-maternal barrier in placental development (Szabo et al. 2014;

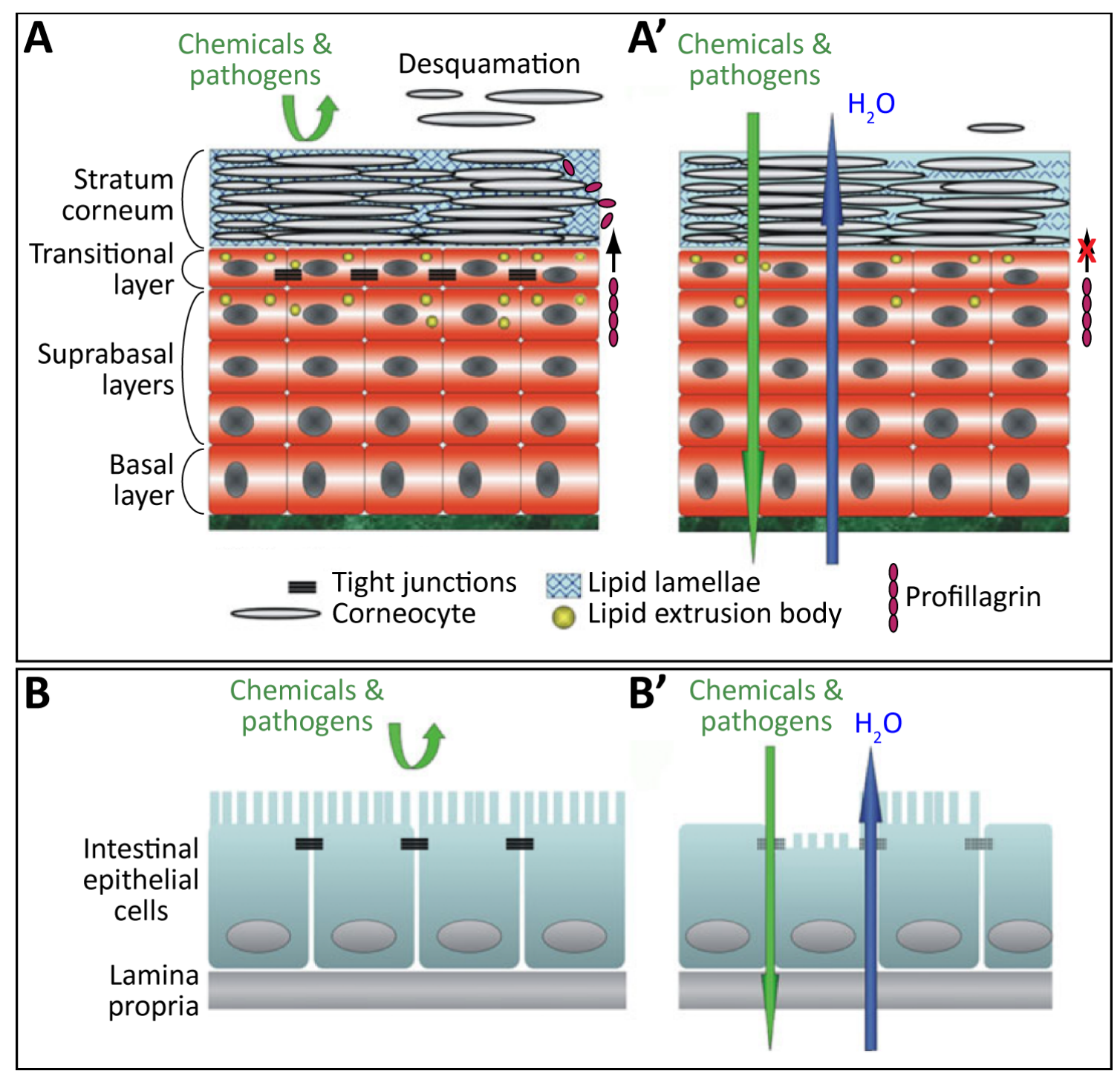

Figure 2: Roles of matriptase and prostasin in the epidermis and intestine of mice

Schemata of the mouse wild-type skin (A) and intestine (B) and matriptase or prostasin deficient skin (A') and intestine (B'). Defects in the skin include loss of tight junctions, loss of lipid extrusion, aberrant lipid lamellae formation, and lack of profillagrin processing resulting in impaired desquamation (A-A'). Defects in the intestine epithelium include loss of barrier function, loss of polarity, lack of mucin and deregulation of tight junction components claudin-2 and claudin-7. This figure was adapted from Miller and List (2013). 
Miller and List 2013). The vertebrate epithelial barrier function is provided by the tight junction protein complexes. Turn-over or expression levels of tight junction core components including claudins are regulated by matriptase and prostasin in the intestine, epidermis and placenta of mice (Wu et al. 2017; Szabo et al. 2014; Leyvraz et al. 2005). In the intestine, matriptase cleaves the epithelial cell adhesion molecule (EpCAM) which stabilizes the tight junction component claudin7, and thereby regulates turn-over of claudin-7 (Wu et al. 2017). However, a general mechanism by which the matriptase-prostasin proteolytic cascade regulates epithelial barrier function is currently unknown. Besides their role in tight junction formation, matriptase and prostasin are essential for stratum corneum formation, desquamation and hair follicle formation in the skin (Fig 2AA'; Miller and List 2013). The stratum corneum is the outermost layer of the skin and comprises dead cells (corneocytes) that are embedded in a lipid-rich aECM (Fig 2A-A'). Terminal differentiation of keratinocytes into corneocytes is driven by the matriptase-prostasin proteolytic cascade, partly by proteolytic processing of profillagrin (Ovaere et al. 2009; List et al. 2003). Cleavage of the large profillagrin polyprotein generates multiple fillagrin monomers that are integrated into the stratum corneum aECM and contribute to corneocyte differentiation (Netzel-Arnett et al. 2006; List et al. 2006). Both, stratum corneum formation and tight junction formation are crucial to prevent dehydration and entry of pathogens and toxins in the skin (Fig 2A-A'). In addition, prostasin regulates hydration of the skin and the influx of sodium ions by mediating the proteolytic activation of ENaCs (Carattino et al. 2014). The role of matriptase in epidermal development is not restricted to mammals, since matriptase also regulates integrity of the epidermal epithelial cell layer in zebrafish (Schepis et al. 2018; Carney et al. 2007). However, molecular downstream mechanisms of the matriptase-prostasin proteolytic cascade during epithelial development are not understood comprehensively and its function in various epithelial tissues remains elusive. To some extend, this is due to a focus of many studies on the role of matriptase and prostasin in the context of pathological conditions. 


\subsubsection{Role of the matriptase-prostasin proteolytic cascade in pathogenesis}

Many human pathological conditions were linked to the deregulation of matriptase and prostasin. Most notably, the matriptase-prostasin proteolytic cascade is implicated in cancer formation and progression, influenza virus infection and cystic fibrosis as well as in congenital disorders (Pawar et al. 2019; Beaulieu et al. 2013; Miller and List 2013; Myerburg et al. 2008). Mutations in the matriptase encoding gene suppression of tumorigenicity-14 (ST14) were found to cause congenital ichthyosis which manifests in lack of desquamation and profillagrin processing in the skin, resembling the epidermal phenotype of matriptase mutant mice (Fig 2A'; Alef et al. 2009; List et al. 2007). In the Netherton syndrom which also manifests in skin defects, matriptase was suggested to mediate stratum corneum detachment by aberrant activation of pro-kallikrein proteases (Sales et al. 2010). In both cases, activity of prostasin is also deregulated causing decreased activation of epidermal ENaCs. Conversely, excessive activation of ENaCs by prostasin is implicated in cystic fibrosis which is defined by sodium hyper-absorption in the airway epithelium (Myerburg et al 2008). Matriptase is also expressed in the epithelia of human airways and contributes to the activation of influenza virus particles during infection of the respiratory system (Beaulieu et al. 2013). It was shown that matriptase activates the hemagglutinin surface protein of several influenza subtypes by proteolytic cleavage and thereby promotes viral infection (Straus and Whittaker 2017; Beaulieu et al. 2013).

Besides the pathological conditions in the skin and airways mentioned above, matriptase and prostasin expression levels or proteolytic activities are associated with tumor formation and cancer progression in numerous tissues. Up-regulation of matriptase expression was observed in epithelial tumors of the breast, ovary, uterus, prostate, colon, cervix, and skin (Tanabe and List 2017). Tumorigenesis is promoted by the matriptase-prostasin proteolytic cascade via increased ECM degradation which allows invasion of tumor cells into surrounding tissues, and via activation of cell-surface receptors which activate pro-oncogenic signaling pathways (Tanabe and List 2017; Webb et al. 2011). Most notably, matriptase was shown to proteolytically activate the paracrine hepatocyte growth factor (HGF) and the cell surface proteinase-activated receptor 2 (PAR-2) during cancer progression (Pawar et al. 2019; Zoratti et al. 2015). Activated HGF promotes cell migration, proliferation and survival by activating its receptor c-Met, while PAR-2 influences 
inflammatory signaling pathways (Pawar et al. 2019; Yamasaki et al. 2018). Furthermore, matriptase activates macrophage stimulating protein 1, a growth factor that regulates cell survival and morphology through its receptor Recepteur d'Origine Nantais (Bhatt et al. 2007). Simultaneously, the matriptase-prostasin proteolytic cascade promotes activation of ECM degrading matrix metalloproteinases and is implicated in degradation of the ECM components nidogen-1, laminin and fibronectin (Ko et al. 2015; Jin et al. 2006; Satomi et al. 2001). The roles of matriptase, prostasin and their inhibitors HAI-1 and HAI-2 in pathogenic conditions are subject to extensive research and many underlying mechanisms have been uncovered. However, these molecular mechanisms were mostly not studied in the context of normal epithelial development. A better understanding of the physiological roles of matriptase and prostasin in activation of cell surface receptors and ECM degradation might strongly contribute to the understanding of their roles in diseases.

\subsection{Aims of this thesis}

In this thesis, I will analyze the roles of proteases in embryonic tracheal development of $D$. melanogaster. The primary focus of these analyses will lie on the role of serine proteases in formation, remodeling and degradation of the tracheal aECM as well as on their role in tracheal gas filling. Furthermore, I will investigate interactions between proteases in tracheal development to determine the organization of putative proteolytic cascades, since such cascades were identified as central regulators of vertebrate ECM differentiation. These analyses should provide insights into proteolytic mechanisms of tracheal aECM maturation and liquid clearance and broaden our understanding of proteases in animal development and disease. 


\section{Results}

Tracheal gas filling in late embryogenesis of $D$. melanogaster embryos is an indication for proper tracheal development. Accordingly, knock-downs or knockouts of genes that are essential for tracheal development often cause lack of tracheal gas filling. To identify novel genes that are essential for tracheal organogenesis, M. Jaspers carried out an RNA interference (RNAi) screening for genes that are required for tracheal LC (Jaspers 2011). Interestingly, three genes that were detected in this screening are predicted to encode for serine proteases. To confirm these findings, I performed trachea specific RNAi-mediated knock downs of the three serine protease encoding genes CG34350, CG8213 and CG4386 using the UAS/Gal4 system.
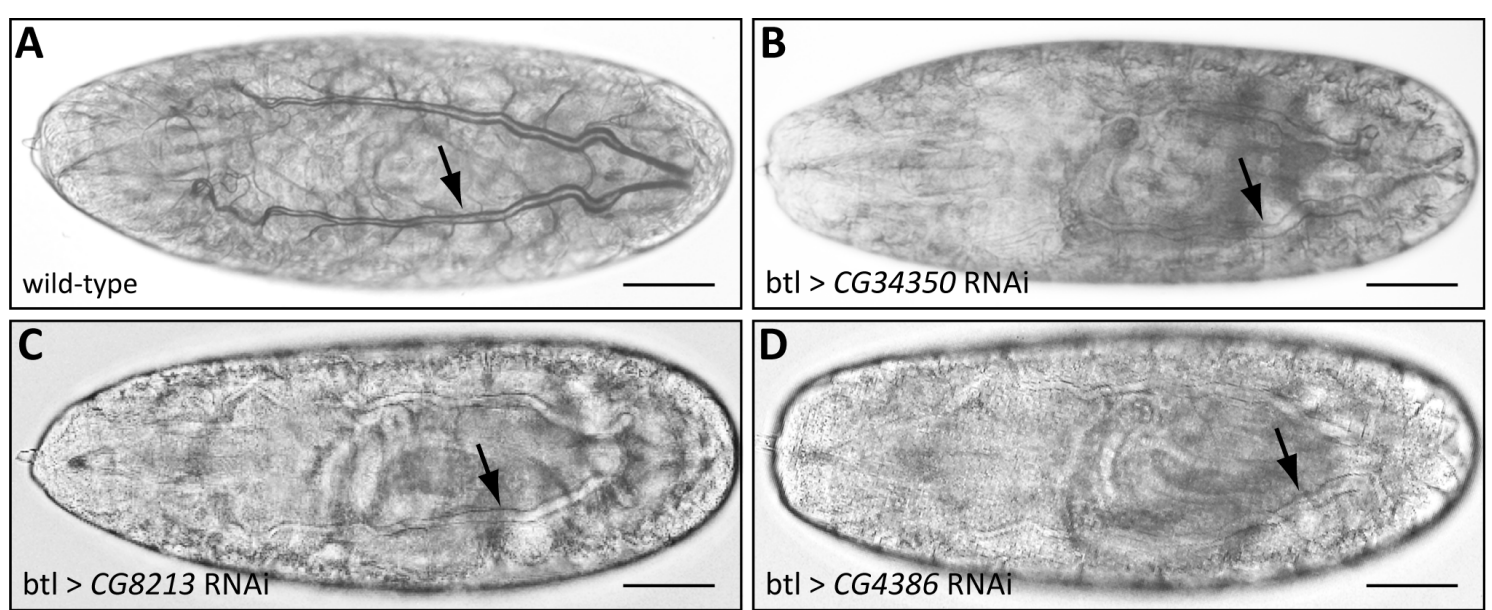

Figure 3: RNAi-mediated knockdowns of serine protease encoding genes cause lack of tracheal gas filling

Bright field light microscopic images of stage 17 wild-type (A), btl-Gal4; UAS-RNAiGD13443(B), btl-Gal4; UAS-RNAi-KK105169 (C) and btl-Gal4; UAS-RNAi-GD5850 (D) embryos. Wild-type embryos display a gas-filled tracheal system (arrow in A). RNAimediated tracheal knockdown of $C G 34350$ (B), CG8213 (C) or $C G 4386$ (D) leads to a lack of tracheal gas filling (arrows in B-D). Scale bars correspond to $50 \mu \mathrm{m}$.

Wild-type embryos undergo LC during embryonic stage 17, clearly visible by the strong contrast between the gas filled tubes and surrounding tissue (Fig 3A). Pantracheal expression of UAS-RNAi transgenes targeting CG34350 (UAS-RNAiGD13443), CG8213 (UAS-RNAi-KK105169) or CG4386 (UAS-RNAi-GD5850) led to 
lack of LC (Fig 3B-3D). Such embryos hatched but died during the first instar larval stage. Mesodermal (mef2-Gal4) or endodermal (Y48-Gal4) RNAi-mediated knockdown of these genes generated wild-type like, fertile flies. Thus, the RNAimediated LC phenotypes are tissue specific, indicating that CG34350, CG8213 and CG4386 are required for normal function of the tracheal system. CG34350 is also known as Notopleural $(\mathrm{Np})$ that was identified via a dominant mutation that manifests in the notopleural bristles of the adult fly (Bridges et al. 1936). In a recent study, CG8213 was suggested to play a role in processing of luminal tracheal matrix proteins in larval terminal branches and was named lumens interrupted (lint; Rosa et al. 2018). CG34350 and CG8213 will therefore be referred to as $N p$ and lint, respectively. In the following chapters, detailed functional analyses of $\mathrm{Np}$, lint and CG4386 during embryogenesis, and tracheal development in particular, will be carried out.

\subsection{Functional analysis of Notopleural during embryogenesis}

For a detailed analysis of the role of $N p$ during embryogenesis, genetic tools and established methods that are available for $D$. melanogaster were used. I generated loss-of-function alleles of $N p$ using CRISPR/Cas9 technology and $N p$ mutant embryos were then analyzed by immunohistochemical stainings, in vivo observations, transmission electron microscopy and immunoblotting. By these experiments, subcellular localization of aECM components and epithelial marker proteins, gas filling of the tracheal system, tracheal transepithelial barrier function and Np-mediated proteolysis were analyzed. To visualize localization of $\mathrm{Np}$ and putative Np interaction partners, CRISPR/Cas9 mediated homology directed repair was used to generate endogenously expressed fluorophore-labeled proteins. Tissuespecific rescue experiments using the UAS/Gal4 system were performed to determine whether $\mathrm{Np}$ has a non-cell autonomous function and whether human matriptase is functionally homologous to Np. Finally, cell-culture based experiments and biochemical assays with purified proteins were conducted to identify substrates and cleavage sites of $\mathrm{Np}$. In a first step, $\mathrm{Np}$ protein domain organization and sequence similarity to vertebrate proteins were analyzed in silico. 


\subsubsection{Notopleural encodes a type II transmembrane serine protease related to vertebrate matriptase}

Np encodes a single putative 1041 amino acids protein. In silico analysis predicts a type II transmembrane serine protease (TTSP) domain organization, which consists of a cytoplasmic amino-terminal tail, a transmembrane domain followed by an extracellular stem, and a carboxy-terminal trypsin-like serine protease catalytic domain (Fig 4A).

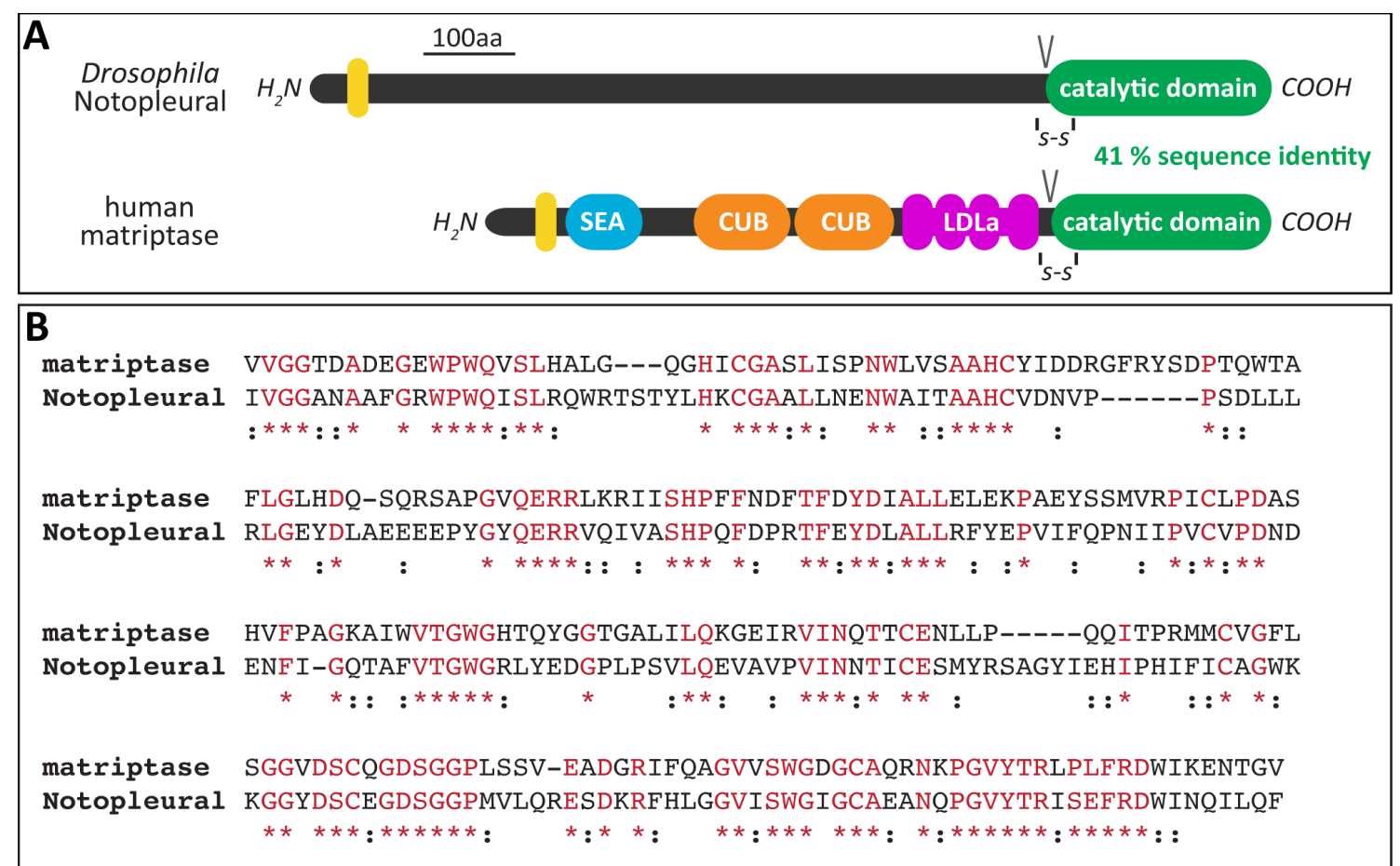

Figure 4: Notopleural encodes a type II transmembrane serine protease with sequence similarities to human matriptase

(A) Schemata of the protein domain organizations of D. melanogaster $\mathrm{Np}$ and human matriptase. The transmembrane domains (yellow), SEA (sea urchin sperm protein/enteropeptidase/aggrin), CUB (ㄷls/Clr, urchin embryonic growth factor, $\underline{b} o n e$ morphogenetic protein-1), LDLa (low-density lipoprotein receptor class $\underline{\mathrm{A}}$ ) and the catalytic serine protease domains (green) are shown. The conserved disulphide bridges (-S-S-) between catalytic domains and stem regions and the zymogen activation cleavage sites (V) are indicated. (B) Sequence alignment of the catalytic domains of D. melanogaster $\mathrm{Np}$ and human matriptase by Clustal omega (Sievers et al. 2011) reveals a $41 \%$ sequence identity (red; asterisks) and additional $20 \%$ sequence similarity (colon).

TTSPs are expressed as single-chain, catalytically inactive zymogens. Their catalytic domains are activated by proteolytic cleavage at a conserved motif between the stem regions and protease domains. Following cleavage, the catalytic 
domains remain linked to the stems via a conserved disulfide bond, resulting in activated two-chain TTSPs (Bugge et al. 2009; Hooper et al. 2001). A conserved cleavage site for activation ( $\mathrm{V}$ in Fig 4A) and two cysteines that may form a putative disulfide bond between the catalytic domain and the stem region are also found in Np (Fig 4A). When compared to human proteins using BLASTP (Altschul et al. 1997), $\mathrm{Np}$ shows the highest degree of sequence similarity to the TTSP matriptase. They share a sequence identity of $41 \%$ within their catalytic domains, while the extracellular stems and the cytoplasmic domains of both proteins show only minor sequence similarities (Fig 4). Furthermore, when comparing human matriptase to D. melanogaster proteins using BLASTP, Np shows the highest degree of sequence similarity. The ST14 gene that encodes matriptase is highly conserved in vertebrates (List et al. 2006). Accordingly, D. melanogaster $\mathrm{Np}$ shows the highest sequence similarity to matriptase homologs from Macaca mullatus, Mus musculus, Rattus norvegicus, Bos taurus and Danio rerio in BLASTP queries for proteins of these species. These observations imply that $N p$ encodes a putative TTSP with sequence similarities to the vertebrate TTSP matriptase.

\subsubsection{Notopleural is essential for tracheal gas filling}

For a detailed functional analysis of $N p$, I generated loss-of-function alleles using the CRISPR/Cas9 system (Fig 5A-5C). The obtained $N p^{P 6}$ and $N p^{C 2}$ alleles cause frame-shifts in the ORF followed by premature translation stops (Fig 5B-C). Furthermore, the $N p^{P 6}$ and $N p^{C 2}$ alleles fail to complement each other as well as Df(2R)BSC271, which deletes the chromosomal region 44F12-45A12 including $N p$. Embryos that are homozygous for the $N p^{P 6}$ or $N p^{C 2}$ alleles or transheterozygous for $N p^{P 6} / N p^{C 2}$ display lack of tracheal gas filling (Fig 5D). While $N p$ mutant embryos pass through all embryonic stages and show movements during embryonic stage 17, they are unable to hatch and die at the end of embryogenesis. To test whether the observed tracheal LC phenotypes were indeed caused by the CRISPR/Cas9 mediated mutations in $N p$, tracheal system specific rescue experiments were performed. An UAS- $N p$ responder line was established and used for btl-Gal4 driven pan-tracheal expression of $\mathrm{Np}$ in mutant embryos. $\mathrm{Np}$ mutant embryos with ectopic tracheal $N p$ expression from the UAS transgene show wild-type-like LC of the tracheal system (Fig 5E). However, these embryos still do not hatch as larvae, 
indicating essential functions of $N p$ in other tissues, apart from the tracheal system. The rescue experiments show, that the observed tracheal LC phenotype in $N p$ mutant embryos is due to loss of $N p$ expression and was not caused by offtarget effects during CRISPR/Cas9-mediated mutagenesis. These results indicate that $N p$ is essential for gas filling of the tracheal system.

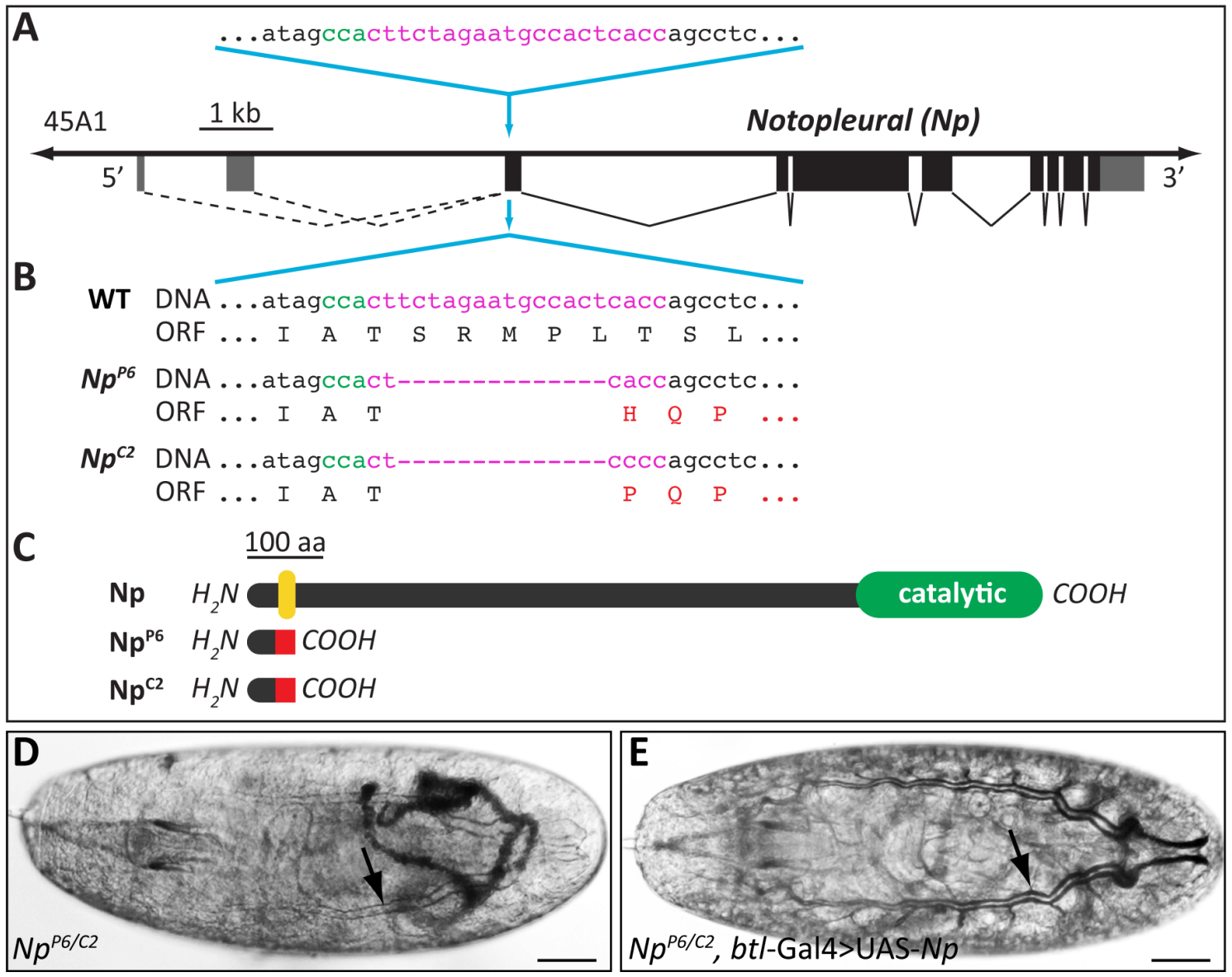

Figure 5: Generation of Notopleural loss-of-function alleles by CRISPR/Cas9-mediated mutagenesis

(A-C) Schema of CRISPR/Cas9-mediated mutagenesis to generate frame shift mutations in the 5' region of the $N p$ ORF. (A) Physical map of $N p$ genomic region shows the single guide RNA (sgRNA) recognition site (magenta letters) with PAM (green letters), translated DNA (black boxes) and UTRs (grey boxes). (B) Generated DNA indels cause ORF frame shifts in $N p^{P G}$ and $N p^{C 2}$ mutant alleles. (C) Frame shifts result in expression of truncated $\mathrm{Np}$ proteins. Transmembrane domain (yellow), catalytic serine protease domain (green) and regions with frame-shifted amino acid sequences (red) are indicated. (D-E) Bright field light microscopic images of stage $17 N p^{P 6} / N p^{C 2}$ (D) and $N p^{P 6}$,btl-Gal4 $/ N p^{C 2}$;UAS- $N p$ (E) mutant embryos. While $N p$ mutant embryos show no tracheal LC (arrow in D), $N p$ mutant embryos with tracheal expression of $N p$ display gas filling of the tracheal system (arrow in E). Scale bars correspond to $50 \mu \mathrm{m}$. 


\subsubsection{Notopleural is expressed in ectodermal tissues and localizes to apical plasma} membranes and the apical extracellular matrix

To visualize $N p$ transcript expression during embryogenesis, whole-mount RNA in situ hybridization stainings of wild-type embryos with a digoxigenin-labeled $N p$ antisense probe were performed. $N p$ transcripts are first detectable during stage 11 in cells of the tracheal placodes (Fig 6A). $N p$ expression persists in cells of the tracheal system and also appears in the salivary glands, the foregut, the hindgut, and the posterior spiracles in stages 13 to 15 (Fig 6B-D'). During stage $16, N p$ expression fades in the tracheal system, while epidermal expression increases from stage 15 to 16 (Fig 6D-E). Thus, $N p$ is differentially expressed in ectodermal tissues during embryogenesis in a developmental stage and organ specific manner.
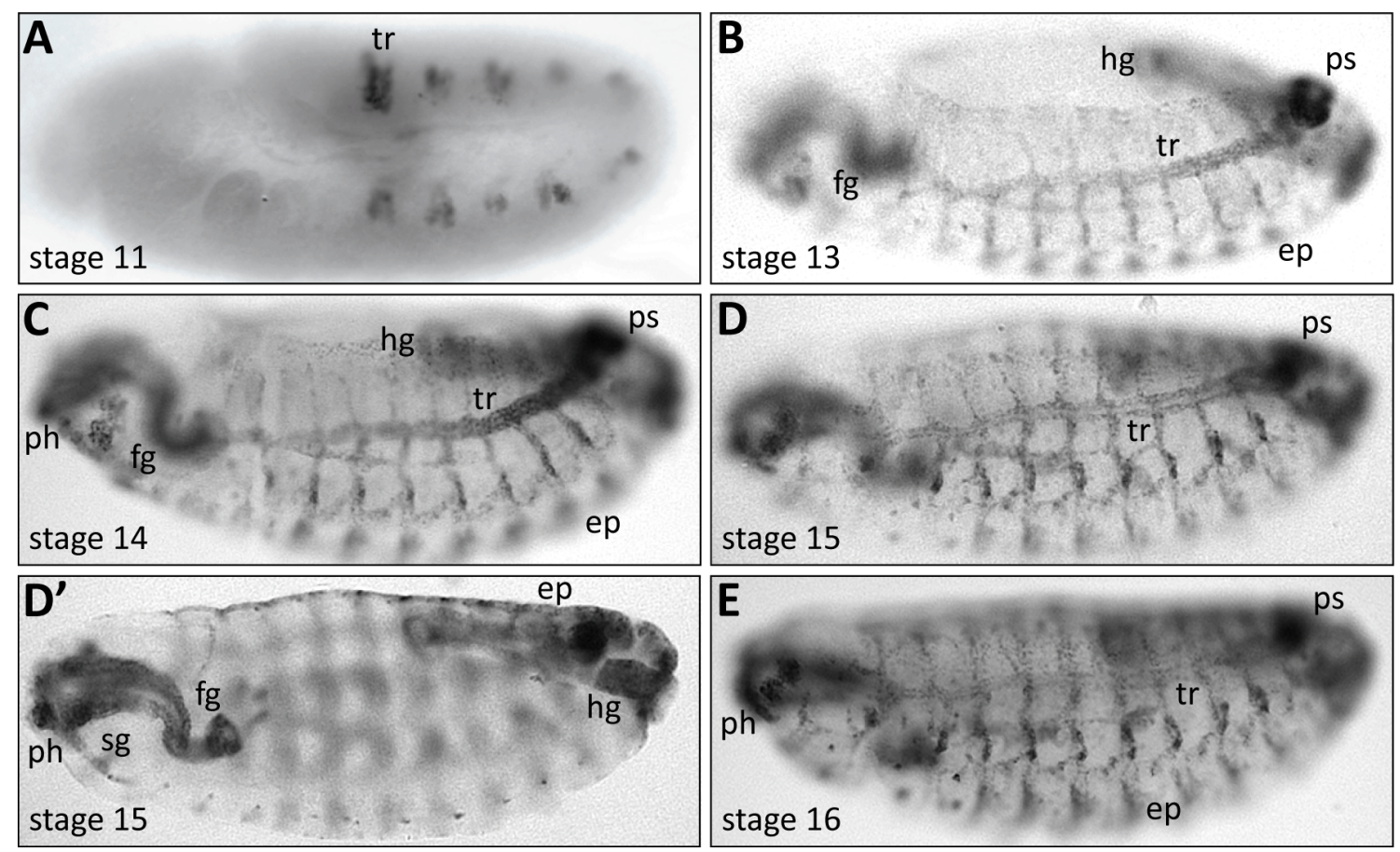

Figure 6: Notopleural is expressed in embryonic ectodermally derived tissues

Bright field light microscopic images of whole-mount RNA in situ hybridization stainings of wild-type embryos with a digoxigenin-labeled $N p$ antisense probe. $N p$ transcripts are first detectable in tracheal placodes (tr) during stage 11 (A). Expression of $N p$ is also detectable in the foregut (fg), hindgut (hg), epidermis (ep), posterior spiracles (ps), salivary glands (sg) and pharynx (ph) during embryogenesis. Tracheal $N p$ expression is most prominent during stages $14(\mathrm{C})$ and 15 (D, D' different focal planes) and fades in stage 16 (E).

To visualize $\mathrm{Np}$ protein localization, a $N p:$ GFP knock-in into the endogenous $N p$ locus was generated (Fig 7). In a first step, $N p$ exons coding for the catalytic domain and $N p 3^{\prime}$ UTR were exchanged with an attP site and a white selection 


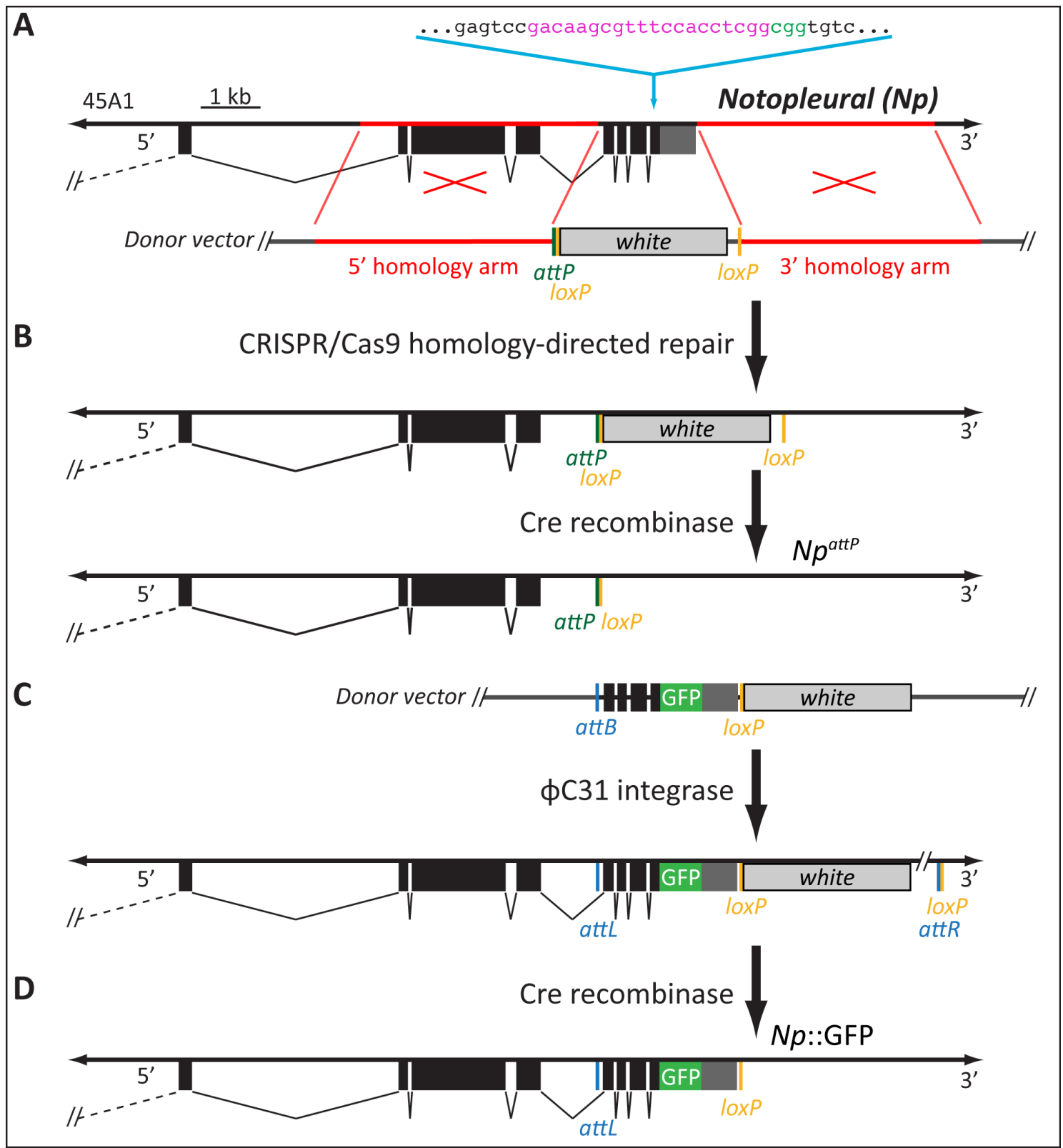

Figure 7: Generation of flies with endogenous expression of Notopleural::GFP using CRISPR/Cas9 technology.

(A) Schema of the $N p$ genomic locus together with a donor vector containing two homology arms (red), a white marker gene, an att $P$ site, and two lox $P$ sites. The sgRNA recognition site (magenta letters), PAM (green letters), translated DNA (black boxes) and UTRs (grey boxes) are indicated. (B) $N p$ locus after CRISPR/Cas9-mediated homology-directed repair (top) and Cre recombinase-mediated white gene excision (bottom), generating the $N p^{\text {att } P}$ allele. (C) Donor vector (top) for $\Phi$ C31-integrase-mediated knock-in of $N p$ genomic region with a GFP encoding sequence integrated 3' of the final protein coding exon, generating white $^{+}$flies with endogenous expression of GFP-tagged Np (bottom). (D) Np::GFP allele after Cre recombinase-mediated white gene excision. 
marker gene using CRISPR/Cas9 mediated homology-directed repair (Fig 7A-B). The obtained $N p^{\text {attP }}$ allele causes lethality and lack of tracheal gas filling when homozygously present, as previously reported for other $N p$ loss-of-function alleles (chapter 2.1.2). The $\Phi C 31$ integrase attP/attB system was then used to knock-in $N p$ genomic region with a GFP encoding sequence inserted 3 ' of the final protein encoding exon (Fig 7C-D). Np::GFP homozygous embryos show wild-type-like LC of the tracheal system and hatch as larvae. However, these larvae die in the end of third instar larval stage. To test whether lethality is caused by the attL and lox $P$ sites that remain in the $N p$ locus (Fig 7D), an attP/attB mediated knock-in was generated that only restores the wild-type $N p$ genomic region. This knock-in produces viable and fertile flies in homozygous conditions, indicating that lethality of $N p::$ GFP larvae is caused by the inserted GFP encoding sequence. Still, Np::GFP likely has normal function and localization in the embryo since $N p:$ GFP homozygous embryos and larvae are indistinguishable from wild-type, at least until third instar larval stage. Stainings of homozygous $N p:: G F P$ embryos with anti-GFP antibodies show that Np::GFP is detectable in all embryonic tissues that express $N p$ transcripts: the tracheal system, the salivary glands, the pharynx, the foregut, the hindgut, the posterior spiracles and the epidermis (Fig 8A-D). The spatial patterns of $N p$ transcripts and Np::GFP protein expression therefore coincide during embryogenesis. To analyze subcellular Np::GFP localization, stainings of $N p:: G F P$ embryos with anti-GFP and anti-Spectrin antibodies were performed. In the tracheal system, Np::GFP localizes to the intraluminal aECM during stages 16 and 17 (Fig 8E-F"; arrows in Fig 8E' and 8F'). In addition, Np::GFP is found in close association with the apical membrane of tracheal cells in stage 17 (Fig 8F-F"; arrowheads in Fig 8F'). In the hindgut (Fig 8G-G”) and the epidermis (Fig 8H-H”), Np::GFP is exclusively detectable at the apical cell membranes (arrowheads in Fig 8G' and 8H'). Whereas in the salivary glands, it localizes exclusively to the intraluminal aECM (Fig 8I-I"; arrow in Fig 8I').

These results show that Np localizes to the aECM and/or apical cell membranes in a tissue and developmental stage specific manner. Since Np contains a predicted transmembrane domain (Fig 4A), localization to the intraluminal extracellular space in the tracheal system and salivary glands suggests that the extracellular domain of $\mathrm{Np}$ is shedded from the membrane, as observed for vertebrate TTSPs including matriptase (Lin et al. 2008). 

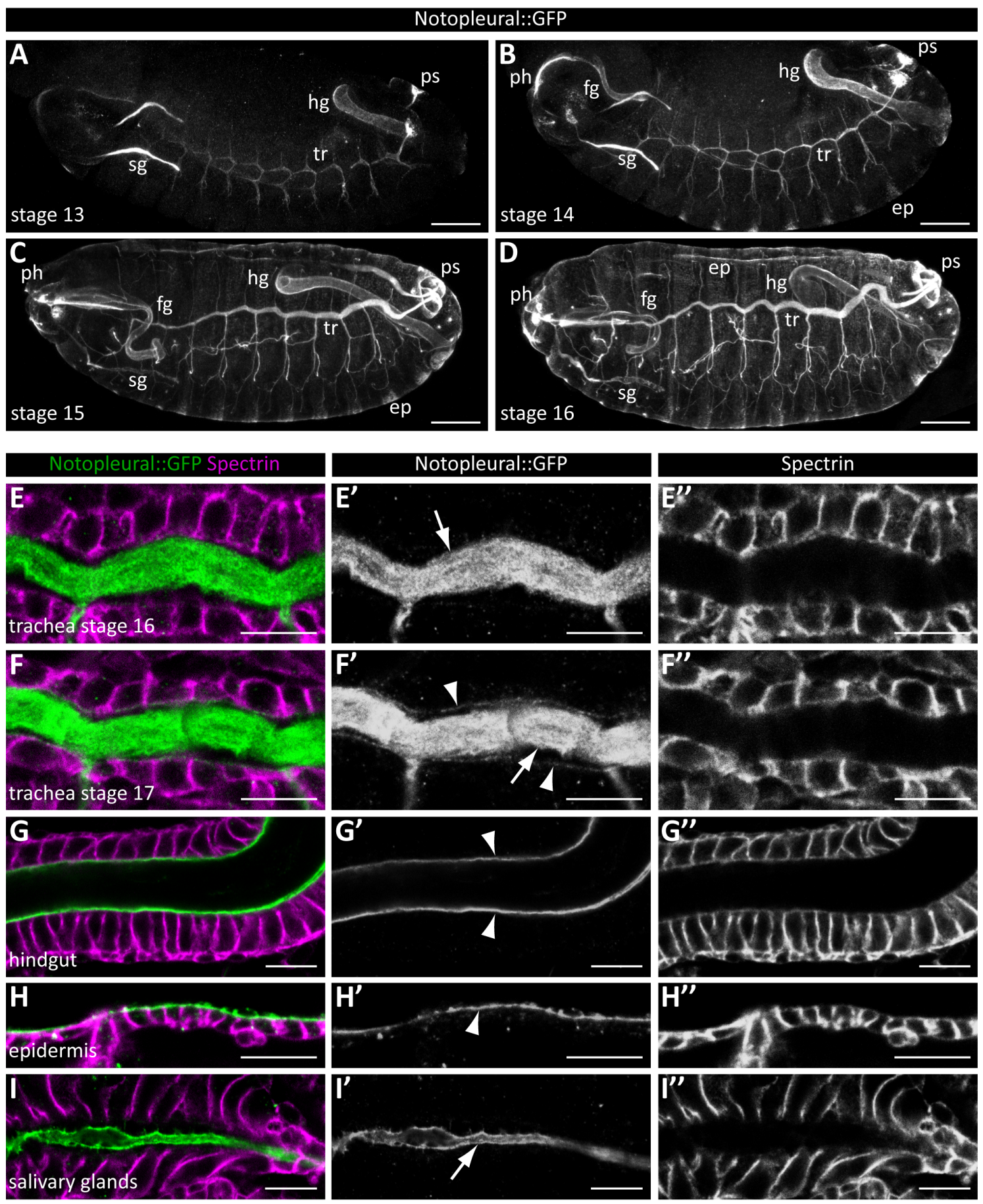

Figure 8: Notopleural localizes to apical plasma membranes and the apical extracellular matrix of embryonic ectodermal tissues

Confocal LSM images of whole-mount anti-GFP (A-D) or anti-GFP and anti-Spectrin (E-I") antibody stainings of Np::GFP embryos at stage 13 (A), 14 (B), 15 (C), 16 (D, E-E", G-I") and 17 (F-F"). (A-D) Np::GFP is detectable in the salivary glands (sg), foregut (fg), hindgut (hg), tracheal system (tr), posterior spiracles (ps),pharynx (ph) and epidermis (ep). Scale bars correspond to $50 \mu \mathrm{m}$. (E-I") Images of merged channels and single channels of Np::GFP (green) and Spectrin (magenta) stainings show Np::GFP localization in the trachea (E-F"), hindgut (G-G"), epidermis (H-H") and salivary glands (I-I"). Np::GFP localizes to the tracheal lumen during stages 16 and 17 (arrows in $\mathrm{E}^{\prime}$ and F') and to the apical membrane of tracheal cells during stage 17 (arrowheads in F'). In the hindgut and epidermis, Np::GFP 
localizes exclusively to the apical cellular membranes (arrowheads in $\mathrm{G}^{\prime}$ and $\mathrm{H}^{\prime}$ ). In the salivary glands, Np::GFP is detectable only in the intraluminal space (arrow in $J^{\prime}$ ). Scale bars correspond to $10 \mu \mathrm{m}$.

\subsubsection{Notopleural is essential for tracheal transepithelial barrier function but does not affect localization of septate junction components}

As observed in chapter 2.1.2, $N p$ mutant embryos lack gas filling of the tracheal system. Prerequisites for gas filling of the tracheal tubes are a continuous tracheal network without any fissures and a functional transepithelial barrier (Behr et al. 2003). Therefore, these features were analyzed in $N p$ mutant embryos. Tracheal network formation and integrity is wild-type-like in $N p$ mutant embryos, as shown by staining of the intraluminal chitin cable in stage 16 embryos (Fig 9A-B). To test the tracheal barrier function in $N p$ mutant embryos, Texas Red-labeled $10 \mathrm{kDa}$ dextran was injected into the haemocoel of stage 17 embryos (Lamb et al. 1998). Wild-type embryos show no diffusion of the dye into the lumen of the tracheal system (arrow in Fig 9C). In contrast, $10 \mathrm{kDa}$ dextran diffuses into the tracheal
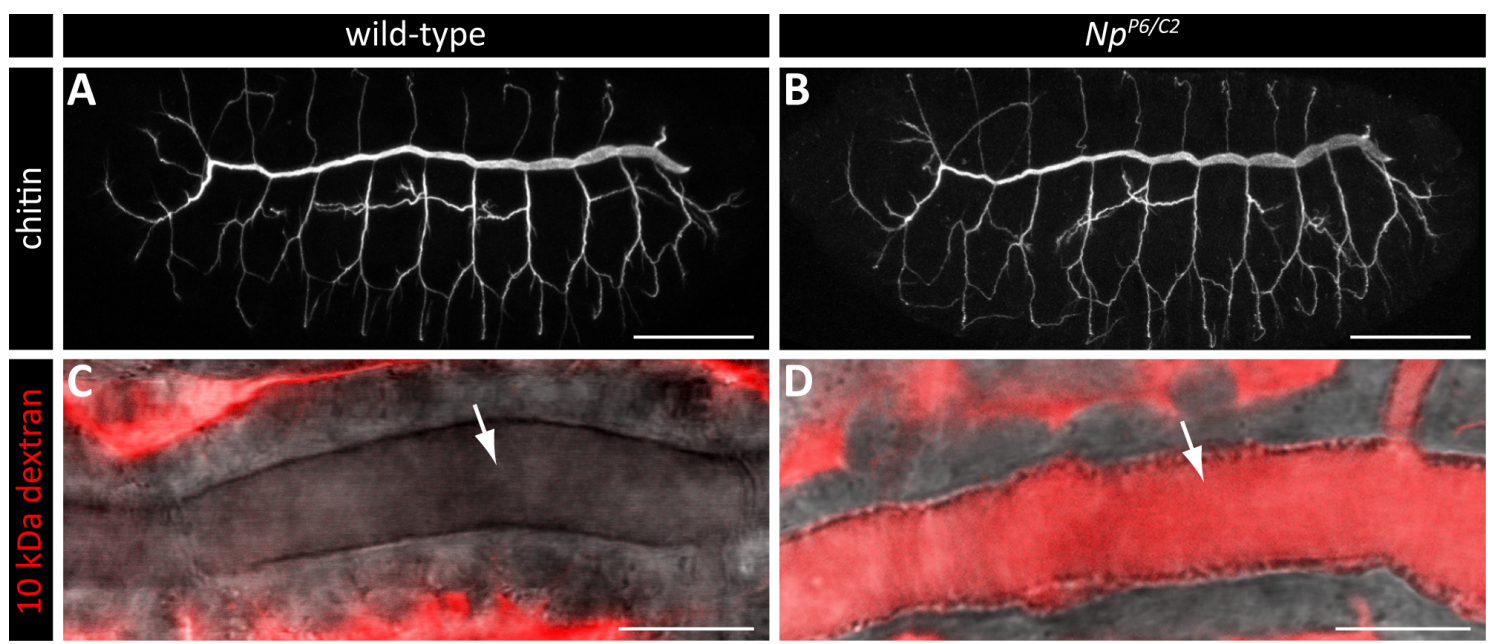

Figure 9: Notopleural is required for tracheal transepithelial barrier function

(A-B) Confocal LSM z-stack projection images of stage 16 wild-type (A) and $N p^{P 6} / N p^{C 2}$ mutant (B) embryos that were stained with FITC-labeled chitin-binding probe (CBP), visualizing tracheal intraluminal chitin. The tracheal network formation of $N p$ mutant embryos (B) is indistinguishable from wild-type embryos (A). Scale bars correspond to 50 $\mu \mathrm{m}$. (C-D) In vivo LSM confocal images of tracheal dorsal trunks of stage 17 wild-type (C) and $N p^{P 6} / \mathrm{Np}^{C 2}$ mutant (D) embryos after Texas Red-labeled $10 \mathrm{kDa}$ dextran injection into the haemocoel. Texas Red dextran (red) is not found in the dorsal trunk lumen of wild-type embryos (arrow in C), but is detectable in the dorsal trunk lumen of $N p$ mutant embryos (arrow in D). Scale bars correspond to $10 \mu \mathrm{m}$. 
lumen of $N p$ mutant embryos (arrow in Fig 9D), indicating a defective transepithelial barrier in the tracheal system.

Tracheal epithelial cells are polarized and form distinct apical and basal compartments and lateral cellular junctions (Tepass et al. 2001). The epithelial barrier function is mediated by the conserved baso-lateral multi-protein septate junction complexes that are fully established until stage 16 of embryonic development (Banerjee et al. 2006; Behr et al. 2003). To assess septate junction formation and epithelial polarization in $N p$ mutant embryos, whole-mount antibody stainings for several marker proteins in stage 16 embryos were performed. The septate junction components Kune-kune (Kune; Fig 10A-B"), Fasciclin3 (Fas3; Fig 10C-D") and Megatrachea (Mega; Fig 10E-F") localize wildtype-like in $N p$ mutant embryos. The adherens junctions component DE-cadherin (DE-cad) localizes to apico-lateral membranes of tracheal cells in both, wild-type and $N p$ mutant embryos (Fig 10A-B"). Uninflatable (Uif; Fig 10E-F") and Crumbs (Crb; Fig 10G-H") which are markers for the apical plasma membrane and apical cellular compartment, respectively, also show wild-type-like localization in $N p$ mutant embryos. However, $N p$ mutant embryos display abnormalities at the interface between tracheal apical plasma membranes and intraluminal space. In stage 16 wild-type embryos, the intraluminal chitin cable and apical plasma membrane are spatially separated by a gap in most areas and rarely show direct contacts (arrows in Fig 10E-E", G-G"). In contrast, the chitin cable continuously contacts the apical cellular surface in $N p$ mutant trachea (Fig 10F-F", H-H").

These results indicate that $\mathrm{Np}$ is essential for tracheal transepithelial barrier function while epithelial polarization and formation of septate junctions and adherens junctions in the trachea are not dependent on $\mathrm{Np}$. The observed $\mathrm{Np}$ mutant phenotype of chitin cable attachment to the tracheal cells suggests a role of $\mathrm{Np}$ in aECM formation and/or regulation of aECM - cell interactions. This role is consistent with $\mathrm{Np}$ localization at the tracheal apical plasma membranes and the aECM, shown in chapter 2.1.3. 


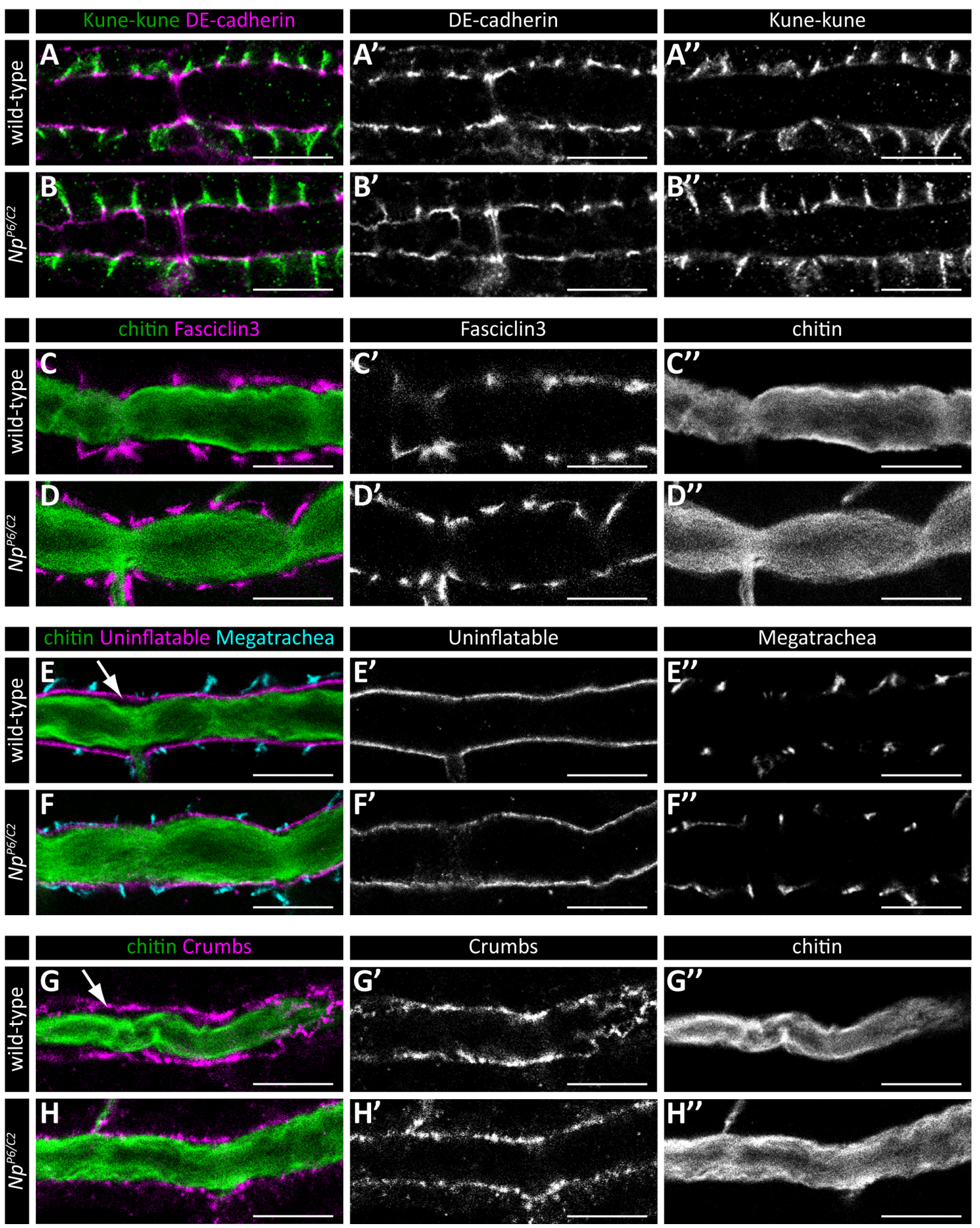

Figure 10: Localization of markers for septate junctions, adherens junctions and the apical compartment is not affected in Notopleural mutant embryos

Confocal LSM images of tracheal dorsal trunks of stage 16 wild-type (A-A", C-C", E-E", G$\left.\mathrm{G}^{\prime \prime}\right)$ and $N p^{P 6} / N p^{C 2}$ mutant (B-B", D-D", F-F"', H-H") embryos stained with anti-Kune and anti-DE-cad antibodies (A-B"), CBP (chitin) and anti-Fas3 antibodies (C-D"), CBP, anti-Uif and anti-Mega antibodies (E-F") or CBP and anti-Crb antibodies (G-H"). Localization of Kune, De-cad, Fas3, Uif, Mega and Crb is wild-type-like in $N p$ mutant trachea. In wild-type embryos, the intraluminal chitin cable is not in direct contact with the apical tracheal cell surface (arrows in E, G). In $N p$ mutant trachea, the luminal chitin cable is aberrantly attached to the apical cell surface (F, H). Scale bars correspond to $10 \mu \mathrm{m}$. 


\subsubsection{Tracheal epithelial barrier function maintenance is dependent on Notopleural}

The observation that $N p$ mutant embryos lack tracheal transepithelial barrier function but show normal localization of septate junction components was puzzling, since a defective barrier function is usually accompanied by disrupted septate junction morphology (Nelson et al. 2010). Therefore, the septate junction morphology in $N p$ mutant embryos was analyzed in more detail by transmission electron microscopy. Septate junctions are defined by a ladder-like arrangement of septae between baso-lateral cell membranes of adjacent cells. These structures are present in tracheal epithelia of wild-type and $N p$ mutant embryos (Fig 11). This observation indicates that structural aspects of septate junction morphogenesis are not dependent on $\mathrm{Np}$.
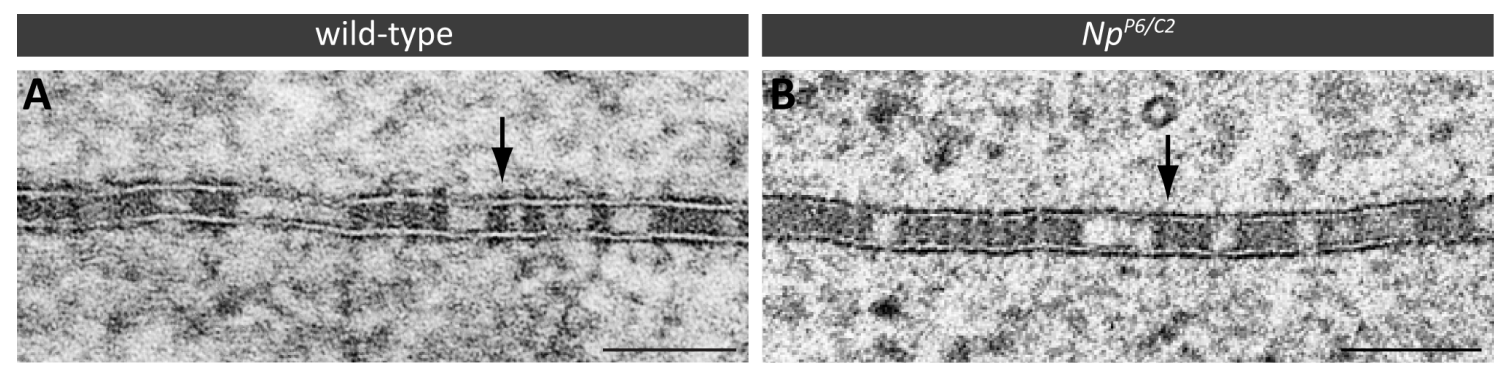

Figure 11: Septate junction morphology is wild-type-like in $\mathrm{Np}$ mutant embryos

Transmission electron micrographs of tracheal septate junctions in stage 17 wild-type (A) and $N p^{P 6} / N p^{C 2}$ mutant (B) embryos. Ladder-like septae (arrows) are present in tracheal epithelia of wild-type (A) and $N p$ mutant (B) embryos. Scale bars correspond to $0.1 \mu \mathrm{m}$.

All known mutants that display a defective embryonic transepithelial barrier function also show damaged morphology of the septate junctions ultrastructure and/or mis-localization of septate junction components (Hildebrandt et al. 2015; Nelson et al. 2010; Wu et al. 2004; Behr et al. 2003; Genova et al. 2003; Lamb et al. 1998). The data therefore suggest that $N p$ mutant embryos display a novel class of barrier function defects, in which the tracheal epithelial cell layer forms wild-typelike septate junctions complexes but lacks transepithelial barrier function. A well established septate junction core component is the claudin Mega. Embryos with loss-of-function mutations for mega lack septate junction formation and tracheal transepithelial barrier function (Behr et al. 2003). To compare the barrier function defects of $\mathrm{Np}$ mutant embryos with mega mutant embryos, $10 \mathrm{kDa}$ and $70 \mathrm{kDa}$ fluorophore-labeled dextran were injected into the haemocoels of control $\left(\mathrm{mega}^{\mathrm{VE896}} / \mathrm{mega}^{+}\right)$, mega $\mathrm{F}^{\mathrm{VE} 896} / \mathrm{mega}^{\mathrm{VEs} 96}$ mutant, and $N p^{P 6} / \mathrm{Np}^{C 2}$ mutant embryos 

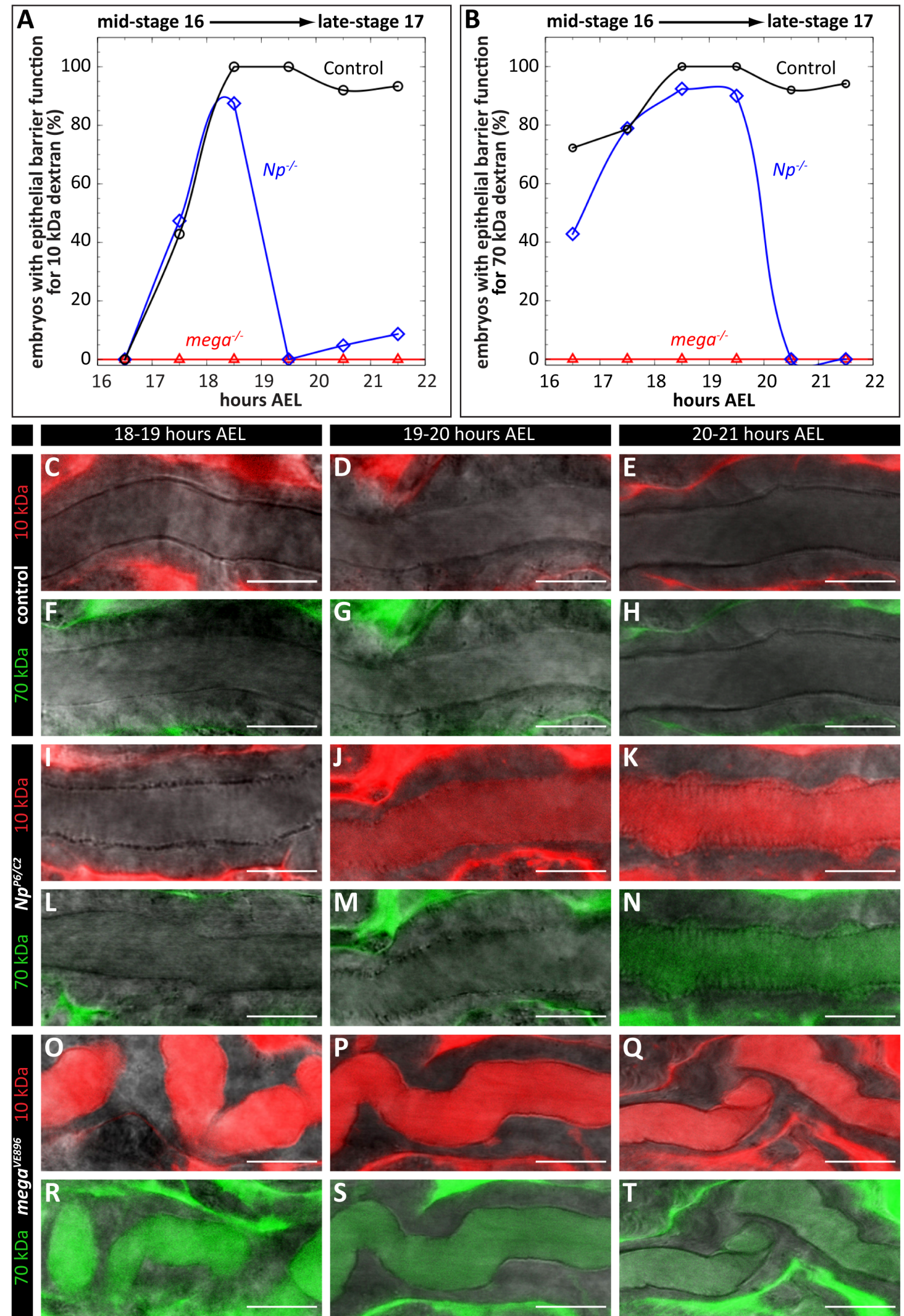

\section{Figure 12: Tracheal barrier function maintenance is dependent on Notopleural}

(A-B) Quantification of embryos with a tracheal barrier function for Texas Red-labeled 10 $\mathrm{kDa}$ dextran (A) and FITC-labeled $70 \mathrm{kDa}$ dextran (B) at different time points AEL. Control embryos (black graph; mega ${ }^{V E 896} /$ mega $^{+}$) establish a tracheal barrier function for both 10 
$\mathrm{kDa}$ and $70 \mathrm{kDa}$ dextran until 18.5 hours AEL. $N p^{P 6} / N p^{C 2}$ mutant embryos (blue graph) similarly establish a tracheal barrier function for both $10 \mathrm{kDa}$ and $70 \mathrm{kDa}$ dextran but do not maintain it until late-stage 17. No establishment of a tracheal barrier function is detectable in mega ${ }^{V E 896} / \mathrm{mega}^{\mathrm{VE} 896}$ mutant embryos (red graph). For each indicated stage: $\mathrm{n}=8$ for mega; $\mathrm{n}=15$ for $N p$ and control. (C-T) In vivo confocal LSM images of tracheal dorsal trunks of control (C-H), Np mutant (I-N) and mega mutant (O-T) embryos after injection of Texas Red-labeled $10 \mathrm{kDa}$ dextran (C-E, G-K, M-Q, S-T) and/or FITC-labeled $70 \mathrm{kDa}$ dextran (D-H, J-N, P-T) into the haemocoel at indicated hours AEL. Lack of tracheal barrier function is visible by presence of fluorophore-labeled dextran in the tracheal lumen (J, K, N, O-T). Scale bars correspond to $10 \mu \mathrm{m}$.

during different developmental stages (Fig 12). In control embryos, the tracheal barrier function is established during stage 16 at approximately 16-18 hours after egg laying (AEL; Fig 12A-B). Control embryos show a tracheal barrier function for $10 \mathrm{kDa}$ and $70 \mathrm{kDa}$ dextran at all tested developmental stages after 18 hours AEL (Fig 12A-H). Similar to control embryos, $N p$ mutant embryos establish a tracheal barrier function for $10 \mathrm{kDa}$ and $70 \mathrm{kDa}$ dextran at 16-18 hours AEL (Fig 12A-B). At 18-19 hours AEL, approximately $90 \%$ of $N p$ mutant embryos display a functional tracheal transepithelial barrier (Fig 12A-B, I, L). However, the barrier function is not maintained during subsequent embryogenesis. $N p$ mutant embryos lack tracheal barrier function for $10 \mathrm{kDa}$ dextran at 19-20 hours AEL (Fig 12A, J), and for $70 \mathrm{kDa}$ dextran at 20-21 hours AEL (Fig 12B, N). In contrast to control and $N p$ mutant embryos, mega mutant embryos did not establish a tracheal transepithelial barrier during embryogenesis. This was evident by diffusion of $10 \mathrm{kDa}$ and $70 \mathrm{kDa}$ dextran into the tracheal lumen at all tested developmental stages (Fig 12A-B, OT). These experiments indicate that the tracheal transepithelial barrier function of $N p$ mutant embryos is initially established, but it is not maintained during stage 17 of embryogenesis. This phenotype is distinct from mega mutant embryos that lack septate junction formation and establishment of a tracheal transepithelial barrier.

\subsubsection{Notopleural mediates tracheal apical extracellular matrix maturation}

The transient chitin cable that is present during developmental stages 15 and 16 in the tracheal lumen (Fig 9A-B) is degraded during late-stage 16 and early stage 17 (Fig 1E). At the same time, the mature tracheal aECM and cuticle respectively, 
forms on the apical surface of the tracheal cells (Fig 1D-E; Öztürk-Çolak et al. 2016b; Moussian et al. 2006a). The localization of $\mathrm{Np}$ in the tracheal intraluminal aECM in stages 16 and 17 and at the apical surface of tracheal cells in stage 17 (Fig 8E-F") suggests that it might play a role in tracheal aECM remodeling. To analyze the mature tracheal aECM of $N p$ mutant embryos, whole-mount stainings of chitin in mid-stage 17 embryos were performed.

In wild-type and $N p$ mutant embryos, degradation of the transient luminal chitin cable is completed in mid-stage 17 (Fig 13A-B), indicating that $\mathrm{Np}$ is not required for luminal chitin degradation. In this developmental stage, chitin is only present in the procuticle layer of the mature aECM that forms a distinct structure of taenidial folds in the tracheal system (Fig 1D-E; Öztürk-Çolak et al. 2016b). In wild-type embryos, these taenidial folds run perpendicular to the tube length in a regularly-spaced helical structure, visible as dots in a cross-sectional view (Fig 13A) and as parallel strands in a z-projection image of the trachea (Fig 13C). This taenidial folds structure is highly disrupted in $N p$ mutant embryos. The chitin strands are not arranged in parallel but intersect and merge frequently (Fig 13B, D). To quantify this phenotype, diameters of single chitin strands and the frequency of chitin strands along the longitudinal tube axis were measured in wildtype and $N p$ mutant dorsal trunks (Fig 13E). On average, the wild-type tracheal aECM contains 21 chitin strands per $10 \mu \mathrm{m}$ dorsal trunk length and the chitin strands have an average diameter of $0,32 \mu \mathrm{m}$ (Fig 13E). In contrast, $N p$ mutant dorsal trunks show an average of only 11 chitin strands per $10 \mu \mathrm{m}$ dorsal trunk length and highly increased diameters of the chitin strands (average: 0,49 $\mu \mathrm{m}$; Fig 13E). Furthermore, chitin strands show a non-uniform spacing in $N p$ mutant trachea. Signal-intensity profiles of chitin stainings along the longitudinal axis of dorsal trunks show a uniform frequency of intensity peaks (indicating taenidial folds) in wild-type embryos but lack a comprehensive pattern in $N p$ mutant embryos (Fig 13F).

While chitin in the procuticle layer is a major component of the mature tracheal aECM, the taenidial folds structure also comprises a layer of aECM proteins (epicuticle) and a hydrophobic layer of lipids (envelope; Moussian et al. 2006a). To visualize the overall structure of the tracheal aECM in $N p$ mutant embryos, an ultrastructural analysis by transmission electron microscopy was performed. The 

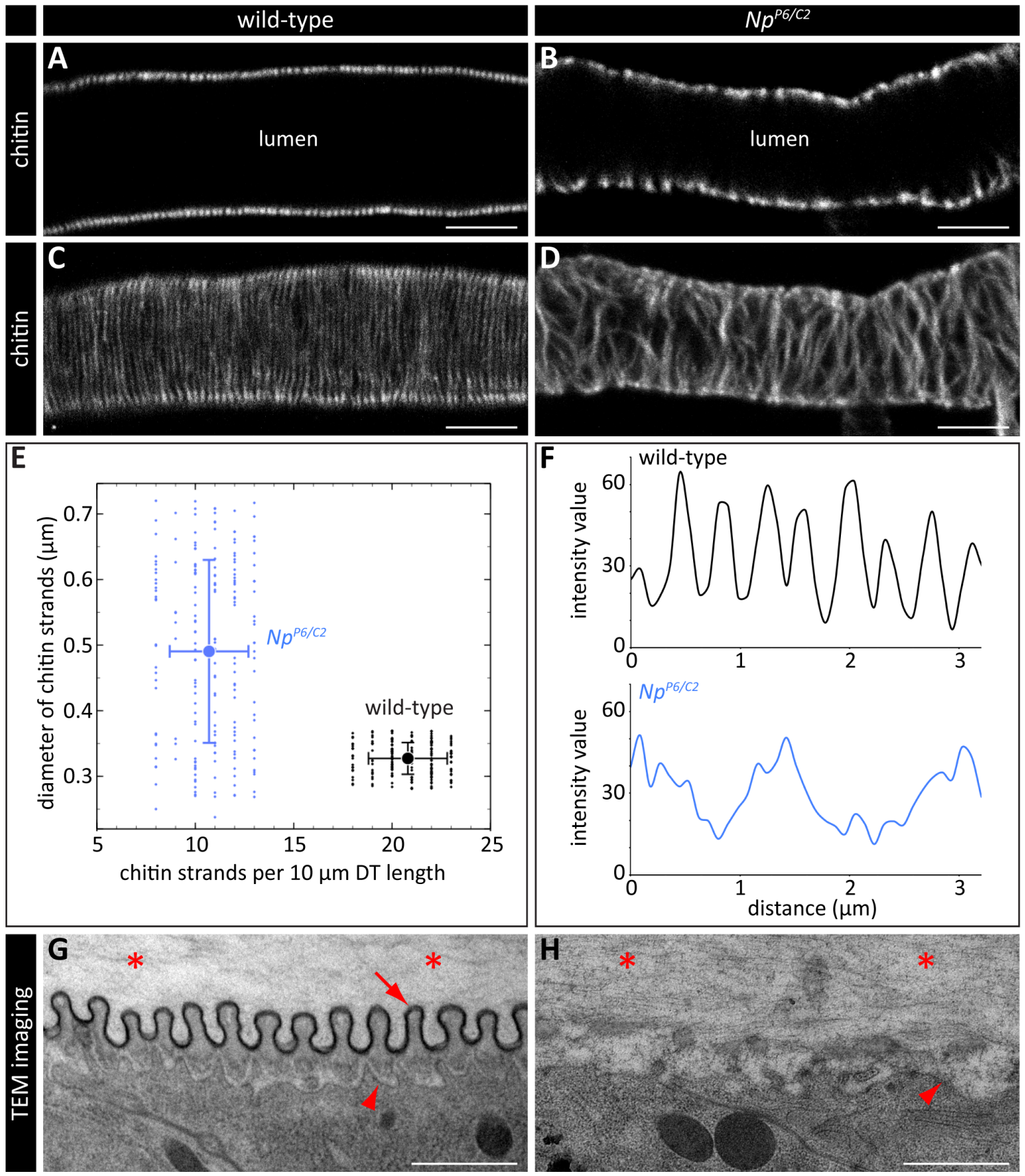

Figure 13: Notopleural is required for maturation of the tracheal apical extracellular matrix

(A-D) Confocal LSM single-plane images (A-B) and z-stack projections (C-D) of tracheal dorsal trunks of wild-type (A, C) and $N p^{P 6} / N p^{C 2}$ mutant (B, D) embryos stained with CBP (chitin). Scale bars correspond to $5 \mu \mathrm{m}$. (E) Quantification of diameter and frequency of stage 17 dorsal trunk chitin strands. Data represent measurements in $10 \mathrm{~Np} p^{P 6} / \mathrm{Np}^{C 2}$ mutant embryos and 10 wild-type embryos. For method details see chapter 4.3.11. (F) Representative intensity profiles of chitin stainings along the longitudinal axis of tracheal dorsal trunks of stage 17 wild-type and $N p$ mutant embryos. Peaks indicate chitin strands and taenidial folds, respectively. (G-H) Transmission electron microscopy (TEM) images of tracheal aECM in stage 17 wild-type $(\mathrm{G})$ and $N p^{P 6} / N p^{C 2}$ mutant $(\mathrm{H})$ embryos. The envelope (arrow) of the taenidial folds, apical plasma membrane (arrowheads) and tracheal lumen (asterisks) are clearly distinguishable in wild-type trachea (G). Np mutant embryos lack taenidial folds structure and show accumulation of electron dense material in the tracheal lumen (H). Scale bars correspond to $1 \mu \mathrm{m}$. 
tracheal aECM of mid-stage 17 wild-type embryos is characterized by evenly spaced taenidial folds (Fig 13G). The electron-dense envelope (arrow in Fig 13G), the subjacent procuticle layer, underlying apical plasma membrane (arrowhead in Fig 13G) and tracheal cells are visible and clearly distinguishable from the tracheal lumen (asterisks in Fig 13G). The thin epicuticle layer is not distinguishable from the larger procuticle layer in the taenidial folds, as reported in other studies (Moussian 2010; Moussian et al. 2006a). In contrast to wild-type taenidial folds, the tracheal mature aECM of $N p$ mutant embryos is highly unstructured and lacks taenidial folds organization (Fig 13H). Only rudimentary aECM material is visible on the extracellular side of the apical plasma membrane (arrowhead in Fig 13H) and no electron-dense envelope layer is detectable (Fig 13H).

These data indicate that $N p$ is essential for tracheal aECM maturation and taenidial folds morphogenesis, respectively. The $N p$ mutant phenotype suggests that $N p$ is required for stratified organization and taenidial folds patterning of the tracheal aECM, while degradation of the intraluminal chitin cable and chitin deposition in the mature tracheal aECM are not dependent on $N p$.

\subsubsection{Notopleural is essential for epidermal cuticle organization}

Besides the tracheal system, epithelia of the epidermis, hindgut and foregut are known to form a cuticle (Moussian et al. 2006a). The previous observations that $\mathrm{Np}$ is expressed in the embryonic epidermis (chapter 2.1.3) and $N p$ mutant embryos do not hatch as larvae (chapter 2.1.2) suggested that Np might also play a role in epidermal cuticle formation. The epidermal cuticle of late-stage $17 \mathrm{D}$. melanogaster embryos is highly similar to the tracheal cuticle. They share the same chitin-rich composition and stratified structure, but the epidermal cuticle has no taenidial folds pattern (Moussian et al. 2006a). Instead, the epidermal cuticle shows an overall smooth surface and segmentally repeated ventral belts of hair-like structures, the so-called denticle belts (Fernandes et al. 2010). To analyze the epidermal cuticle of $N p$ mutant embryos, chitin stainings and cuticle preparations of late-stage 17 embryos were performed (Nüsslein-Volhard et al. 1984).

The denticle belts of wild-type cuticles are visible as segmentally repeated areas of hair-like aECM protrusions on the ventral side (Fig 14A-A'). In contrast, $N p$ 

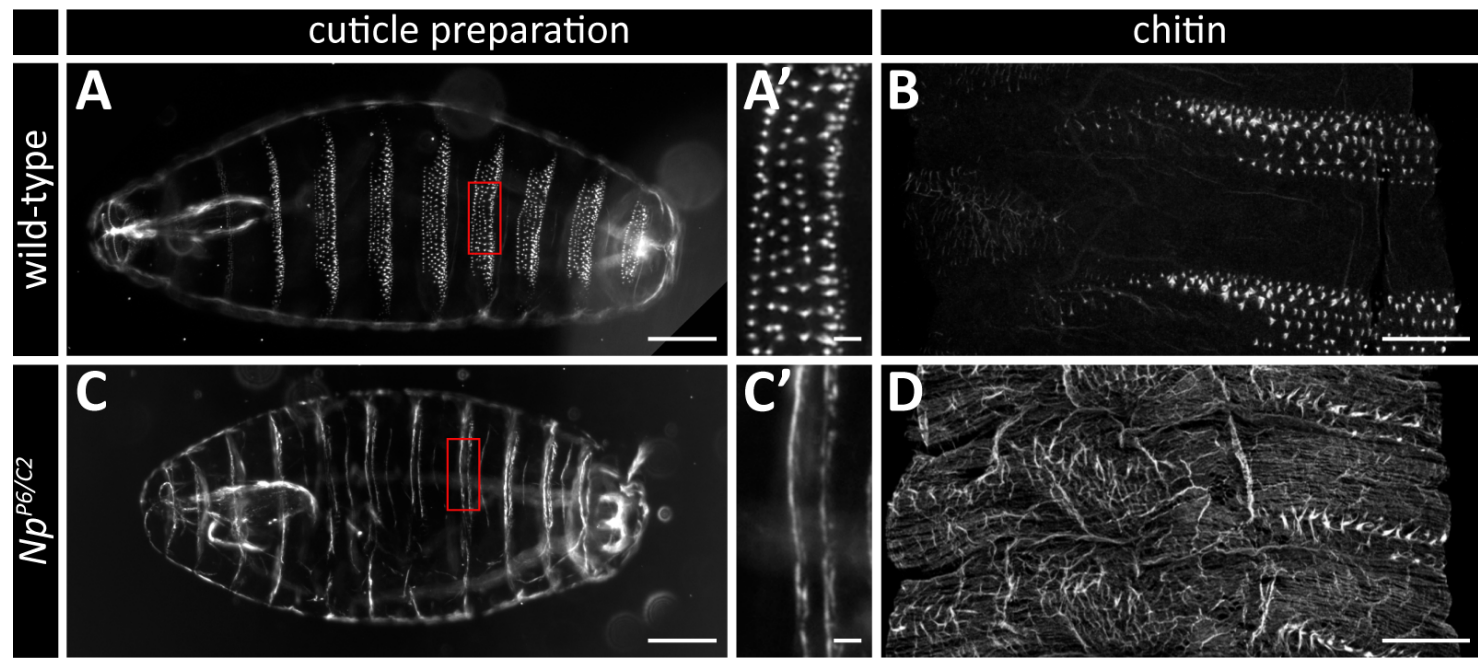

Figure 14: Notopleural is essential for epidermal cuticle organization

(A-A', C-C') Dark field light microscopic images of wild-type (A-A') and $N p^{P 6} / N p^{C 2}$ mutant (C-C') cuticle preparations imaged from ventral. Details of denticle belts ( $\left.\mathrm{A}^{\prime}, \mathrm{C}^{\prime}\right)$ correspond to the red frames in overview images $(\mathrm{A}, \mathrm{C})$ Denticle belts develop only rudimentary in $N p$ mutant embryos (C-C'). Scale bars correspond to $50 \mu \mathrm{m}(\mathrm{A}, \mathrm{C})$ and $5 \mu \mathrm{m}\left(\mathrm{A}^{\prime}, \mathrm{C}^{\prime}\right)$, respectively. (B, D) Confocal LSM images of abdominal segments 5-6 of stage 17 wild-type (B) and $N p^{P 6} / N^{C 2}$ mutant (D) embryos stained with FITC-labeled CBP (chitin). Images show a lateral view. Ventral is to the right and posterior to the top. In wild-type embryos (B), chitin is enriched in the denticles, while $N p$ mutant embryos (D) show aberrant chitin depositions throughout the epidermal cuticle. Scale bars correspond to $30 \mu \mathrm{m}$.

mutant cuticles display deformed ridges on their ventral side that presumably correspond to rudimentary denticle belts (Fig 14C-C'). While these ventral structures are also segmentally repeated (Fig 14C), they rarely contain structures that resemble the shape of wild-type denticles (Fig 14C'). In addition to rudimentary denticle belt formation, chitin deposition in the epidermal cuticle is aberrant in $N p$ mutant embryos. Wild-type embryos show an enrichment of chitin only in the ventral denticles (Fig 14B). In contrast, the cuticle of $N p$ mutant embryos shows abnormal chitin depositions throughout its entire surface (Fig 14D). Thus, epidermal cuticle organization and denticle belt formation are dependent on $N p$. In addition to the role of $N p$ in tracheal aECM organization, this suggests a general regulatory role of $\mathrm{Np}$ in $D$. melanogaster embryonic aECM maturation. 


\subsubsection{Intraluminal degradation of the zona pellucida domain protein Dumpy depends on Notopleural}

In comparison to wild-type trachea, electron micrographs of $N p$ mutant trachea show notably increased amounts of electron-dense material in the tracheal lumen (Fig 13G-H). This might indicate a failure in clearance of intraluminal aECM components. Since the chitin cable is properly removed from the tracheal lumen in $N p$ mutant embryos (Fig 13B), the remaining material is presumably proteinaceous. A well-established component of the tracheal aECM is the ZPD protein Dpy (Dong and Hayashi 2015). Dpy is a constituent of the transient luminal aECM cable and also integrated into the mature tracheal aECM. It is one of the largest known proteins (2,5 MDa) and presumably polymerizes via its ZPD in a homo- and heterophilic manner into elastic fibrils (Dong et al. 2014 ; Jovine et al. 2002; Wilkin et al. 2000). To analyze Dpy localization in the tracheal system, a dpy::YFP transgene was used that generates expression of a Dpy::YFP fusion protein from the endogenous locus (Lye et al. 2014).

Dpy::YFP localizes to the tracheal intraluminal aECM cable in stage 16 embryos (Fig 15A-A"). Enrichment of Dpy::YFP at the core (arrowhead in Fig 15A") and at the peripheral region (arrow in Fig 15A") of the chitin cable are distinguishable. In early-stage 17, the intraluminal aECM cable contracts and the mature tracheal aECM starts to form (Fig 15B-B"). In this stage, chitin is present in both, the intraluminal aECM cable and the developing taenidial folds (Fig 15B'), while Dpy::YFP is only present in the intraluminal aECM cable (Fig 15B"). Degradation of tracheal intraluminal Dpy::YFP and chitin is completed in dpy::YFP embryos at mid-stage 17, visible by absence of Dpy::YFP and chitin in the intraluminal space (Fig 15C-C"). Notably, Dpy::YFP also localizes to the mature tracheal aECM in this stage (Fig 15C, C"). Stage $16 \mathrm{~Np}$ mutant embryos show a wild-type-like incorporation of Dpy::YFP into the tracheal intraluminal chitin cable (Fig 15D-D"), displaying Dpy::YFP enrichment at the core (arrowhead in Fig 15D") and peripheral region (arrow in Fig 15D") of the aECM cable. Similar to wild-type embryos, formation of the mature tracheal aECM is visible in early-stage $17 \mathrm{~Np}$ mutant embryos (Fig 15E-E'). However, no contraction of the intraluminal aECM cable is detectable. While intraluminal chitin is present at lower levels than in stage $16 \mathrm{~Np}$ mutant embryos, which indicates chitin degradation, the chitin cable is spread across the entire transverse luminal axis (Fig 15E-E'). Furthermore, 

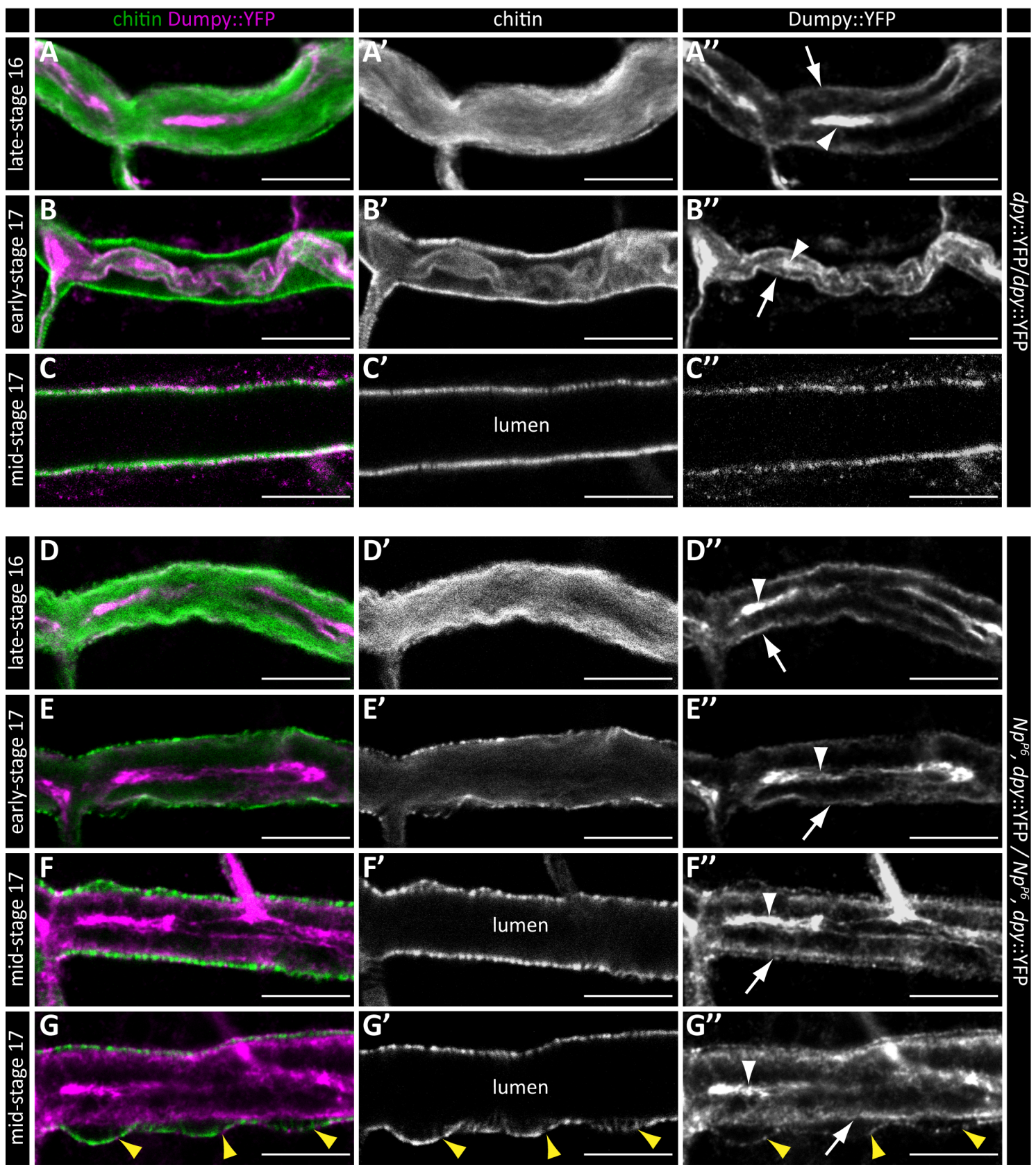

Figure 15: Notopleural is required for tracheal intraluminal Dumpy degradation.

Confocal LSM images of dorsal trunks of $d p y:$ YFP/dpy::YFP (A-C") and $N p^{P 6}, d p y:$ YFP/ $N p^{P 6}, d p y:$ :YFP mutant (D-G”) embryos at late-stage 16 (A-A”, D-D”), early-stage 17 (B-B", E-E") and mid-stage 17 (C-C", F-G") stained with CBP (chitin; green) and anti-GFP antibodies (magenta). Intraluminal Dpy::YFP forms a central core (white arrowheads) and a peripheral "shell" layer (arrows) in the chitin cable of late-stage 16 wild-type-like trachea (A-A") and $N p$ mutant trachea (D-D"). In $d p y:$ YFP embryos, luminal chitin and Dpy::YFP contract at early-stage 17 (B-B"), and chitin and Dpy::YFP are removed from the lumen until mid-stage 17 (C-C"). $N p$ mutant embryos show no contraction of the luminal chitin and Dpy::YFP cable during early-stage 17 (E-E") and no luminal clearance of Dpy::YFP during mid-stage 17 (F-G"). Chitin is cleared normally from the lumen of $N p$ mutant trachea in mid-stage 17 (F', G'). While the intraluminal Dpy::YFP cable remains attached to the chitin of the mature aECM in most areas of $N p$ mutant trachea, contact is lost in randomly distributed bulges (yellow arrowheads in G-G"). Scale bars correspond to $10 \mu \mathrm{m}$. 
peripheral Dpy::YFP of the intraluminal cable (arrow in Fig15E") co-localizes with the forming mature aECM and does not contract (Fig 15E, E"). In mid-stage $17 N p$ mutant embryos, clearance of the intraluminal chitin cable is completed (Fig 15F$\mathrm{F}^{\prime}$ ), while the intraluminal Dpy::YFP cable is still present, showing no signs of degradation or contraction (Fig 15F, F"). The central Dpy::YFP core (arrowhead in Fig 15F") is clearly distinguishable from peripheral Dpy::YFP (arrow in Fig15F") that remains associated to the misorganized chitin of the mature aECM in most areas of the mid-stage $17 \mathrm{~Np}$ mutant tracheal system (Fig 15F-F"). However, contact between peripheral Dpy::YFP and the chitin strands is lost in randomly distributed areas (Fig 15G-G"). These areas are visible as bulges of the mature tracheal aECM (yellow arrowheads in Fig 15G-G") in which peripheral Dpy::YFP of the intraluminal aECM cable (arrow in Fig 15G") is clearly separated from the misorganized chitin strands (Fig 15G-G"). Notably, Dpy::YFP is also integrated into the misorganized $N p$ mutant mature tracheal aECM (Fig 15E-G"), which is prominently visible in tracheal bulges (yellow arrowheads in Fig 15G").

These results indicate, that degradation of intraluminal Dpy and separation of the intraluminal aECM cable from the forming mature aECM depend on Np. This also supports a role of $\mathrm{Np}$ in regulation of cell - matrix interactions at the apical side of tracheal cells, as seen in earlier developmental stages (Fig 10E-H").

\subsubsection{Notopleural regulates attachment of apical extracellular matrix to the apical plasma membranes in the tracheal system}

To analyze the interactions between tracheal aECM and apical cellular membranes during aECM maturation, late-stage 16 to mid-stage 17 embryos were stained for Uif (apical membrane marker) and chitin. In wild-type embryos, chitin of the forming mature tracheal aECM is in direct contact with the apical plasma membrane from late-stage 16 to mid-stage 17 (Fig 16A-C"). As shown before, the intraluminal chitin cable is separated from the apical cellular surface by a small gap in most areas of late-stage 16 tracheal dorsal trunks (Fig 16A-A"). The cable then contracts during early-stage 17 (Fig 16B-B") and is completely removed from the tracheal lumen in mid-stage 17 (Fig 16C-C"). In $N p$ mutant embryos, the intraluminal chitin cable is in abnormally close contact to the tracheal apical cell membranes in most areas during stage 16 (Fig 16D-D”; see also Fig 10E-H”). 

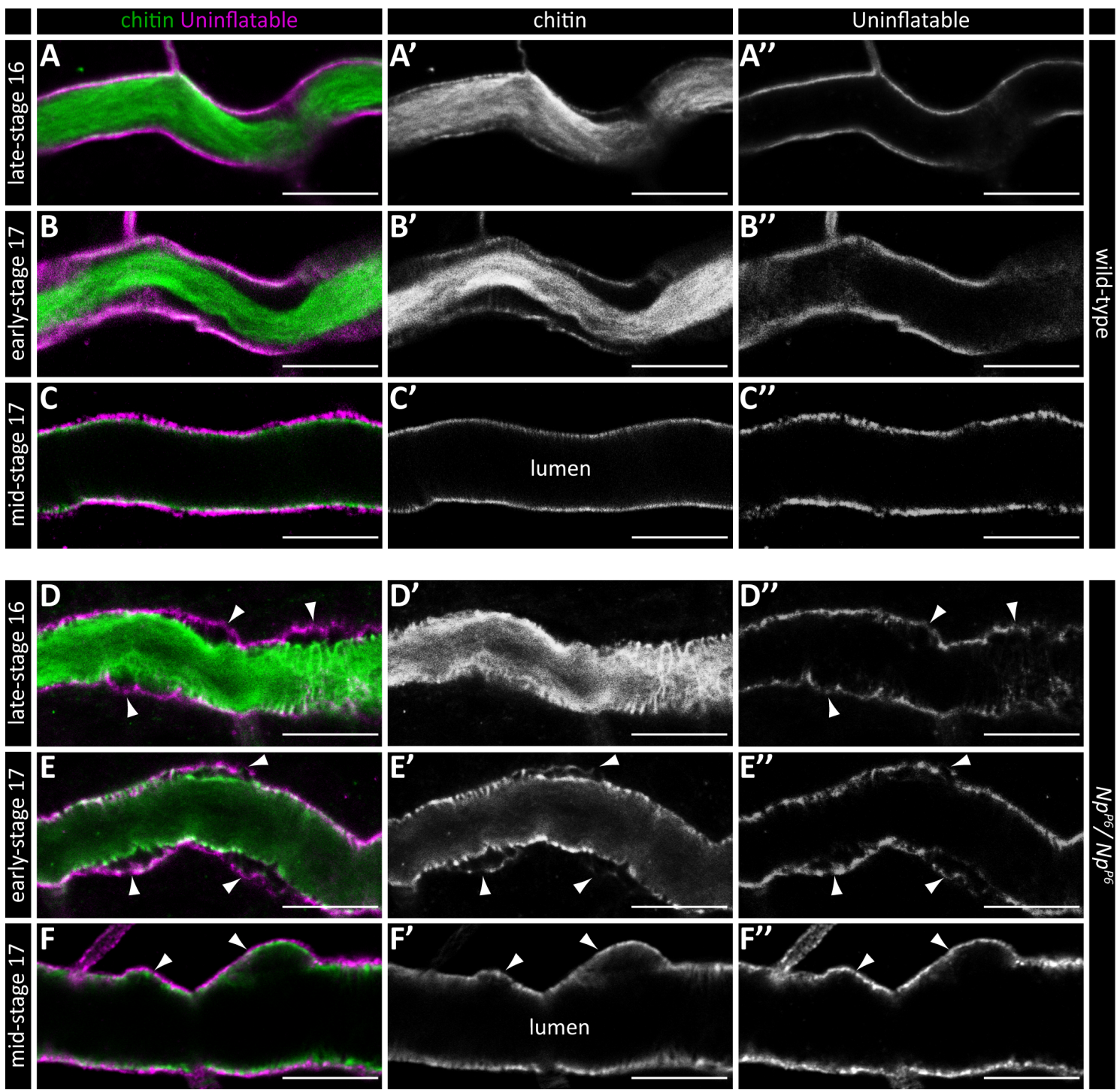

Figure 16: Notopleural regulates apical extracellular matrix - cell interactions in the tracheal system

Confocal LSM images of dorsal trunks of wild-type (A-C") and $N p^{P 6} / N p^{C 2}$ mutant (D-F") embryos stained with CBP (chitin; green) and anti-Uif antibody (magenta). In wild-type embryos, the tracheal apical cell membranes (Uif) are in contact with the forming mature aECM at all shown stages (A-C"). In $N p$ mutant embryos, the apical cell membranes are detached from the forming mature aECM in some areas during late-stage 16 (arrowheads in D, D"). A second layer of misorganized aECM forms in these bulges during early-stage 17 (arrowheads in E-E") and the bulges are still visible in mid-stage $17 \mathrm{~Np}$ mutant embryos (arrowheads in F-F"'). Scale bars correspond to $10 \mu \mathrm{m}$.

However, the apical plasma membranes form bulges in which they are separated from the intraluminal chitin cable and the forming mature aECM (arrowheads in Fig 16D, D"). These bulges are also present during chitin cable degradation in early-stage $17 \mathrm{~Np}$ mutant embryos (arrowheads in Fig 16E-E"). Here, chitin of the 
mature aECM accumulates on the apical plasma membranes in separation from the chitin cable and another layer of chitin accumulation is present at the margin of the intraluminal chitin cable. While separation of mature aECM from the contracted intraluminal chitin cable is observable in the tracheal system of earlystage 17 wild-type embryos (Fig 16B-B"), enrichment of chitin at the aECM cable margin was never observed. In mid-stage $17 \mathrm{~Np}$ mutant embryos, degradation of the intraluminal chitin cable is completed and chitin localizes to the mis-organized mature aECM on the apical cellular surface (Fig 16F-F”). Bulges are still present at this stage (arrowheads; Fig 16F-F").

While Figure 16D-F" shows selected $N p$ mutant tracheal dorsal trunks with bulges, the frequency, size and distribution of bulges followed no comprehensive pattern. Furthermore, antibody stainings in fixed embryos only show a snapshot of tracheal development, with no information regarding the dynamics of bulge formation. To observe $N p$ mutant tracheal cells during aECM maturation in vivo, $N p$ mutant embryos with pan-tracheal expression of UAS- $\alpha$-tubulin::GFP were used. Tracheal dorsal trunks were imaged in time intervals of $10 \mathrm{~min}$ for more than two hours, starting at 20 hours AEL (early-stage 17). In the shown dorsal trunk section of an $N p$ mutant embryo, two bulges formed during this time period (Fig 17). At the beginning of the time series, the forming mature aECM (dashed line) is in direct contact with the a-tubulin::GFP expressing tracheal cells (green; Fig 17B-C). The bulges then grow steadily during the following two hours, visible by separation of the tracheal cells from the forming misorganized mature aECM (Fig 17B-C). Therefore, bulges in $N p$ mutant tracheal dorsal trunks are rigid structures that form irreversibly during stage 17 . The observations furthermore suggest that proper attachment of chitin depositions of the mature aECM to apical tracheal membranes depends on $\mathrm{Np}$. 
A

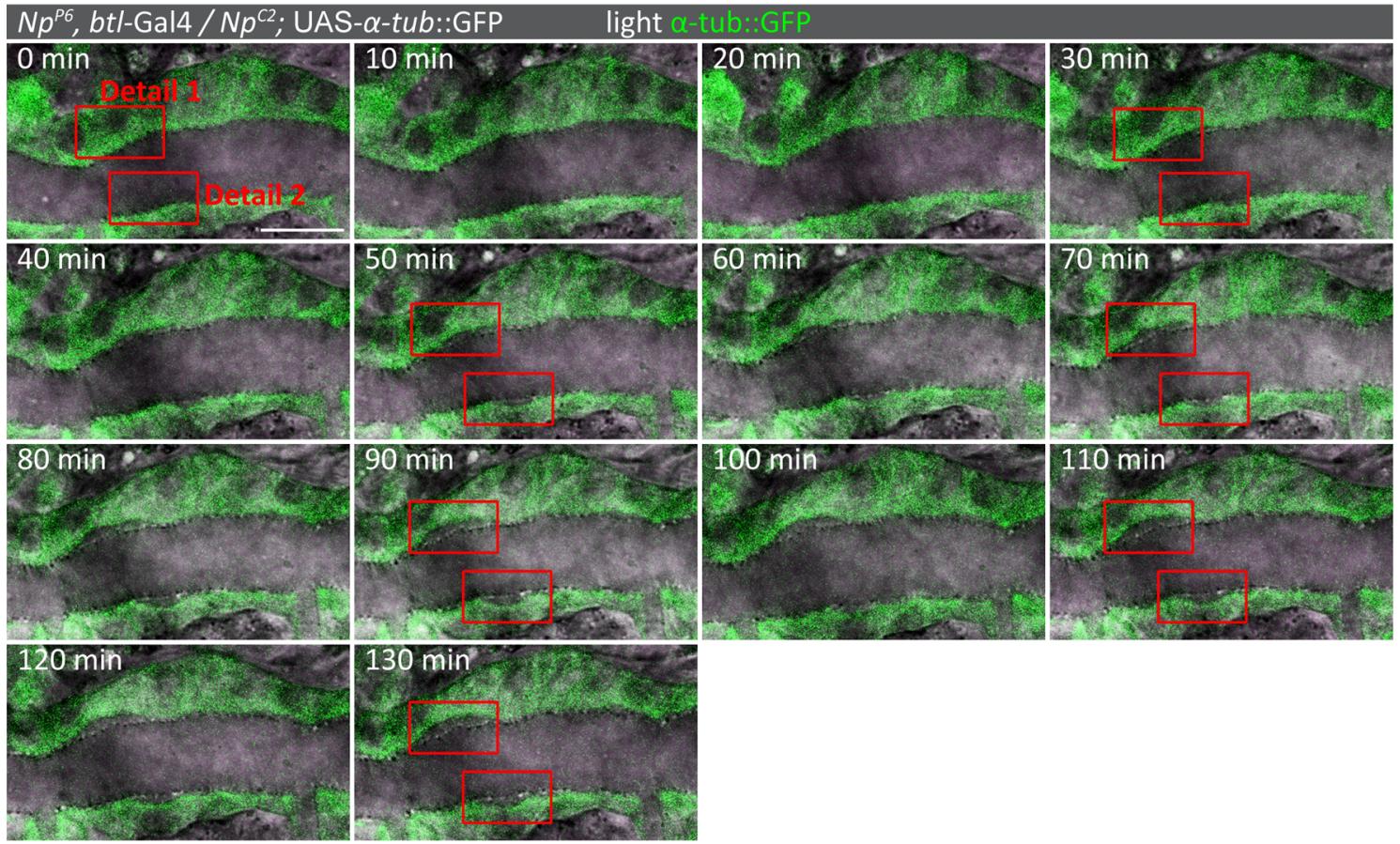

B

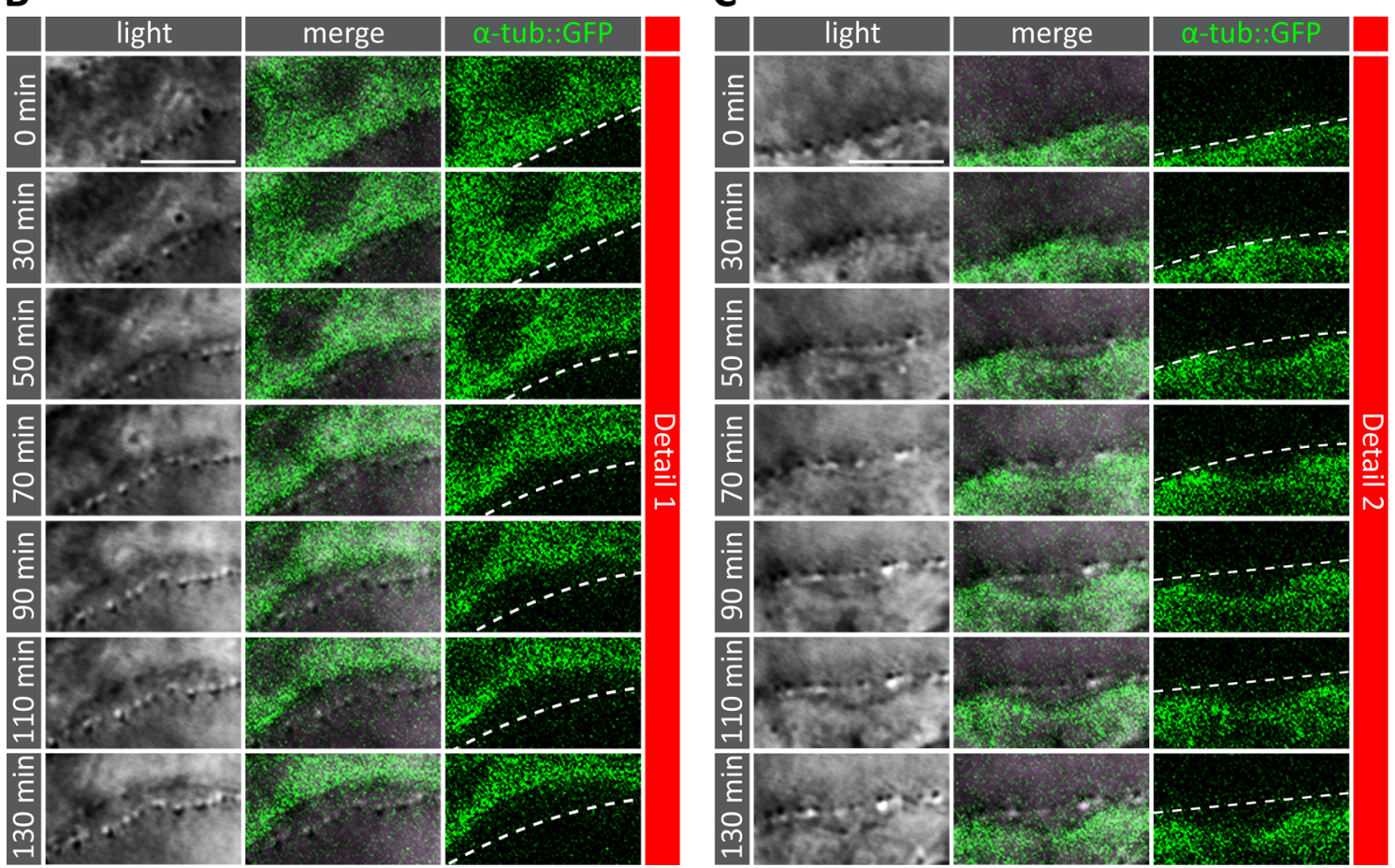

Figure 17: Bulges in Notopleural mutant trachea are rigid structures

In vivo confocal LSM time-series images of a tracheal dorsal trunk of a $\mathrm{Np}^{P 6}$, btlGal4/N $p^{C 2}$;UAS- $\alpha$-tubulin::GFP embryo showing $\alpha$-tub::GFP (green) and transmitted light channels. Detail 1 images (B) and detail 2 images (C) correspond to the red frames in overview images (A). Two bulges (B-C) form in the observed dorsal trunk segment (A) during 130 min of observation, visible by separation of the tracheal cells (green) from the forming mature aECM (dashed line in B-C). Scale bars correspond to $10 \mu \mathrm{m}$ (A) and 3,5 $\mu \mathrm{m}$ (B-C), respectively. 


\subsubsection{Release of the zona pellucida domain protein Piopio from the tracheal apical cell surface is mediated by Notopleural}

Besides Dpy, the ZPD protein Pio is a known component of the transient intraluminal tracheal aECM cable. Interestingly, Pio has been proposed to play a role in aECM attachment to the apical plasma membranes in the tracheal system due to its transmembrane domain and extracellular ZPD (Öztürk-Çolak et al. 2016b). Furthermore, Pio is essential for cell intercalations in tracheal branches that develop from multicellular tubes to unicellular tubes with autocellular junctions (Jazwinska et al. 2003). Dpy has a similar function during tracheal morphogenesis and both, Dpy and Pio mediate attachment of apodemes to the cuticle (Bökel et al. 2005; Wilkin et al. 2000). Therefore, it was proposed that aberrant aECM - cell attachments in $N p$ mutant embryos may be caused by misregulation of proteolytic processing or localization of Pio.

To visualize Pio localization in the developing embryo, flies that endogenously express a mCherry::Pio fusion protein were generated using CRISPR/Cas9 mediated homology-directed repair (Fig 18). The mCherry encoding sequence and a 3xP3-DsRed marker gene were inserted into the third protein-coding exon of pio (Fig 18A-B). The 3xP3-DsRed marker gene was then removed by PiggyBac transposase-mediated excision, generating the mCherry::pio allele that lacks the marker gene and the PiggyBac transposon ends (Fig 18C). In the encoded mCherry::Pio fusion protein, mCherry is inserted carboxy-terminal of the signal peptide in the extracellular part of Pio (Fig 18D).

To visualize mCherry::Pio expression during embryogenesis, whole-mount antibody stainings of mCherry::pio embryos with anti-mCherry antibodies were performed. Similar to Np (Fig 8), mCherry::Pio is expressed in ectodermally derived tissues during embryonic development (Fig 19). It is detectable in the tracheal system, salivary glands, pharynx, hindgut, foregut, posterior spiracles and epidermis (Fig 19). In the tracheal system, mCherry::Pio is present during early tracheal development in stage 11 embryos (Fig 19A) and also detectable in stages 13 to 16 (Fig 19B-D). However, mCherry::Pio is not uniformly expressed in all tracheal branches and shows a dynamic developmental stage specific spatial distribution in the tracheal system. mCherry::Pio exclusively localizes to dorsal and ventral primary branches in stage 11 tracheal metameres (Fig 19A). Furthermore, in 


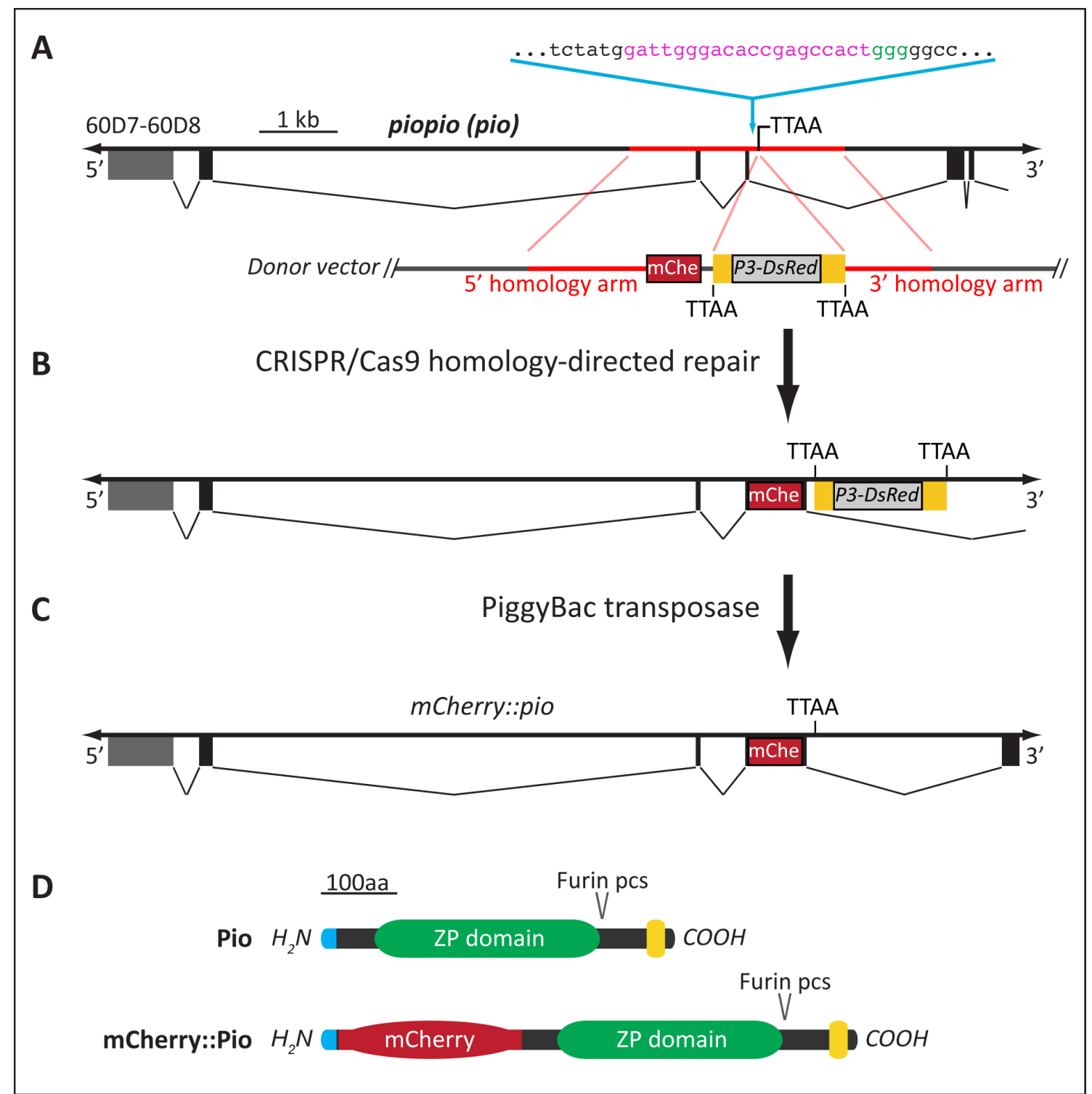

Figure 18: Generation of flies with endogenous expression of mCherry::Piopio using CRISPR/Cas9 technology.

(A) Schema of the 5' region of the pio genomic locus together with a donor vector containing two homology arms (red) and a mCherry encoding sequence (mChe) followed by a $3 x P 3$ DsRed marker gene flanked by PiggyBac transposon ends (yellow). The sgRNA recognition site (magenta letters), PAM (green letters), translated DNA (black boxes) and UTRs (grey boxes) are indicated. (B) pio locus after CRISPR/Cas9-mediated homology-directed repair. (C) mCherry::pio allele after PiggyBac transposase-mediated P3-DsRed marker gene excision. (D) Schemata of wild-type Pio and mCherry::Pio proteins. Signal peptides (blue), mCherry, ZP domains, Furin protease cleavage sites (pcs) and transmembrane domains (yellow) are indicated. 
dorsal branches of stage 13 and 14 embryos, mCherry::Pio is only present in the most dorsal cells (arrowheads in Fig 19B-C) and in the tracheal system of stage 16 embryos, mCherry::Pio predominately localizes to the dorsal trunk (Fig 19D).
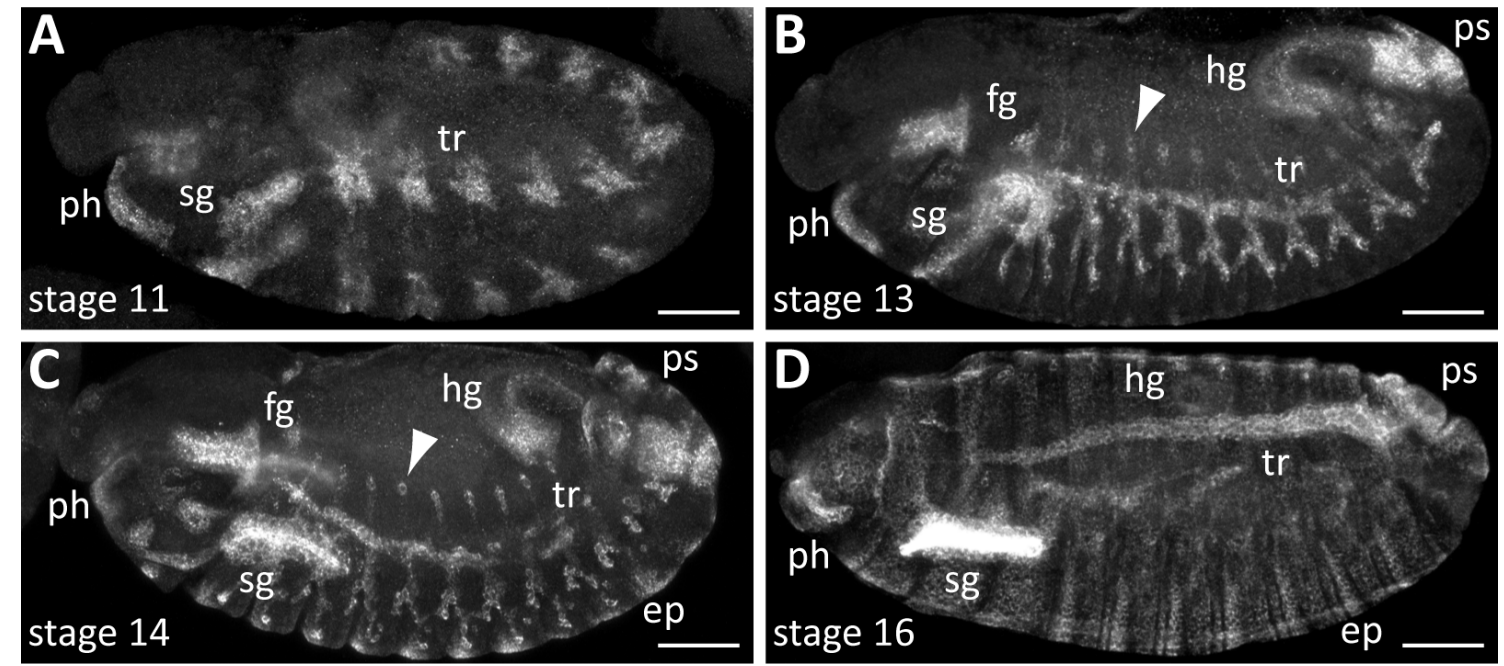

Figure 19: Expression pattern of mCherry::Piopio during embryogenesis

Confocal LSM z-stack projection images of whole-mount stainings of stage 11 (A), stage 13 (B), stage 14 (C) and stage 16 (D) mCherry::pio embryos with anti-mCherry antibodies. mCherry::Pio is detectable in the tracheal system (tr), the pharynx (ph) and the salivary glands (sg) in all shown stages. Expression is also detectable in the hindgut (hg), foregut (fg) and posterior spiracles (ps) from stage 13 onwards (B-D) and in the epidermis (ep) from stage 14 onwards (C-D). Localization of mCherry::Pio to dorsal branch tip cells is indicated by arrowheads (B-C). Scale bars correspond to $50 \mu \mathrm{m}$.

To analyze subcellular mCherry::Pio localization in the embryonic tracheal system, whole-mount stainings of mCherry::pio embryos with anti-mCherry antibodies and CBP were performed. In early stage 16, mCherry::Pio is present in tracheal cells, the intraluminal aECM cable and at the apical surface of tracheal cells (Fig 20AA"). Only low levels of mCherry::Pio are detectable in the intraluminal aECM cable in this stage (arrow in Fig 20A"). In late-stage 16, the localization pattern changes and mCherry::Pio is present in the intraluminal space and at the apical surface of tracheal cells in similar levels (Fig 20B-B"). This localization pattern continues during intraluminal aECM cable degradation in early-stage 17 (Fig 20C-C"). Together with intraluminal chitin, mCherry::Pio is then removed from the tracheal lumen until mid-stage 17 (Fig 20D-D"), similar to the observed removal of Dpy from the tracheal lumen (Fig 15B-C). In the tracheal system of early-stage $16 \mathrm{~Np}$ mutant embryos, mCherry::Pio localizes to the cytoplasm and apical surface of tracheal cells and in low levels to the tracheal lumen (Fig 20E-E"). This localization 

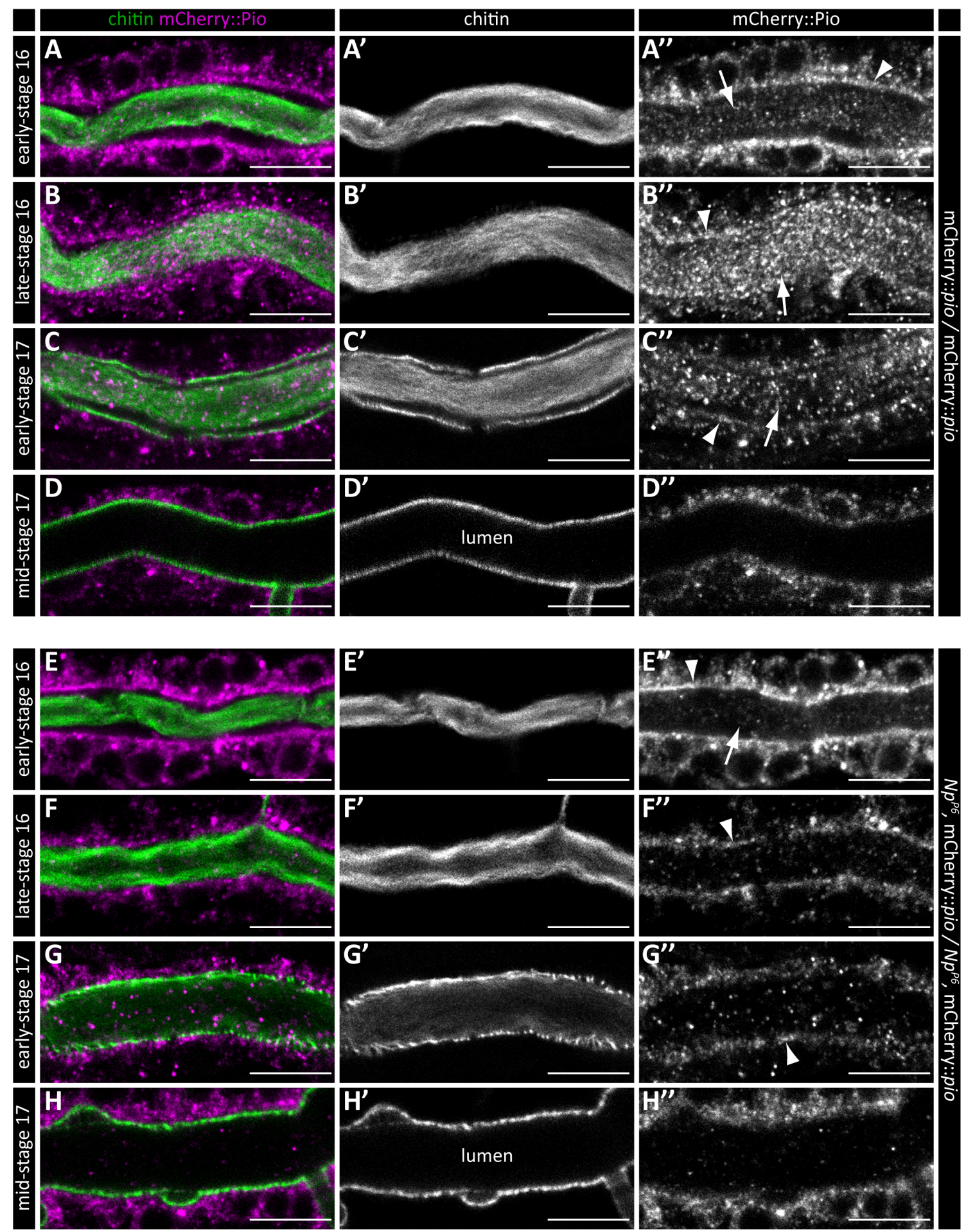

Figure 20: Release of Piopio from tracheal apical cell membranes is regulated by Notopleural

Confocal LSM images of tracheal dorsal trunks of mCherry::pio / mCherry::pio (A-D") and $N p^{P 6}$, mCherry::pio / $N p^{P 6}$, mCherry::pio mutant (E-H") embryos at early-stage 16 (A-A", EE"), late-stage 16 (B-B", F-F"), early-stage 17 (C-C", G-G") and mid-stage 17 (D-D", H-H") stained with CBP (chitin) and anti-mCherry antibodies (magenta). In wild-type-like embryos, mCherry::Pio is released into the lumen during late-stage 16 (B-B"). In $N p$ mutant embryos, mCherry::Pio is not released into the lumen in late-stage 16 (F-F") and remains associated to the apical membranes (G-G"). Arrows mark intraluminal localization and arrowheads apical membrane-associated localization. Scale bars correspond to $10 \mu \mathrm{m}$. 
pattern is similar to embryos with wild-type genetic background (Fig 20A-A"). However, in $N p$ mutant embryos, mCherry::Pio is not released into the tracheal lumen in late-stage 16 (Fig 20F-F"). Instead, it predominately localizes at the apical surface of tracheal cells (arrowhead in Fig 20F"). This apical membraneassociated localization of mCherry::Pio continues during chitin cable degradation in early-stage 17 (Fig 20G-G"). In mid-stage $17 \mathrm{~Np}$ mutant embryos, mCherry::Pio is only detectable in tracheal cells (Fig $20 \mathrm{H}-\mathrm{H}^{\prime \prime}$ ), similar to what has been observed in mCherry::pio embryos (Fig 20D-D").

These observations suggest that shedding of Pio from the tracheal apical plasma membranes during aECM maturation is regulated by Np. Since Pio might link the tracheal cells to the aECM, this would be consistent with the previously identified role of $\mathrm{Np}$ in regulation of cell - aECM attachment (chapter 2.1.9).

\subsubsection{Localization patterns of Dumpy and Piopio in the epidermis depend on Notopleural}

As previously shown, $\mathrm{Np}$ is essential for cuticle formation in the tracheal system (chapter 2.1.6) and epidermis (chapter 2.1.7). It was also shown that $\mathrm{Np}$ is required for degradation and normal localization of the aECM ZPD proteins Dpy and Pio during cuticle formation in the tracheal system (chapters 2.1.8 and 2.1.10). Besides their role in the tracheal system, Dpy and Pio are both implicated in attachment of the epidermal apodemes (muscle attachment sites) to the cuticle (Bökel et al. 2005; Wilkin et al. 2000). Since $\mathrm{Np}$ is also expressed in the epidermis (chapter 2.1.3), degradation and/or localization of Dpy and Pio in the epidermis may also be regulated by $\mathrm{Np}$. To analyze whether $\mathrm{Np}$ affects Dpy and Pio localization in the embryonic epidermis, whole-mount antibody stainings for Shortstop (Shot) and Dpy::YFP and mCherry::Pio, respectively, were performed in embryos with wildtype, and $N p$ mutant genetic background. Shot is a marker for the epidermal apodemes (Subramanian et al. 2003).

In mid-stage 17 wild-type-like control embryos, Dpy::YFP localizes to the epidermal

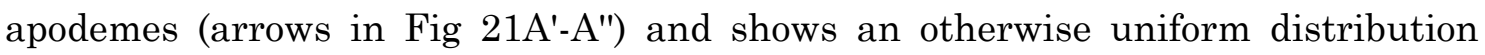
across the embryonic epidermis (Fig 21A-A"). In contrast, Dpy::YFP almost exclusively localizes to the apodemes in the epidermis of mid-stage $17 \mathrm{~Np}$ mutant 

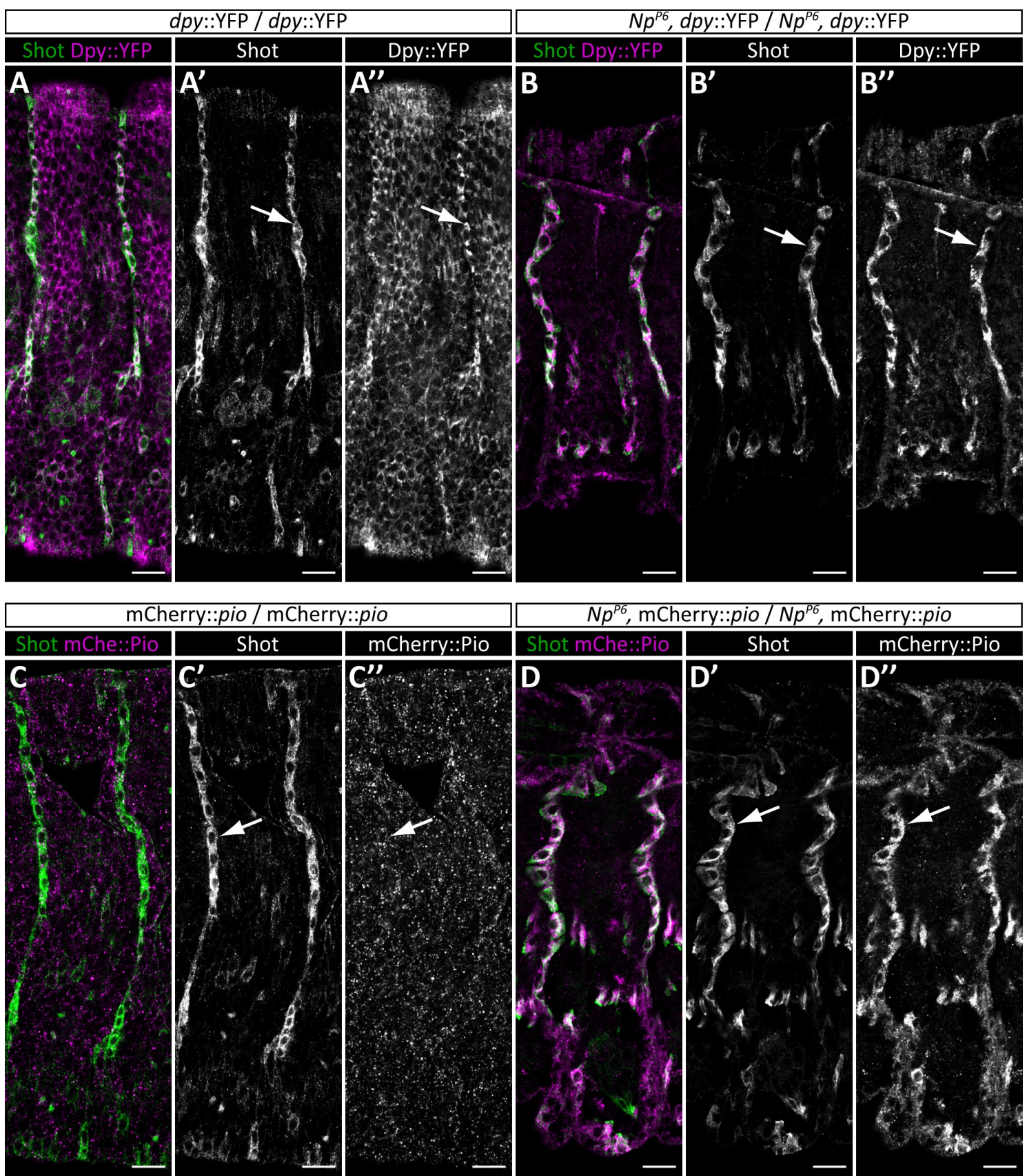

Figure 21: Epidermal localization patterns of Dumpy and Piopio are dependent on Notopleural

Confocal LSM images of the epidermis of abdominal segments 5-6 of stage $17 d p y:$ YFP / $d p y:$ YFP (A-A“), $N p^{P 6}$, dpy::YFP / $N p^{P 6}$, $d p y:$ YFP (B-B“), mCherry::pio / mCherry::pio (C$\mathrm{C}^{\prime \prime}$ ) and $N p^{P 6}$, mCherry::pio / $N p^{P 6}$, mCherry::pio (D-D“) embryos stained with anti-shortstop (shot; green) and anti-GFP (Dpy::YFP; magenta) antibodies (A-B“) or anti-shot (green) and anti-mCherry (mCherry::Pio; magenta) antibodies (C-D“). In embryos with wild-type genetic background, Dpy::YFP and mCherry::Pio show a uniform distribution in the epidermis and are also present at the apodemes (arrows, marked by shot; A-A“, C-C"). In contrast, Dpy::YFP and mCherry::Pio predominately localize to the epidermal apodemes in $N p$ mutant embryos and display no uniform distribution in the epidermis, respectively (BB“, D-D“). Scale bars correspond to $10 \mu \mathrm{m}$. 
embryos (arrows in Fig 21B-B"). Only very low levels of Dpy::YFP are detectable in other parts of the epidermis and do not co-localize with Shot, respectively (Fig 21B$\mathrm{B}^{\prime \prime)}$. Np similarly restricts localization of mCherry::Pio to the epidermal apodemes (Fig 21C-D"). In mid-stage 17 wild-type-like mCherry::pio embryos, a uniform epidermal distribution of mCherry::Pio is observable (Fig 21C-C"), showing no enrichments at epidermal apodemes (arrows in Fig $\left.21 \mathrm{C}^{\prime}-\mathrm{C}^{\prime \prime}\right)$. In contrast, mCherry::Pio shows a defined localization to the apodemes and co-localization with Shot in the epidermis of mid-stage $17 \mathrm{~Np}$ mutant embryos (arrows in Fig 21D-D"). Only very low levels of mCherry::Pio do not localize to the apodemes in the epidermis of these embryos (Fig 21D-D"). These findings indicate that Np mediates uniform distribution of Dpy and Pio in the embryonic epidermis during stage 17 cuticle maturation. Thus, regulation of cuticle maturation by $\mathrm{Np}$ in the tracheal system (chapter 2.1.6) and epidermis (chapter 2.1.7) is accompanied by $\mathrm{Np}$ mediated regulation of degradation and/or localization of Dpy and Pio in both tissues (chapters 2.1.8, 2.1.10 and this chapter).

\subsubsection{Proteolytic processing of Piopio is mediated by Notopleural}

While Np regulates localization patterns of Dpy and Pio in multiple tissues during embryogenesis (chapters 2.1.8, 2.1.10 and 2.1.11), it remained unclear whether this was due to proteolytic processing of Dpy and Pio by Np. Unfortunately, analysis of proteolytic processing of Dpy did not seem feasible since it is a 2,5 MDa protein with many post-translational modifications (Sakaidani et al. 2011; Wilkin et al. 2000). Therefore, analysis of proteolytic processing was done for Pio. To test the effect of $\mathrm{Np}$ on proteolytic processing of Pio, lysates of mCherry::pio embryos and $N p$ mutant embryos carrying the mCherry::piotransgene were prepared and analyzed by SDS-PAGE followed by western blotting with anti-mCherry (Fig 22A) and anti-B-tubulin (Fig 22B) antibodies. Embryos were collected at mid-stage 16 (before aECM maturation) and mid-stage 17 (during aECM maturation) and lysates were prepared under denaturing and reducing conditions. While lysates of wild-type embryos (negative control) show no immuno-reactive proteins in stainings with anti-mCherry antibodies (Fig 22A; lanes 1-2), three mCherry::Pio fragments are detectable in lysates of mCherry::pio and $\mathrm{Np}^{P 6}$, mCherry::pio mutant embryos (Fig 22A; lanes 3-6). These fragments may correspond to full-length $\sim 115$ 


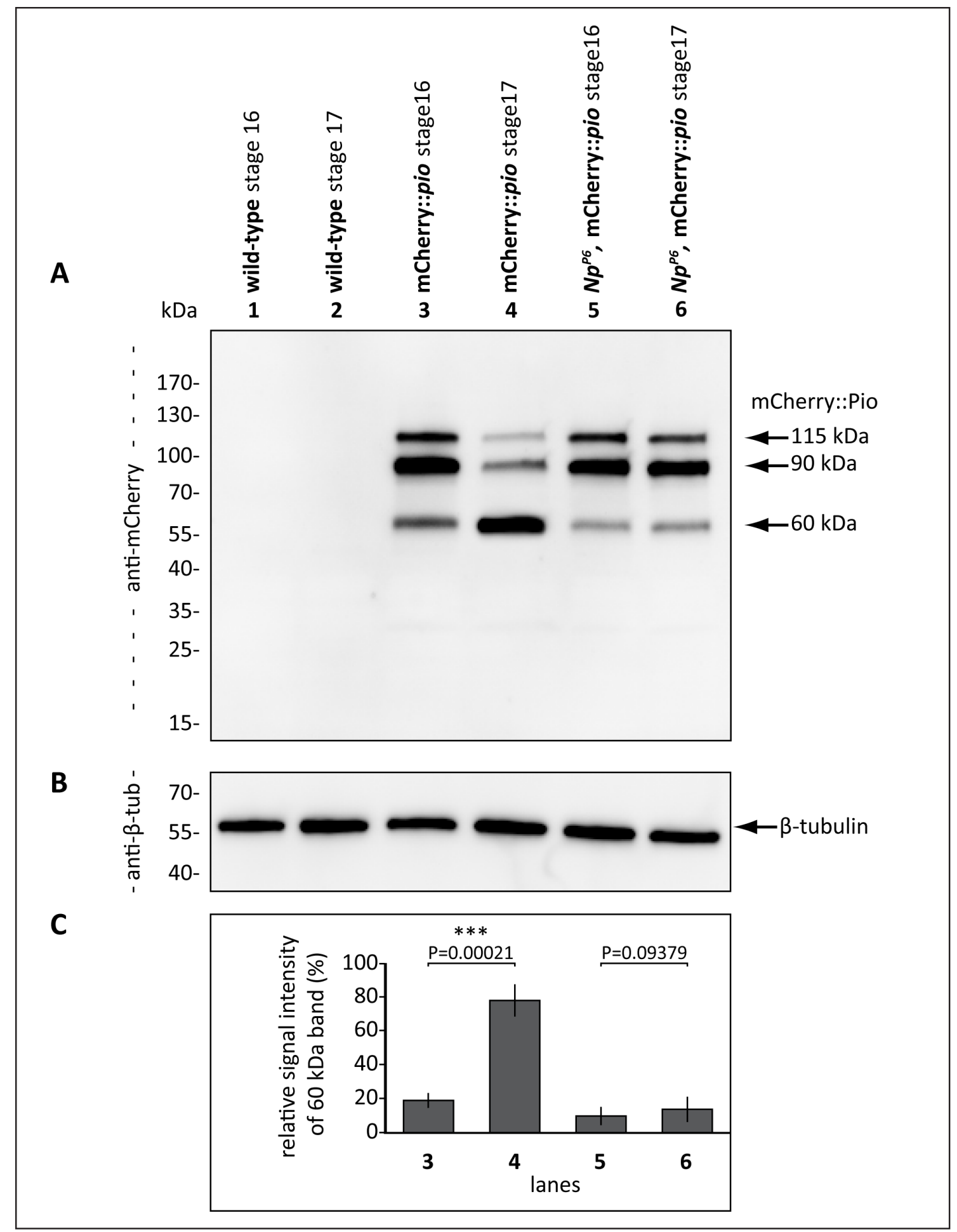

Figure 22: Notopleural mediates proteolytic processing of mCherry::Pio during apical extracellular matrix maturation

(A-B) Lysates of mid-stage 16 and mid-stage 17 wild-type (lanes 1-2), mCherry::pio/ $m$ Cherry::pio (lanes 3-4) and $N p^{P 6}$, mCherry::pio/ $N p^{P 6}$, $m$ Cherry::pio (lanes 5-6) embryos were analyzed by SDS-PAGE followed by western blotting with anti-mCherry (A) and antiB-tubulin (B) antibodies. For each lane, lysate of 18 embryos was loaded. Staining with anti-mCherry antibodies shows three immuno-reactive mCherry::Pio specific fragments of $\sim 115 \mathrm{kDa}, \sim 90 \mathrm{kDa}$ and $\sim 60 \mathrm{kDa}$ (A; lanes 3-6). Abundance of the $\sim 60 \mathrm{kDa}$ fragment is 
highly increased in lysates from mid-stage 17 mCherry::pio embryos (A; lane 4, compare to lane 3), indicating increased cleavage of mCherry::Pio. This stage-specific increase in $\sim 60$ $\mathrm{kDa}$ fragment level is not visible in lysates from $N p$ mutant embryos (A; lanes 5-6). Staining with anti-B-tubulin antibodies shows that the same amount of lysate was loaded in each lane (B). (C) Percentage of $\sim 60 \mathrm{kDa}$ fragment signal intensity of the total signal intensity of all three mCherry::Pio specific fragments is shown for lanes 3-6. Data represent mean values of two independent experiments. Error bars indicate standard deviation and statistical significance was calculated with two-tailed t-tests.

kDa mCherry::Pio (predicted: $78 \mathrm{kDa}$ ), $\sim 90 \mathrm{kDa}$ Furin-cleaved mCherry::Pio (predicted: $67 \mathrm{kDa}$; see Fig 18D for position of predicted Furin cleavage site) and a $\sim 60 \mathrm{kDa}$ mCherry::Pio fragment that was cleaved at an unknown site. The discrepancies between mass of observed Pio fragments and predicted mass was also observed in other studies and might be due to post-translational modifications (Bökel et al. 2005). While all three fragments are detectable in lysates of mid-stage 16 and mid-stage 17 mCherry::pio embryos, the $\sim 60 \mathrm{kDa}$ mCherry::Pio fragment is present at higher levels in lysates of mid-stage 17 embryos (Fig 22A; compare lanes 3 and 4). Furthermore, mCherry::Pio fragments of $\sim 115 \mathrm{kDa}$ and $\sim 90 \mathrm{kDa}$ are present at lower levels in lysates from mid-stage 17 mCherry::pio embryos (Fig 22A; compare lanes 3 and 4). These stage-specific differences in abundance of the mCherry::Pio fragments are not visible in lysates from $N p$ mutant embryos, i.e. level of the $\sim 60 \mathrm{kDa}$ mCherry::Pio fragment is not increased in lysates from midstage $17 \mathrm{~Np}^{P 6}$, mCherry::pio mutant embryos (Fig 22A; compare lanes 5 and 6).

To quantify the relative abundance of the $\sim 60 \mathrm{kDa}$ mCherry::Pio fragment, percentage of signal intensity of the $\sim 60 \mathrm{kDa}$ fragment from the total signal intensity of all three mCherry::Pio fragments was calculated for each lane. Abundance of the $\sim 60 \mathrm{kDa}$ fragment in lysates of mCherry::pio embryos increases from $\sim 20 \%$ in mid-stage 16 to $\sim 80 \%$ in mid-stage 17 (Fig 22C). In contrast, the $\sim 60 \mathrm{kDa}$ fragment shows an invariable portion of approximately $10 \%$ in lysates of $N p$ mutant embryos (Fig 22C). These experiments indicate that proteolytic processing of Pio during aECM maturation in stage 17 is mediated by $\mathrm{Np}$. 


\subsubsection{Non-cell autonomous function of Notopleural during embryonic tracheal development}

In the tracheal system, Dpy and $\mathrm{Np}$ predominately localize to the intraluminal extracellular space (chapters 2.1 .3 and 2.1.8). Also, Pio localizes to the tracheal apical cell membranes and intraluminal space (see chapter 2.1.10). Therefore, Np may interact with Pio and Dpy in the intraluminal space, raising the possibility that Np might mediate tracheal aECM maturation by a non-cell autonomous mechanism. To test this hypothesis, an UAS-Np::GFP responder line was established and expression of UAS- $N p:$ GFP was driven by $A b d-B$-Gal4 in $N p$ mutant embryos. The $A b d-B$-Gal4 driver is expressed in ectodermal tissues of the posterior embryonic parasegments A8-A14 and therefore, expression of Np::GFP in the tracheal system is only activated in these segments. $N p$ mutant embryos with $A b d-B$-Gal4 mediated Np::GFP expression were stained for GFP and chitin to visualize Np::GFP localization and aECM maturation in the tracheal system.

In stage 16 embryos, localization of $\mathrm{Np}:$ GFP is restricted to parasegments A8-A14 ( $A b d-B$ expression domain; Fig 23A-A"). While the intraluminal chitin cable forms normally throughout the whole tracheal system, intraluminal Np::GFP (arrow in Fig 23B") is only present in segments with Np::GFP expression and not detectable apart from the $A b d-B$ expression domain (Fig 23B-B"). This spatial pattern of Np::GFP localization is changed in early-stage 17, coinciding with tracheal aECM maturation and intraluminal chitin cable degradation. In early-stage 17 embryos, Np::GFP is detectable throughout the whole tracheal system, unrestricted by the $A b d-B$ expression domain (Fig 23C-C"). Both, intraluminal (arrow in Fig 23D") and apical membrane-associated Np::GFP (arrowhead in Fig 23D") are present in the entire tracheal system (Fig 23D-D"), similar to what has been observed for endogenously expressed Np::GFP (Fig 8E-F"). The diffused Np::GFP mediates chitin cable contraction during degradation (Fig 23D-D') as seen previously in wildtype embryos (Fig 15B' and 16B'). Furthermore, late-stage 17 embryos show wildtype-like organization of chitin strands in the tracheal taenidial folds throughout the whole tracheal system (Fig 23E) and tracheal gas filling (Fig 23G). These observations indicate that $\mathrm{Np}$ mediates tracheal aECM maturation and gas filling in a non-cell autonomous manner. 


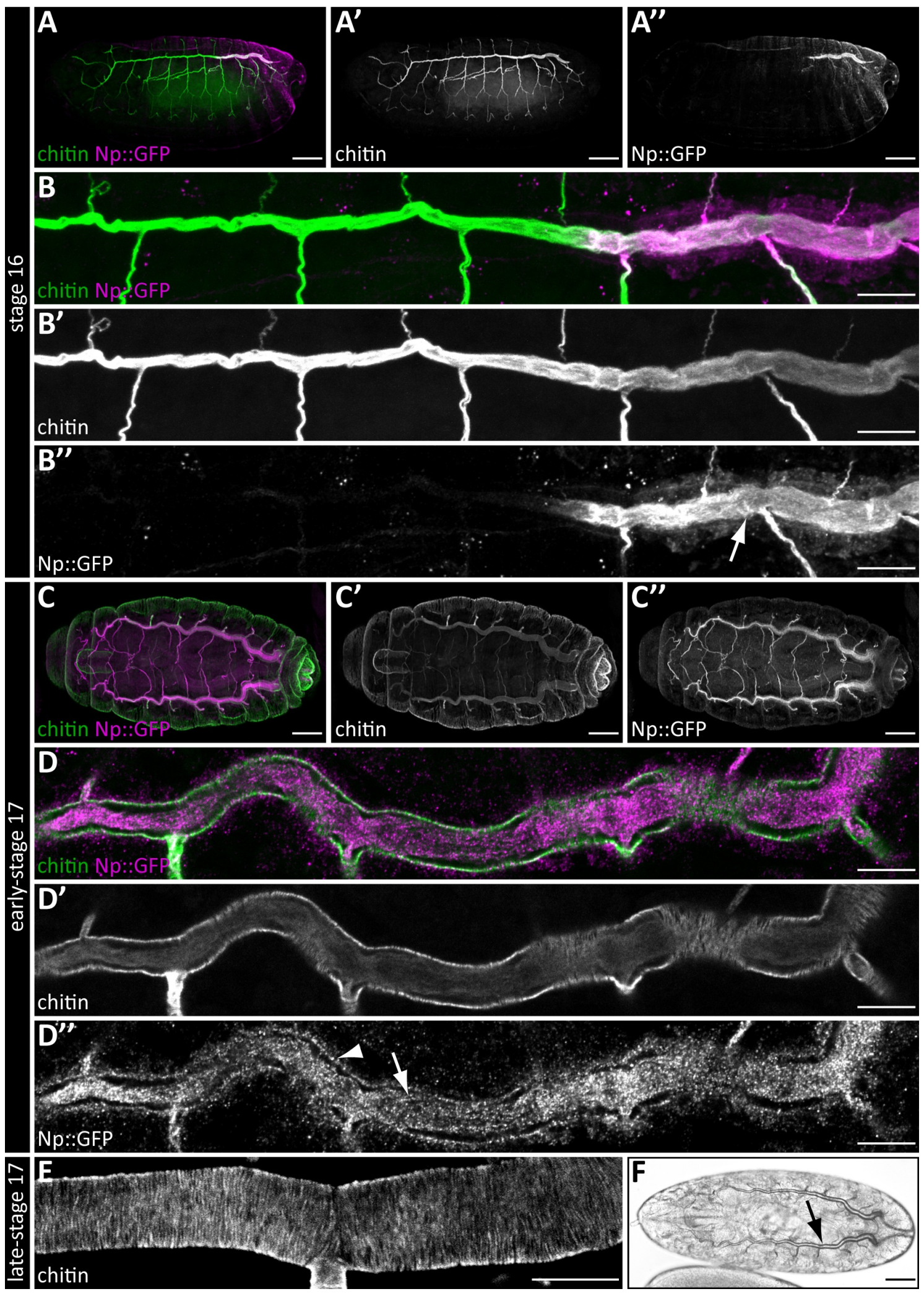

Figure 23: Non-cell autonomous function of Notopleural in the tracheal system

(A-E) Confocal LSM images of $N p^{P 6}, \mathrm{UAS}-N p:: \mathrm{GFP} / N p^{C 2} ; A b d-B$-Gal4/+ embryos (A-A", C-C") and their tracheal dorsal trunks (B-B", D-E) stained with FITC-labeled CBP (chitin) and anti-GFP antibodies. Late-stage 17 dorsal trunk (E) is shown as a z-stack projection. In stage 16 embryos, Np::GFP is only present in the $A b d-B$ expression domain (A-B") and localizes to the tracheal intraluminal space (arrow in $B "$ ). Np::GFP is present throughout 
the whole tracheal system of early-stage 17 embryos (C-D") and localizes to the intraluminal space (arrow in D") and to the apical cell membranes (arrowhead in D"). The diffused Np::GFP mediates wild-type-like tracheal aECM maturation, visible by chitin cable contraction during early-stage 17 (D-D') and normal taenidial folds formation (E; compare to Fig 13C). (F) Bright field light microscopic image of a late-stage $17 \mathrm{~Np}^{P 6}$, UAS$N p:: \mathrm{GFP} / N p^{c 2} ; A b d-B$-Gal4/+ embryo with a gas filled tracheal system (arrow). Images of stage 16 embryo show a lateral view (A-B") while images of stage 17 embryos show a dorsal view (C-F). Scale bars correspond to $50 \mu \mathrm{m}$ (A-A", C-C", F) and $10 \mu \mathrm{m}$ (B-B", D-E), respectively.

\subsubsection{Function of Notopleural depends on its catalytic activity}

While previous results show that $\mathrm{Np}$ mediates proteolytic processing of Pio (chapter 2.1.12), it remained unclear to what extend $\mathrm{Np}$ function is dependent on its catalytic activity. To elucidate the role of $\mathrm{Np}$ catalytic activity, flies that endogenously express catalytically inactive Strep-tagged $\mathrm{Np}$ were generated. The $\Phi C 31$ integrase attP/attB system was used to insert a construct that encodes for $\mathrm{Np}$ with serine 990 of the catalytic triad substituted with alanine, and a Strep-tag fused to the carboxy-terminus ( $N p^{S 990 A}::$ Strep) into the previously established $N p^{\text {att } P}$ landing site (Fig 24A-C; Fig 7). It is well established that mutations of the serine in the catalytic triad disrupt activity of serine proteases (Polgár 2005). As a control, flies that endogenously express wild-type Strep-tagged $\mathrm{Np}$ ( $N p::$ Strep) were also generated with the $\Phi C 31$ integrase attP/attB system. Both, $N p:$ Strep and $N p^{S 990 A}::$ Strep embryos were then analyzed by whole-mount stainings of chitin using FITC-labeled CBP, by in vivo observations and by cuticle preparations. Homozygous $N p:$ :Strep embryos show wild-type-like taenidial folds organization (Fig 24D), gas filling of the tracheal system (arrow in Fig 24F) and wild-type-like formation of epidermal cuticle denticle belts (Fig 24H). In contrast, embryos that are homozygous for $N p^{S 990 A:: S t r e p ~ d i s p l a y ~ a ~ m i s o r g a n i z e d ~ m a t u r e ~ t r a c h e a l ~ a E C M ~}$ with randomly distributed bulges (Fig 24E; arrowheads) and lack tracheal gas filling (arrow in Fig 24G). Furthermore, $N p^{S 990 A}:$ Strep embryos lack formation of denticles in the epidermal cuticle (Fig 24I) and do not hatch. Thus, $N p^{5990 A}::$ Strep embryos display phenotypes that were described previously for $N p$ loss-of-function mutants (chapters 2.1.2, 2.1.6 and 2.1.7), indicating that $\mathrm{Np}$ mediates aECM maturation and tracheal gas filling by its catalytic activity. 


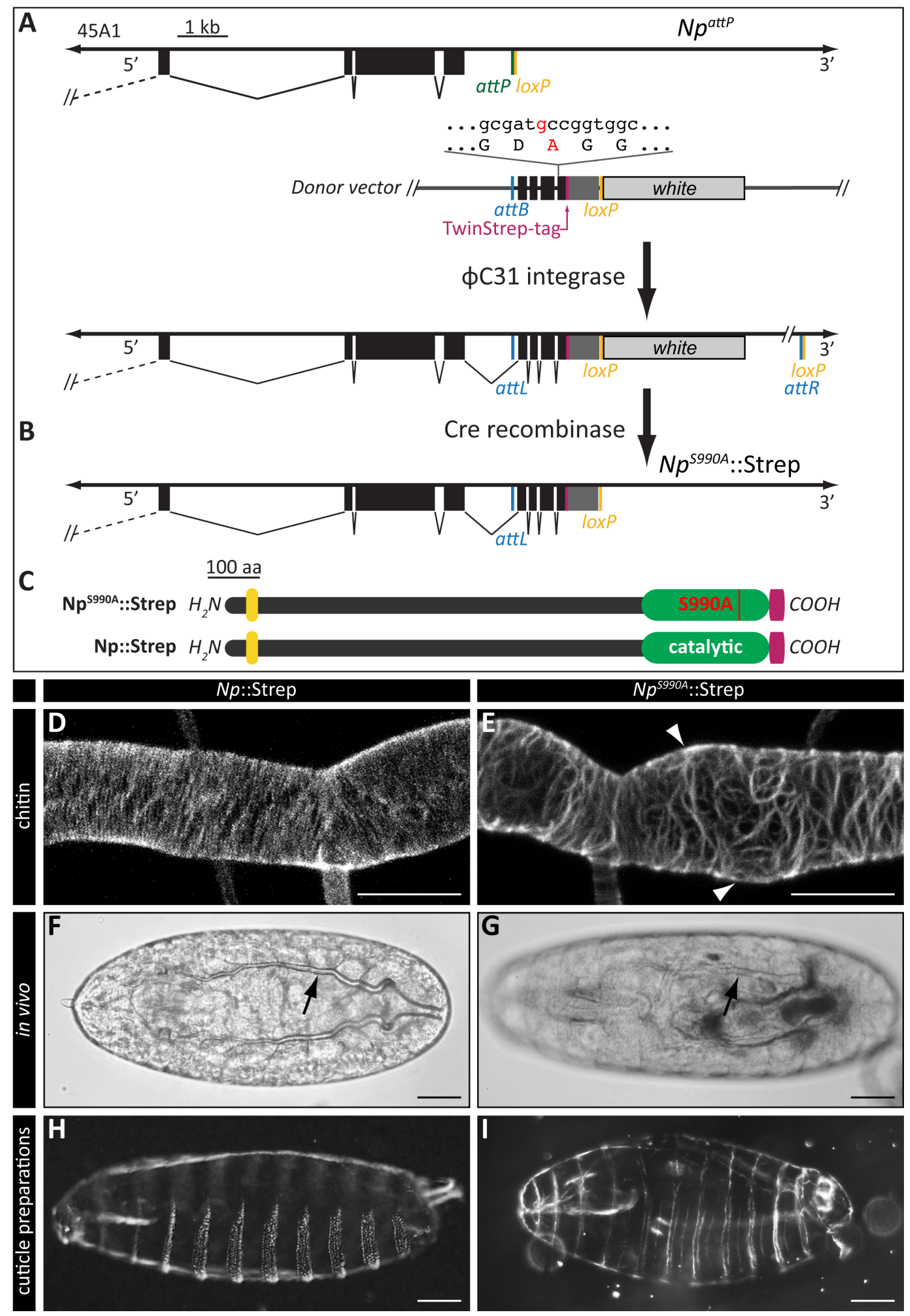

Figure 24: Notopleural mediates apical extracellular matrix maturation and tracheal gas filling by its catalytic activity

(A-C) Schema of generation of flies that endogenously express Np::Strep or catalytically

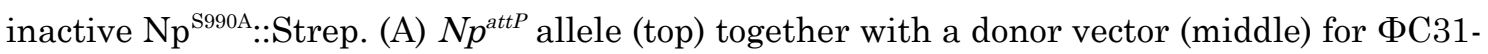


integrase-mediated knock-in of $N p$ genomic region with a TwinStrep-tag encoding sequence (magenta) integrated 3 ' to the final protein coding exon, generating white ${ }^{+}$flies encoding Strep-tagged Np (bottom). A single nucleotide mutation in exon 10 (t to g; red letters) generates substitution of serine 990 with alanine. (B) $N p^{S 990 A}::$ Strep allele after Cre recombinase-mediated white gene excision. Black boxes indicate protein coding exons and

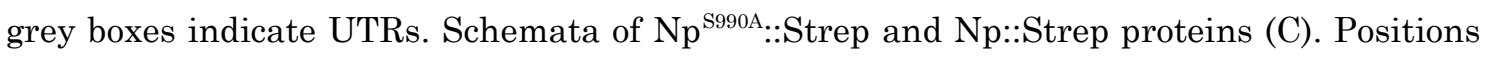
of transmembrane domains (yellow), catalytic domains (green) and TwinStrep-tags (magenta) are shown. (D-E) Confocal LSM z-stack projection images of tracheal dorsal trunks of mid-stage $17 N p::$ Strep (D) and $N p^{S 990 A}::$ Strep (E) embryos that were stained with FITC-labeled CBP (chitin). Np::Strep embryos show wild-type-like organization of chitin strands (D; compare with Fig 13C) while $N p^{S 990 A}::$ Strep embryos display misorganized chitin strands and formation of bulges (arrowheads) as observed in $N p$ mutant embryos (E; compare with Fig 13D and Fig 16F"). Scale bars correspond to $10 \mu \mathrm{m}$. (F-G) Bright field light microscopic images of late-stage $17 N p::$ Strep (F) and $N p^{S 990 A}::$ Strep (G) embryos shown from dorsal. $N p$ ::Strep embryos show gas filling of the tracheal system (arrow in F), while $N p^{S 990 A}:$ :Strep embryos lack tracheal gas filling (arrow in G). Scale bars correspond to $50 \mu \mathrm{m}$. (H-I) Dark field light microscopic images of cuticle preparations of late-stage 17 $N p::$ Strep $(\mathrm{H})$ and $N p^{\text {S990A }:: S t r e p ~(I) ~ e m b r y o s ~ s h o w n ~ f r o m ~ l a t e r a l . ~} N p::$ Strep embryos develop wild-type-like denticle belts (H; compare with Fig 14A), while $N p^{\text {S990A }:: S t r e p ~ e m b r y o s ~}$ display only rudimentary denticles as seen in $N p$ mutant embryos (I; compare with Fig 14C). Scale bars correspond to $50 \mu \mathrm{m}$.

To analyze whether $\mathrm{Np}^{\mathrm{S} 990 \mathrm{~A}}::$ Strep localizes normally in the tracheal system despite its catalytic inactivity, stainings of $N p::$ Strep and $N p^{S 990 A}::$ Strep embryos with CBP and anti-Strep antibodies were performed. In mid-stage 16 and mid-stage 17 embryos, Np::Strep localizes to the tracheal intraluminal aECM and to the apical surface of tracheal cells (arrows and arrowheads; Fig 25A-B"). This localization pattern is identical with previously shown localization of Np::GFP (Fig 8F-F").

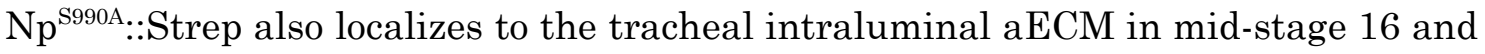
mid-stage 17 embryos (arrows; Fig 25C-D"). However, apical membrane-associated localization of $\mathrm{Np}^{\mathrm{S990A}}:$ :Strep is not clearly distinguishable. Furthermore, while Np::Strep shows well-defined co-localization with chitin of the mature tracheal

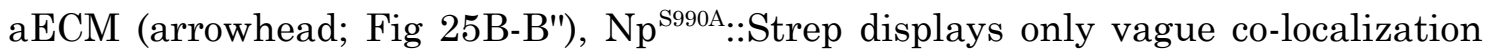
with the misorganized chitin strands of mid-stage 17 trachea and is also present on the cytoplasmic side of chitin (yellow arrowhead; Fig 25D-D"). These observation show that apical membrane associated localization of $\mathrm{Np}$ depends on its catalytic activity. 

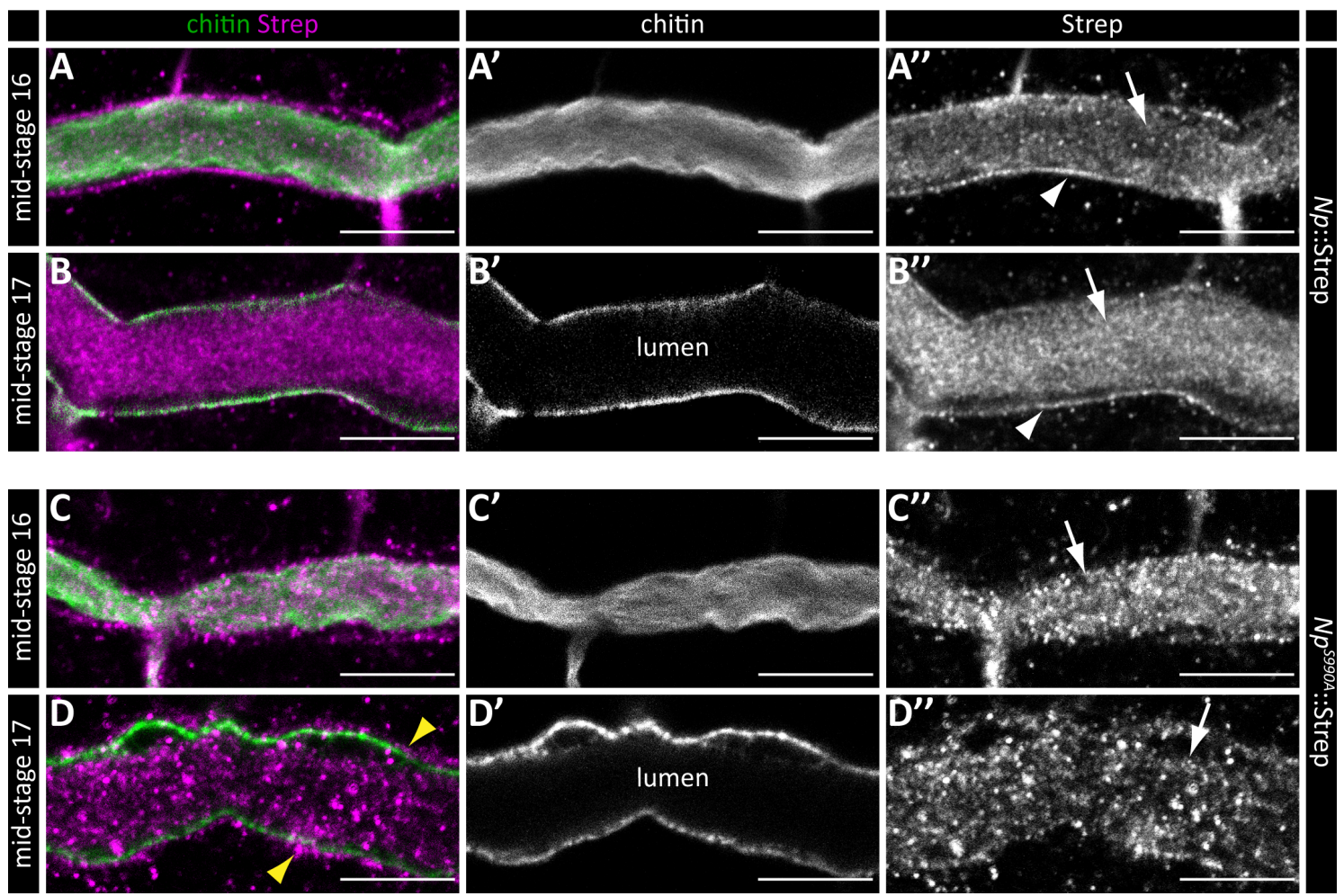

Figure 25: Association of Notopleural to tracheal apical cell membranes depends on its catalytic activity

Confocal LSM images of dorsal trunks of mid-stage 16 (A-A", C-C") and mid-stage 17 (B-B", D-D") $N p::$ Strep (A-B") and $N p^{S 990 A:: S t r e p ~(C-D ") ~ e m b r y o s ~ t h a t ~ w e r e ~ s t a i n e d ~ w i t h ~ F I T C-~}$ labeled CBP (chitin) and anti-Strep antibodies (Strep). Np::Strep is detectable in the intraluminal space (arrows in A", B") and at the apical surface of tracheal cells (arrowheads

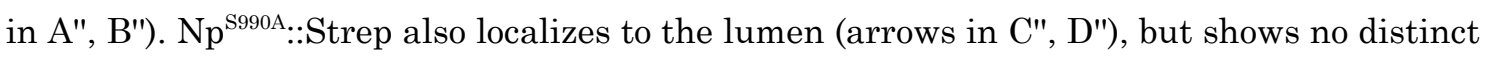
apical membrane-associated localization (C-D"). In mid-stage 17 embryos, $\mathrm{Np}^{\text {S990A }:: S t r e p}$ is present on the cytoplasmic side of the chitin strands (yellow arrowheads in D). Similar localization is not detectable for Np::Strep (B-B"). Scale bars correspond to $10 \mu \mathrm{m}$.

\subsubsection{Notopleural zymogen activation is an autocatalytic mechanism}

Several vertebrate TTSPs including matriptase, are known to be capable of autocatalytic activation. In this mechanism, cleavage of a TTSP zymogen is mediated by its intrinsic catalytic activity (Lee et al. 2007; Qiu et al. 2007). Most observations of TTSP autoactivations were done in vitro or in cultured cells and whether this mechanism is relevant in vivo and how it is regulated is an ongoing debate (Antalis et al. 2011). To test if $\mathrm{Np}$ zymogen activation in the $D$. melanogaster embryo is autocatalytic and therefore depends on its own catalytic activity, zymogen cleavage of $\mathrm{Np}:$ :Strep and catalytically inactive $\mathrm{Np}^{\text {S990A.:Strep }}$ were analyzed. Lysates of homozygous and heterozygous late-stage $16 \mathrm{~Np}:$ Strep

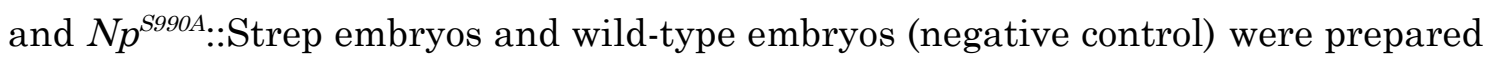




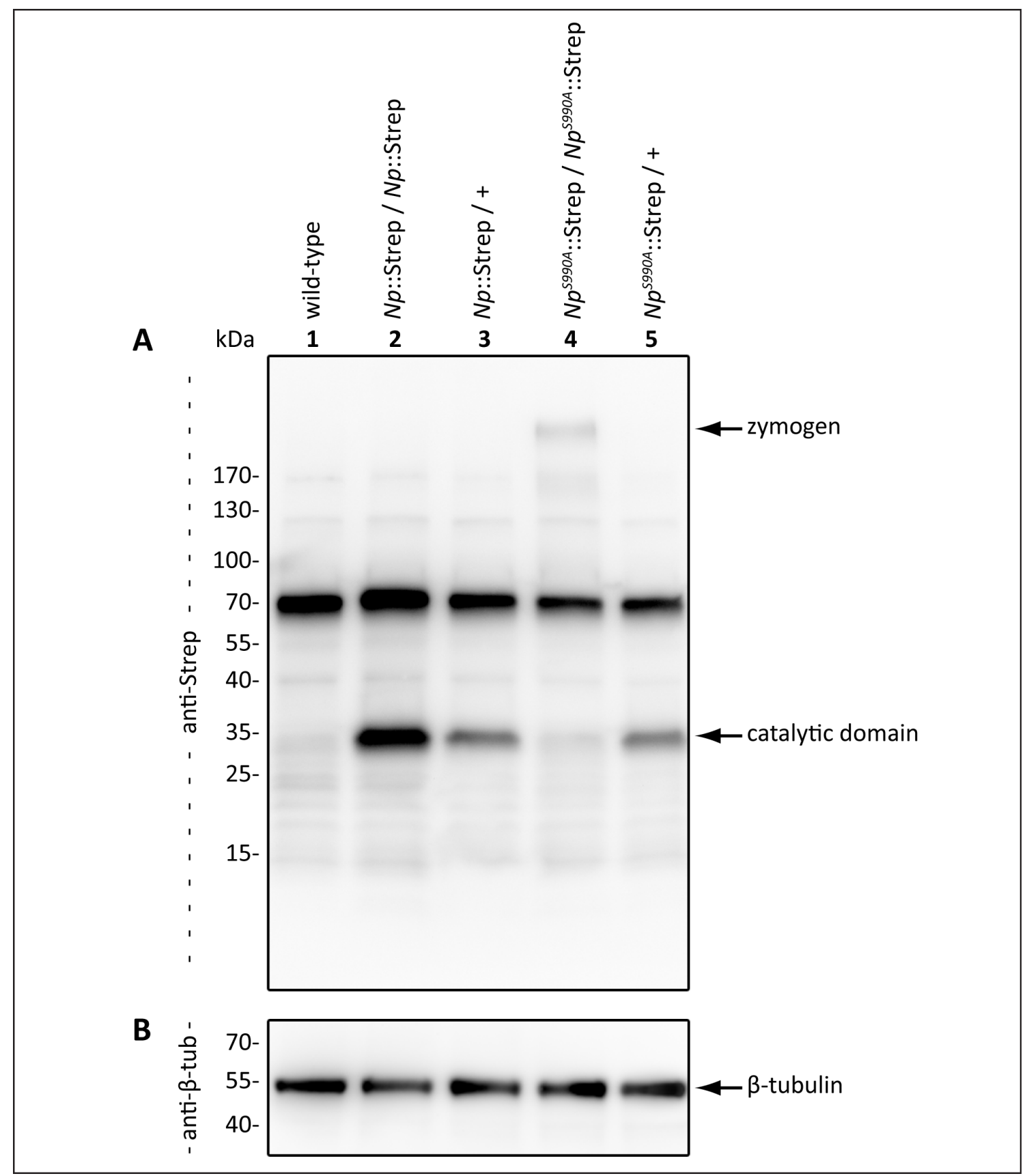

\section{Figure 26: Cleavage of the Notopleural zymogen depends on its own catalytic activity}

Lysates of late-stage 16 wild-type (lane 1), homozygous (lane 2) and heterozygous (lane 3) $N p::$ Strep embryos, and homozygous (lane 4) and heterozygous (lane 5) $N p^{S 990 A:: \text { Strep }}$ embryos were analyzed by SDS-PAGE, subsequent western blotting and stainings with anti-Strep (A) and anti-B-tubulin (B) antibodies. Lysates were prepared under denaturing and reducing conditions and lysate from 30 embryos was loaded for each lane. Staining with anti-Strep antibodies shows an unspecific band of $\sim 70 \mathrm{kDa}$ and $\mathrm{Np}$ specific bands of $\sim 35 \mathrm{kDa}$ and $\sim 230 \mathrm{kDa}$ that presumably correspond to the $\mathrm{Np}$ catalytic domain and zymogen, respectively (A). In lysate from homozygous $N p^{S 990 A}:$ :Strep embryos (lane 4 in A), only the $\mathrm{Np}$ zymogen is detectable, indicating that activation of $\mathrm{Np}$ depends on its own catalytic activity. Staining with anti-B-tubulin antibodies shows that the same amount of lysate was loaded in each lane (B). 
and analyzed by SDS-PAGE followed by western blotting and immuno-stainings with anti-Strep (Fig 26A) and anti-B-tubulin (Fig 26B) antibodies.

Staining with anti-Strep antibodies shows a band of $\sim 70 \mathrm{kDa}$ in all lanes, which is due to unspecific binding of the antibodies since this band is also present in lysate of wild-type embryos (lane 1; Fig 26A). In addition, a fragment of $\sim 35 \mathrm{kDa}$ is present in lysates of homozygous and heterozygous $N p:$ Strep embryos (lanes 2-3) as well as in lysates of heterozygous $N p^{\text {S990A }:: S t r e p ~ e m b r y o s ~(l a n e ~} 5$ in Fig 26A). These fragments match the predicted mass of the Strep-tagged Np catalytic domain $(32 \mathrm{kDa}$ ) and therefore indicate cleavage of the $\mathrm{Np}$ zymogen. In lysate from homozygous $N p^{S 990 A}::$ Strep embryos, no cleaved $\sim 35 \mathrm{kDa} \mathrm{Np}$ catalytic domain is detectable and instead, a fragment of $\sim 230 \mathrm{kDa}$ is present (lane 4 in Fig 26A). This fragment presumably corresponds to the $\mathrm{Np}$ zymogen. While full-length Streptagged $\mathrm{Np}$ has a predicted mass of $114 \mathrm{kDa}$, the observed mass of $\sim 230 \mathrm{kDa}$ might be due to post-translational modifications. Indeed, an in silico analysis using NetOGlyc 4.0 algorithm predicts 281 O-glycosylation sites in $\mathrm{Np}$ (Steentoft et al. 2013). While no cleavage of catalytically inactive $\mathrm{Np}^{\text {S990A }:: S t r e p}$ is observed in lysate from homozygous $N p^{S 990 A}::$ Strep embryos (lane 4 in Fig 26A ), the cleaved catalytic domain of $\mathrm{Np}^{\mathrm{S} 990 \mathrm{~A}}:$ :Strep is detectable in lysate from embryos that carry a wild-type allele of $\mathrm{Np}$ heterozygously (lane 5 in Fig 26A). These experiments suggest that proteolytic activation of the $\mathrm{Np}$ zymogen in $D$. melanogaster embryos depends on its own catalytic activity and therefore may represent autocatalytic activation.

\subsubsection{Human matriptase is functionally homologous to Notopleural}

As shown in chapters 2.1.2 - 2.1.11, D. melanogaster $\mathrm{Np}$ is essential for embryonic aECM maturation, aECM - cell interactions, tracheal gas filling and tracheal transepithelial barrier function maintenance. Furthermore, Np zymogen activation is an autocatalytic mechanism (chapter 2.1.15). Similarly, vertebrate matriptase is known to regulate ECM remodeling and degradation, ECM - cell interactions and maintenance of the transepithelial barrier in various tissues (Miller and List 2013; Milner et al. 2010). Also, the matriptase zymogen is activated by an autocatalytic mechanism (Lee et al. 2007). Besides these functional similarities, D. melanogaster $\mathrm{Np}$ and human matriptase share a $41 \%$ sequence identity in their catalytic domains (Fig 4). To test whether these striking similarities are based on a 
functional homology of matriptase and $\mathrm{Np}$, human matriptase was expressed in the tracheal system of $N p$ mutant embryos. For these experiments, UAS-matriptase responder lines were established. In addition, UAS- $N p^{S 990 A}$ (catalytically inactive $\mathrm{Np}$ ) and UAS-lint responder lines were established as controls. While previous experiments already showed that endogenously expressed $N p^{S 990 A}::$ Strep does not rescue the $N p$ mutant phenotype (chapter 2.1.14), the UAS- $N p^{S 990 A}$ responder line was established to rule out any artifacts by elevated expression levels due to the UAS/Gal4 system. The UAS-lint responder line was established to determine the specificity of rescue effects. Chapter 2.3 shows that lint encodes a TTSP with high sequence similarity to $\mathrm{Np}$ and that lint mutant embryos display similar phenotypes as $N p$ mutant embryos.

To analyze whether human matriptase specifically rescues the $N p$ mutant tracheal phenotype, whole-mount stainings of chitin in $N p$ mutant embryos with pantracheal expression of either $\mathrm{Np}:$ GFP, $\mathrm{Np}^{\mathrm{S} 990 \mathrm{~A}}$, matriptase or Lint were performed. Strikingly, tracheal expression of human matriptase rescues the $N p$ mutant tracheal aECM phenotype (Fig 27D), as observed for tracheal expression of Np::GFP (Fig 27B). In both cases, chitin strands of the mature tracheal aECM show a parallel evenly spaced organization (Fig 27B, D) and diameters and frequencies of chitin strands are wild-type-like (Fig 27F). In contrast, organization of mature aECM chitin strands of $N p$ mutant embryos with tracheal expression of $\mathrm{Np}^{\mathrm{S} 990 \mathrm{~A}}$ or Lint is disrupted (Fig $27 \mathrm{C}, \mathrm{E}$ ) and resembles the $N p$ mutant phenotype (Fig 27A). Indeed, diameters and frequencies of chitin strands in trachea of these embryos are very similar to $N p$ mutant trachea (Fig $27 \mathrm{~F}$ ).

These findings indicate that human matriptase substitutes for the function of $\mathrm{Np}$ during tracheal aECM maturation in D. melanogaster. However, while tracheal expression of $\mathrm{Np}$ (or $\mathrm{Np}: \mathrm{GFP}$ ) in $N p$ mutant embryos leads to wild-type-like gasfilling of the tracheal system (Fig 5E; Fig 23F), $N p$ mutant embryos with tracheal expression of matriptase lack tracheal gas filling. Furthermore, rescue of the mature tracheal aECM organization was not observed in all $N p$ mutant embryos with tracheal expression of matriptase. This incomplete rescue of the $N p$ mutant phenotype by matriptase may be caused by aberrant localization of matriptase, e.g lack of secretion into the tracheal lumen. Therefore, subcellular localization of matriptase in the tracheal system of $N p$ mutant embryos was analyzed. In 


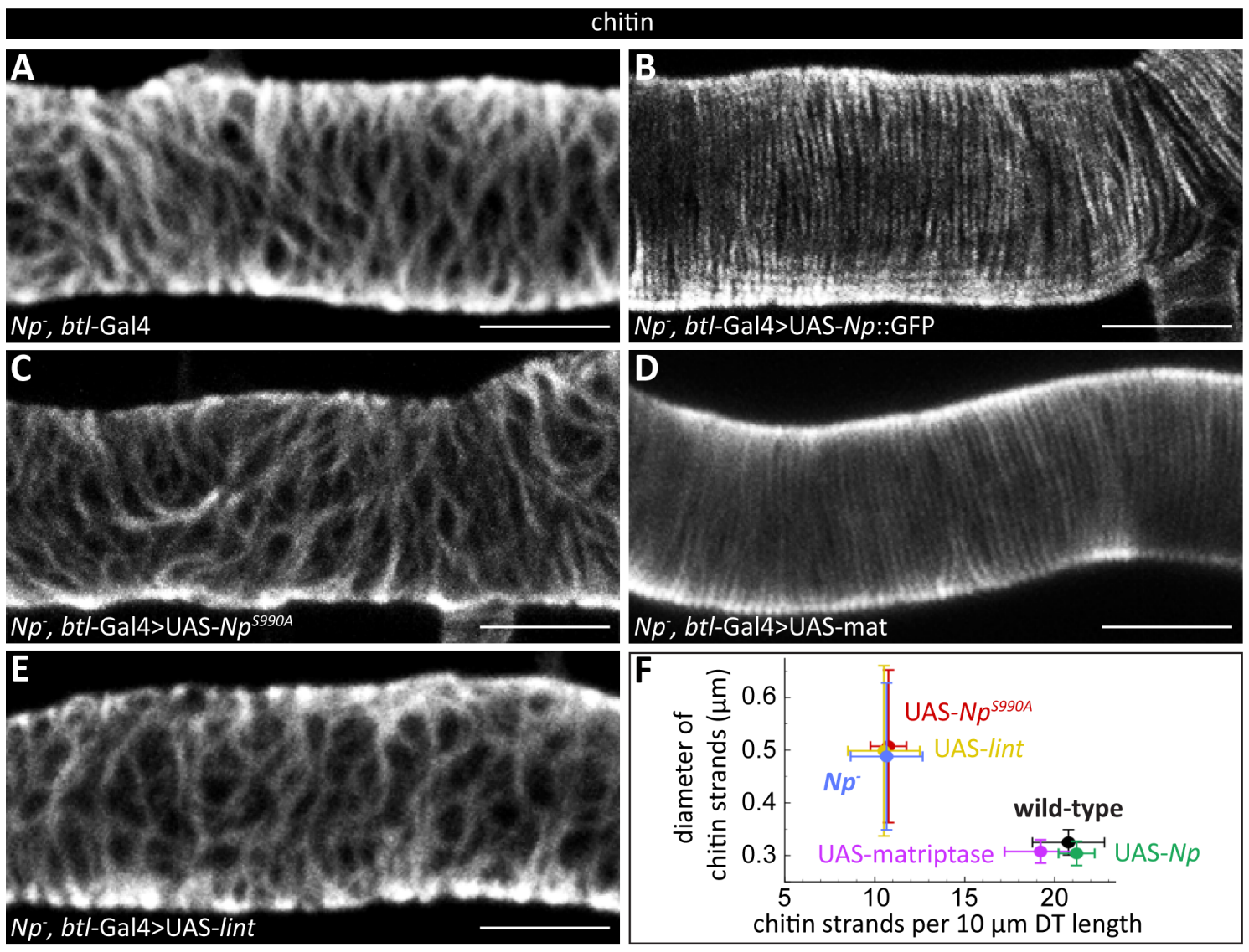

Figure 27: Expression of human matriptase rescues the Notopleural mutant tracheal apical extracellular matrix phenotype

(A-E) Confocal LSM z-stack projection images of dorsal trunks of stage $17 \mathrm{~Np} p^{C 2}$, btl-Gal4 / $N p^{P 6}(\mathrm{~A}), N p^{C 2}, b t l$-Gal4 / $N p^{P 6}$; UAS- $N p::$ GFP (B), $N p^{C 2}$, btl-Gal4 / $N p^{P 6}$; UAS- $N p^{\mathrm{S} 990 \mathrm{~A}}$ (C), $N p^{C 2}$, btl-Gal4 / $N p^{P 6}$; UAS-matriptase (D), and UAS-lint; $N p^{C 2}$, btl-Gal4 / $N p^{P 6}$ (E) embryos that were stained with FITC-labeled CBP (chitin). $N p$ mutant embryos with tracheal expression of Np::GFP (B) or human matriptase (D) show wild-type-like taenidial folds organization while tracheal expression of $\mathrm{Np}^{5990 \mathrm{~A}}$ (C) or Lint (E) can not rescue the $N p$ mutant phenotype (A). Scale bars correspond to $5 \mu \mathrm{m}$. (F) Quantification of diameter and frequency of stage 17 dorsal trunk chitin strands. Data represent mean values of measurements in 10 embryos for each genotype and error bars indicate standard deviations. Data for $N p$ mutant embryos (blue) and wild-type embryos (black) were already shown in Figure 13E. They are presented here again for comparison with data from $\mathrm{Np}$ mutant embryos with tracheal expression of $\mathrm{Np}: \mathrm{GFP}$ (green), $\mathrm{Np}^{\mathrm{S} 990 \mathrm{~A}}$ (red), human matriptase (magenta) or Lint (yellow). For method details see chapter 4.

addition, rescue of the $N p$ mutant phenotype by matriptase was quantified regarding tracheal aECM organization and gas filling.

Ectopically expressed Np::GFP in the trachea of $N p$ mutant embryos exclusively localizes to the tracheal lumen in stage 16 (Fig 28A-A"), similar to what has been observed for endogenously expressed Np::GFP (Fig 8E-E”). In contrast, human 
matriptase expressed in the tracheal system of $N p$ mutant embryos localizes to the cytoplasm and membranes of tracheal cells and is not detectable in the tracheal lumen (Fig 28B-B"). Lack of matriptase secretion into the tracheal lumen is attended by incomplete rescue of the $N p$ mutant phenotype. Only $\sim 55 \%$ of $N p$ mutant embryos with tracheal matriptase expression show wild-type-like organization of tracheal aECM chitin strands throughout the entire tracheal system and all of these embryos lack tracheal gas filling (Fig 28E). Unlike expression of matriptase, ectopic tracheal expression of $\mathrm{Np}$ in $\mathrm{Np}$ mutant embryos rescues aECM chitin strands organization in $\sim 95 \%$ of the embryos and restores tracheal gas filling in $\sim 90 \%$ of the embryos (Fig 28E). However, observed rescue of the $N p$ mutant phenotype by matriptase is specific to matriptase function since tracheal expression of $\mathrm{Np}^{\mathrm{S} 990 \mathrm{~A}}$ or Lint can not rescue the $N p$ mutant phenotype (Fig $28 \mathrm{E})$.

Due to the discrepancy between Np::GFP localization to the tracheal lumen and matriptase localization to tracheal cells (Fig 28A-B"), it seemed likely that mediation of matriptase secretion into the tracheal lumen might increase its capability to rescue the $N p$ mutant phenotype. Several factors are implicated to contribute to matriptase secretion and ectodomain shedding (Miller and List 2013; Tsuzuki et al. 2010). Two essential regulators of matriptase activity and localization are hepatocyte growth factor activator inhibitor (HAI) -1 and HAI-2. HAI-1 affects matriptase zymogen activation and is suggested to regulate matriptase activity in the extracellular environment (Miller and List 2013; Chen et al. 2010). HAI-2 inhibits matriptase activity in the intracellular milieu and mediates translocation of matriptase to the cellular membrane and regulates subsequent ectodomain shedding (Nonboe et al. 2017; Wu et al. 2017; Friis et al. 2014). To test whether these co-factors might induce matriptase secretion into the tracheal lumen of $D$. melanogaster embryos, UAS responder strains for human HAI-1 and HAI-2 were established and used for trachea-specific expression. Coexpression of human matriptase and HAI-1 in the tracheal system of $N p$ mutant embryos results in intracellular and membrane-associated localization of matriptase (Fig 28C-C"), as observed for matriptase in the absence of HAI-1 (Fig 28B-B"). Tracheal co-expression of matriptase and HAI-1 is also not efficient in rescuing the $N p$ mutant phenotype, since chitin strands organization is rescued in only $\sim 28 \%$ of these embryos (Fig 28E). Strikingly, tracheal co-expression of human 


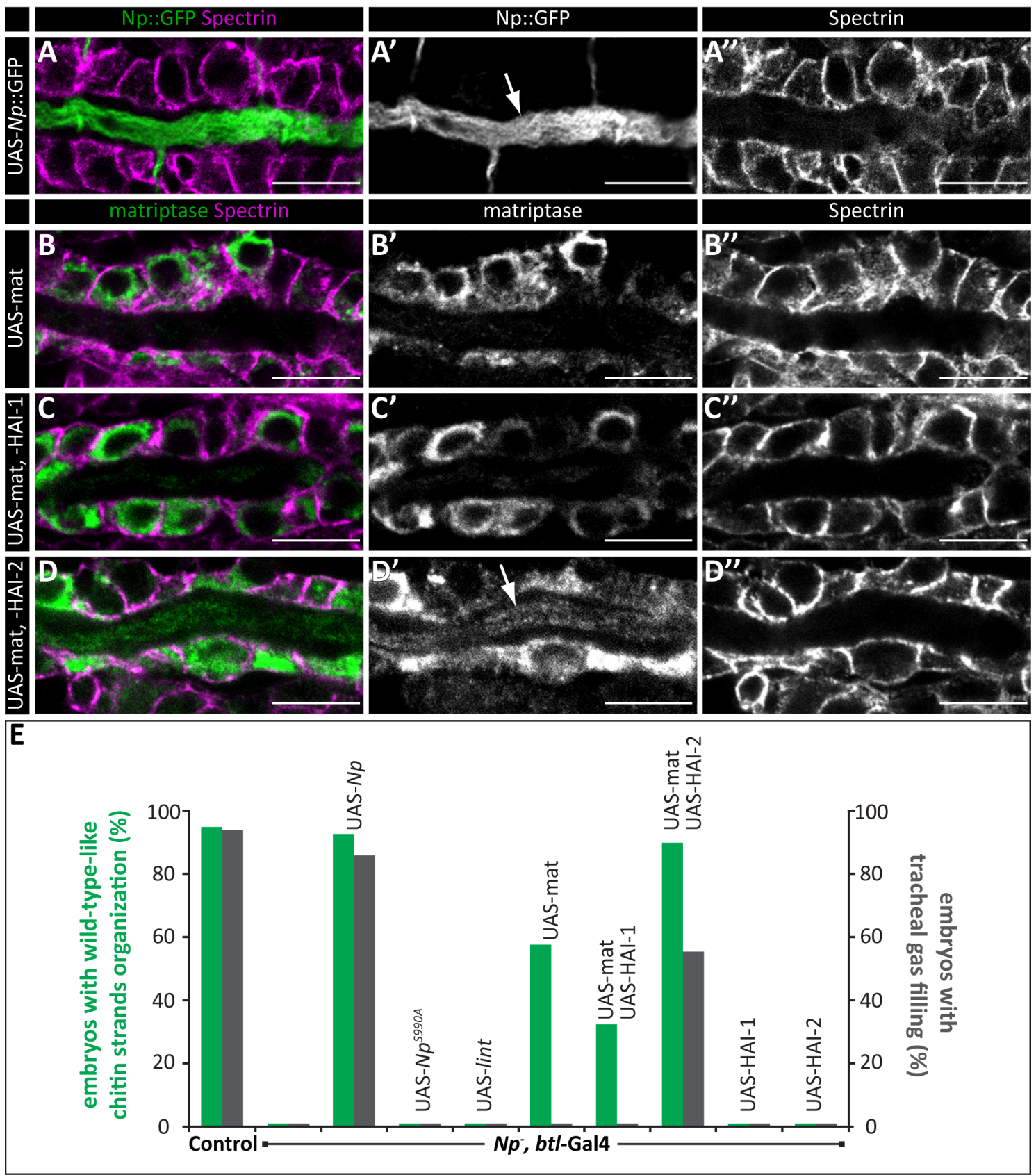

Figure 28: Secretion of human matriptase into the tracheal lumen is mediated by HAI-2

(A-D“) Confocal LSM images of tracheal dorsal trunks of stage $16 \mathrm{~Np}^{C 2}$, btl-Gal4 / $\mathrm{Np}^{P 6}$, UAS- $N p:$ :GFP (A-A“), $N p^{C 2}$, btl-Gal4 / $N p^{P 6}$, UAS-matriptase (B-B“), $N p^{C 2}, b t l$-Gal4 / $N p^{P 6}$, UAS-matriptase; UAS-HAI-1 (C-C") and $N p^{C 2}$, btl-Gal4 / $N p^{P 6}$, UAS-matriptase; UAS-HAI2 (D-D“) stained with anti-GFP (green) and anti-Spectrin (magenta) antibodies (A-A“), or anti-matriptase (green) and anti-Spectrin (magenta) antibodies (B-D“). While Np::GFP localizes to the intraluminal space (arrow; A-A"), human matriptase only shows intraluminal localization (arrow in D') when co-expressed with human HAI-2 (D-D“). Scale bars correspond to $10 \mu \mathrm{m}$. (E) Percentage of embryos with wild-type-like chitin organization in the mature tracheal aECM (green bars) and tracheal gas-filling (grey bars) are shown for control embryos $\left(N^{C 2}\right.$, btl-Gal4 / +) and $N p$ mutant embryos with btl-Gal4 driven expression of indicated UAS-transgenes. Green bars show data for $\mathrm{n}=40$ embryos for each genotype and grey bars show data for $\mathrm{n}=120$ embryos for each genotype. For method details see chapter 4.3.11. 
matriptase and HAI-2 in $N p$ mutant embryos facilitates intraluminal localization of matriptase (arrow in Fig 28D') and $\sim 90 \%$ of these embryos display wild-type-like organization of tracheal aECM chitin strands and $\sim 57 \%$ show gas filling of the tracheal system (Fig 28E). This rescue requires matriptase, since tracheal expression of human HAI-1 or HAI-2 without matriptase has no effect on the $N p$ mutant phenotype (Fig 28E). These findings indicate that human matriptase substitutes for the functions of $\mathrm{Np}$ in tracheal aECM maturation and gas filling but intraluminal localization of matriptase depends on its co-factor HAI-2. Gas filling of the tracheal system indicates that the tracheal transepithelial barrier is functional and therefore shows that matriptase also substitutes for the role of $N p$ in tracheal epithelial barrier function maintenance. However, it was unclear whether other aspects of the tracheal $N p$ mutant phenotype, i.e. lack of aECM cable contraction and intraluminal Dpy degradation in early-stage 17 (chapter 2.1.8), are also rescued by matriptase. Degradation of intraluminal chitin and Dpy was therefore analyzed in $N p$ mutant embryos with tracheal co-expression of matriptase and HAI-2 by stainings of chitin and Dpy::YFP. Pan-tracheal coexpression of matriptase and HAI-2 mediates formation of a wild-type-like gap between intraluminal chitin cable and tracheal apical plasma membranes in stage $16 \mathrm{~Np}$ mutant embryos (Fig 29A-A"'). The intraluminal chitin cable also contracts in these embryos during formation of the mature tracheal aECM in early-stage 17 (Fig 29B-B'"), as observed in wild-type-embryos (Fig 16B-B'). Finally, the intraluminal chitin and Dpy::YFP cable is removed from the tracheal lumen of $N p$ mutant embryos with tracheal co-expression of matriptase and HAI-2 (Fig 29D-D”), as observed in Np mutant embryos with tracheal expression of Np (Fig 29C-C") and wild-type-like dpy::YFP embryos (Fig 15C-C"). Degradation of Dpy::YFP is specifically mediated by the catalytic activities of $\mathrm{Np}$ and matriptase since tracheal expression of catalytically inactive $\mathrm{Np}^{\mathrm{S} 990 \mathrm{~A}}$ does not mediate degradation of the intraluminal Dpy::YFP cable in $N p$ mutant embryos (Fig 29E-E”).

These rescue experiments indicate that human matriptase substitutes for all functions of $\mathrm{Np}$ during embryonic tracheal development and therefore suggest that $\mathrm{Np}$ and matriptase are functionally homologous. 


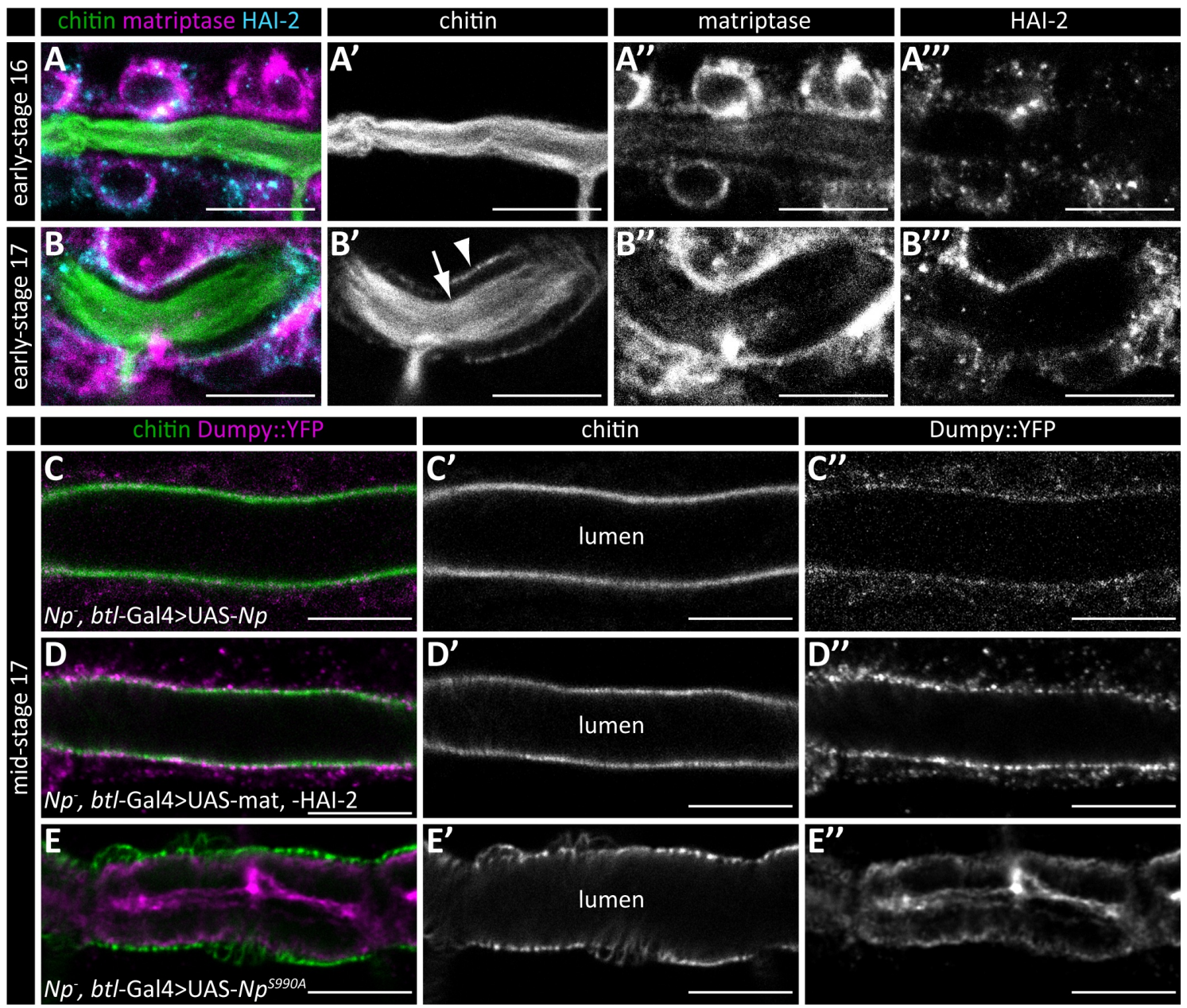

Figure 29: Tracheal intraluminal apical extracellular matrix cable contraction and Dumpy degradation is rescued by human matriptase

(A-B"') Confocal LSM images of tracheal dorsal trunks of $N p^{C 2}$, btl-Gal4 / $N p^{P 6}$, UASmatriptase; UAS-HAI-2 embryos at early-stage 16 (A-A") and early-stage 17 (B-B") stained with FITC-labeled CBP (chitin; green), and anti-matriptase (magenta) and anti-HAI-2 (cyan) antibodies. Formation of a gap between intraluminal chitin cable (arrow in A) and apical plasma membranes (arrowhead in A) is observable. Contraction of the intraluminal chitin cable (arrow in B') during mature aECM (arrowhead in B') maturation in early-stage 17 is visible. Scale bars correspond to $10 \mu \mathrm{m}$. (C-E”) Confocal LSM images of tracheal dorsal trunks of mid-stage $17 N p^{C 2}$, btl-Gal4, dpy::YFP / $N p^{P 6}$, dpy::YFP ; UAS- $N p$ (C-C"), $N p^{C 2}$, $b t l-G a l 4, d p y:$ YFP / $N p^{P 6}$, UAS-matriptase, $d p y:$ YFP ; UAS-HAI-2 (D-D“) and $N p^{C 2}$, btlGal4, dpy::YFP / $N p^{P 6}$, dpy::YFP ; UAS- $N p^{S 990 A}$ (E-E”) embryos stained with FITC-labeld CBP (chitin; green) and anti-GFP antibodies (Dpy::YFP; magenta). Tracheal expression of $N p$ or human matriptase in $N p$ mutant embryos facilitates degradation of the intraluminal Dpy::YFP cable (C-D"). Expression of catalytically inactive $N p^{S 990 A}$ in trachea of $N p$ mutant embryos has no effect on Dpy::YFP cable degradation (E-E”; compare with Fig 15F-G”). Scale bars correspond to $10 \mu \mathrm{m}$. 


\subsubsection{Piopio is proteolytically cleaved by Notopleural and matriptase}

Previous results indicate that proteolytic processing of Pio during embryonic aECM maturation is mediated by $\mathrm{Np}$ (chapter 2.1.12). To test whether $\mathrm{Np}$ and human matriptase share a similar substrate specificity, proteolytic cleavage of Pio by $\mathrm{Np}$ and matriptase was analyzed in cultured $D$. melanogaster cells and in biochemical assays. A pUAST-mCherry::pio plasmid was established for expression of mCherry::Pio fusion protein in cultured D. melanogaster Kc167 cells. The mCherry encoding sequence was inserted into the pio ORF at the equivalent location as in the previously shown genomic knock-in (Fig 18). The cytoplasm of transfected cells was marked by co-expression of GFP for analysis of mCherry::Pio localization.

mCherry::Pio is detectable in cytoplasmic foci and at the plasma membranes (arrowhead in Fig 30A') of transfected D. melanogaster Kc167 cells (Fig 30A-A'). In cells that co-express mCherry::Pio and Np or human matriptase, mCherry::Pio does not localize to the plasma membranes (Fig 30B-B', D-D'). However, low levels of mCherry::Pio are still detectable in the cytoplasm of these cells (Fig 30B-B', D-D'). In contrast, localization of mCherry::Pio to the plasma membrane is not affected by co-expression with catalytically inactive $\mathrm{Np}^{\text {S990A }}$ (arrowhead; Fig 30C-C'). These observations may indicate release of mCherry::Pio from cellular membranes into the cell culture supernatant via proteolytic cleavage by $\mathrm{Np}$ and matriptase, respectively. This is supported by the previous observations that $\mathrm{Np}$ is required for release of Pio into the tracheal lumen (chapter 2.1.10) and mediates proteolytic processing of Pio (chapter 2.1.12) during embryogenesis. Therefore, the effect of $\mathrm{Np}$ and matriptase on proteolytic cleavage of mCherry::Pio and its secretion into the cell culture supernatant was analyzed in cultured D. melanogaster Kc167 cells. Cell lysates and supernatant precipitates of cells that expressed mCherry::Pio or co-expressed mCherry::Pio and $\mathrm{Np}, \mathrm{Np}^{\mathrm{S} 990 \mathrm{~A}}$, or human matriptase were analyzed by SDS-PAGE followed by western blotting and staining with anti-mCherry antibodies.

In lysates of cells that express mCherry::Pio, four mCherry::Pio specific fragments of $\sim 115 \mathrm{kDa}, \sim 90 \mathrm{kDa}, \sim 60 \mathrm{kDa}$ and $\sim 30 \mathrm{kDa}$ are detectable (lane 2 in Fig 30E). While the three largest fragments show identical mass as mCherry::Pio fragments in embryo lysates (Fig 22A), the $\sim 30 \mathrm{kDa}$ fragment presumably corresponds to mCherry. In precipitates of supernatants of cells that express mCherry::Pio, only 


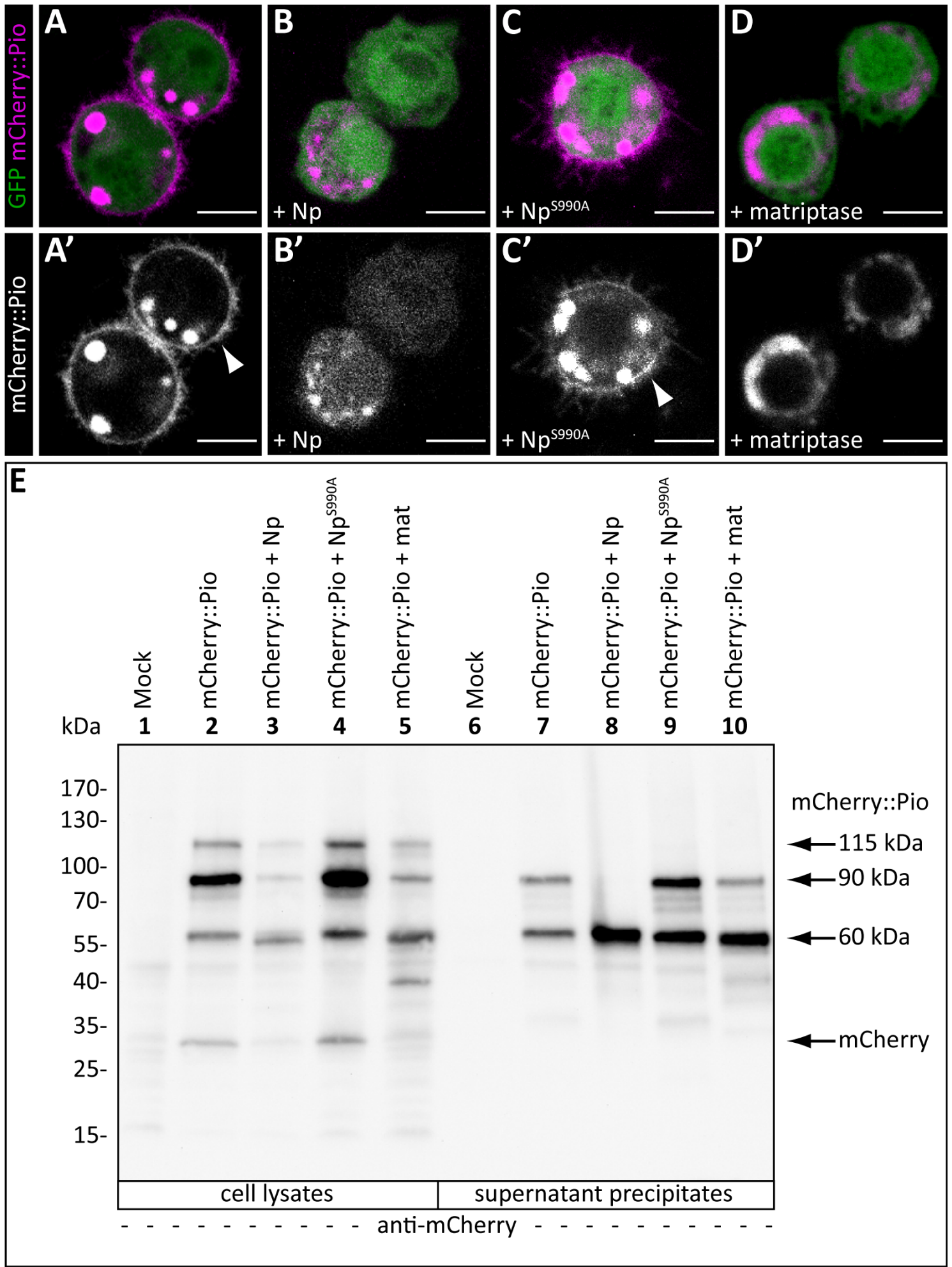

Figure 30: Notopleural and matriptase regulate membrane-anchored localization and proteolytic processing of Piopio in cultured cells

(A-D') Confocal LSM images of cultured D. melanogaster Kc167 cells that express GFP (green) and mCherry::Pio (magenta), and either $\mathrm{Np}$ (B-B'), $\mathrm{Np}^{\mathrm{s990}}$ (C-C'), or human matriptase (D-D'). The UAS/Gal4 system was used for expression. mCherry::Pio localizes to the cytoplasm (marked by GFP) and plasma membranes (arrowheads in $\mathrm{A}^{\prime}, \mathrm{C}^{\prime}$ ) in absence of co-expressed proteases (A-A') or when co-expressed with catalytically inactive $\mathrm{Np}^{\mathrm{S} 990 \mathrm{~A}}$ (C- 
$\left.\mathrm{C}^{\prime}\right)$. Co-expression with $\mathrm{Np}$ (B-B') or human matriptase (D-D') impedes mCherry::Pio localization to the plasma membranes. Scale bars correspond to $5 \mu \mathrm{m}$. (E) Cell lysates (lanes 1-5) and supernatant precipitates (lanes 6-10) of cultured D. melanogaster Kc167 cells were analyzed by SDS-PAGE followed by western blotting and staining with antimCherry antibodies. Cells were either treated with transfection reagent (negative control; Mock; lanes 1 and 6) or transfected with actin5C-Gal4 and pUAST responder plasmids for expression of mCherry::Pio (lanes 2 and 7), mCherry::Pio and $\mathrm{Np}$ (lanes 3 and 8), mCherry::Pio and $\mathrm{Np}^{\mathrm{S} 990 \mathrm{~A}}$ (lanes 4 and 9), or mCherry::Pio and human matriptase (mat; lanes 5 and 10). Co-expression with $\mathrm{Np}$ or human matriptase increases the levels of the $\sim 60$ $\mathrm{kDa}$ mCherry::Pio fragment, in relation to the $\sim 115 \mathrm{kDa}$ and $\sim 90 \mathrm{kDa}$ mCherry::Pio fragments in cell lysates (lanes 3 and 5) and supernatant precipitates (lanes 8 and 10), indicating proteolytic processing of mCherry::Pio.

the two mCherry::Pio specific fragments of $\sim 90 \mathrm{kDa}$ and $\sim 60 \mathrm{kDa}$ are detectable (lane 7 in Fig30E). While the $\sim 90 \mathrm{kDa}$ fragment is most abundant in cell lysates (lane 2), the $\sim 90 \mathrm{kDa}$ and $\sim 60 \mathrm{kDa}$ fragments in supernatant precipitates are present at similar levels (lane 7; Fig 30E). In contrast, only the $\sim 60 \mathrm{kDa}$ mCherry::Pio fragment is detectable in supernatant precipitates of cells that coexpress mCherry::Pio and Np (lane 8 in Fig 30E), and the $\sim 60 \mathrm{kDa}$ fragment shows the highest abundance in lysates of these cells, indicating proteolytic cleavage of mCherry::Pio (lane 3 in Fig 30E). Similarly, co-expression with human matriptase results in increased levels of the $\sim 60 \mathrm{kDa}$ mCherry::Pio fragment in cell lysates and supernatant precipitates and generates a mCherry::Pio fragment at $\sim 40 \mathrm{kDa}$ in cell lysates (lanes 5 and 10 in Fig 30E). The amounts of mCherry::Pio in supernatant precipitates in relation to mCherry::Pio in cell lysates is increased in samples with co-expression of $\mathrm{Np}$ or matriptase (lanes 8 and 10 in Fig 30E), indicating increased release of mCherry::Pio fragments into the supernatant of cultured cells. Catalytically inactive $\mathrm{Np}^{\mathrm{S990A}}$ does not mediate proteolytic processing of mCherry::Pio since the pattern and relative levels of mCherry::Pio fragments are not affected by co-expression with $\mathrm{Np}^{\mathrm{S990A}}$ (lanes 4 and 9 in Fig 30E). These results indicate that $\mathrm{Np}$ and matriptase mediate shedding of Pio from cellular membranes of cultured cells into the cell culture supernatant by proteolytic cleavage. This confirms the previously shown roles of $\mathrm{Np}$ in release of Pio into the tracheal lumen and proteolytic cleavage of Pio in D. melanogaster embryos (chapters 2.1.10 and 2.1.12). However, it was unclear whether $\mathrm{Np}$ and matriptase cleave Pio directly or mediate cleavage of Pio by activation of downstream factors. 
To test whether $\mathrm{Np}$ and matriptase directly cleave Pio, biochemical assays with purified proteases and Pio were performed. Amino-terminally Flag-tagged Pio (Flag::pio; Fig 31E) was expressed in cultured D. melanogaster S2R+ cells and purified via its Flag-tag from cell lysates. Carboxy-terminally Strep-tagged Np (Np::Strep), human matriptase (matriptase::Strep) and their catalytically inactive forms ( $\mathrm{Np}^{\mathrm{S} 990 \mathrm{~A}}::$ Strep and matriptase $\left.{ }^{\mathrm{S} 805 \mathrm{~A}}:: \mathrm{Strep}\right)$ were expressed in cultured $D$. melanogaster S2R+ cells and purified from cell culture supernatants via their Strep-tags. Purified Flag::Pio was then incubated with purified proteases or buffer (negative control) and these assays were subsequently analyzed by SDS-PAGE followed by western blotting and stainings with anti-Flag, anti-Strep and antimatriptase antibodies.

Purified Flag::Pio is mainly detectable as two fragments of $\sim 80 \mathrm{kDa}$ and $\sim 55 \mathrm{kDa}$ and as a faint fragment of $\sim 30 \mathrm{kDa}$ (lane 3 in Fig 31A-B). These three fragments correspond to the three fragments of mCherry::Pio seen in embryo lysates and lysates of $D$. melanogaster Kc167 cells (see Fig 22A and Fig 30E). Incubation of Flag::Pio with purified Np::Strep or matriptase::Strep causes proteolytic cleavage of Flag::Pio, evident by highly increased levels of the $\sim 30 \mathrm{kDa}$ fragment and decreased levels of the $\sim 80 \mathrm{kDa}$ and $\sim 55 \mathrm{kDa}$ fragments (lane 4 in Fig 31A-B). Cleavage depends on the catalytic activity of $\mathrm{Np}$ and matriptase, respectively, since no proteolytic processing of Flag::Pio by catalytically inactive proteases is detectable (lane 5 in Fig 31A-B). To confirm autoactivation of purified Np::Strep and matriptase::Strep, samples of biochemical assays were also analyzed by stainings with anti-Strep (Fig 31C) and anti-matriptase (Fig 31D) antibodies after western blotting. Only a single fragment of $\sim 35 \mathrm{kDa}$ is detectable in samples with purified Np::Strep, corresponding to the predicted mass of the Strep-tagged Np catalytic domain $(32 \mathrm{kDa})$ and therefore indicating activation by cleavage of the zymogen (lanes 1, 4 and 7 in Fig 31C). In contrast, two fragments of $\sim 150 \mathrm{kDa}$ and

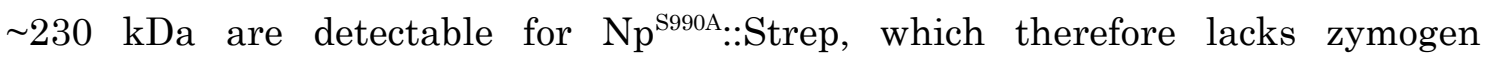
activation (lanes 2, 5 and 8 in Fig 31C). A similar pattern is visible for purified matriptase::Strep and matriptase ${ }^{\mathrm{S} 805 \mathrm{~A}}::$ Strep. A $\sim 32 \mathrm{kDa}$ fragment that corresponds to the predicted mass of the Strep-tagged matriptase catalytic domain ( $31 \mathrm{kDa})$ is detectable in samples with active matriptase::Strep (lanes 1, 4 and 7 in Fig 31D), while catalytically inactive matriptase ${ }^{\mathrm{S} 805 \mathrm{~A}}::$ Strep is detectable only as a $\sim 90 \mathrm{kDa}$ zymogen (lanes 2, 5 and 8 in Fig 31D). These results indicate that Pio is a 


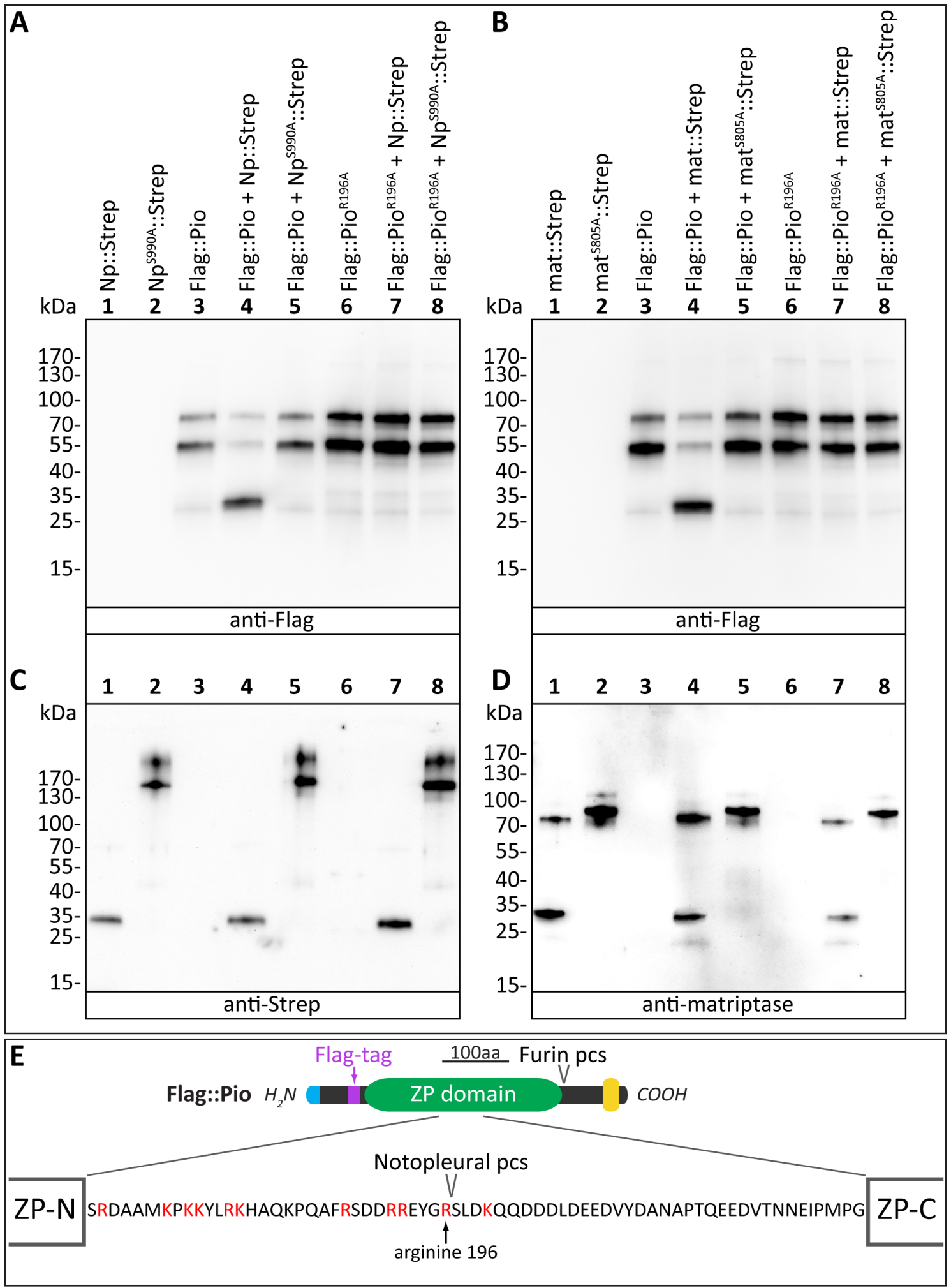

Figure 31: Piopio is cleaved within its zona pellucida domain by Notopleural and matriptase

(A-D) Analysis of biochemical assays by SDS-PAGE followed by western blotting and stainings with anti-Flag (A-B), anti-Strep (C) or anti-matriptase (D) antibodies. The biochemical assays contained purified Flag::Pio or Flag::Pio ${ }^{\mathrm{R} 196 \mathrm{~A}}$ that were incubated with purified Np::Strep or $\mathrm{Np}^{\mathrm{S} 990 \mathrm{~A}}:$ :Strep (A, C), or purified matriptase::Strep or matriptase ${ }^{\mathrm{S} 805 \mathrm{~A}}::$ Strep (B, D). Assays that only contained one of the purified proteins were set up as controls. Proteolytic processing of Flag::Pio is detectable when incubated with 
$\mathrm{Np}::$ Strep (lane 4 in A) or matriptase::Strep (lane 4 in B) by appearance of a $\sim 30 \mathrm{kDa}$ Flag::Pio fragment and decreased abundance of the $\sim 80 \mathrm{kDa}$ and $\sim 55 \mathrm{kDa}$ Flag::Pio fragments. No proteolytic cleavage of Flag::Pio is detectable in assays with catalytically inactive proteases (lanes 5 and 8 in A and B). A single amino acid substitution of arginine 196 with alanine renders Flag::Pio ${ }^{\text {R196A }}$ cleavage resistant to $\mathrm{Np}:$ :Strep (lane 7 in A) and matriptase::Strep (lane 7 in B) since no proteolytic processing is detectable. Stainings with anti-Strep (C) and anti-matriptase (D) antibodies show autocatalytic activation of the proteases. Fragments of the cleaved catalytic domains ( $35 \mathrm{kDa}$ in lanes 1,4 and 7 in $\mathrm{C}$; $\sim 30 \mathrm{kDa}$ in lanes 1,4 and 7 in $\mathrm{D})$ are only detectable for active proteases, while catalytically inactive proteases are detectable as zymogens of $\sim 150-230 \mathrm{kDa}$ and $\sim 90 \mathrm{kDa}$, respectively (lanes 2, 5 and 8 in C-D). (E) Schema of Flag::Pio (top) and amino acid sequence of the linker region between the ZP-N and ZP-C subdomains of the Pio ZPD (bottom). Signal peptide (blue), Flag-tag (purple), ZPD (green) and transmembrane domain (yellow) are indicated. Amino acids that were substituted with alanines by in vitro mutagenesis are shown in red.

substrate for proteolytic cleavage by $\mathrm{Np}$ and human matriptase. Furthermore, the observations confirm autocatalytic mechanisms for activation of $\mathrm{Np}$ (as shown in chapter 2.1.15) and matriptase (Lee et al. 2017). Cleavage of Pio by Np and human matriptase results in a Pio fragment of similar mass, suggesting that both proteases recognize the same protease cleavage site within Pio.

Mass of the Flag::Pio fragment after cleavage by $\mathrm{Np}$ or matriptase suggests that cleavage occurs within the ZPD of Pio. The ZPD is a structurally conserved domain and consists of two subdomains (ZP-N and ZP-C) that are connected by a short linker (Bokhove and Jovine 2018). This linker region is more accessible than other parts of the ZPD (Jovine et al. 2005). While canonical recognition sites for serine proteases consist of eight amino acids and are highly variable, all recognition sites have an arginine or lysine residue in its fourth position (P1 position) and the peptide bond following this residue is hydrolyzed. Furthermore, it is well established that substitution of the $\mathrm{P} 1$ residue with an alanine impedes cleavage by the respective serine proteases (Polgár 2005; Reed et al. 1995). To determine whether the $\mathrm{Np} /$ matriptase protease cleavage site is located in the ZPD linker region of Pio, targeted in vitro mutagenesis of the linker encoding sequence was carried out. Flag::pio constructs that contained mutations to substitute one out of 11 lysines or arginines in the ZPD linker region with alanine were established (Fig 31E). Cleavage of these mutated Flag::Pio proteins by $\mathrm{Np}$ was tested by co- 
expression in cultured $D$. melanogaster cells and subsequent analysis by SDSPAGE, western blotting and stainings with anti-Flag antibodies. Of all tested mutated constructs, only Flag::Pio ${ }^{\text {R196A }}$ (Fig 31E) showed resistance to cleavage by $\mathrm{Np}$ in cultured cells. To confirm these findings, Flag::Pio ${ }^{\text {R196A }}$ was purified and incubated with purified Np::Strep or matriptase::Strep. Indeed, substitution of arginine 196 with alanine renders Flag::Pio ${ }^{\text {R196A }}$ resistant to cleavage by Np::Strep or matriptase::Strep, since $\sim 80 \mathrm{kDa}$ and $\sim 55 \mathrm{kDa}$ fragments of Flag::Pio ${ }^{\mathrm{R} 196 \mathrm{~A}}$ are not affected by incubation with the active proteases (lane 7 in Fig31A-B; compare with lanes 6 and 8). Thus, $\mathrm{Np}$ and human matriptase likely cleave Pio at the same protease cleavage site within the ZPD of Pio and therefore share a similar substrate specificity.

\subsubsection{Proteolytic cleavage of human transforming growth factor $\beta$ receptor type III is mediated by Notopleural and matriptase}

Previous results indicate that Np cleaves the ZPD of Pio (chapters 2.1.12 and 2.1.17) and thereby regulates shedding of the extracellular part of Pio from cellular membranes (chapters 2.1.10 and 2.1.17). In addition, $\mathrm{Np}$ mediates degradation of the ZPD protein Dpy in the tracheal lumen (chapter 2.1.8). Human matriptase can substitute for these functions of $\mathrm{Np}$ (chapters 2.1.16 and 2.1.17), suggesting that proteolytic processing of ZPD proteins by matriptase functional homologs might be an evolutionary conserved mechanism. This is supported by the fact, that ZPD proteins are found in virtually all animals (Jovine et al. 2005). In mammals, ZPD proteins are known to be an essential component of the eponymous Zona Pellucida of oocytes (Gupta 2015). While matriptase is not implicated in oocyte development, many other ZPD proteins are widely expressed in diverse tissues and therefore share expression domains with matriptase. The ZPD protein transforming growth factor $B$ receptor type III (TGFBR3, also known as betaglycan) is expressed almost ubiquitously in human tissues during development and adulthood, and shares many expression domains with matriptase (Szabo et al. 2015; Fagerberg et al. 2014; Wang et al. 1991). While TGFBR3 contains no signaling serine/threonine kinase domain, it acts as a co-receptor for TGF-B receptors type I and II by stabilizing binding of ligands to the receptor complexes (Vander Ark et al. 2018; Bilandzic and Stenvers 2011). Deregulation of TGFBR3 expression or localization 
is implicated in cancer development and progression (Zhang et al. 2016; TodorovicRakovic et al. 2011; Gatza et al. 2010). Interestingly, it is well established that the ectodomain of TGFBR3 can be shedded by proteolytic cleavage and released from cellular membranes into the extracellular space (Elderbroom et al. 2014; Philip et al. 1999). This process plays an important role in down-regulation of TGF-B signaling by extracellular binding of ligands by the TGFBR3 ectodomain. While several proteases were proposed to play a role in TGFBR3 ectodomain shedding, it largely remains elusive which factors mediate proteolytic cleavage of the TGFBR3 ectodomain.

To test whether human matriptase and $D$. melanogaster $\mathrm{Np}$ mediate proteolytic cleavage of human TGFBR3, a pUAST-Strep::TGFBR3::GFP construct was established for co-expression experiments in cultured D. melanogaster cells. Expression of this construct results in a recombinant human TGFBR3 protein with a TwinStrep-tag in its amino-terminal extracellular domain and a carboxyterminally fused GFP at the cytoplasmic end of the protein (Fig 32A). Strep::TGFBR3::GFP was expressed in cultured $D$. melanogaster $\mathrm{S} 2 \mathrm{R}+$ cells either exclusively, or in co-expression with $\mathrm{Np}$ or human matriptase. Cells were fixed and stained with anti-GFP and anti-Strep antibodies to visualize localization of the cytoplasmic domain (GFP) and ectodomain (Strep) of Strep::TGFBR3::GFP. Stainings of the Strep-tagged ectodomain and GFP-labeled cytoplasmic domain of TGFBR3 show complete co-localization and similar signal intensities in cells that express Strep::TGFBR3::GFP (Fig 32B-C"). These cells furthermore display extensive formation of cytoplasmic projections (arrowheads in Fig 32C), which presumably represent filopodia. In cells that co-express $\mathrm{Np}$ and Strep::TGFBR3::GFP, signal intensity of the staining for the Strep-tagged ectodomain is highly reduced, while the GFP-labeled cytoplasmic domain is detectable at similar levels as in cells that only express Strep::TGFBR3::GFP (Fig 32D-E"; compare with Fig 32B-C"). Some areas with GFP localization even show no localization of the Strep-tagged ectodomain at all. Similarly, levels of the Streptagged TGFBR3 ectodomain are highly reduced in cells that co-express matriptase and Strep::TGFBR3::GFP (Fig 32F-G"). Furthermore, cells that co-express Strep::TGFBR3::GFP and Np or matriptase show no formation of cytoplasmic projections and filopodia, respectively (Fig 32D-G"). 


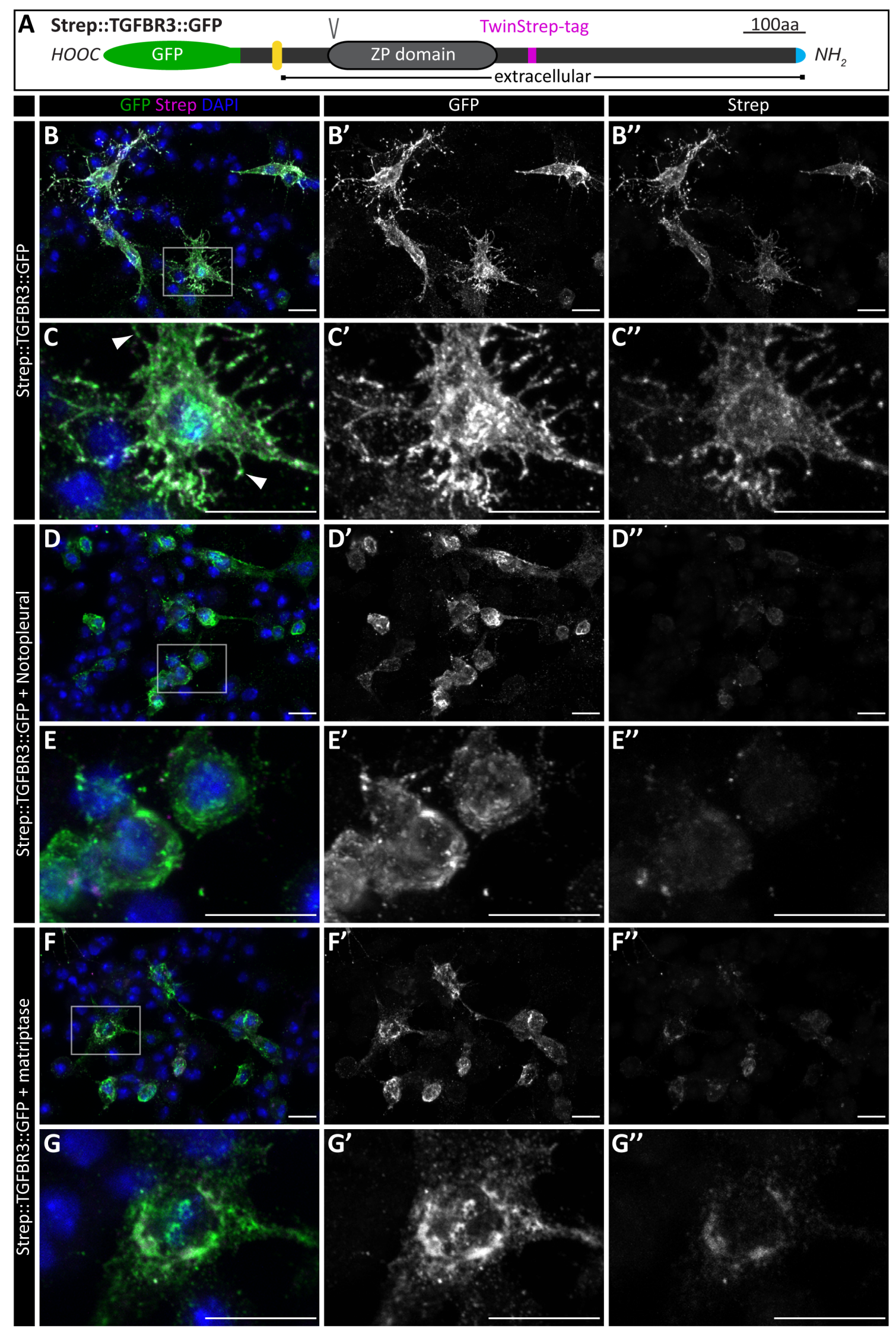

Figure 32: Notopleural and matriptase mediate ectodomain shedding of TGFBR3

(A) Schema of Strep::TGFBR3::GFP fusion protein. GFP (green), transmembrane domain (yellow), Furin pcs (V), ZPD (grey), TwinStrep-tag (magenta) and signal peptide (blue) are 
indicated. (B-G") Confocal LSM z-stack projection images of D. melanogaster S2R+ cells that express Strep::TGFBR3::GFP (B-G"), and either Np (D-E") or human matriptase (FG"), stained with anti-GFP antibodies (green), anti-Strep antibodies (magenta) and DAPI (blue). Areas of details (C-C", E-E", G-G") are indicated by white frames in overview images (B, D, F). All images were acquired with the same settings to enable comparison of signal intensity levels. Cells that express Strep::TGFBR3::GFP display filopodia formation (arrowheads in C). Co-expression of Strep::TGFBR3::GFP with Np or human matriptase leads to highly decreased levels of the Strep-tagged TGFBR3 ectodomain (D-G"). Scale bars correspond to $10 \mu \mathrm{m}$.

The observed decrease in levels of the Strep-tagged TGFBR3 ectodomain in Np or matriptase expressing cells may be due to proteolytic cleavage of TGFBR3. To analyze proteolytic processing of TGFBR3, lysates of cells that expressed Strep::TGFBR3::GFP or co-expressed Strep::TGFBR3::GFP and Np, matriptase, $\mathrm{Np}^{\mathrm{S} 990 \mathrm{~A}}$, or matriptase $\mathrm{S}^{\mathrm{S} 85 \mathrm{~A}}$ were analyzed by SDS-PAGE followed by western blotting and stainings with anti-GFP and anti-Strep antibodies. In lysates of cells that expressed Strep::TGFBR3::GFP, five fragments of $\sim 155 \mathrm{kDa}, \sim 105 \mathrm{kDa}, \sim 50$ $\mathrm{kDa}, \sim 38 \mathrm{kDa}$ and $\sim 28 \mathrm{kDa}$ are detectable by staining of GFP (lane 2 in Fig 33A). The $\sim 155 \mathrm{kDa}, \sim 50 \mathrm{kDa}$ and $\sim 38 \mathrm{kDa}$ fragments show the highest abundance in these lysates and the faint $\sim 28 \mathrm{kDa}$ fragment presumably corresponds to GFP that was not fused to TGFBR3 (lane 2 in Fig 33A). By anti-Strep stainings of these lysates only two fragments of $\sim 155 \mathrm{kDa}$ and $\sim 105 \mathrm{kDa}$ and a faint fragment of $\sim 60$ $\mathrm{kDa}$ are detectable (lane 2 in Fig 33B). Since the $\sim 155 \mathrm{kDa}$ and $\sim 105 \mathrm{kDa}$ fragments are detectable by anti-GFP and anti-Strep stainings, they may correspond to full-length Strep::TGFBR3::GFP of $\sim 155 \mathrm{kDa}$ (predicted mass: 124 $\mathrm{kDa}$ ) and a $\sim 105 \mathrm{kDa}$ Strep::TGFBR3::GFP fragment that was proteolytically processed in the extracellular domain. Discrepancy between predicted and observed mass of full-length Strep::TGFBR3::GFP can be explained by glycosylation of the ectodomain that was shown in several studies (PonceCastañeda et al. 1998; Cheifetz et al. 1988). Co-expression of Strep::TGFBR3::GFP with $\mathrm{Np}$ or matriptase leads to highly decreased levels of the $\sim 155 \mathrm{kDa}$ and $\sim 105$ kDa fragments of Strep::TGFBR3::GFP in cell lysates (lanes 3 and 5 in Fig 33A-B). While levels of all fragments that contain the Strep-tagged ectodomain are highly decreased in these samples (lanes 3 and 5 in Fig 33B), $\sim 50 \mathrm{kDa}$ and $\sim 38 \mathrm{kDa}$ fragments of the GFP-labeled cytoplasmic domain are still detectable (lanes 3 and 5 in Fig 33A), indicating proteolytic cleavage of the ectodomain. Indeed, co- 


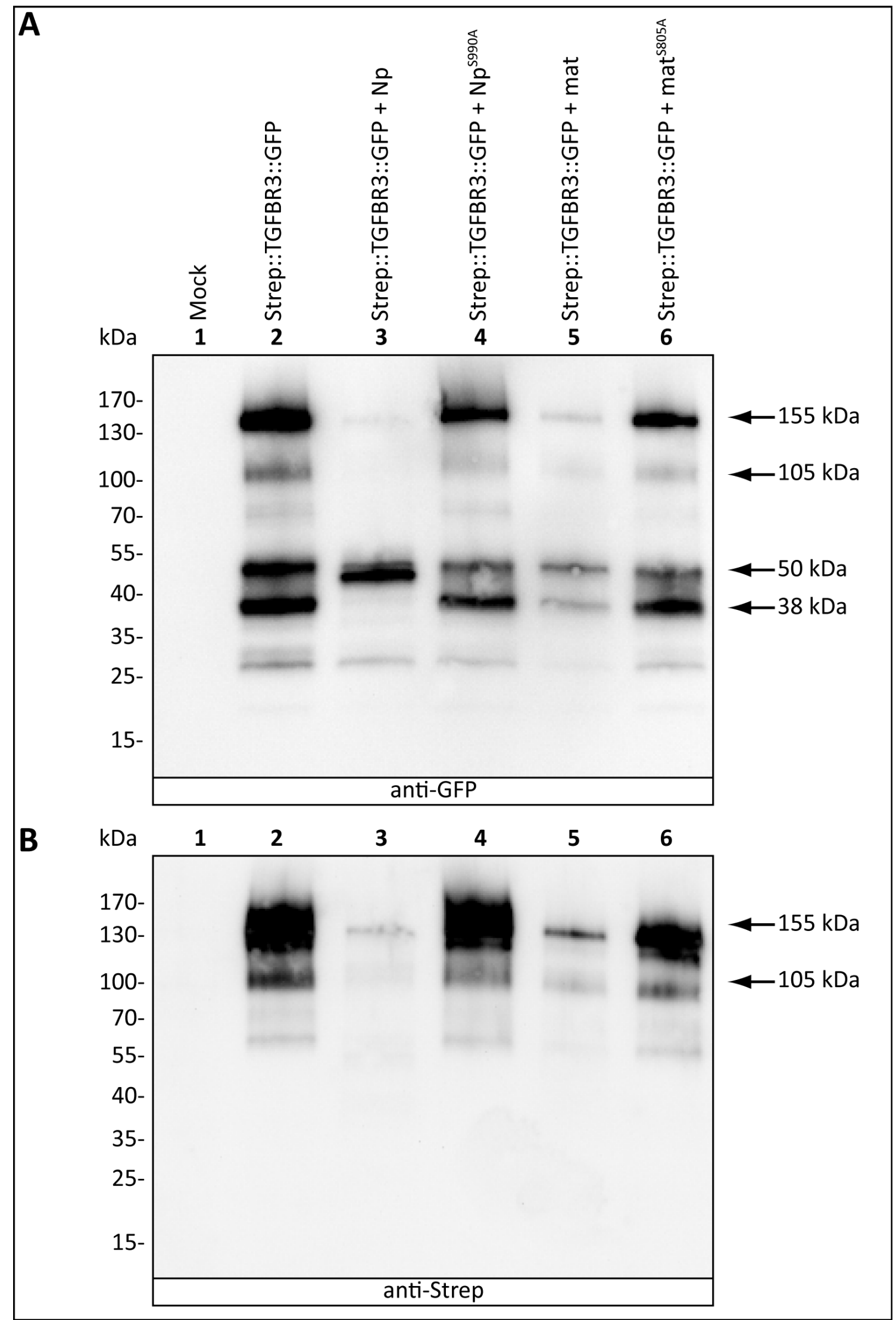

Figure 33: Proteolytic processing of TGFBR3 is mediated by Notopleural and matriptase

Cell lysates of cultured D. melanogaster S2R+ cells were analyzed by SDS-PAGE and subsequent western blotting and stainings with anti-GFP (A) or anti-Strep (B) antibodies. 
Cells were either treated with transfection reagent (negative control; Mock; lane 1) or transfected with actin 5C-Gal4 a n d $p U A S T$ responder plasmids for expression of Strep::TGFBR3::GFP (lane 2), Strep::TGFBR3::GFP and Np (lane 3), Strep::TGFBR3::GFP and $\mathrm{Np}^{\text {S990A }}$ (lane 4), Strep::TGFBR3::GFP and matriptase (lane 5) or Strep::TGFBR3::GFP and matriptase $\mathrm{e}^{\mathrm{S} 805 \mathrm{~A}}$ (lane 6). Co-expression of Strep::TGFBR3::GFP with Np or matriptase leads to decreased levels of the $\sim 155 \mathrm{kDa}$ and $\sim 105 \mathrm{kDa}$ fragments of Strep::TGFBR3::GFP (lanes 3 and 5) in stainings with anti-GFP (A) and anti-Strep (B) antibodies, indicating loss of the Strep-tagged TGFBR3 ectodomain. Loss of the Strep-tagged TGFBR3 ectodomain is not observable in lysates of cells that expressed catalytically inactive proteases (lanes 4 and 6) and therefore depends on the catalytic activity of $\mathrm{Np}$ and matriptase, respectively.

expression of Strep::TGFBR3::GFP with catalytically inactive $\mathrm{Np}^{\text {S990A }}$ or matriptase $^{\mathrm{S} 805 \mathrm{~A}}$ does not affect the pattern of Strep::TGFBR3::GFP fragments or levels of the Strep-tagged TGFBR3 ectodomain (lanes 4 and 6 in Fig 33A-B; compare with lane 2 in Fig 33A-B).

These experiments indicate that $\mathrm{Np}$ and matriptase mediate proteolytic processing of human TGFBR3 that results in loss of the TGFBR3 ectodomain from cells, likely representing ectodomain shedding of TGFBR3. Furthermore, the observations that expression of TGFBR3 induces formation of filopodia which is suppressed by coexpression with $\mathrm{Np}$ or matriptase suggests a role of TGFBR3 ectodomain shedding in filopodia formation. 


\subsection{Functional analysis of CG4386 during embryogenesis}

A detailed functional analysis of CG4386 during embryogenesis was carried out with similar methods as previously used for functional analysis of $N p$ in chapter 2.1. CRISPR/Cas9 technology was used to generate loss-of-function alleles of $C G 4386$ and a targeted insertion of a fluorophore encoding sequence into the ORF of $C G 4386$ to visualize protein localization. $C G 4386$ mutant embryos were analyzed by in vivo observations, immunohistochemical stainings and transmission electron microscopy. With these methods, morphology of the tracheal epithelium and aECM in CG4386 mutant embryos were examined. Furthermore, the UAS/Gal4 system was used for tissue-specific rescue experiments to determine whether human prostasin is functionally homologous to the serine protease encoded by CG4386. Finally, in vitro experiments with purified proteins were performed to elucidate interactions of the CG4386 encoded serine protease with Np. In a first step, an analysis of $C G 4386$ expression during embryogenesis and an in silico analysis of the serine protease encoded by $C G 4386$ were carried out.

\subsubsection{CG4386 encodes a trachea specific serine protease with sequence similarities to vertebrate prostasin}

As shown in chapter 2.1, pan-tracheal RNAi-mediated knock-down of CG4386 leads to lack of tracheal gas filling, indicating a role of $C G 4386$ in tracheal development. M. Jaspers analyzed the expression pattern of $C G 4386$ during embryogenesis by whole-mount RNA in situ hybridization stainings of wild-type embryos with a digoxigenin-labeled CG4386 antisense probe (Jaspers 2011). Strikingly, CG4386 transcripts are exclusively detectable in cells of the tracheal system (Fig 34). Expression of $C G 4386$ is first detectable in posterior segments of the tracheal system of stage 15 embryos (Fig 34A-B). Expression then spreads to the whole tracheal system during stage 16 (Fig 34C) and increases until stage 17 of embryogenesis (Fig 34D). Thus, expression of CG4386 is restricted to the tracheal system during embryogenesis.

To determine the protein domain organization of the serine protease encoded by CG4386 and identify putative orthologs in other species, an in silico analysis was performed. $C G 4386$ encodes a single putative 372 amino acids protein. In silico 


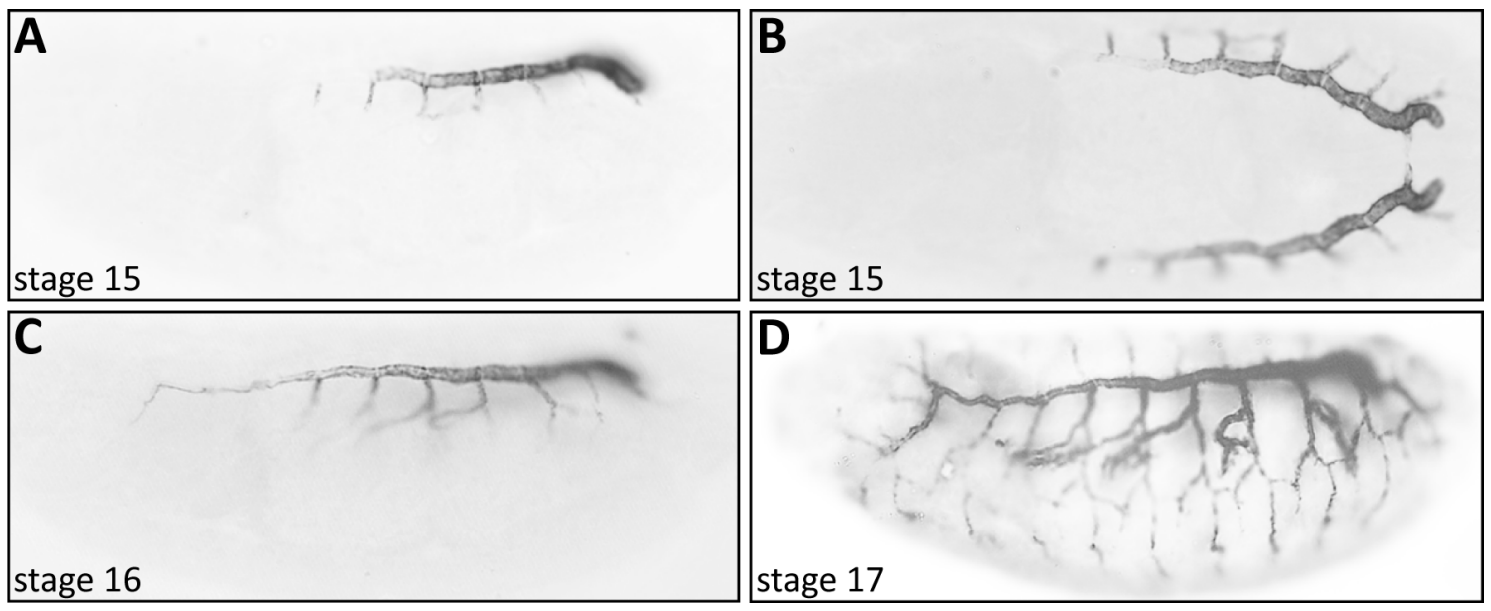

Figure 34: CG4386 is exclusively expressed in the tracheal system during embryogenesis

Bright field light microscopic images of whole-mount in situ RNA-hybridization stainings of wild-type embryos with a digoxigenin-labeled $C G 4386$ antisense probe. $C G 4386$ transcripts are detectable in cells of posterior segments of the tracheal system during stage 15 (lateral view in A, dorsal view in B). CG4386 expression is detectable in the whole tracheal system of stage 16 and stage 17 embryos and expression levels increase until stage 17 (C-D). Images were adapted from Jaspers (2011).

analysis predicts an amino-terminal signal peptide and a carboxy-terminal trypsinlike serine protease catalytic domain (Fig 35A). The serine protease domain contains a conserved cleavage site for zymogen activation at its amino-terminal end ( $\mathrm{V}$ in Fig 35A) and conserved cysteines that putatively form a disulphide bridge between the catalytic domain and amino-terminal pro-domain (Fig 35A). Previous results indicate that $D$. melanogaster $\mathrm{Np}$ is functionally homologous to human matriptase (chapters 2.1 .16 - 2.1.18). One of the most essential interaction partners of vertebrate matriptase is prostasin, a GPI-anchored serine protease that is activated by matriptase and reciprocally contributes to matriptase activation and ectodomain shedding (Friis et al. 2014; Miller and List 2013). Interestingly, in a comparison of human prostasin to D. melanogaster proteins using BLASTP (Altschul et al. 1997), human prostasin shows the highest degree of sequence similarity to the serine protease encoded by $C G 4386$. Therefore, $C G 4386$ will be referred to as tracheal-prostasin (tpr) below, due to its tracheal system-specific expression and sequence similarity of the encoded protease to human prostasin. While Tpr contains no predicted GPI anchor site, it shares a $35 \%$ sequence identity with human prostasin (Fig 35). The PRSS8 gene that encodes prostasin is highly conserved in vertebrates (Szabo and Bugge 2011). Accordingly, prostasin homologs from $M$. mullatus, $M$. musculus, $R$. norvegicus and $B$. taurus show the highest 
sequence similarities to $D$. melanogaster $\mathrm{Tpr}$ in BLASTP queries. These observations imply that tpr encodes a serine protease that is related to vertebrate prostasin.

\begin{tabular}{|c|c|}
\hline Drosophila Trach & $\begin{array}{l}\text { leal-prostasin (Tpr) } \underline{50 \text { aa }} \\
\text { catalytic domain } \mathrm{COOH} \quad \mathrm{H}_{2} \mathrm{~N} \text { sequence identity }{ }^{s-s} \mathrm{I}\end{array}$ \\
\hline $\begin{array}{l}\text { B } \\
\text { Tracheal-prostasin } \\
\text { prostasin }\end{array}$ & MNRAWLCLLICLALSCGPSQSASQDRATNQTAASPLLKQSQNTF IQWVLSLLPQRPGSSDSEN \\
\hline $\begin{array}{l}\text { Tracheal-prostasin } \\
\text { prostasin }\end{array}$ & 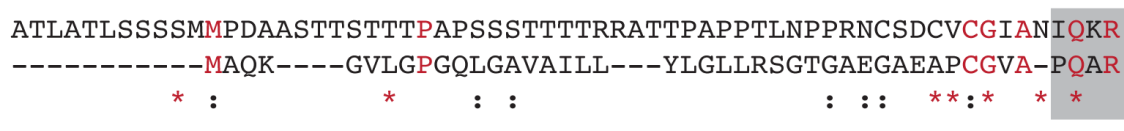 \\
\hline $\begin{array}{l}\text { Tracheal-prostasin } \\
\text { prostasin }\end{array}$ & 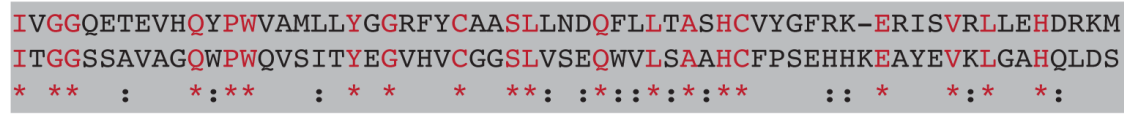 \\
\hline $\begin{array}{l}\text { Tracheal-prostasin } \\
\text { prostasin }\end{array}$ & 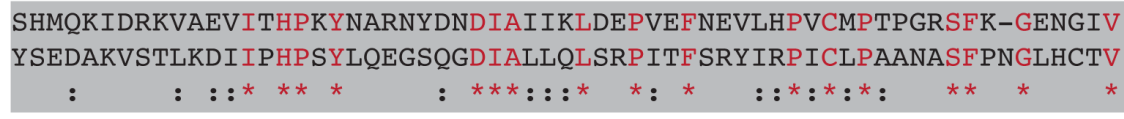 \\
\hline $\begin{array}{l}\text { Tracheal-prostasin } \\
\text { prostasin }\end{array}$ & 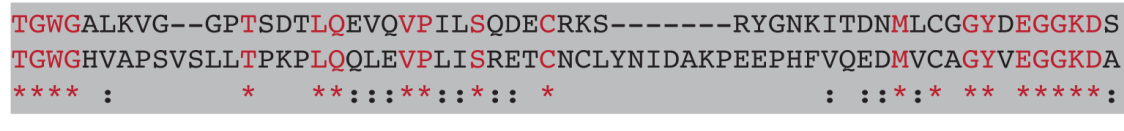 \\
\hline $\begin{array}{l}\text { Tracheal-prostasin } \\
\text { prostasin }\end{array}$ & 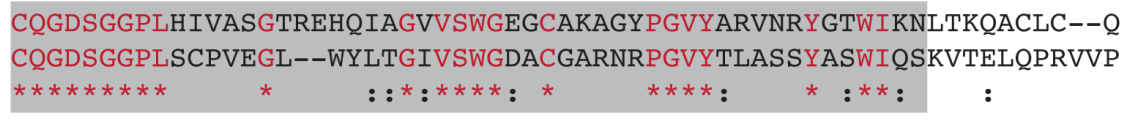 \\
\hline $\begin{array}{l}\text { Tracheal-prostasin } \\
\text { prostasin }\end{array}$ & $\begin{array}{l}\text { Q--ETKKIK-1K } \\
\text { QTQESQPDSNLCGSHAFSSAPAQGLLRPILFLPLGLALGLLSPWLSEH } \\
* \quad *::\end{array}$ \\
\hline
\end{tabular}

Figure 35: CG4386 encodes a serine protease with sequence similarities to human prostasin and is therefore named tracheal-prostasin (tpr)

(A) Schemata of the protein domain organizations of D. melanogaster Tracheal-prostasin (Tpr) and human prostasin. The signal peptides (blue), catalytic serine protease domains (green), GPI-anchor site (red), conserved disulphide bridges (-S-S-) and the zymogen activation cleavage sites $(\mathrm{V})$ are indicated. (B) Sequence alignment of D. melanogaster Tpr and human prostasin by Clustal omega (Sievers et al. 2011) reveals a $35 \%$ sequence identity (red; asterisks) and additional $19 \%$ sequence similarity (colon). Catalytic domains are highlighted in grey.

\subsubsection{Tracheal-prostasin is essential for gas filling of the tracheal system}

To analyze the role of tpr during embryogenesis, loss-of-function alleles for tpr were generated using the CRISPR/Cas9 system (Fig 36A-C). The obtained $t_{p r}{ }^{D 1}$ and $t p r^{F 1}$ alleles cause early frame-shifts in the ORF followed by premature 
translation stops (Fig 36B-C). Furthermore, the $t p r^{D 1}$ and $t p r^{F 1}$ alleles fail to complement each other as well as the Df(2R)BSC664 chromosome in which the region 57D12-58A3 is deleted, including tpr. Embryos that are homozygous for the $t p r^{D 1}$ or $t p r^{F 1}$ alleles or transheterozygous for $t p r^{D 1} / t p r^{F 1}$ mostly lack tracheal gas filling (Fig 36D). These mutant embryos show otherwise wild-type-like embryonic development and hatch as larvae that die during first instar larval stage. Approximately $90 \%$ of the tpr mutant larvae show no LC of the tracheal system
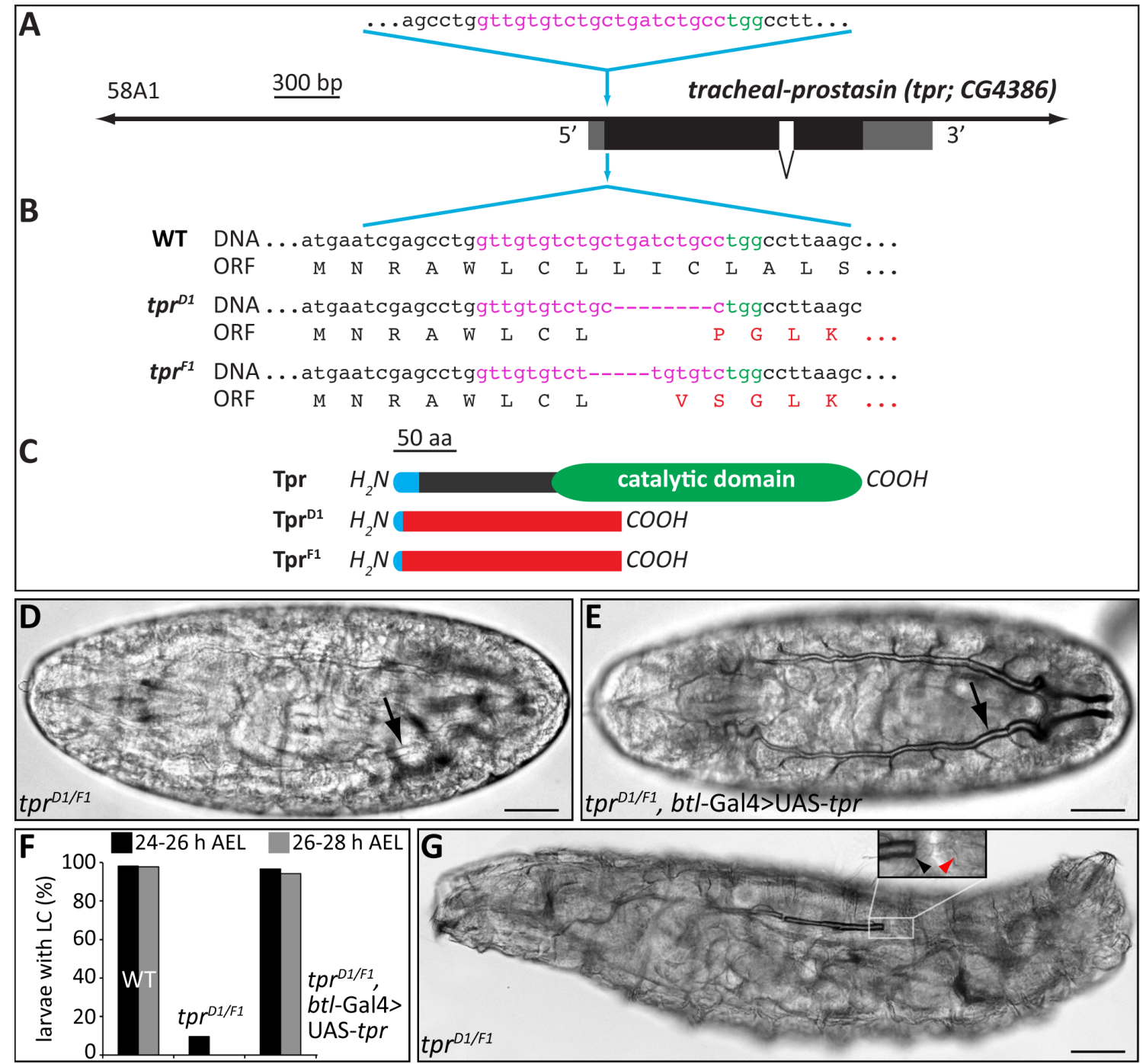

Figure 36: Generation of tracheal-prostasin loss-of-function alleles by CRISPR/Cas9-mediated mutagenesis

(A-C) Schema of CRISPR/Cas9-mediated mutagenesis to generate frame shift mutations in the 5' region of the tpr ORF. (A) Physical map of tpr genomic region shows the sgRNA recognition site (magenta letters) with PAM (green letters), translated DNA (black boxes) and UTRs (grey boxes). (B) Generated DNA indels cause ORF frame shifts in $t p r^{D 1}$ and $t p r^{F 1}$ mutant alleles. (C) Frame shifts result in expression of truncated Tpr proteins. Signal peptides (blue), catalytic serine protease domain (green) and regions with frame-shifted amino acid sequences (red) are indicated. (D-E) Bright field light microscopic images of 
stage $17 t^{D r} r^{D 1} / t^{F 1} r^{F 1}$ (D) and $t p r^{D 1}, b t l-G a l 4 / t p r^{F 1}$;UAS-tpr (E) mutant embryos. While tpr mutant embryos show no tracheal LC (arrow in D), tpr mutant embryos with tracheal expression of tpr display gas filling of the tracheal system (arrow in E). Scale bars correspond to $50 \mu \mathrm{m}$. (F) Quantification of wild-type (WT), $t p r^{D 1} / t p r^{F 1}$ mutant and $t p r^{D 1}$, btlGal4/tpr ${ }^{F 1}$;UAS- $t$ p $r$ mutant larvae with LC. For each genotype, $\mathrm{n}=150$. Larvae were analyzed immediately after hatching at $24-26$ hours after-egg-laying (AEL) and at $26-28$ hours AEL. Approximately $10 \%$ of tpr mutant larvae show a gas-filled tracheal system at $24-26$ hours AEL but no tpr mutant larvae with gas-filled trachea are present at $26-28$ hours AEL. (H) Bright field light microscopic image of a tpr mutant larvae with partially gas-filled trachea. Detail shows that parts of the trachea are still gas-filled (black arrowhead) while adjacent parts are refilled with liquid (red arrowhead). Scale bar corresponds to $50 \mu \mathrm{m}$.

when hatching (Fig 36F). The approximately $10 \%$ of tpr mutant larvae that hatch with a gas-filled tracheal system display refilling of the trachea with liquid within 4 hours after hatching and also die during the first instar larval stage (Fig 36F-G). To test whether the observed lack of tracheal gas filling was indeed caused by the CRISPR/Cas9 mediated mutations in tpr, tracheal system specific rescue experiments were performed. UAS responder lines for tpr were established and used for btl-Gal4 driven pan-tracheal expression in tpr mutant embryos. Ectopic tracheal tpr expression from the UAS transgene in tpr mutant embryos results in wild-type-like gas filling of the tracheal system (Fig 36E-F). Furthermore, these embryos develop to wild-type-like, fertile flies. The rescue experiments show that the observed tracheal LC phenotype in tpr mutant embryos and lethality of tpr mutant larvae are due to loss of tpr expression and were not caused by off-target effects during CRISPR/Cas9-mediated mutagenesis. In conclusion, tpr is essential for tracheal gas filling and has no essential role apart from the tracheal system.

\subsubsection{Tracheal-prostasin localizes to the tracheal apical extracellular matrix}

To visualize Tpr protein expression and localization, a tpr::mCherry knock-in into the endogenous tpr locus was generated (Fig 37). First, the tpr genomic region was exchanged with an attP site and a 3xP3-DsRed selection marker gene using CRISPR/Cas9 mediated homology-directed repair (Fig 37A-B). The 3xP3-DsRed selection marker was removed by Cre recombinase-mediated excision, generating the $t p r^{a t t P}$ allele (Fig 37B). Homozygous $t p r^{a t t P}$ larvae display lack of tracheal gas 


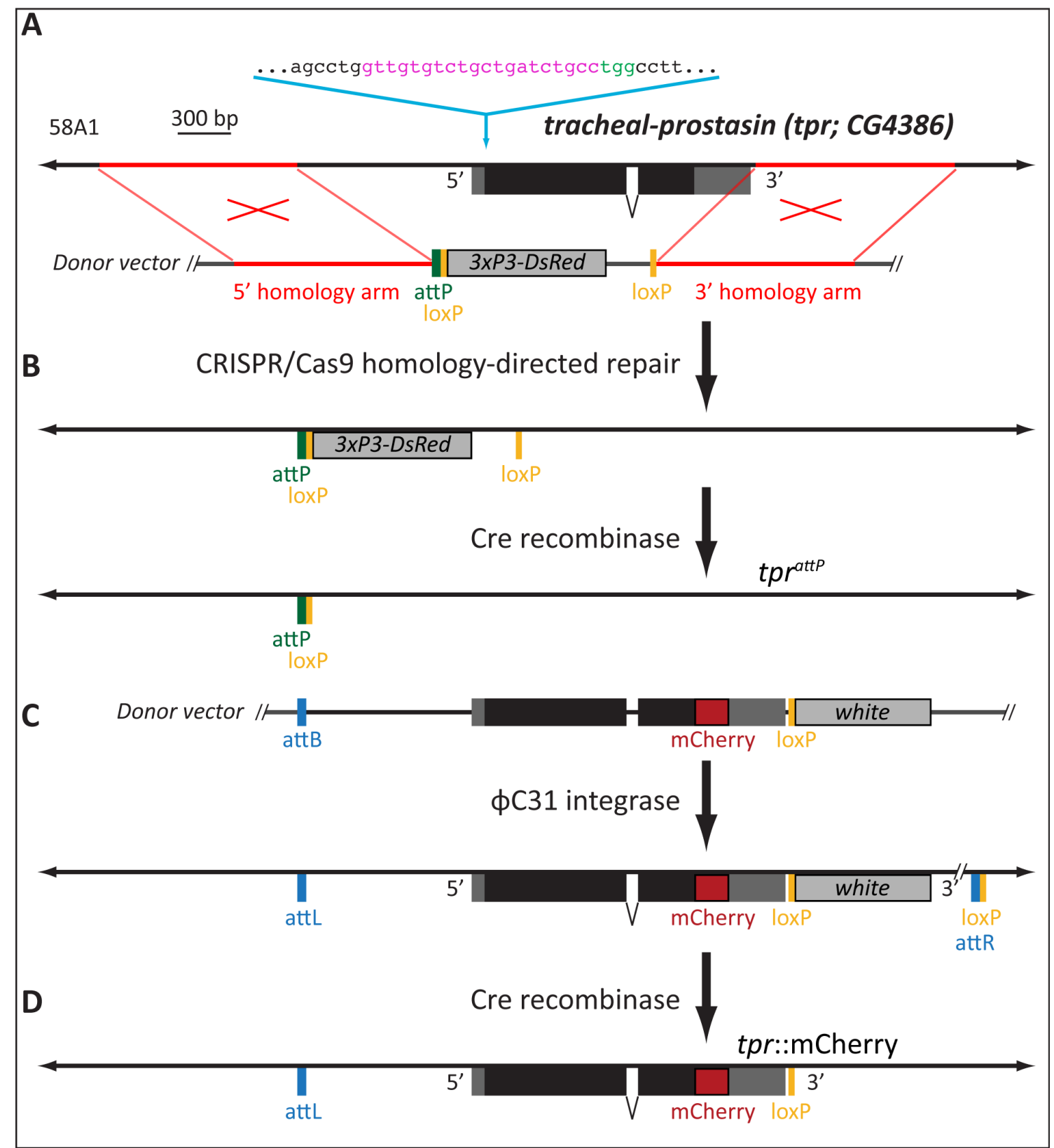

Figure 37: Generation of flies with endogenous expression of Tracheal-prostasin::mCherry using CRISPR/Cas9 technology.

(A) Schema of the tpr genomic locus together with a donor vector containing two homology arms (red), a 3xP3-DsRed selection marker gene, an attP site (green), and two lox $P$ sites (yellow). The sgRNA recognition site (magenta letters), PAM (green letters), translated DNA (black boxes) and UTRs (grey boxes) are indicated. (B) tpr locus after CRISPR/Cas9mediated homology-directed repair (top) and Cre recombinase-mediated 3xP3-DsRed gene excision (bottom), producing the $t p r^{\text {att } P}$ allele. (C) Donor vector (top) for $\Phi C 31$-integrasemediated knock-in of tpr genomic region with a mCherry encoding sequence integrated 3 ' to the tpr ORF, generating white ${ }^{+}$flies that express mCherry-labeled Tpr (bottom). (D) tpr::mCherry allele after Cre recombinase-mediated white gene excision. 
filling after hatching and die during first instar larval stage, as previously reported for other tpr loss-of-function alleles (chapter 2.2.2). The ФC31 integrase attP/attB system was used to knock-in the tpr genomic region that was deleted in the previous step together with a mCherry encoding sequence inserted after the last protein encoding tpr exon and a white selection marker gene (Fig 37C). Cre recombinase-mediated white gene excision then generated the tpr::mCherry allele (Fig 37D). Embryos that are homozygous for tpr::mCherry show wild-type-like gas filling of the tracheal system and develop to fertile adult flies, indicating normal function of the encoded Tpr::mCherry fusion protein.

To visualize Tpr::mCherry localization, homozygous tpr::mCherry embryos were stained with anti-mCherry and anti-Spectrin antibodies. Tpr::mCherry is exclusively detectable in the tracheal system during embryogenesis and is present in all branches of the tracheal network of stage 16 and 17 embryos (Fig 38A-B). It localizes to the tracheal intraluminal aECM (arrows in Fig 38C', D') and apical plasma membranes (arrowheads in Fig 38C', D') during stages 16 and 17, while
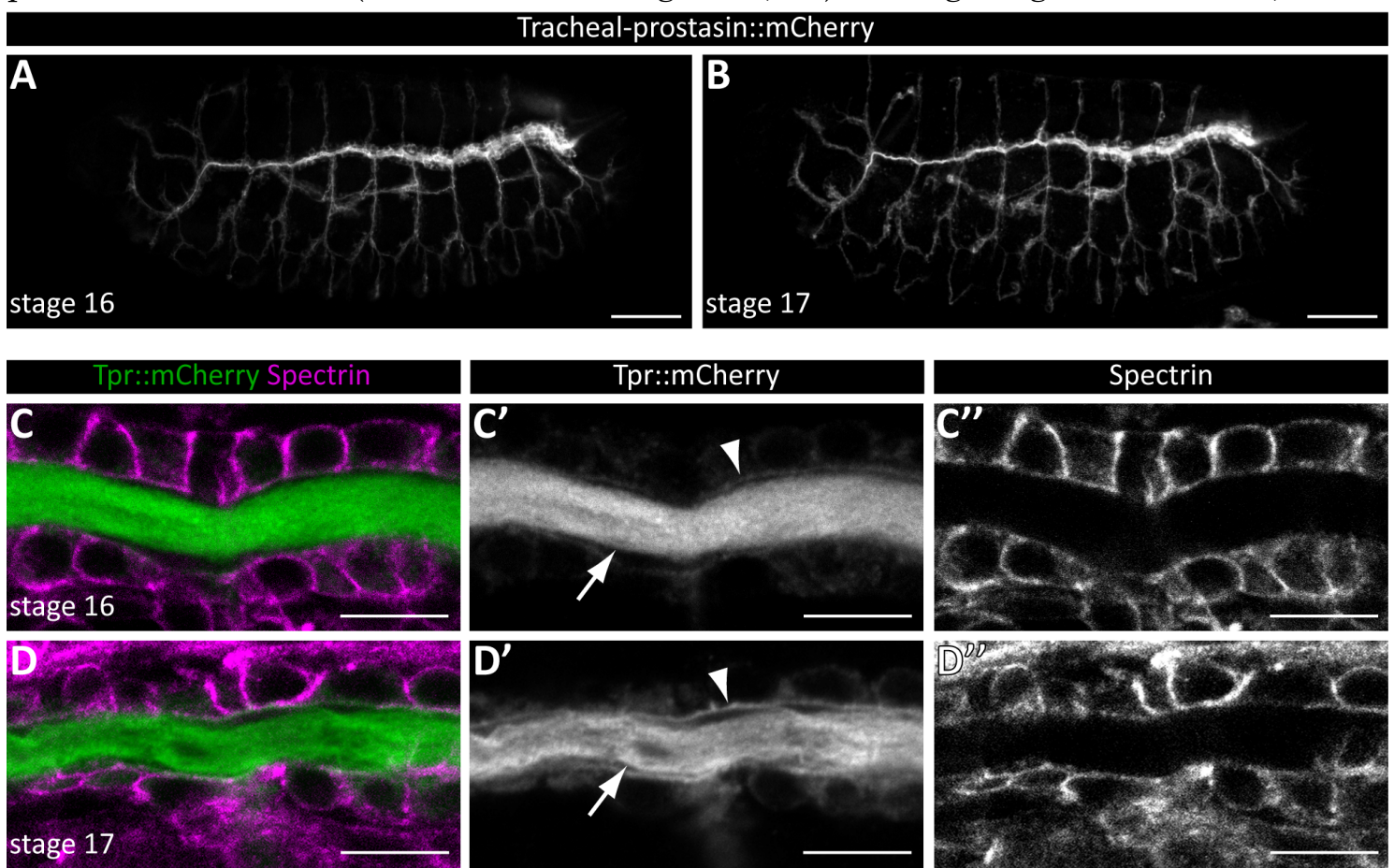

Figure 38: Tracheal-prostasin localizes to apical plasma membranes and the apical extracellular matrix in the tracheal system

Confocal LSM images of stage 16 (A, C-C") and stage 17 (B, D-D") tpr::mCherry embryos (A-B) and their tracheal dorsal trunks (C-D"), that were stained with anti-mCherry (green) and anti-Spectrin (magenta) antibodies. Tpr::mCherry is exclusively detectable in the tracheal system during embryogenesis (A-B) and localizes to the intraluminal aECM (arrows) and apical plasma membranes (arrowheads; C-D"). Scale bars correspond to $50 \mu \mathrm{m}$ (A-B) and $10 \mu \mathrm{m}(\mathrm{C}-\mathrm{D} ")$, respectively. 
membrane-associated levels are increased in trachea of stage 17 embryos (Fig 38CD"). These observations demonstrate that Tpr is a component of the tracheal intraluminal aECM and shows a similar tracheal localization as $\mathrm{Np}$ during stages 16 and 17 (see Fig 8E-F"). Furthermore, Tpr::mCherry localization confirms that tpr expression is restricted to the tracheal system during embryonic development, as shown by RNA in situ hybridization stainings (Fig 34).

\subsubsection{Tracheal network formation, transepithelial barrier function and epithelial polarization are not dependent on tracheal-prostasin}

To test whether lack of tracheal gas filling in tpr mutant embryos (chapter 2.2.2) is caused by tracheal network formation defects or a lack of transepithelial barrier function, stage 16 tpr mutant embryos were stained for chitin to visualize the tracheal network, and were injected with Texas Red-labeld $10 \mathrm{kDa}$ dextran to test tracheal epithelial barrier function. Staining of the intraluminal chitin cable of stage 16 tpr mutant embryos reveals wild-type-like tracheal network formation and continuous integrity of the tracheal system (Fig 39A; compare with wild-type in Fig 9A). Furthermore, Texas Red-labeled $10 \mathrm{kDa}$ dextran that was injected into the haemocoel of stage 16 tpr mutant embryos does not diffuse into the tracheal lumen (arrow in Fig 39B) as it was previously observed in wild-type embryos (Fig $9 \mathrm{C})$. These results indicate that tracheal network formation and tracheal transepithelial barrier function are not dependent on tpr.
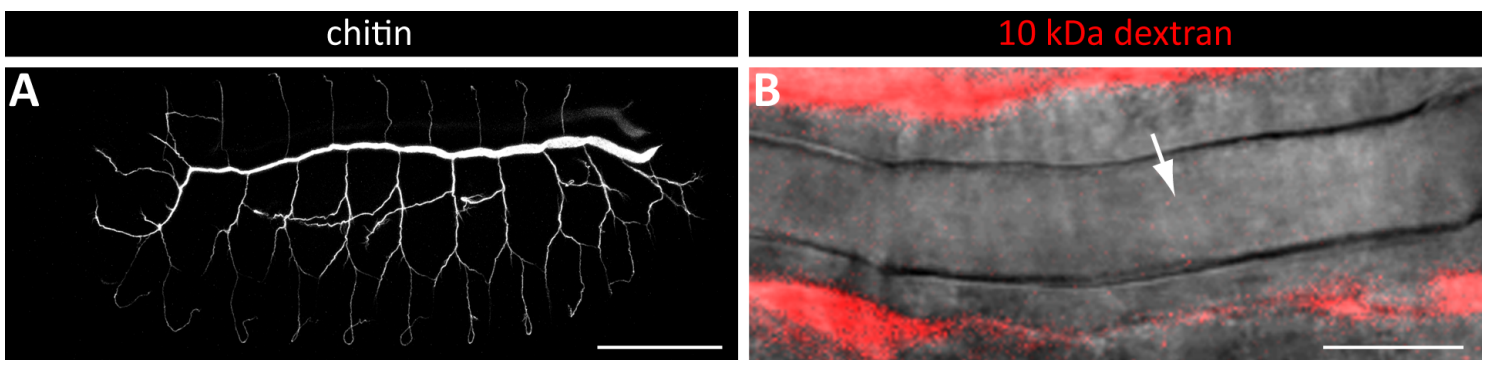

Figure 39: Tracheal-prostasin is not required for tracheal network formation and transepithelial barrier function

(A) Confocal LSM z-stack projection image of a stage $16 \mathrm{tpr}^{D 1} / \mathrm{tpr}^{F 1}$ mutant embryos that was stained with CBP (chitin). The tracheal network of tpr mutant embryos is wild-typelike (compare to Fig 9A). Scale bar corresponds to $50 \mu \mathrm{m}$. (B) In vivo LSM confocal image of a tracheal dorsal trunk of a stage $16 \mathrm{tpr}^{D 1} / \mathrm{tpr}^{F 1}$ mutant embryo after Texas Red-labeled 10 $\mathrm{kDa}$ dextran injection into the haemocoel. Dextran (red) does not diffuse into the dorsal trunk lumen (arrow). Scale bar corresponds to $10 \mu \mathrm{m}$. 
Due to these observations, defective network formation and lack of transepithelial barrier function can be ruled out as reasons for lack of tracheal gas filling in tpr mutant embryos (chapter 2.2.2). Other reasons for the observed lack of tracheal LC in tpr mutant embryos may be a failure of tracheal mature aECM formation or defects in epithelial development. To visualize tracheal aECM organization, epithelial polarization and localization of the septate junctions in tpr mutant embryos, stainings for chitin, the apical membrane marker Uif and septate

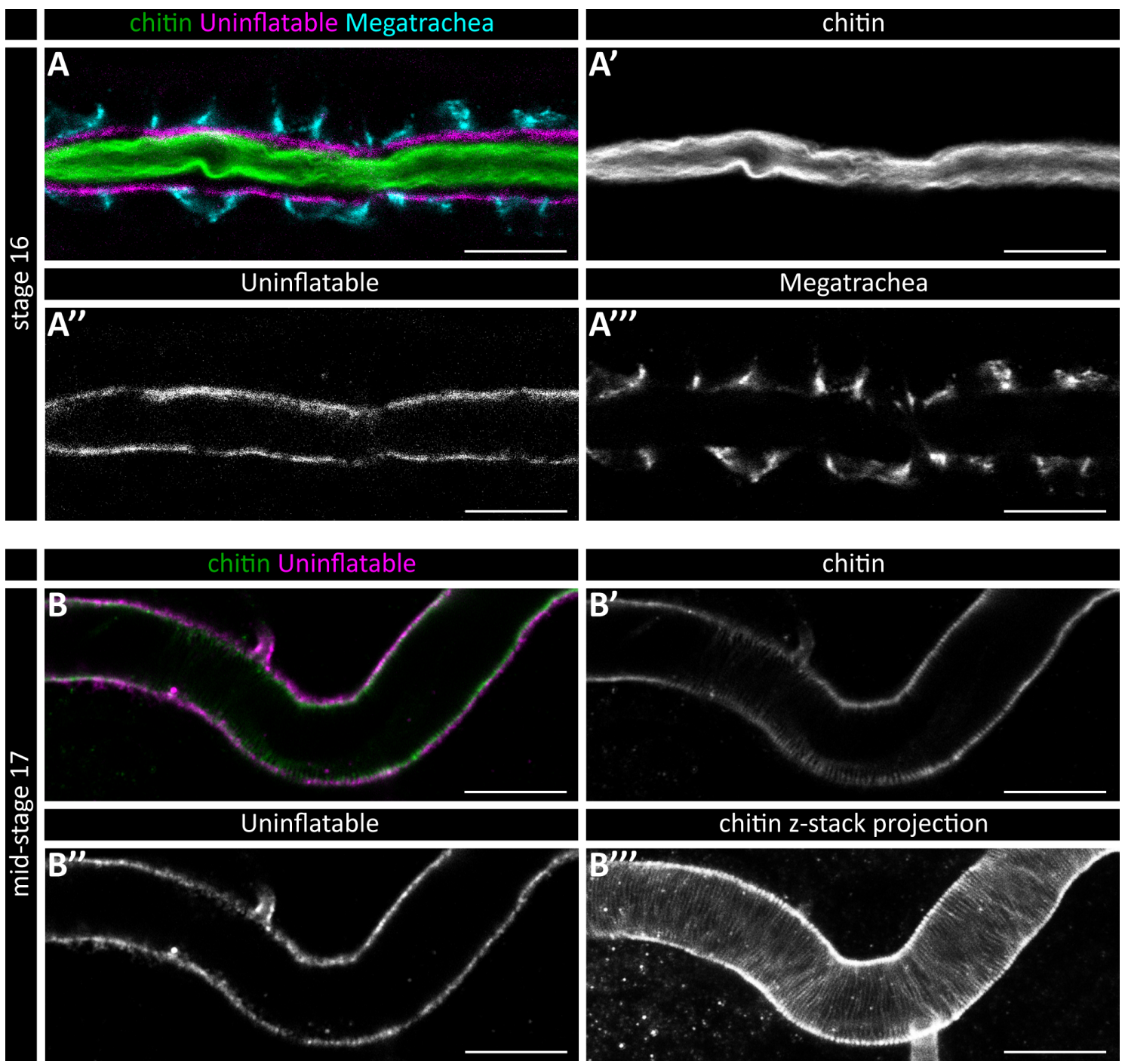

Figure 40: Tracheal-prostasin is not required for tracheal epithelial polarization, septate junction formation and chitin organization

Confocal LSM images (A-B") and z-stack projection image (B"') of tracheal dorsal trunks of stage 16 (A-A"') and mid-stage 17 (B-B"') $t p r^{D 1} / t p r^{F 1}$ mutant embryos that were stained with CBP (chitin; green), anti-Uif (magenta) and anit-Mega (cyan) antibodies. Mega staining is only shown for stage 16 (A, A"'). All stained components show wild-type-like localization (compare with wild-type in Fig 10E-E" and Fig 16C-C"). Notably, chitin strands of the mature tracheal aECM also display a wild-type-like organization in tpr mutant embryos (B"'; compare with wild-type in Fig 13C). Scale bars correspond to $10 \mu \mathrm{m}$. 
junction marker Mega were performed. Uif and Mega show wild-type-like localization in the tracheal system of stage 16 tpr mutant embryos and the intraluminal chitin cable forms normally and shows wild-type-like spatial separation from the apical plasma membranes (Fig 40A-A"'; compare with wildtype in Fig 10E-E"). In mid-stage 17 tpr mutant embryos, degradation of the intraluminal chitin cable is completed and chitin strands of the mature tracheal aECM are present at the apical surface of tracheal cells (Fig 40B-B"). The chitin strands of of tpr mutant trachea display a wild-type-like regularly-spaced parallel organization (Fig 40B"'; compare with wild-type in Fig 13A, C). These observations show that tracheal epithelial polarization, septate junction formation, intraluminal chitin cable degradation and chitin deposition in the mature aECM taenidial folds are not dependent on tpr.

\subsubsection{Tracheal-prostasin is required for attachment of the tracheal cuticle to tracheal cells and contributes to degradation and localization of Dumpy}

The previous chapter shows, that $\mathrm{Tpr}$ is not required for chitin synthesis, remodeling and organization during tracheal development. However, a role of Tpr in tracheal aECM remodeling and organization was nevertheless assumed, due to its expression during late tracheal development and specific localization to the tracheal aECM (chapter 2.2.3). Besides chitin, the mature tracheal aECM contains proteins and lipids and is defined by a specific stratified organization (see chapter 1.1.3 and Fig 1D-E) that may be affected by Tpr. Therefore, the ultrastructure of the mature tracheal aECM of tpr mutant embryos was analyzed by transmission electron microscopy.

Tracheal taenidial folds are present in mid-stage 17 tpr mutant embryos and show a wild-type-like stratified structure (Fig 41B). An outermost envelope layer (red arrows in Fig 41) and a subjacent procuticle layer are distinguishable in both, wildtype and tpr mutant trachea (Fig 41). As observed in other studies, the thin epicuticle layer in the tracheal cuticle is usually not detectable (Moussian et al. 2006a). In contrast to wild-type trachea, the taenidial folds of tpr mutant trachea are spatially separated from the underlying apical plasma membrane (red arrowhead; Fig 41B). While the mature tracheal aECM in wild-type trachea is attached to the apical plasma membrane (Fig 41A; see also Fig 13G), a relatively 

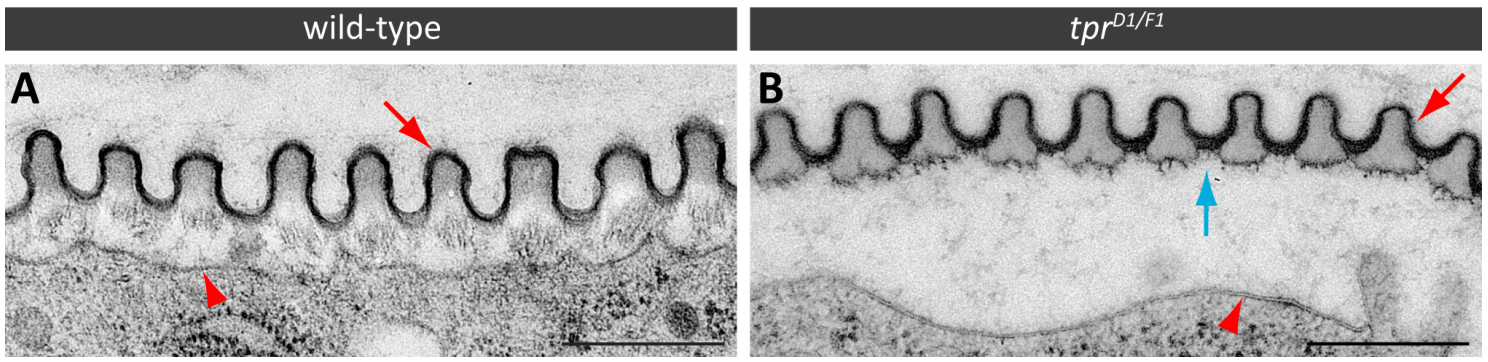

Figure 41: Tracheal-prostasin is required for attachment of the tracheal cuticle to apical plasma membranes

Transmission electron microscopy images of tracheal aECM in stage 17 wild-type (A) and $t p r^{D 1} / t p r^{F 1}$ mutant (B) embryos. Taenidial folds of wild-type (A) and tpr mutant (B) trachea contain an envelope layer (red arrows) and a subjacent procuticle layer. Wild-type taenidial folds are attached to the underlying apical plasma membrane (red arrow in A). In contrast, the taenidial folds of tpr mutant trachea (B) are detached from the apical plasma membrane (red arrowhead in B) and the procuticle layer is frequently disrupted (blue arrow in B). Scale bars correspond to $1 \mu \mathrm{m}$.

large gap between apical membrane and taenidial folds is observable in tpr mutant trachea (Fig 41B). Furthermore, the procuticle layer in tpr mutant trachea is frequently disrupted between individual taenidial folds (blue arrow in Fig 41B) and only the envelope layer shows a continuous structure (red arrow in Fig 41B). These results indicate that Tpr is required for attachment of the mature tracheal aECM to tracheal apical membranes and suggest that Tpr plays a role in formation of a continuous procuticle layer.

Since localization of chitin strands in the taenidial folds is not dependent on tpr (Fig 40B"'), defects in the mature tracheal aECM of tpr mutant embryos may be caused by deregulation of aECM proteins. Therefore, intraluminal degradation and localization of Dpy during aECM maturation in the tracheal system of tpr mutant embryos was analyzed by stainings of Dpy::YFP and chitin. Dpy::YFP is integrated into the intraluminal aECM cable of stage 16 tpr mutant trachea and is enriched in the core and peripheral region of the aECM cable (Fig 42A-A"), as observed in wildtype-like trachea of $d p y:$ YFP embryos (Fig 15A-A"). In early-stage 17 tpr mutant embryos, wild-type-like contraction of the intraluminal chitin and Dpy::YFP cable is visible and the mature tracheal aECM starts to form (Fig 42B-C"; compare with dpy::YFP trachea in Fig 15 B-B"). However, in contrast to wild-type embryos, the intraluminal Dpy::YFP cable is not fully degraded in mid-stage 17 tpr mutant embryos (Fig 42D-D"; compare with dpy: YFP trachea in Fig 15C-C"). This is 
evident by disorganized segments of the Dpy::YFP cable that remain in the tracheal lumen of tpr mutants after degradation of intraluminal chitin (Fig 42DD"). Furthermore, Dpy:YFP shows no co-localization with chitin of the mature tracheal aECM in mid-stage 17 tpr mutant trachea (Fig 42D-D"), unlike Dpy::YFP in wild-type-like trachea (Fig 15C-C"). These observations show that Tpr contributes to degradation of intraluminal Dpy and is required for integration of Dpy into the mature tracheal aECM.

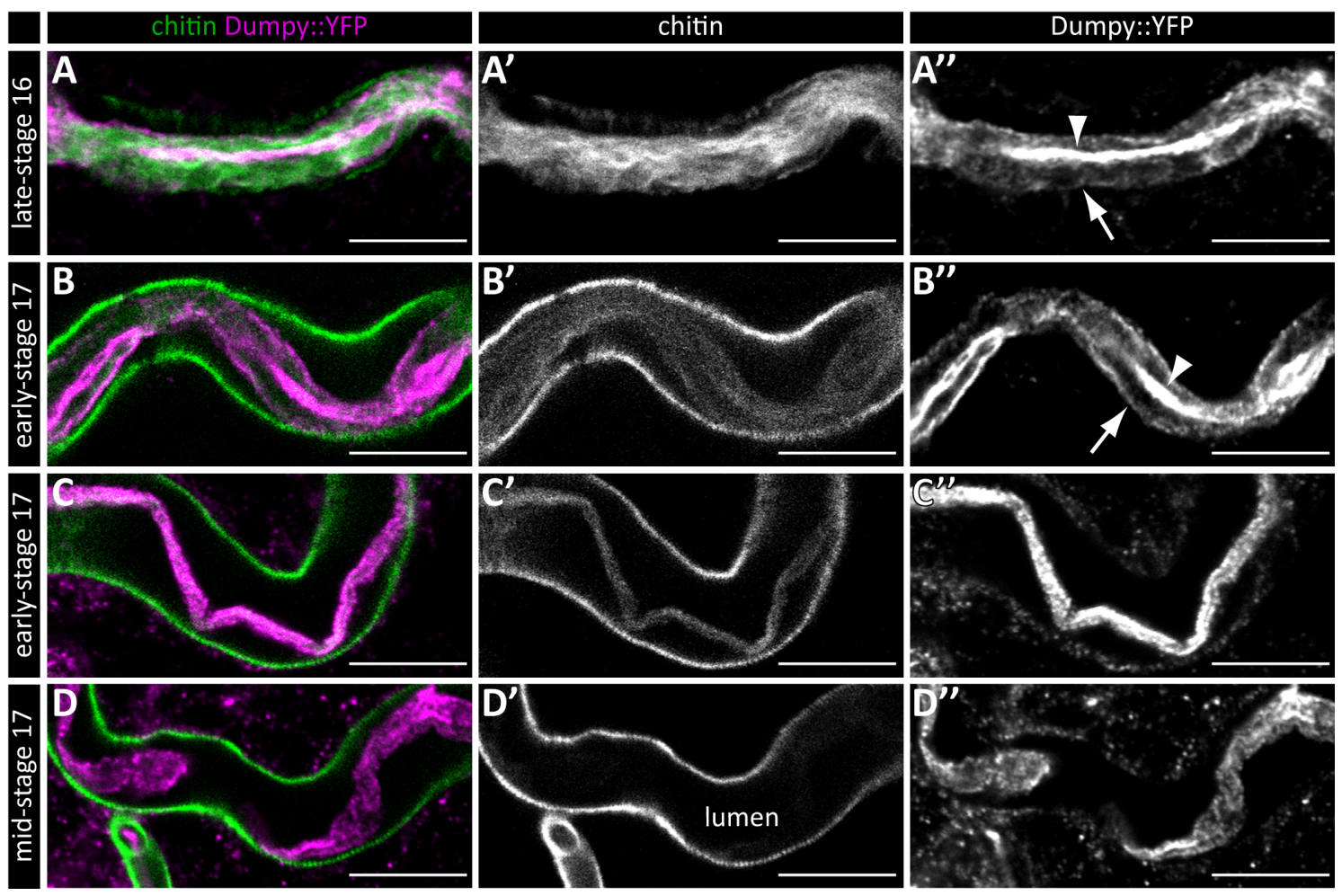

Figure 42: Tracheal-prostasin contributes to intraluminal Dumpy degradation and is required for Dumpy localization in the tracheal cuticle

Confocal LSM images of tracheal dorsal trunks of $t p r^{D 1}$, dpy::YFP/tpr ${ }^{F 1}$, dpy::YFP mutant embryos at late-stage 16 (A-A"), early-stage 17 (B-C") and mid-stage 17 (D-D") that were stained with FITC-labeled CBP (chitin; green) and anti-GFP antibodies (magenta). The intraluminal Dpy:YFP cable in late-stage 16 tpr mutant trachea is formed normally, localizing to the core (arrowhead) and peripheral region (arrow) of the intraluminal chitin cable (A-A"), as observed in $d p y:$ YFP/dpy::YFP embryos (Fig 15A-A"). The intraluminal aECM cable contracts during early-stage 17 (B-C") and intraluminal chitin is removed from the lumen until mid-stage 17 (D'). In contrast to wild-type-like dpy::YFP embryos, degradation of intraluminal Dpy::YFP in mid-stage 17 tpr mutant embryos is incomplete and Dpy::YFP does not co-localize with chitin of the mature aECM (D, D"; compare with dpy::YFP trachea in Fig 15C-C"). Images in B-C" show different stages of intraluminal aECM cable contraction during early-stage 17. Scale bars correspond to $10 \mu \mathrm{m}$. 


\subsubsection{Human prostasin is functionally homologous to Tracheal-prostasin}

Previous chapters show that tpr function in the tracheal system is similar to a subset of $N p$ functions: $N p$ and tpr are both essential for tracheal LC (chapters 2.1.2 and 2.2.2), for intraluminal Dpy degradation (chapters 2.1.8 and 2.2.5) and morphogenesis of the tracheal cuticle (chapters 2.1.6 and 2.2.5). However, phenotypes are more severe in $N p$ mutant than in tpr mutant embryos and unlike $t p r, N p$ is also required for tracheal transepithelial barrier function maintenance (chapters 2.1.5) and detachment of the intraluminal aECM cable from tracheal cells (chapters 2.1.8 and 2.1.9). Furthermore, Np was identified as a functional homolog of human matriptase (chapter 2.1.16). An essential interaction partner and downstream target of vertebrate matriptase is the serine protease prostasin (Miller and List 2013). In chapter 2.2.1, Tpr was shown to share high sequence similarities with vertebrate prostasin. These observations suggest that Tpr may be functionally homologous to vertebrate prostasin and that interactions of vertebrate matriptase and prostasin may be conserved between $\mathrm{Np}$ and $\mathrm{Tpr}$ in $D$. melanogaster. To analyze functional similarities of human prostasin with Tpr, UAS-prostasin responder strains were established for tissue specific expression of human prostasin in tpr mutant embryos.

Pan-tracheal expression of human prostasin in the tracheal system of tpr mutant embryos does not rescue lack of tracheal gas filling (Fig 43A, E). However, antibody stainings of prostasin in these embryos reveal that prostasin localizes to the cytoplasm of tracheal cells and is not secreted into the tracheal lumen (Fig 43C-C"), unlike Tpr which predominately localizes to the tracheal lumen wild-type-like embryos (Fig 38C-D"). Activity of prostasin and its translocation to the plasma membrane are regulated by HAI-2 in vertebrates, similar to HAI-2 mediated inhibition and secretion of matriptase (Lee et al. 2018; Friis et al. 2014). Previous experiments in the $D$. melanogaster embryo also show that secretion of human matriptase into the tracheal lumen depends on its human co-factor HAI-2 (chapter 2.1.16). Therefore, human prostasin and HAI-2 were co-expressed in the tracheal system of tpr mutant embryos to possibly induce apical secretion of prostasin. Strikingly, these embryos show wild-type-like tracheal gas filling (Fig 43B, E) and the co-expression of HAI-2 mediates secretion of prostasin into the tracheal lumen (Fig 43D-D"). The rescue of tracheal gas filling is most likely due to the function of prostasin in the tracheal lumen, since tracheal expression of only HAI-2 does not 


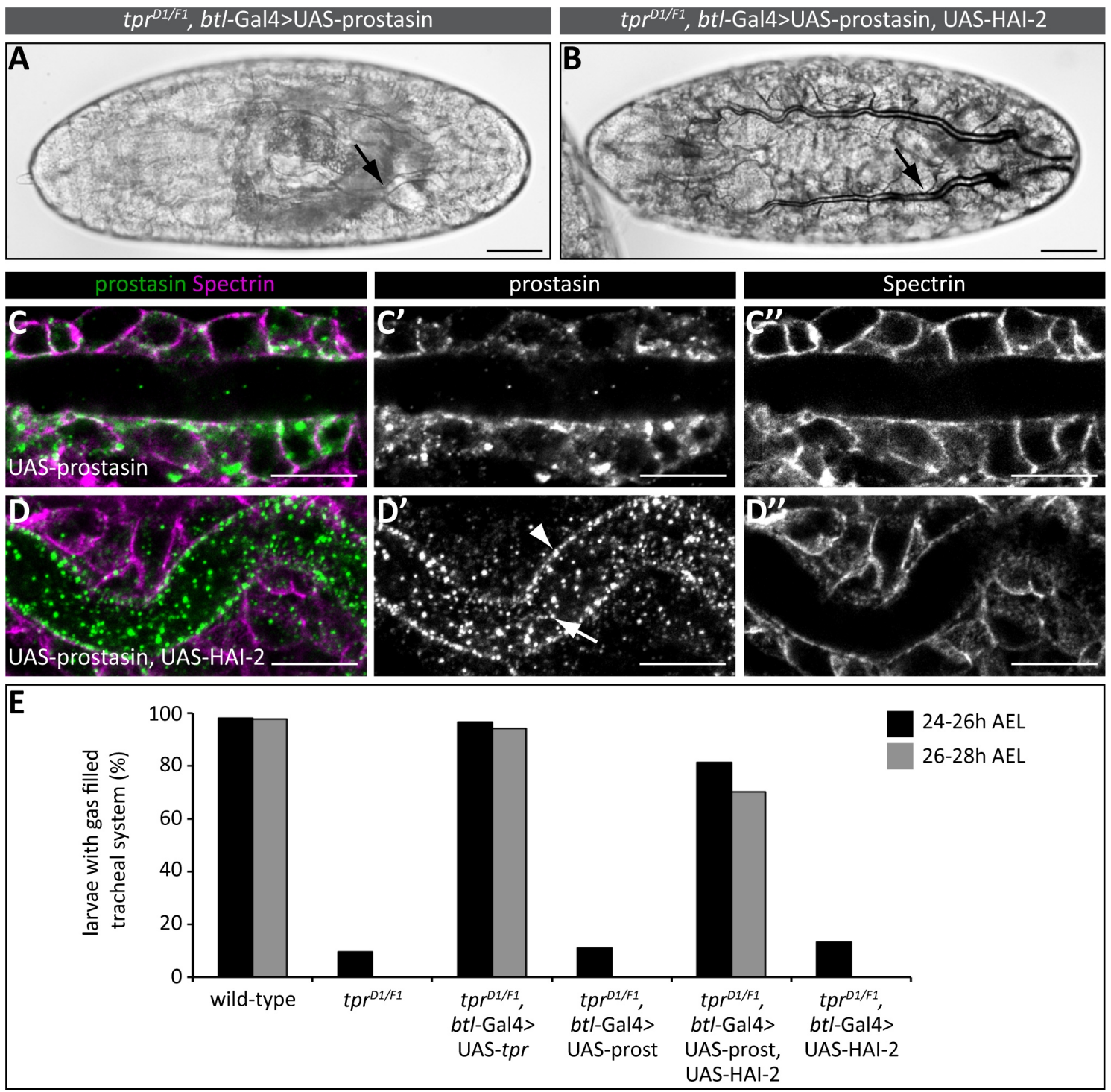

Figure 43: Human prostasin is functionally homologous to Tracheal-prostasin

(A-B) Bright field light microscopic images of late-stage $17 t_{p r}^{D 1}, b t l$-Gal4 / tpr ${ }^{F 1}$,UASprostasin (A) and $t p r^{D 1}, b t l$-Gal4 / tpr ${ }^{F 1}$,UAS-prostasin ; UAS-HAI-2 (B) embryos. Tracheal expression of prostasin in tpr mutant embryos does not rescue tracheal gas filling (arrow in A) but tracheal co-expression of prostasin and HAI-2 leads to LC of the tracheal system in tpr mutant embryos (B). Scale bars correspond to $50 \mu \mathrm{m}$. (C-D") Confocal LSM images of tracheal dorsal trunks of stage $16 t_{p r}^{D 1}, b t l$-Gal4 / tpr $r^{F 1}$,UAS-prostasin (C-C") and tpr ${ }^{D 1}$, btlGal4 / $t p r^{F 1}$,UAS-prostasin ; UAS-HAI-2 (D-D") embryos that were stained with antiprostasin (green) and anti-Spectrin (magenta) antibodies. Tracheal expression of HAI-2 mediates localization of prostasin to the tracheal lumen (arrow in $\mathrm{D}^{\prime}$ ) and apical plasma membranes (arrowhead in D'). Scale bars correspond to $10 \mu \mathrm{m}$. (E) Quantification of larvae with tracheal gas filling for wild-type, $t p r^{D 1} / t p r^{F 1}$ mutants and $t p r^{D 1} / t p r^{F 1}$ mutants with btlGal4. Driven expression of indicated UAS-transgenes. For each genotype, n=150. Larvae were analyzed immediately after hatching at $24-26$ hours AEL and at $26-28$ hours AEL. Data for larvae of wild-type, tpr mutants, and tpr mutants with tracheal expression of tpr were previously presented in Figure $36 \mathrm{~F}$ and are shown again here, to be compared with the rescue of tracheal LC by prostasin and HAI-2 expression. 
rescue lack of LC in tpr mutant embryos (Fig 43E). These rescue experiments suggest that human prostasin is functionally homologous to D. melanogaster Tpr.

It is well-established that matriptase mediates activation of the prostasin zymogen by proteolytic cleavage in vertebrates (Miller and List 2013). This suggests, that $\mathrm{Np}$ might similarly activate the Tpr zymogen by proteolytic cleavage in $D$. melanogaster. To analyze Tpr zymogen activation in vitro, carboxy-terminal Flagtagged Tpr (Tpr::Flag) and zymogen-locked Tpr (Tpr ${ }^{\mathrm{ZL}}:$ :Flag) with a mutated proteolytic activation site (Fig 44A) were expressed in cultured D. melanogaster cells and purified from cell culture supernatants via the Flag-tags. Tpr::Flag and $\operatorname{Tpr}^{\mathrm{ZL}}::$ Flag were then incubated with purified Np::Strep or matriptase::Strep and samples were analyzed by SDS-PAGE followed by western blotting and stainings
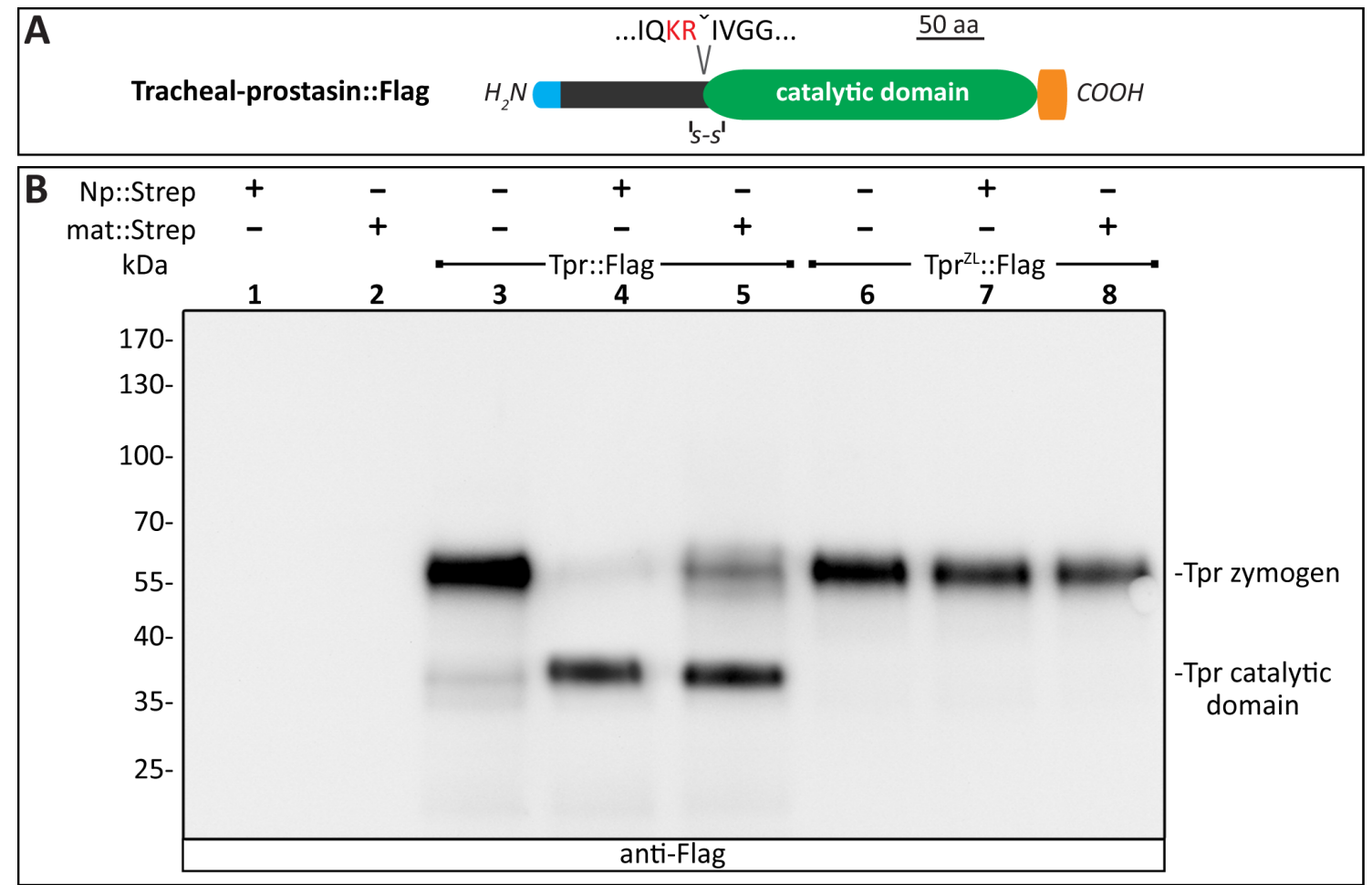

Figure 44: The Tracheal-prostasin zymogen is activated by Notopleural and matriptase

(A) Schema of Tpr::Flag protein. The signal peptide (blue), catalytic serine protease domain green), Flag-tag (orange) conserved disulphide bridge between catalytic domain and prodomain (-S-S-) and the zymogen activation cleavage site (V) are indicated. Lysine and arginine of the zymogen activation site (red letters) were substituted with alanine in zymogen locked $\mathrm{Tpr}^{\mathrm{ZL}}::$ Flag. (B) Biochemical assays in which purified Tpr::Flag or $\mathrm{Tpr}^{\mathrm{ZL}}::$ Flag were incubated with purified Np::Strep or purified matriptase::Strep were analyzed by SDS-PAGE followed by western blotting and stainings with anti-Flag antibodies. Tpr::Flag is detectable as a fragment of $\sim 60 \mathrm{kDa}$ (lane 3) that is processed to a fragment of $\sim 38 \mathrm{kDa}$ by incubation with purified Np::Strep or purified matriptase::Strep (lanes 4-5). Proteolytic processing occurs at the zymogen activation site, since zymogen locked $\mathrm{Tpr}^{\mathrm{ZL}}::$ Flag is resistant to cleavage by Np::Strep and matriptase::Strep (lanes 6-8). 
with anti-Flag antibodies. Purified Tpr::Flag is mainly detectable as a fragment of $\sim 60 \mathrm{kDa}$ and a faint fragment of $\sim 38 \mathrm{kDa}$ (lane 3 in Figure 44B), that may correspond to the Tpr zymogen (predicted mass: $45 \mathrm{kDa}$ ) and catalytic domain (predicted mass: $30 \mathrm{kDa}$ ). This indicates that purified Tpr::Flag is mainly present in its zymogen form, which is supported by the observation that $\mathrm{Tpr}^{\mathrm{ZL}}::$ Flag is only detectable as a fragment of $\sim 60 \mathrm{kDa}$, corresponding to the Tpr zymogen (lane 6 in Fig 44B). When incubated with Np::Strep or matriptase::Strep, proteolytic cleavage of Tpr::Flag is detectable by highly increased levels of the cleaved $\sim 38 \mathrm{kDa}$ catalytic domain and decreased levels of the $\sim 60 \mathrm{kDa}$ Tpr zymogen (lanes 4-5 in Fig 44B). Since no cleavage of zymogen locked $\mathrm{Tpr}^{\mathrm{ZL}}::$ Flag by Np::Strep or matriptase::Strep is detectable (lanes 7-8 in Fig 44B), cleavage of Tpr::Flag likely occurs at the conserved zymogen activation motif that is mutated in $\mathrm{Tpr}^{\mathrm{ZL}}::$ Flag and thereby provides cleavage resistance to $\mathrm{Np}$ and matriptase.

These results indicate that the Tpr zymogen is activated via proteolytic cleavage by $\mathrm{Np}$ and matriptase in vitro, and represents another common substrate of $\mathrm{Np}$ and matriptase, besides Pio (chapter 2.1.17). In addition to the functionally homologous roles of prostasin and Tpr during tracheal development, these observations suggest that activation of the prostasin and Tpr zymogens are similarly mediated by $\mathrm{Np}$ and matriptase, respectively. 


\subsection{Functional analysis of lumens interrupted in embryogenesis}

A detailed functional analysis of lint during embryogenesis was carried out with similar methods as previously used for functional analyses of $N p$ and $C G 4386$ in chapters 2.1 and 2.2. CRISPR/Cas9 technology was used to generate lint loss-offunction alleles and lint mutant embryos were analyzed by in vivo observations and immunohistochemical stainings of aECM components and epithelial markers. Expression pattern of lint transcripts was analyzed by whole-mount in situ RNAhybridization stainings and localization of Lint protein was determined by using embryos with endogenous expression of a Lint::YFP fusion protein. Finally, doublemutant embryos for lint and $N p$ were generated via CRISPR/Cas9 technology and analyzed to determine the functional relationship between $\mathrm{Np}$ and Lint. In a first step, an in silico analysis of Lint was carried out.

\subsection{1 lumens interrupted encodes a type II transmembrane serine protease with sequence similarities to Notopleural}

The gene lint (also known as filzig) is located $5 \mathrm{~kb}$ downstream of $N p$ on the second chromosome. Three protein isoforms are encoded by lint: Lint-B and Lint-C (Fig 45A) have the predicted protein domain organizations of TTSPs, while Lint-D lacks a catalytic protease domain (Gramates et al. 2017). Lint-C differs from Lint-B in only 19 amino acids in the extracellular stem region. All Lint isoforms share predicted domain structures with a cytoplasmic amino-terminal tail, a transmembrane domain and an extracellular stem that contains a DedD-like domain and an Atrophin-1-like domain. Only Lint-B and Lint-C have a predicted carboxy-terminal trypsin-like serine protease catalytic domain with a conserved zymogen activation cleavage site and a disulphide bridge that links the catalytic domain to the stem region (Fig 45A). A comparison of Lint-B and Lint-C with human proteins by BLASTP (Altschul et al. 1997) shows that the catalytic domain of Lint shares sequence similarities with the TTSP polyserase (also known as TMPRSS9) which belongs to the matriptase protein family. However, a BLASTP comparison of human polyserase with $D$. melanogaster proteins does not identify Lint as the most similar protein and the same is true when comparing polyserase homologs from other vertebrate species with $D$. melanogaster proteins by BLASTP. 
Although a putative vertebrate ortholog of Lint could not be identified, the catalytic domains of $\mathrm{Np}$ and Lint are highly similar, sharing a sequence identity of $44 \%$ (Fig 45B). Thus, lint encodes a TTSP with similarities to Np.

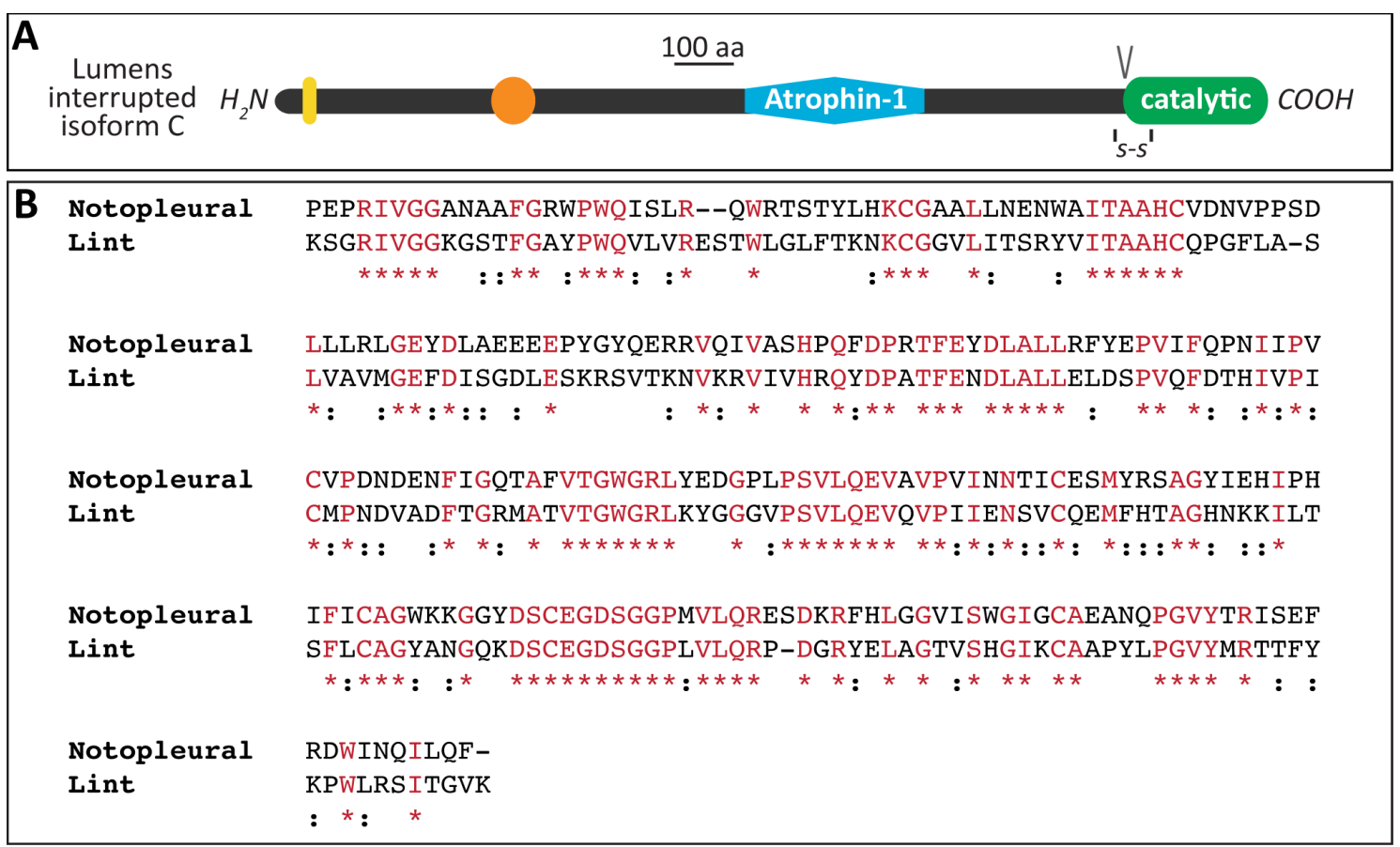

Figure 45: lumens interrupted encodes a type II transmembrane serine protease with sequence similarities to Notopleural

(A) Schemata of the protein domain organization of Lint isoform C. The transmembrane domain (yellow), cell division protein DedD-like domain (orange), Atrophin-1-like domain (blue) and the catalytic serine protease domain (green) are shown. The conserved disulphide bridge (-S-S-) between the catalytic domain and stem region as well as the zymogen activation cleavage site (V) are indicated. (B) Sequence alignment of the catalytic domains of $\mathrm{Np}$ and Lint by Clustal omega (Sievers et al. 2011) reveals a $44 \%$ sequence identity (red; asterisks) and additional $20 \%$ sequence similarity (colon).

\subsection{2 lumens interrupted is essential for tracheal liquid clearance and transepithelial barrier function}

For a detailed functional analysis of lint during embryogenesis, lint loss-of-function alleles were generated using CRISPR/Cas9 technology (Fig 46A-5C). Two independently generated lint alleles were obtained that cause frame-shifts in the lint ORF followed by premature translation stops (Fig 46B-C). The lint $t^{C 1}$ and lint ${ }^{K 7}$ alleles fail to complement each other as well as the Df(2R)BSC271 chromosome in which the chromosomal region 44F12-45A12 is deleted, including lint. Homozygous 
$\operatorname{lin} t^{C 1}$ or $\operatorname{lin} t^{K 7}$ mutant embryos and transheterozygous lint $t^{C 1} / \operatorname{lin} t^{K 7}$ mutant embryos lack tracheal gas filling in late-stage 17 of embryogenesis (Fig 46D). Furthermore, lint mutant embryos do not hatch as larvae. However, these embryos pass through all embryonic developmental stages and show movements during stage 17, indicating that lethality occurs at the end of embryogenesis. To test whether lack of tracheal gas filling in lint mutant embryos is caused by the CRISPR/Cas9 mediated mutations in lint, tracheal system specific rescue experiments using the UAS/Gal4

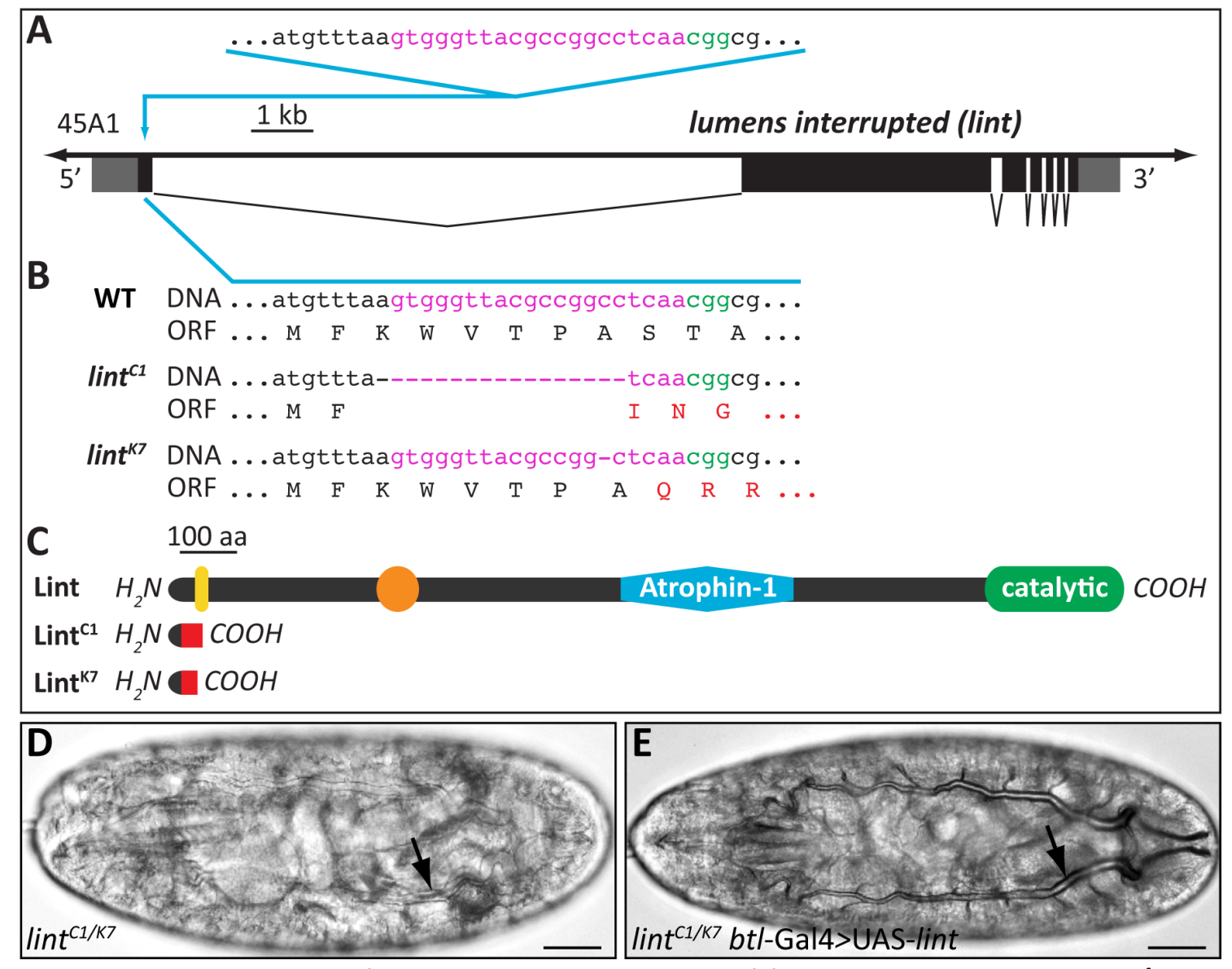

Figure 46: Generation of lumens interrupted loss-of-function alleles by CRISPR/Cas9mediated mutagenesis

(A-C) Schema of CRISPR/Cas9-mediated mutagenesis to generate frame shift mutations in the 5' region of the lint ORF. Physical map of lint genomic region shows the sgRNA recognition site (magenta letters) with PAM (green letters), translated DNA (black boxes) and UTRs (grey boxes; A). Generated DNA indels cause ORF frame shifts in lint ${ }^{C 1}$ and lint $t^{K 7}$ mutant alleles (B). Frame shifts result in expression of truncated Lint proteins (C). Transmembrane domain (yellow), cell division protein DedD-like domain (orange), Atrophin-1-like domain (blue), catalytic serine protease domain (green) and regions with frame-shifted amino acid sequences (red) are indicated (C). (D-E) Bright field light microscopic images of stage 17 lint ${ }^{C 1} / l i n t^{K 7}$ (D) and UAS-lint; lint ${ }^{C 1}$, btl-Gal4/lint ${ }^{K 7}$ (E) mutant embryos. Homozygous lint mutant embryos show no tracheal LC (arrow in D), while homozygous lint mutant embryos with tracheal expression of lint display normal tracheal LC (arrow in E). Scale bars correspond to $50 \mu \mathrm{m}$. 
system were carried out. An UAS-lint responder strain was established and used for ectopic pan-tracheal expression of Lint in lint mutant embryos. Ectopic tracheal expression of Lint facilitates tracheal gas filling in lint mutant embryos in latestage 17 (Fig 46E), indicating that lack of tracheal LC in lint mutant embryos is indeed caused by knock-out of lint. However, lint mutant embryos with tracheal expression of Lint still do not hatch as larvae and die at the end of embryogenesis. These experiments show that lint is essential for gas filling of the tracheal system and also has essential functions in other tissues, apart from the tracheal system.

As mentioned earlier, establishment of a continuous tracheal network and transepithelial barrier function are prerequisites for tracheal gas filling (chapter 1.1.3; Behr et al. 2003). To visualize tracheal network formation, the intraluminal chitin cable of stage 16 lint mutant embryos was stained, revealing a continuous wild-type-like tracheal system in these embryos (Fig 47A; compare with wild-type in Fig 9A). Next, tracheal transepithelial barrier function in early-stage 17 lint

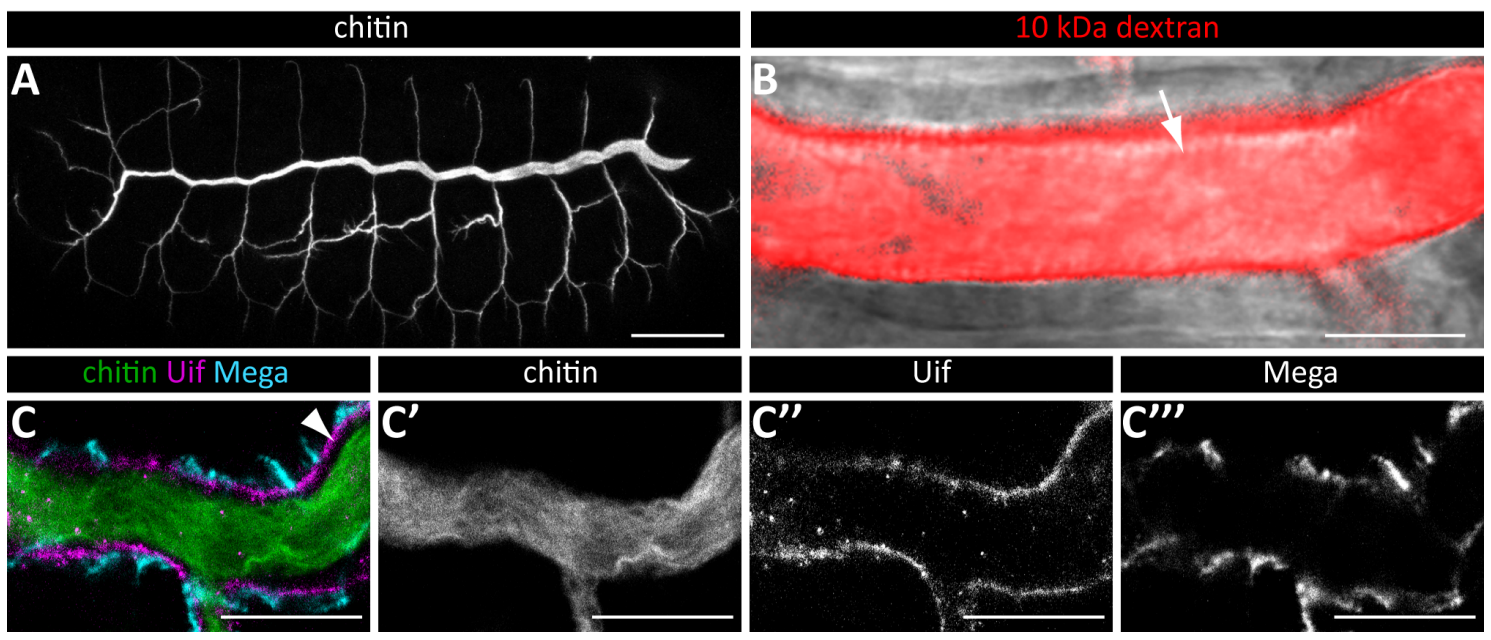

Figure 47: Lumens interrupted is required for tracheal transepithelial barrier function but not for tracheal network formation, epithelial polarization and septate junction formation

(A) Confocal LSM z-stack projection image of a stage 16 lint $t^{C 1} / l i n t^{K 7}$ mutant embryo that was stained with FITC-labeled CBP (chitin). Tracheal network formation of lint mutant embryos is wild-type-like (compare with Fig 9A). Scale bar corresponds to $50 \mu \mathrm{m}$. (B) In vivo LSM confocal image of a tracheal dorsal trunks of a stage 17 lint ${ }^{C 1} /$ lint ${ }^{K 7}$ mutant embryo after Texas Red-labeled $10 \mathrm{kDa}$ dextran injection into the haemocoel. Texas Red dextran (red) is detectable in the dorsal trunk lumen (arrow in B) indicating lack of transepithelial barrier function. Scale bar corresponds to $10 \mu \mathrm{m}$. (C-C'") Confocal LSM image of a tracheal dorsal trunk of a stage $16 \operatorname{lin} t^{C l} / \operatorname{lin} t^{K 7}$ mutant embryo that was stained with FITC-labeled CBP (chitin; green), anti-Uif (magenta) and anti-Mega (cyan) antibodies. The intraluminal chitin cable is formed normally and localization of Uif and Mega is wildtype-like (compare with Fig 10E-E"). A wild-type-like gap between intraluminal chitin cable and apical plasma membrane is observable (arrowhead). Scale bars correspond to $10 \mu \mathrm{m}$. 
mutant embryos was tested by injection of Texas Red-labeled $10 \mathrm{kDa}$ dextran into their haemocoel. Diffusion of the dye-labeled $10 \mathrm{kDa}$ dextran into the tracheal lumen of lint mutant embryos indicates lack of transepithelial barrier function in these embryos (Fig 47B). To test whether this phenotype is accompanied by lack of septate junction formation or loss of epithelial polarization, the apical membrane marker Uif, septate junction core component Mega and chitin were stained in lint mutant embryos. Formation of the intraluminal chitin cable, localization of Mega and localization of Uif are wild-type-like in stage 16 lint mutant trachea (Fig 47CC'"; compare with wild-type in Fig 10E-E"). These results demonstrate that lint is required for tracheal gas filling and function of the tracheal transepithelial barrier but not for formation of septate junctions and epithelial polarization in the tracheal system, as shown for $N p$ (chapters 2.1.2 and 2.1.4). However, while $N p$ mutant embryos display aberrant attachment of the intraluminal chitin cable to tracheal apical cell membranes (Fig 10F-F", H-H"), lint mutant embryos show a wild-typelike gap between the chitin cable and apical plasma membranes (arrowhead in Fig 47C; compare with wild-type in Fig 10E, G).

\subsubsection{Lumens interrupted is expressed in embryonic ectodermal tissues and localizes to the tracheal lumen in late embryogenesis}

To determine the developmental stages and embryonic tissues in which function of Lint might be essential, expression pattern of lint transcripts and localization of the Lint protein during embryogenesis were analyzed. RNA in situ hybridization stainings of wild-type embryos with a DIG-labeled lint antisense probe were performed to visualize the lint transcript expression pattern. Expression of lint is first detectable in the pharynx, foregut and posterior spiracles of stage 13 embryos (Fig 48A). During subsequent stages 14 to 17, lint expression is also detectable in the epidermis, hindgut and tracheal system (Fig 48B-D). Notably, expression of lint in the tracheal system starts in posterior segments of the stage 14 dorsal trunk (Fig 48B), is detectable in the whole tracheal system in stage 16 embryos (Fig 48C) and increases until stage 17 (Fig 48D). This analysis shows that lint is expressed in several ectodermal tissues in a developmental stage specific manner, with the highest expression levels during late embryogenesis. For analysis of Lint localization during embryogenesis, a lint::YFP strain was used that carries a YFP 


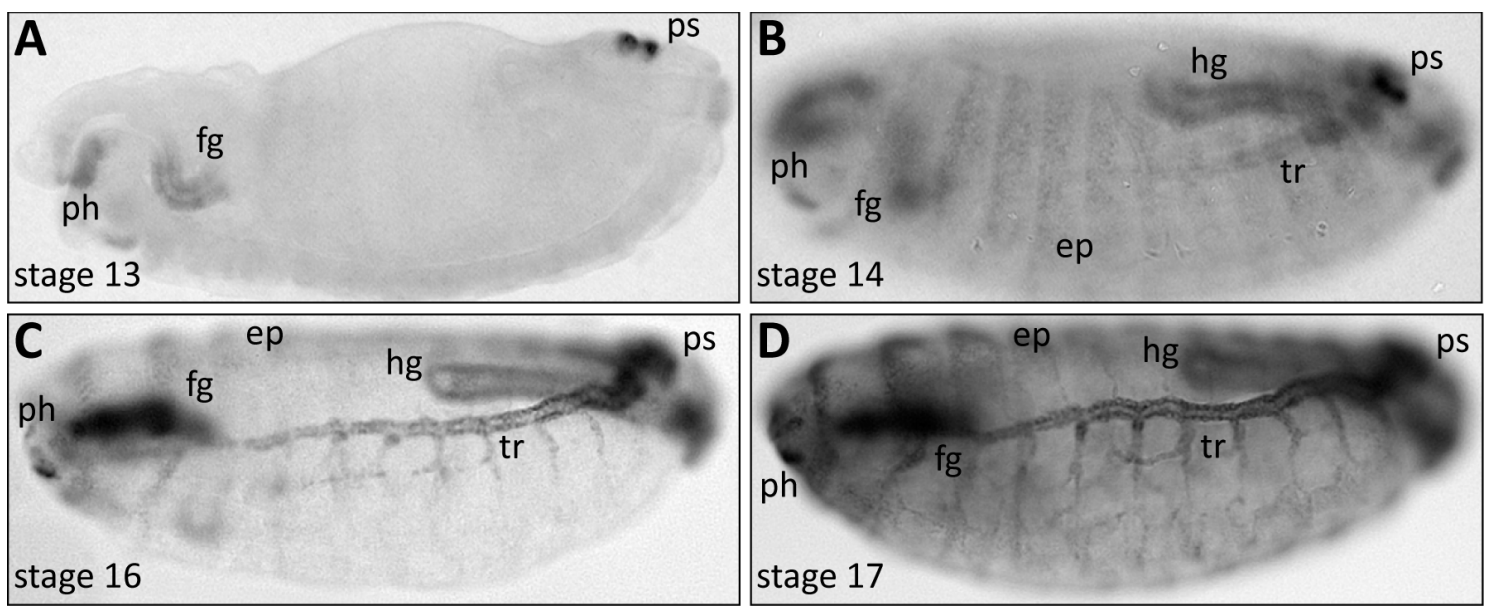

Figure 48: Expression of lumens interrupted during embryogenesis

Bright field light microscopic images of whole-mount in situ RNA hybridization stainings of wild-type embryos with a DIG-labeled lint antisense probe. Transcripts of lint are first detectable during stage 13 in the pharynx (ph), foregut (fg), and posterior spiracles (ps; A). During stages 14, 16 and 17, lint expression is also detectable in the hindgut (hg), epidermis (ep), and tracheal system (tr; B-D).

transgene insertion in the first intron of lint (Fig 49H; Lye et al. 2014). The YFP encoding sequence is flanked by a splice acceptor and a splice donor site and thereby integrated into the lint mRNA (Fig 49H), resulting in insertion of YFP into the Lint protein at the amino-terminal end of the extracellular stem region. Stainings of lint::YFP embryos with anti-GFP antibodies reveal a spatial expression pattern of Lint::YFP that corresponds to the expression pattern of lint transcripts. Lint::YFP is detectable in the posterior spiracles during stages 13 to 17 (Fig 49A-D). During stages 15 to 17, Lint::YFP is also detectable in the pharynx, foregut, epidermis, hindgut and tracheal system (Fig 49C-D). For analysis of subcellular Lint::YFP localization, lint::YFP embryos were stained with anti-GFP and anti-Spectrin antibodies. Lint::YFP localizes to the cytoplasm and apical membranes (arrowhead in Fig 49E') of tracheal cells in stage 16 embryos (Fig 49 EE"). This tracheal localization pattern changes during subsequent development and Lint::YFP localizes to apical cell membranes (arrowhead in Fig 49F') and the intraluminal aECM (arrow in Fig 49F') in the tracheal system of stage 17 embryos (Fig 49F-F"). In the hindgut, Lint::YFP is detectable in the cytoplasm and at apical plasma membranes during stage 17 (Fig 49G-G"). Thus, Lint localizes to the cytoplasm, apical plasma membrane, or aECM in a tissue and developmental stage specific manner. 

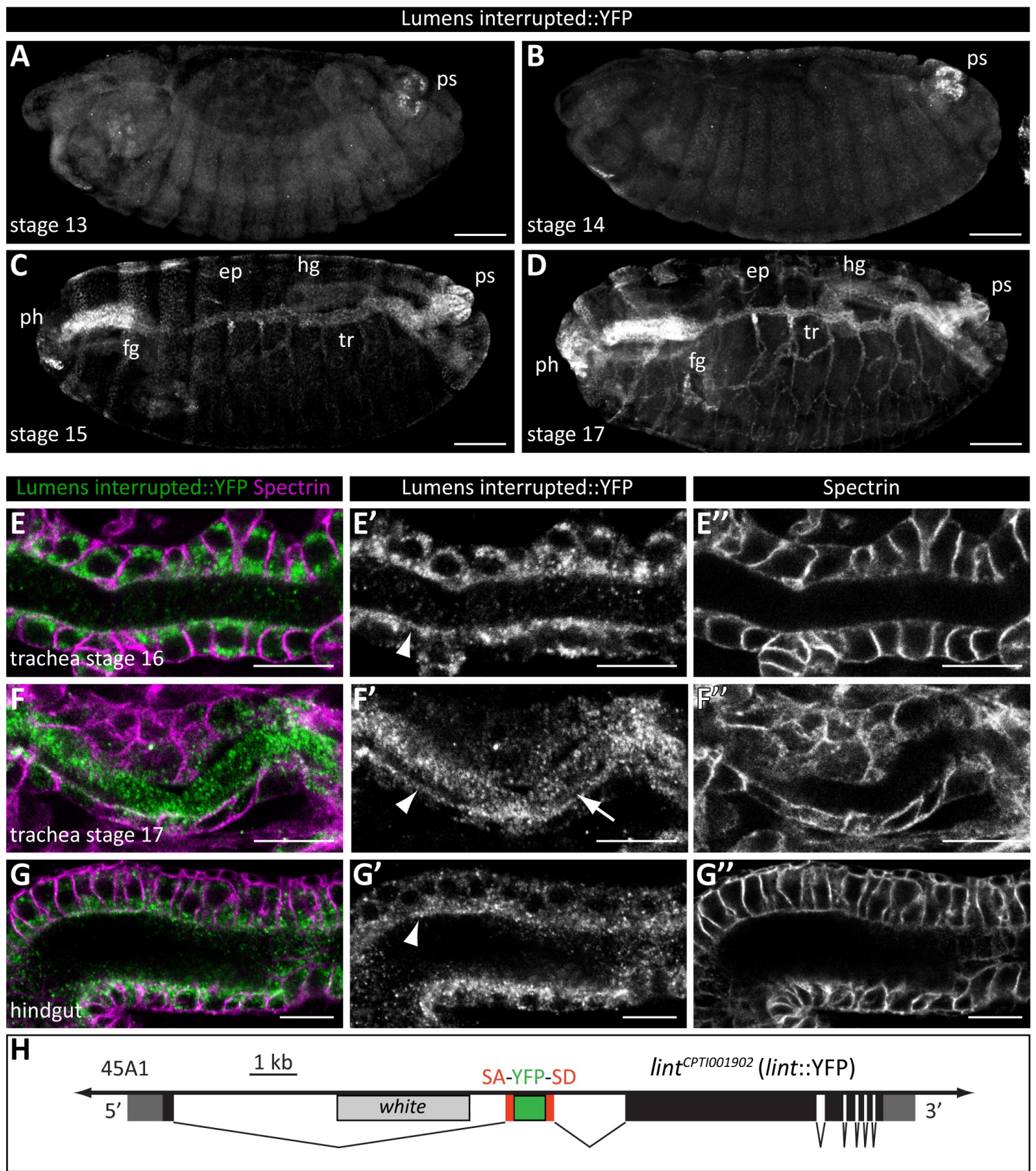

\section{Figure 49: Localization of Lumens interrupted during embryogenesis}

(A-G") Confocal LSM images of whole-mount anti-GFP (A-D) or anti-GFP and anti-Spectrin (E-G") antibody stainings of lint::YFP embryos at stage 13 (A), 14 (B), 15 (C), 16 (E-E") or 17 (D, F-G"). Lint::YFP is detectable in the posterior spiracles (ps), pharynx (ph), foregut (fg), epidermis (ep), hindgut (hg) and tracheal system (tr) in a developmental stage dependent manner (A-D). Images of tracheal dorsal trunks (E-F") and hindgut (G-G") of lint::YFP embryos show localization of Lint::YFP (green) and Spectrin (magenta). Lint::YFP localizes to the cytoplasm and apical cell membranes (arrowheads) in stage 16 trachea (E$\left.\mathrm{E}^{\prime \prime}\right)$ and stage 17 hindgut (G-G"). In stage 17 trachea, Lint::YFP is detectable in the intraluminal aECM (arrow) and at apical cell membranes (F-F"). Scale bars correspond to

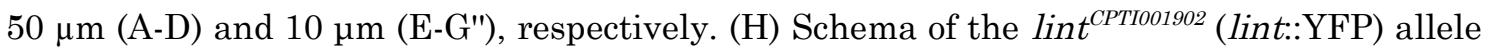
(Lye et al. 2014). Genomic region of lint shows UTRs (grey boxes), translated DNA (black boxes), white marker gene and the YFP encoding sequence (green box) flanked by splice acceptor (SA) and splice donor (SD) sites. 


\subsubsection{Lumens interrupted is required for tracheal intraluminal Dumpy degradation}

\section{and taenidial folds organization}

Localization of Lint to the intraluminal tracheal aECM coincides with intraluminal aECM cable degradation and tracheal cuticle formation during early stage 17 (chapter 2.3.3). Therefore, the role of Lint in degradation of the intraluminal aECM cable components Dpy and chitin, and in mature aECM taenidial folds formation was analyzed. Stainings of Dpy::YFP and chitin in lint mutant embryos reveal wild-type-like integration of Dpy into the chitin cable during stage 16 (Fig 50A-A"; compare with wild-type in Fig 15A-A"). Localization of Dpy::YFP at the core and margin of the intraluminal cable can be distinguished (arrowhead and arrow in Fig $50 \mathrm{~A} ")$. During early-stage 17 , the intraluminal aECM cable contracts and chitin

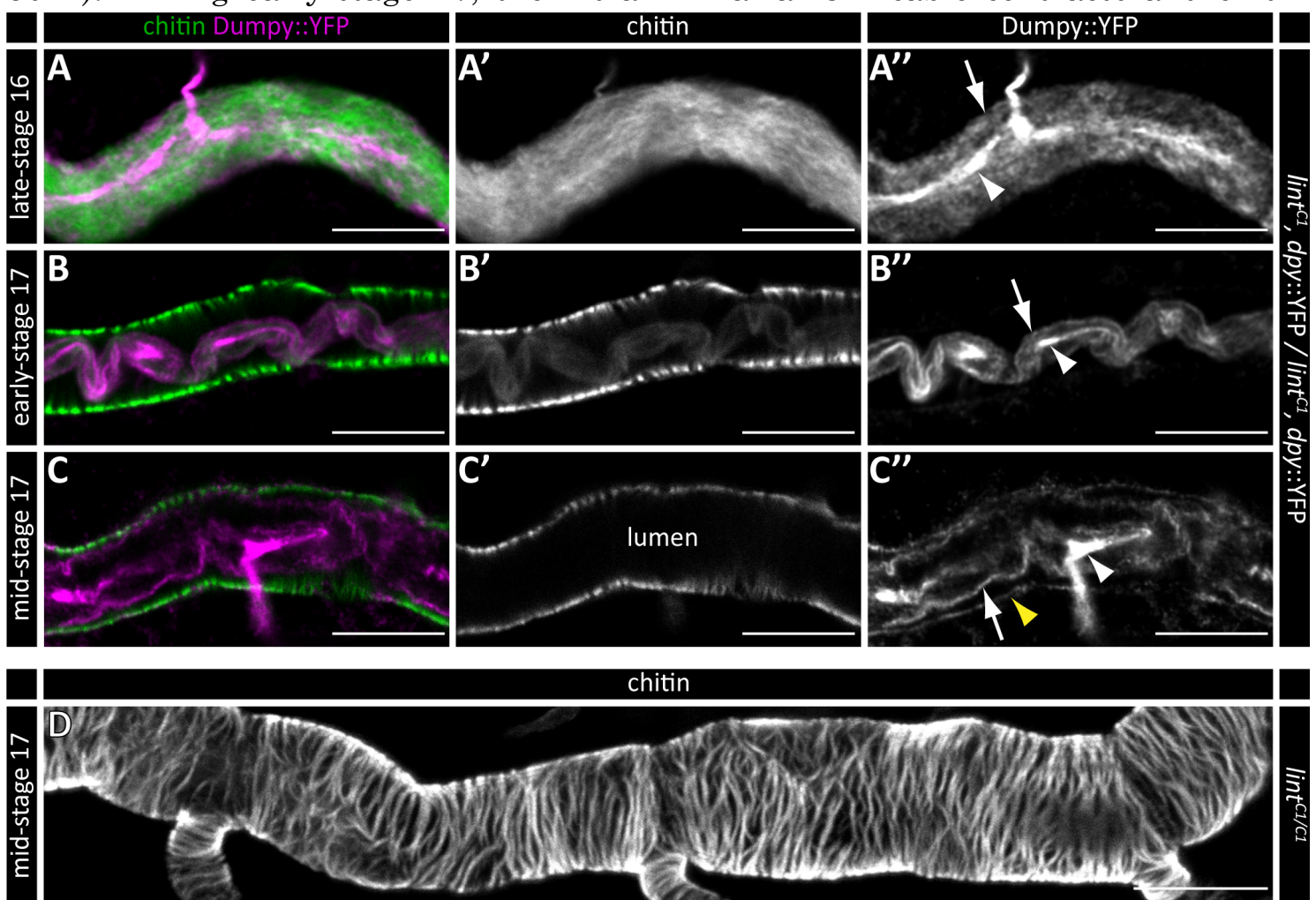

Figure 50: Lumens interrupted is required for tracheal intraluminal Dumpy degradation and taenidial folds organization

Confocal LSM images of dorsal trunks of lint ${ }^{C 1}$,dpy::YFP/ lint ${ }^{C 1}$, dpy::YFP mutant embryos at late-stage 16 (A-A"), early-stage 17 (B-B") and mid-stage 17 (C-D) stained with FITClabeled CBP (chitin; green) and anti-GFP antibodies (magenta). Intraluminal Dpy::YFP localizes at a central core (white arrowheads) and a peripheral "shell" layer (arrows). The intraluminal Dpy::YFP and chitin cable contract during early-stage 17 (B-B"). While the Dpy::YFP cable is not degraded, the chitin cable is normally removed from the lumen until mid-stage 17 (C-C"). In this stage, Dpy::YFP also localizes to the mature tracheal aECM (yellow arrowhead in $\left.\mathrm{C}^{\prime \prime}\right)$. Mid-stage 17 chitin strands show aberrant misorganization, similar to $N p$ mutant trachea (D; compare with wild-type and $N p$ mutant in Fig 13C-D). Scale bars correspond to $10 \mu \mathrm{m}$. 
depositions of the mature tracheal aECM start to form in lint mutant trachea (Fig 50B-B"), as observed in wild-type-like trachea (Fig 15B-B"). Strikingly, mid-stage 17 lint mutant embryos lack clearance of intraluminal Dpy::YFP, while showing normal completion of intraluminal chitin degradation (Fig 50C-C"). Similar to $N p$ mutant embryos (Fig 15F-G"), localization of Dpy::YFP at a core and peripheral region in the tracheal lumen is still detectable in mid-stage 17 lint mutant trachea (white arrowhead and arrow in Fig 50C"). In contrast to the contracted intraluminal aECM cable in early-stage 17 (Fig 50B-B"), the Dpy::YFP cable in mid-stage 17 lint mutant trachea is again expanded across the axial luminal width (Fig 50C-C"). Dpy::YFP is also integrated into the lint mutant tracheal mature aECM (yellow arrowhead in Fig 50C"), as observed in wild-type-like and $N p$ mutant embryos (Fig 15C", F"', G"). Interestingly, chitin strands of the lint mutant mature tracheal aECM are highly misorganized, showing no parallel organization and frequent intersections (Fig 50D), similar to what has been observed in $N p$ mutant trachea (Fig 13D; compare with wild-type in Fig 13C). However, unlike $N p$ mutant embryos, lint mutants display no formation of aECM bulges and instead show a relatively straight boundary of the tracheal lumen (Fig 50C-C', D; compare with $N p$ mutant trachea in Fig 15G-G").

These results indicate that Lint is required for tracheal intraluminal Dpy degradation and taenidial folds organization. Np is similarly required for taenidial folds formation and intraluminal Dpy degradation (chapters 2.1.6 and 2.1.8), but in addition, Np also mediates contraction of the intraluminal aECM cable and release of Pio from apical tracheal membranes during late-stage 16 to early-stage 17 (chapters 2.1.8 and 2.1.10). While Lint is not required for intraluminal aECM cable contraction (Fig 50B-B"), it seemed likely that shedding of Pio from apical membranes might also be dependent on Lint, due to the otherwise very similar phenotypes of lint and $N p$ mutant trachea. Therefore, tracheal subcellular localization of mCherry::Pio in lint mutant embryos was analyzed by stainings of chitin and mCherry::Pio. Surprisingly, mCherry::Pio shows wild-type-like localization during aECM maturation in lint mutant trachea (Fig 51; compare with wild-type in Fig 20B-D"). Intraluminal localization of mCherry::Pio (arrows in Fig 51A", B") and localization of mCherry::Pio to tracheal apical cell membranes (arrowheads in Fig 51A", B") are observable in lint mutant trachea during latestage 16 and early-stage 17, as observed in wild-type-like trachea (Fig 20B-C"). 
Furthermore, mCherry::Pio is normally removed from the tracheal lumen of lint mutant embryos until mid-stage 17 (Fig 51C-C"). These observations show that Lint is not required for release of Pio from tracheal apical membranes.
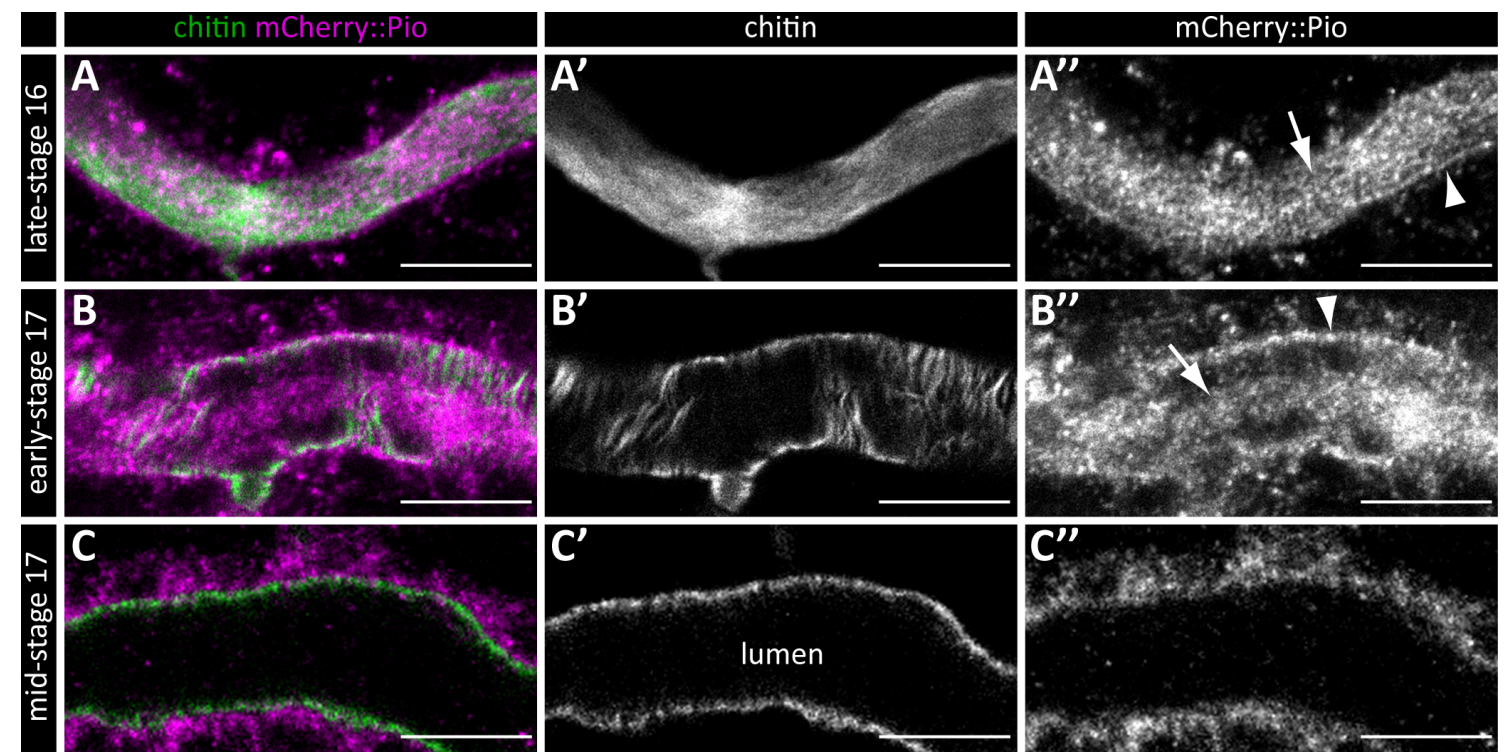

Figure 51: Lumens interrupted does not affect release of Piopio into the tracheal lumen

Confocal LSM images of dorsal trunks of lint $t^{C 1}$,mCherry::pio / lint $t^{C 1}$, mCherry::pio mutant embryos at late-stage 16 (A-A"), early-stage 17 (B-B") and mid-stage 17 (C-C") stained with FITC-labeled CBP (chitin; green) and anti-mCherry antibodies (magenta). Localization of mCherry::Pio is wild-type-like in all shown stages (compare with wild-type in Fig 20B-D"). In late-stage 16 and early-stage 17, mCherry::Pio is detectable in the lumen (arrows) and at apical cell membranes (arrowheads). Scale bars correspond to $10 \mu \mathrm{m}$.

\subsubsection{Genetic interactions of Lumens interrupted and Notopleural}

Previous chapters demonstrate that lint and $N p$ are both essential for tracheal LC (chapters 2.1.2 and 2.3.2), transepithelial barrier function (chapters 2.1.5 and 2.3.2), intraluminal Dpy degradation (chapters 2.1.8 and 2.3.4) and organization of the aECM taenidial folds (chapters 2.1.6 and 2.3.4). However, only $N p$ mediates intraluminal aECM cable contraction and shedding of Pio from tracheal apical membranes (chapters 2.1 .8 and 2.1.10). It was also shown that $N p$ regulates attachment of tracheal apical membranes to the aECM (chapter 2.1.9). To test whether lint has a similar role in tracheal cell - aECM attachment regulation, localizations of the apical membrane marker Uif and chitin were analyzed in lint mutant trachea during aECM maturation. The chitin strands of the lint mutant tracheal cuticle form at the apical surface of tracheal cells during early-stage 17 (Fig 52A-A"). Detachments of chitin strands from the apical membrane are not 

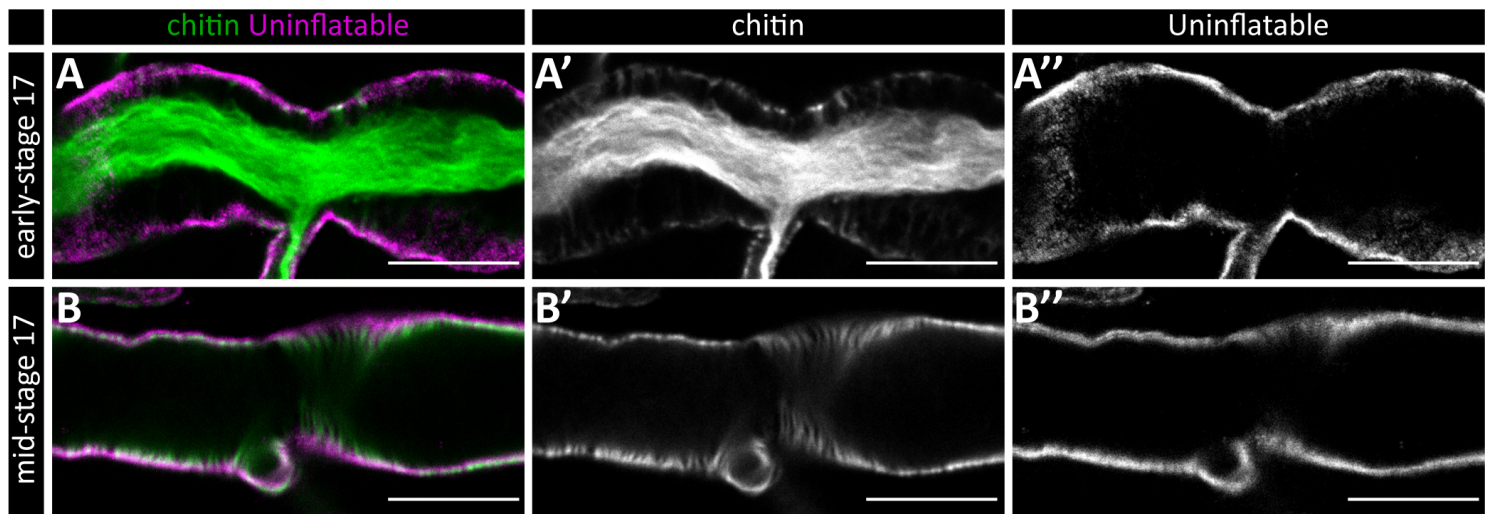

Figure 52: Attachment of chitin to tracheal cells is not regulated by Lumens interrupted

Confocal LSM images of dorsal trunks of lint $t^{C 1} /$ int $t^{K 7}$ mutant embryos at early-stage 17 (AA") and mid-stage 17 (B-B") stained with CBP (chitin; green) and anti-Uif antibodies (magenta). The chitin strands of the tracheal cuticle form on the apical surface of tracheal cells and in separation from the intraluminal chitin cable (A-A"), as seen in wild-type trachea (Fig 16A-B"). Scale bars correspond to $10 \mu \mathrm{m}$.

observable and apical cellular membranes and associated chitin strands are spatially separated from the intraluminal chitin cable (Fig 52A-A"), as seen in wildtype embryos (Fig 16A-B"). In mid-stage 17 lint mutant embryos, the misorganized chitin strands line the apical surface of tracheal cells and again, no detachments are detectable (Fig 52B-B"). Previous observations also show that lint mutant trachea display a wild-type-like gap between chitin cable and apical membranes during stage 16 (Fig 47C). These observations suggest that Lint does not regulate cell - chitin attachments during maturation of the tracheal cuticle. Therefore, regulation of cell - aECM attachment is yet another function that is mediated by Np (chapters 2.1.8 - 2.1.9) but not by Lint.

To further elucidate the partially overlapping functions of $\mathrm{Np}$ and Lint and to identify putative epistatic effects, a $N p$, lint double mutant allele was generated using CRISPR/Cas9 technology (Fig 53A-B). The obtained $N p^{22 F}$, lint ${ }^{22 F}$ allele contains a $9 \mathrm{bp}$ insertion in the $N p$ ORF and a 1 bp insertion in the lint ORF causing frame-shifts and premature translation stops in both genes (Fig 53B). Furthermore, the $N p^{22 F}$, lint $t^{22 F}$ allele fails to complement the previously generated $N p^{P 6}, \quad N p^{C 2}$, lint $t^{C 1}$ and lint $t^{K 7}$ alleles as well as the deficiency chromosome $\mathrm{Df}(2 \mathrm{R}) \mathrm{BSC} 271$ in which the chromosomal region 44F12-45A12 including $N p$ and lint, is deleted. As observed for $N p$ mutant and lint mutant embryos, $N p$, lint double mutant embryos lack gas filling of the tracheal system during late 


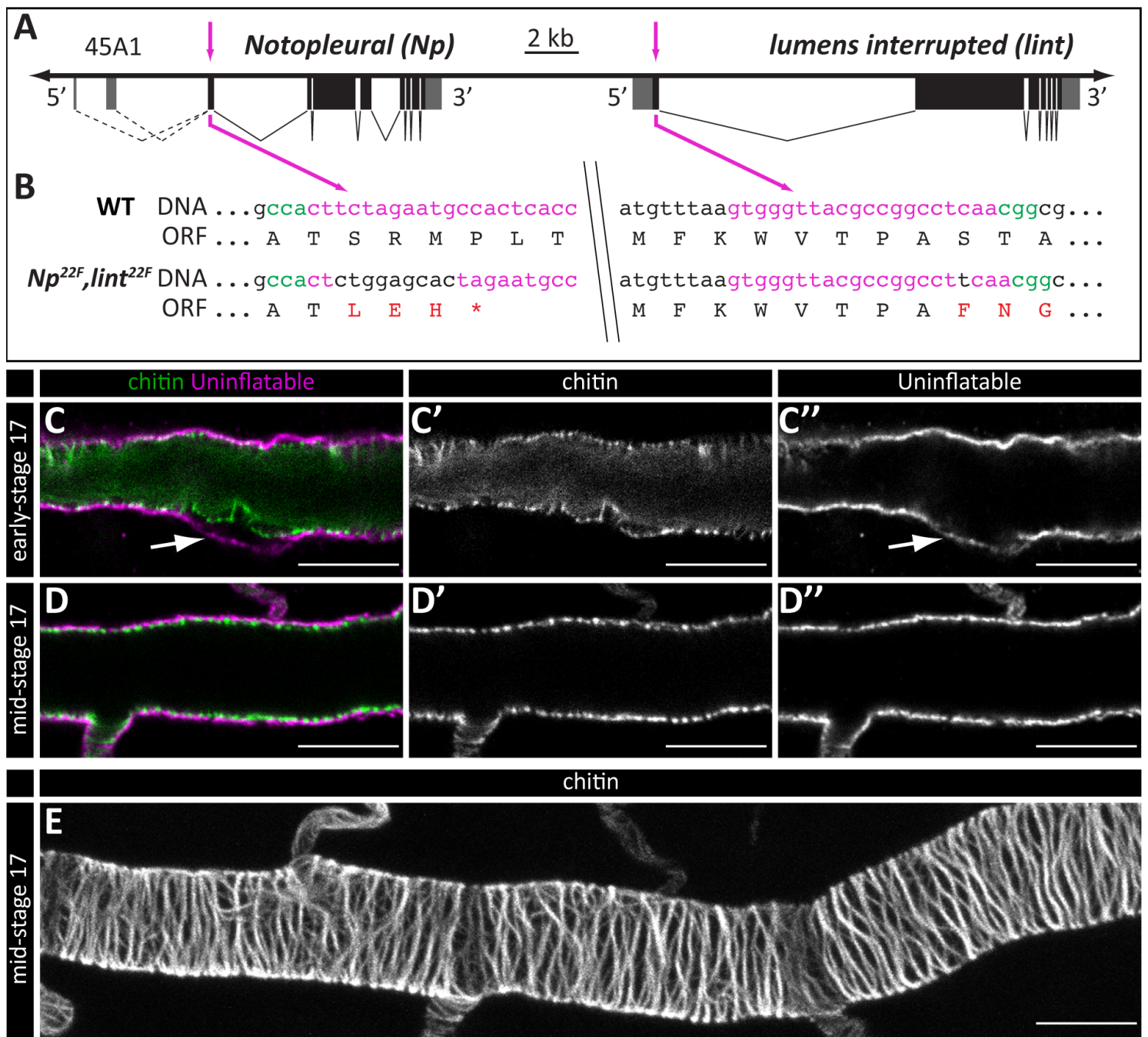

Figure 53: Generation and analysis of $N p$, lint double mutant embryos

(A-B) Schema of CRISPR/Cas9-mediated mutagenesis to simultaneously generate frame shift mutations in the 5' regions of the $N p$ ORF and lint ORF. Physical map of the $N p$ - lint genomic region shows sgRNA target sites (magenta arrows), translated DNA (black boxes) and UTRs (grey boxes; A). Generated DNA indels cause ORF frame shifts in the $N p^{22 F}$, lint $t^{22 F}$ double mutant allele (B). Target sequences of sgRNAs (magenta letters) and PAMs (green letters) are indicated (B). (C-E) Confocal LSM images of dorsal trunks of $N p^{22 F}$, $\operatorname{lin} t^{22 F} / N^{22 F}$, lint ${ }^{22 F}$ double mutant embryos at early-stage 17 (C-C") and mid-stage 17 (D-E) stained with FITC-labeled CBP (chitin; green) and anti-Uif antibodies (magenta). Embryos lack contraction of the tracheal intraluminal chitin cable during early-stage 17 (C-C"). Apical membranes are aberrantly detached from the forming mature aECM chitin strands in randomly distributed areas (arrows in $\left.\mathrm{C}, \mathrm{C}^{\prime \prime}\right)$. While no formation of aECM bulges is observable (D-E; compare with Np mutant trachea in Fig 16 E-F"), chitin strands of the mature aECM are highly misorganized in mid-stage 17 trachea (E). Scale bars correspond to $10 \mu \mathrm{m}$. 
embryogenesis and do not hatch as larvae. To analyze tracheal cell - aECM attachment and aECM maturation, stainings of chitin and the apical membrane marker Uif in homozygous $N p^{22 F}$, lint $t^{22 F}$ embryos were performed. Early-stage 17 $N p$, lint double mutant trachea show most aspects of the $N p$ mutant tracheal phenotype (Fig 53C-C"). As observed in $N p$ mutant trachea, the intraluminal chitin cable in early-stage $17 \mathrm{~Np}$, lint double mutant trachea does not contract during degradation and instead remains attached to the forming mature aECM and tracheal apical cell membranes in most areas (Fig 53C-C"; compare with $N p$ mutant in Fig 16D-E"). Furthermore, randomly distributed areas of the tracheal apical cell membranes in early-stage $17 \mathrm{~Np}$, lint double mutant trachea are detached from the forming mature aECM that aberrantly localizes to the margin of the intraluminal chitin cable (arrows in Fig 53C-C"). The same phenotype is observable in late-stage $16 N p$ mutant trachea (Fig 16D-D"). During subsequent development of $N p$ mutant trachea, a second layer of misorganized chitin strands is formed at areas of detached apical membranes, which ultimately leads to the formation of rigid aECM bulges (chapter 2.1.9). Formation of such tracheal bulges is not observable in $N p$, lint double mutant trachea and the tracheal lumen displays a straight boundary (Fig 53D-E), as previously observed in lint mutant trachea (Fig 50C-D) and seen in wild-type trachea (Fig 16C-C"). However, midstage $17 \mathrm{~Np}$, lint double mutant trachea display misorganized chitin strands in the mature tracheal aECM, resembling the phenotypes of $N p$ mutant and lint mutant trachea (Fig 13D, Fig 50D).

These results indicate positive epistatic effects between lint and $N p$ mutant alleles since knock-out of lint suppresses formation of tracheal cuticle bulges that are usually caused by homozygous $N p$ mutant alleles (chapter 2.1.9). 


\section{Discussion}

The results of this thesis show that three serine proteases mediate tracheal aECM maturation and gas filling during $D$. melanogaster embryogenesis. The serine proteases Np, Lint and Tpr target the ZPD protein Dpy in the transient aECM of the tracheal lumen and facilitate its degradation. In addition, they regulate the formation, membrane-attachment and organization of the mature tracheal aECM cuticle. Unlike Tpr, $\mathrm{Np}$ and Lint are also required for function of the transepithelial barrier in the tracheal system. The obtained data furthermore suggest that $\mathrm{Np}$ has a central regulatory role in aECM maturation: Np activates the Tpr zymogen by proteolytic cleavage, mediates shedding of the ZPD protein Pio from apical tracheal membranes by proteolytic cleavage and also regulates formation and organization of the epidermal cuticle, including epidermal localization patterns of Dpy and Pio. A central role of $\mathrm{Np}$ in the initiation of aECM maturation is also supported by observed autocatalytic Np zymogen activation in vivo and in vitro. Rescue experiments identified the human TTSP matriptase and serine protease prostasin as functional homologs of $D$. melanogaster $\mathrm{Np}$ and $\mathrm{Tpr}$, respectively. Functional homology of $\mathrm{Np}$ and matriptase is based on a conserved substrate specificity, since matriptase cleaves the $D$. melanogaster proteins Pio and Tpr, as reported for Np. Finally, this thesis presents evidence that cleavage of ZPD proteins by matriptase homologs may be conserved across invertebrates and vertebrates since both, human matriptase and $D$. melanogaster $\mathrm{Np}$ mediate ectodomain shedding of the human ZPD containing TGF-B receptor type III by proteolytic cleavage in cultured cells. 


\subsection{Tracheal apical extracellular matrix maturation is mediated by three serine proteases}

The functions and expression patterns of $\mathrm{Np}$, Lint and Tpr that are reported in this thesis suggest that these three proteases interact during tracheal aECM maturation and might form a proteolytic cascade. $N p$ is expressed in tracheal cells during all stages of embryonic tracheal development, and therefore precedes expression of lint and tpr which are first detectable in the tracheal system during stages 14 and 15, respectively (chapters 2.1.3, 2.2.1 and 2.3.3). Np is deposited in the tracheal aECM upon expression and integrated into the intraluminal aECM cable during tracheal network formation in stage 14 (chapter 2.1.3). In contrast, localization of Tpr to the tracheal aECM is established in stage 16 and while Lint is present in tracheal cells during stage 16, its localization to the tracheal aECM is not detectable until early-stage 17 (chapters 2.2.3 and 2.3.3). This temporally staggered presence of Np, Tpr and Lint in the tracheal aECM coincides with their functions during aECM maturation. While all three proteases mediate similar and essential functions during stage 17 aECM maturation, $\mathrm{Np}$ also regulates the attachment of tracheal cells to the aECM during stage 16 (chapter 2.1.9). Furthermore, Np activates the Tpr zymogen by proteolytic cleavage in vitro, and functions of $\mathrm{Np}$ and Lint are highly similar without being redundant (chapters 2.1.6, 2.2.6 and 2.3.5). Taken together, these observations suggest a proteolytic cascade in which $\mathrm{Np}$ activates Lint and Tpr and thereby mediates tracheal aECM maturation.

\subsubsection{Non-cell autonomous initiation of tracheal apical extracellular matrix maturation by Notopleural}

As mentioned in the previous paragraph, functions of $\mathrm{Np}$ in tracheal aECM maturation precede the functions of Lint and Tpr. Np mediates detachment of the intraluminal aECM cable from tracheal apical cell membranes in mid-stage 16, leading to subsequent contraction of the aECM cable during its degradation in early-stage 17. Evidently, $N p$ mutant trachea display aberrant attachment of the aECM cable to apical plasma membranes during mid-stage 16 and following stages (chapter 2.1.9). In addition, $N p$ mutant trachea lack taenidial folds formation 
(chapter 2.1.6). These two phenotypes may be causally linked since formation and organization of the tracheal cuticle at the apical tracheal cell surface might be hampered by the attached intraluminal aECM cable in $N p$ mutant trachea. Indeed, Np-mediated detachment of the luminal aECM cable from apical membranes coincides with the onset of mature aECM taenidial folds formation which starts in mid-stage 16 with deposition of the hydrophobic cuticle envelope (Moussian et al. 2006a).

The mechanism by which $\mathrm{Np}$ mediates detachment of the tracheal intraluminal aECM cable from cellular membranes might require proteolytic ectodomain shedding of Pio. The ZPD protein Pio has been suggested to provide a link between cell membranes and the aECM in multiple studies due to its roles in tracheal cell intercalation and epidermis - cuticle attachment (Öztürk-Çolak et al. 2016a; Bökel et al. 2005; Jaźwińska et al. 2003). Np mediates the release of Pio from apical cell membranes into the tracheal lumen with the beginning of tracheal aECM maturation in mid-stage 16 to late-stage 16 (chapter 2.1.10). Furthermore, proteolytic processing of Pio in the embryo, in cultured cells and in biochemical assays is mediated by $\mathrm{Np}$, thus identifying Pio as a direct target of $\mathrm{Np}$ proteolysis (chapters 2.1.12 and 2.1.17). Due to these results, I propose a mechanisms in which $\mathrm{Np}$ regulates detachment of the intraluminal aECM cable from apical plasma membranes by proteolytic cleavage of Pio (Fig 54A-B) and thereby enables formation of the mature aECM taenidial folds. In this model, Pio links the apical plasma membranes to intraluminal Dpy by heterophilic binding of their ZPDs (Fig 54A). This link is then released via cleavage of Pio by Np (Fig 54B). The proposed interaction of Dpy and Pio is based on the observations that Dpy is localized at the peripheral region of the intraluminal aECM cable which is most proximal to the tracheal apical plasma membranes (Fig 54; Dong et al. 2014). Peripheral Dpy also remains attached to tracheal cells in the absence of Pio ectodomain shedding in $\mathrm{Np}$ mutant trachea (chapter 2.1.8). Furthermore, heterophilic polymerization of ECM proteins via ZPDs is well established and Pio and Dpy are both required for tracheal cell intercalations and epidermis - cuticle attachment, indicating interactions between Dpy and Pio (Bökel et al. 2005; Jovine et al. 2005; Jaźwińska et al. 2003; Wilkin et al. 2000). 


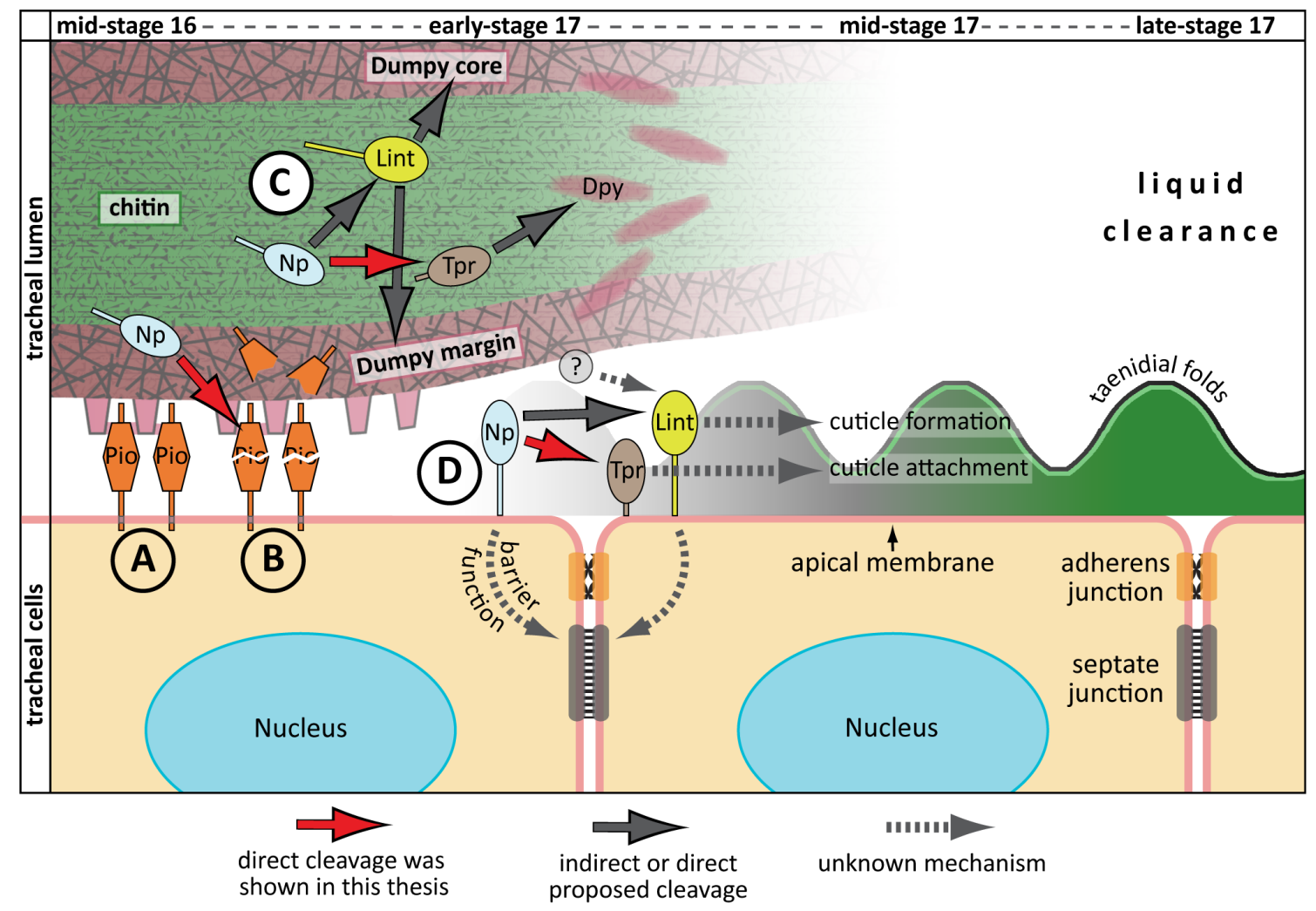

Figure 54: Functional model of the proposed proteolytic cascade which is initiated by Notopleural in the tracheal system

Autocatalytic zymogen activation of Np initiates aECM maturation in the tracheal system. (A) The intraluminal tracheal aECM cable which contains chitin and Dpy is linked to the tracheal cells by ZPD interactions of Dpy and Pio. Dpy fibrils are enriched at the core and margin of the aECM cable. (B) The link between intraluminal aECM cable and tracheal cells is released via proteolytic cleavage of the Pio ZPD by Np. The cleaved ectodomain of Pio is released into the tracheal lumen. (C) Np activates Lint and Tpr by proteolytic cleavage in the tracheal lumen. Activated Lint mediates disassembly of the macromolecular Dpy structures at the core and margin of the luminal aECM cable. Activated Tpr mediates degradation of the disassembled Dpy fragments. (D) Proteolytic activation of Lint and Tpr by $\mathrm{Np}$ at the tracheal cell surface mediates formation of the tracheal cuticle taenidial folds and their attachment to the underlying apical membrane. $\mathrm{Np}$ and Lint regulate transepithelial barrier function by an unknown mechanism which affects function of the septate junctions. Activation of Lint by an unknown protease might contribute to taenidial folds formation.

Notably, cleavage of the Pio ZPD at the Np protease cleavage site (pcs) is not exclusively mediated by $\mathrm{Np}$ in $D$. melanogaster embryos. This is apparent by basal levels of Pio processing at the $\mathrm{Np}$ pcs in $N p$ mutant embryos (chapter 2.1.12). However, this $\mathrm{Np}$ independent cleavage of the Pio ectodomain may be irrelevant for tracheal development since Pio is expressed in many ectodermal tissues during 
embryogenesis and shows the highest expression levels in the salivary glands (chapter 2.1.10). Similar to the observations in embryos, low levels of Pio cleavage at the $\mathrm{Np}$ pcs are detectable when Pio is expressed in cultured D. melanogaster cells (chapter 2.1.17). This might be due to basal Np expression in these cells. Indeed, expression data of $D$. melanogaster cell lines show that $N p$ is expressed at low levels in the cell lines used in this study (Brown et al. 2014).

The results of this thesis show that functions of $\mathrm{Np}$ in embryonic tracheal development are non-cell autonomous (chapter 2.1.13). The Np-mediated detachment of the intraluminal aECM cable from cellular membranes coincides with the non-cell autonomous spreading of $\mathrm{Np}$ throughout the tracheal system in mid- to late-stage 16 . Until early-stage $16, \mathrm{~Np}$ is anchored cell-autonomously in the tracheal intraluminal aECM cable and shows no diffusion throughout the tracheal lumen (chapter 2.1.13). Anchorage of $\mathrm{Np}$ may be mediated by its stem region which is predicted to be highly glycosylated, possibly enabling adhesion to ECM components as known from other glycosylated extracellular proteases (Boon et al. 2016; Steentoft et al. 2013; Van den Steen et al. 2001). Np that is detectable in the luminal tracheal aECM likely represents the $\mathrm{Np}$ ectodomain that was shedded from the apical membranes since ectodomain shedding is a common mechanism for the release of TTSPs into the extracellular space (Antalis et al. 2011). With the beginning of tracheal aECM maturation, $\mathrm{Np}$ is mobilized and non-cellautonomously localizes to the intraluminal aECM and apical cellular surface throughout the tracheal system (chapter 2.1.13). This observation suggests that $\mathrm{Np}$ is sequestered to the apical cell surface from the extracellular space with the onset of tracheal cuticle formation. Apical membrane-associated localization of $\mathrm{Np}$ therefore likely represents binding of the shedded $\mathrm{Np}$ ectodomain to components of the forming tracheal cuticle, rather than integration of $\mathrm{Np}$ into the plasma membrane. This hypothesis is supported by the observed localization of catalytically inactive $\mathrm{Np}^{\mathrm{S990A}}$ in the tracheal system. Homozygous $N p^{\mathrm{S990A}:: \text { Strep }}$ embryos show all aspects of the $N p$ mutant phenotype, including lack of taenidial folds formation. This lack of tracheal cuticle formation in $\mathrm{Np}^{S 990 A}$ ::Strep embryos is accompanied by absence of $\mathrm{Np}^{\mathrm{S} 990 \mathrm{~A}}$ from the apical surface of tracheal cells (chapter 2.1.14). In summary, these observations suggest that $\mathrm{Np}$ non-cell-autonomously initiates the first steps of tracheal aECM maturation and is integrated into the forming tracheal cuticle. 


\subsubsection{Luminal apical extracellular matrix clearance - proteolytic teamwork for Dumpy degradation}

After Np-mediated detachment of the tracheal intraluminal aECM cable from apical membranes in mid-stage 16, the luminal cable contracts and is degraded until mid-stage 17. Besides chitin and other ECM proteins, the gigantic modular 2,5 MDa ZPD protein Dpy is a major integral component of this transient luminal cable (Dong et al. 2014). Degradation of intraluminal Dpy during tracheal aECM maturation requires the proteolytic activity of the serine proteases $\mathrm{Np}$, Lint and Tpr, which might form a proteolytic cascade in the tracheal lumen. While no degradation of luminal Dpy is detectable in $N p$ mutant or lint mutant trachea, a large portion of luminal Dpy is degraded in the trachea of tpr mutant embryos (chapters 2.1.8, 2.2.5 and 2.3.4). The Dpy cable in tpr mutant trachea undergoes fragmentation during early-stage 17 and loses its sub-structuring into a central core and a peripheral region. Disassembly of the luminal Dpy fibrils and the macromolecular structure of the Dpy cable is therefore not dependent on Tpr. However, these disassembly steps are dependent on $\mathrm{Np}$ and Lint. As mentioned above, no degradation of the luminal Dpy cable or changes in its macromolecular structure are visible in $N p$ mutant or lint mutant trachea (chapters 2.1.8 and 2.3.4). This raises the possibility that $\mathrm{Np}$ and Lint form a proteolytic cascade. In this cascade, one protease would activate the other which then mediates Dpy degradation and therefore lack of each one of these proteases would similarly cause lack of Dpy degradation. Several observations suggest that $\mathrm{Np}$ is the initiator of this proteolytic cascade. First, Np localization in the tracheal lumen in stage 14 and following stages precedes the release of Lint into the tracheal lumen in earlystage 17 (chapters 2.1.3 and 2.3.3). Second, zymogen activation of $\mathrm{Np}$ in the $D$. melanogaster embryo and in cultured cells occurs in an autocatalytic manner (chapters 2.1.15 and 2.1.17). Third, the Np-mediated contraction of the intraluminal aECM cable occurs wild-type-like in lint mutant trachea, indicating $\mathrm{Np}$ activity in the absence of Lint (chapter 2.3.4). Furthermore, in vitro experiments in this thesis show that $\mathrm{Np}$ directly activates the Tpr zymogen by proteolytic cleavage (chapter 2.2.6). Based on these data, I propose that $\mathrm{Np}$, Lint and Tpr are parts of a proteolytic cascade that targets intraluminal Dpy. In this model, autocatalytically activated $\mathrm{Np}$ directly cleaves and activates the Tpr zymogen and indirectly or directly mediates Lint zymogen activation (Fig 54C). 
Disassembly of the macromolecular Dpy fibrils is then facilitated by Lint followed by Tpr mediated Dpy degradation. Notably, Np does not directly cleave Dpy in this model (Fig 54C), due to the observation that the Dpy cable is not disassembled in lint mutant embryos in which $\mathrm{Np}$ should be active.

\subsubsection{A proteolytic cascade drives formation of the tracheal cuticle taenidial folds}

The mature tracheal cuticle taenidial folds are formed during intraluminal aECM degradation from late-stage 16 to mid-stage 17 (Öztürk-Çolak et al. 2016a). The results of this thesis show that $\mathrm{Np}$, Lint and Tpr have essential functions in taenidial folds morphogenesis. Analyses of mutant phenotypes again support the hypothesis that $\mathrm{Np}$ activates Lint and Tpr in a proteolytic cascade.

Besides the lack of intraluminal Dpy degradation, $N p$ mutant, lint mutant and $N p$, lint double mutant trachea all display a similarly misorganized structure of the mature tracheal cuticle (chapters 2.1.6, 2.3.4 and 2.3.5). As discussed in the previous chapter, Np-mediated detachment of the luminal aECM cable from cellular membranes occurs wild-type-like in lint mutant trachea. Therefore I conclude, that Np-mediated aECM cable detachment might be a prerequisite, but not sufficient for taenidial folds organization which still requires Lint. However, functions of $\mathrm{Np}$ and Lint in taenidial folds morphogenesis are not redundant, since over-expression of Lint does not rescue the $N p$ mutant tracheal phenotype (chapter 2.1.16). As proposed for intraluminal Dpy degradation, these observations suggest that taenidial folds morphogenesis might be similarly facilitated by Np-mediated activation of Lint (Fig 54D). In contrast to $\mathrm{Np}$ and Lint, Tpr is not required for formation of the stratified tracheal cuticle and its taenidial folds patterning but mediates attachment of the tracheal cuticle to the underlying membrane (chapter 2.2.5). $\mathrm{Np}$ is similarly required for proper attachment of the tracheal cuticle chitin strands to apical membranes (chapter 2.1.9), while observations indicate that Lint has no role in cuticle - membrane attachment (chapter 2.2.2). Together with the observed cleavage of the $\mathrm{Tpr}$ zymogen by $\mathrm{Np}$ in vitro (chapter 2.2.6), these observations further support the previously proposed proteolytic cascade in which $\mathrm{Np}$ directly activates Tpr and mediates activation of Lint in parallel (Fig 54C-D). Intraluminal Dpy degradation and taenidial folds morphogenesis at the cellular 
surface would therefore be driven by the same proteolytic cascade, which is initiated by $\mathrm{Np}$ autoactivation.

The analysis of $\mathrm{Np}$, lint double mutant embryos also suggests an additional $\mathrm{Np}$ independent role of Lint in tracheal cuticle formation. $N p$ mutant trachea display rigid aECM bulges which aberrantly form in areas of membrane - cuticle detachments (chapter 2.1.9). This phenotype is not present in lint mutant trachea (chapter 2.3.4), and $N p$, lint double mutant trachea display areas of membrane cuticle detachment without formation of aECM bulges (chapter 2.3.5). Thus, Lint mediates the formation of aECM bulges in $N p$ mutant trachea. This may indicate a role of Lint in tracheal taenidial folds formation initiation which is independent of the proposed Lint zymogen activation by $\mathrm{Np}$ (Fig 54D). However, further experiments are needed to elucidate the roles and interactions of $\mathrm{Np}$ and Lint in tracheal aECM maturation. Ultrastructural analyses of the lint mutant and $N p$, lint double mutant tracheal cuticles would determine possible differences to the absence of taenidial folds organization in $N p$ mutant trachea. Most importantly, the ability of $\mathrm{Np}$ to activate the Lint zymogen has to be tested in cultured cells or in vitro to verify the proposed interaction. Interestingly, the observed role of Lint in embryonic tracheal aECM maturation is in agreement with a study that showed a role of Lint in luminal aECM clearance and tracheal cuticle formation in larval terminal branches (Rosa et al. 2018). Rosa et al. also showed that expression of Lint is activated by the transcription factor Ichor (Ich) but ectopic Lint expression in terminal tracheal branches does not rescue a knock-out of ich (Rosa et al. 2018). This might indicate that Ich also regulates expression of $\mathrm{Np}$, which would explain the lack of Lint function in absence of Ich.

While the presented results show that Np, Lint and Tpr regulate tracheal taenidial folds morphogenesis, detailed downstream molecular mechanisms and targets of the proposed proteolytic cascade remain to be explored. Tracheal taenidial folds morphogenesis might be intrinsically linked to intraluminal Dpy degradation but likely also involves other, currently unknown targets of $\mathrm{Np}$, Lint and Tpr. A promising downstream target candidate is the ZPD protein Tyn which was recently implicated in nanopatterning of the tracheal cuticle (Itakura et al. 2018). In addition, chitin-binding and -modifying proteins might be targeted by $\mathrm{Np}$, Lint or Tpr since they were shown to affect taenidial folds organization (Öztürk-Çolak et 
al. 2016a). Notably however, degradation and synthesis of chitin in the tracheal system seems to be independent of $\mathrm{Np}$, Lint and Tpr.

\subsubsection{Multiple functions of Notopleural, Lumens interrupted and Tracheal-prostasin facilitate tracheal liquid clearance}

$N p$, lint and tpr were initially identified in an RNAi screening for genes that are required for tracheal gas filling (Jaspers 2011). Generation and analyses of loss-offunction mutants confirm the essential roles of all three genes in tracheal LC (chapters 2.1.2, 2.2.2 and 2.3.2). The functions of $N p$ and lint even affect multiple physiological aspects of tracheal development that are known to be essential for tracheal gas filling. A prerequisite for $\mathrm{LC}$ is the establishment of the tracheal transepithelial barrier function which is provided by the septate junction complexes (Behr et al. 2003). Np mutant embryos display a novel phenotype in which the transepithelial barrier is initially established, but not maintained during stage 17 of embryogenesis while morphology of the septate junction complexes is wild-type-like (chapter 2.1.5). Similarly, lint mutant trachea lack transepithelial barrier function in stage 17 but show normal localization of septate junction markers (chapter 2.3.2). These similar phenotypes could again be due to a proteolytic cascade of $\mathrm{Np}$ and Lint as proposed in the previous chapters. However, neither Np nor Lint localize to the baso-lateral septate junctions and both are not required for epithelial polarization. It therefore remains elusive how regulation of the transepithelial barrier by $\mathrm{Np}$ and Lint is achieved. In addition to epithelial barrier function, the luminal clearance of transient aECM components and formation of the tracheal cuticle are well-known prerequisites for tracheal gas filling (Öztürk-Çolak et al. 2016a; Jaspers et al. 2014). As discussed in chapters 3.1.2 and 3.1.3, $\mathrm{Np}$ and Lint mediate luminal clearance of Dpy and tracheal taenidial folds morphogenesis and therefore facilitate tracheal gas filling by multiple functions.

Unlike $\mathrm{Np}$ and Lint, $\mathrm{Tpr}$ is not essential for tracheal transepithelial barrier function (chapter 2.2.4). Furthermore, some tpr mutant embryos display initial gas filling of the tracheal system which then refills with liquid shortly after hatching of the larvae (chapter 2.2.2). These observations implicate that the incomplete luminal aECM clearance and detached tracheal cuticle in tpr mutant embryos 
(chapter 2.2.5) still allows LC of the tracheal tubes. The role of Tpr in tracheal gas filling may therefore not be restricted to its functions in tracheal aECM maturation. Tpr might also affect tracheal gas filling by regulation of liquid homeostasis, which corresponds to the function of vertebrate prostasin and will be discussed in the following chapter.

\subsection{Conserved function of the matriptase-prostasin proteolytic cascade during epithelial morphogenesis}

Rescue experiments identified the human TTSP matriptase and human serine protease prostasin as functional homologs of $D$. melanogaster $\mathrm{Np}$ and $\mathrm{Tpr}$, respectively. Evidently, ectopic expression of human matriptase in the embryonic tracheal system of $N p$ mutant embryos rescues all aspects of the $N p$ mutant phenotype, i.e. detachment of the intraluminal aECM cable from apical membranes, degradation of luminal Dpy, taenidial folds formation, and gas filling of the tracheal system (chapter 2.1.16). Similarly, ectopic expression of human prostasin in the developing tracheal system of tpr mutant embryos facilitates wildtype-like tracheal gas filling and prevents refilling of tracheal tubes during larval development (chapter 2.2.6). However, secretion of human matriptase and prostasin into the tracheal lumen, which facilitates proper functioning during tracheal development, requires their human co-factor HAI-2 (chapters 2.1.16 and 2.2.6). These observations are in agreement with the role of HAI-2 in regulation of matriptase and prostasin translocation to cellular membranes and ectodomain shedding of matriptase (Nonboe et al. 2017; Friis et al. 2014). The requirement of human HAI-2 for matriptase and prostasin function in the $D$. melanogaster tracheal system indicates that the regulatory role of HAI-2 is not conserved across humans and flies. Regulation of activity and membrane-anchoring of $D$. melanogaster $\mathrm{Np}$ and $\mathrm{Tpr}$ therefore likely evolved differently as for matriptase and prostasin in mammals. These differences in regulatory mechanisms might also account for the different domain structures of matriptase compared to $\mathrm{Np}$, and prostasin compared to Tpr. The extracellular stem of human matriptase contains a SEA domain, two CUB domains and four LDLa domains which were shown to contribute to regulation of activity, subcellular localization, and ectodomain 
shedding of matriptase (Friis et al. 2014; Inouye et al. 2010; Lin et al. 2008). In contrast, the extracellular stem of $\mathrm{Np}$ contains no predicted protein domains but many predicted glycosylation sites (chapter 2.1.1; Steentoft et al. 2013). While Tpr and human prostasin both contain a canonical signal peptide for secretion, a prodomain and a catalytic domain, prostasin additionally contains a GPI-anchor site (chapter 2.2.1). Nevertheless, Tpr also shows membrane-associated localization in the tracheal system (chapter 2.2.3), indicating different mechanisms of membraneanchoring for prostasin and Tpr.

Tracheal co-expression of matriptase and HAI-2 rescues all aspects of the $N p$ mutant tracheal phenotype, indicating that function of human matriptase in the $D$. melanogaster tracheal system does not require its human co-factor HAI-1 (chapter 2.1.16). It is well-established that HAI-1 is the main inhibitor of matriptase proteolytic activity in mammals and also regulates autocatalytic activation of the matriptase zymogen (Mitchell et al. 2018; Friis et al. 2014; Lin et al. 2008). Accordingly, down-regulated HAI-1 expression in mammalian tissues is associated with developmental defects and tumor formation caused by deregulation of matriptase activity (Miller and List 2013). The observation that matriptase can substitute for $\mathrm{Np}$ functions in D. melanogaster tracheal development without coexpression of HAI-1 therefore suggests that some aspects of catalytic activity regulation of matriptase and $\mathrm{Np}$ are conserved across vertebrates and invertebrates. These putatively conserved mechanisms might particularly regulate autocatalytic zymogen activation since both, $\mathrm{Np}$ and matriptase show autocatalytic zymogen activation in vivo and in vitro (chapters 2.1.14 and 2.1.17; Szabo and Bugge 2011; Lee et al. 2007).

While regulatory mechanisms are not fully conserved, the obtained results indicate highly conserved functions and substrate specificities of the matriptase-prostasin proteolytic cascade. In mammals, matriptase is known to activate the prostasin zymogen by proteolytic cleavage (Netzel-Arnett et al. 2006). Correspondingly, Np and matriptase cleave Tpr at its zymogen activation site (chapter 2.2.6). This indicates conserved functional relationships between matriptase-prostasin in mammals and Np-Tpr in D. melanogaster. However, expression of Tpr in the $D$. melanogaster embryo is restricted to the tracheal system (chapters 2.2.1 and 2.2.3), while vertebrate prostasin is widely co-expressed with matriptase in ectodermal 
tissues (Miller and List 2013). Since Np is expressed in most ectodermal embryonic tissues (chapter 2.1.3), I propose that other proteases that are similar to Tpr

execute downstream functions of $\mathrm{Np}$ in non-tracheal ectodermal tissues. Tpr belongs to a group of seven highly similar D. melanogaster proteases (Ross et al. 2003). The corresponding genes are differentially expressed in specific spatial patterns in various embryonic ectodermal tissues (Gramates et al. 2017). These protease might therefore mediate Tpr-like functions in the different ectodermal tissues that express $\mathrm{Np}$, including the salivary glands, hindgut, and epidermis. In addition to cleavage of the Tpr zymogen, $\mathrm{Np}$ and matriptase proteolytically cleave the D. melanogaster ZPD protein Pio in vitro (chapter 2.1.17). Substitution of a single arginine with alanine in the ZPD of Pio provides cleavage resistance against both, $\mathrm{Np}$ and matriptase (chapter 2.1.17), indicating a highly conserved substrate specificity. Studies on the role of the Np-Tpr proteolytic cascade in D. melanogaster might therefore contribute to understanding of the mechanisms and proteolytic targets of the vertebrate matriptase-prostasin proteolytic cascade.

\subsubsection{Conserved role of the matriptase-prostasin proteolytic cascade in epithelial barrier function and liquid homeostasis regulation}

The matriptase-prostasin proteolytic cascade is known to regulate establishment of the epithelial barrier function in the mammalian epidermis and intestine (Miller and List 2013). While underlying mechanisms are not fully understood, it was shown that matriptase affects turn-over of claudins which are core-components of the barrier function providing tight junctions (Wu et al. 2017; Leyvraz et al. 2005). In the intestine, matriptase stabilizes the tight junction component claudin- 7 by cleavage of the tight junction scaffold protein EpCAM (Wu et al. 2017). Corresponding to the role of matriptase in vertebrates, $\mathrm{Np}$ regulates maintenance of the transepithelial barrier in the D. melanogaster tracheal system (chapter 2.1.5). However, $\mathrm{Np}$ does not affect formation and ultrastructural morphology of the septate junctions (chapters 2.1.4 and 2.1.5), which are the analogous structures of vertebrate tight junctions. The role in claudin turn-over regulation might nevertheless be conserved between matriptase and $\mathrm{Np}$ since claudins are also corecomponents of the invertebrate septate junctions (Furuse et al. 2006; Behr et al. 2003). Absence of detectable morphological defects in the septate junctions of $N p$ 
mutant trachea may be due to the relatively slow turn-over of septate junction components (Babatz et al. 2018). Thus, Np-mediated regulation of this relatively slow turn-over process might not affect the structure of septate junctions but may be essential for their function. However, further experiments are needed to verify the proposed role of $\mathrm{Np}$ in claudin turn-over.

In contrast to $\mathrm{Np}$, Tpr is not essential for the tracheal transepithelial barrier function (chapter 2.2.4). This indicates that the role of $\mathrm{Np}$ in epithelial barrier function is not dependent on activation of Tpr. Instead, $\mathrm{Np}$ might regulate epithelial barrier function via activation of Lint, since lint mutant embryos also lack tracheal transepithelial barrier function in stage 17 (Fig 54D). While Tpr is not implicated in epithelial barrier function regulation in D. melanogaster, its vertebrate functional homolog prostasin is essential for epithelial barrier function by regulating tight junction integrity (Miller and List 2013; Leyvraz et al. 2005). These observations suggest different setups in the downstream proteolytic cascades of $\mathrm{Np}$ and vertebrate matriptase, while mediating similar functions. Tpr and prostasin also might have similar function in regulation of ENaCs. It is wellestablished that prostasin is required for activation of ENaCs in the mammalian airways, epidermis and intestine and thereby regulates epithelial liquid homeostasis (Carattino et al. 2014; Miller and List 2013). The activity of ENaCs is also implicated in gas filling of the D. melanogaster tracheal system (Liu et al. 2003). Therefore, Tpr might mediate tracheal LC by activation of ENaCs, corresponding to the role of prostasin in vertebrates.

\subsubsection{Zona pellucida domain proteins as novel targets of the matriptase-prostasin proteolytic cascade}

During development of the tracheal system, $\mathrm{Np}$ and Tpr mediate degradation of the ZPD protein Dpy in the tracheal lumen and $\mathrm{Np}$ facilitates ectodomain shedding of the ZPD protein Pio by proteolytic cleavage (chapters 2.1.8, 2.1.10, 2.1.12 and 2.2.5). Human matriptase also mediates intraluminal Dpy degradation and cleaves Pio at the equivalent position as Np (chapters 2.1.16 and 2.1.17). Furthermore, the human ZPD protein TGFBR3 is cleaved by matriptase and $\mathrm{Np}$ within its extracellular domain (chapter 2.1.18). These observations indicate that ZPD 
proteins might represent conserved targets of the matriptase-prostasin proteolytic cascade.

In the $D$. melanogaster embryo, regulation of ZPD proteins by $\mathrm{Np}$ is not restricted to the tracheal system since $\mathrm{Np}$ is also essential for a uniform distribution of Dpy and Pio in the epidermis (chapter 2.1.11). In addition, $\mathrm{Np}$ mediates organization of the epidermal cuticle and formation of the cuticle denticle belts (chapter 2.1.7). While Dpy and Pio are implicated in attachment of the epidermis to the cuticle, eight other ZPD proteins were shown to play crucial roles epidermal cuticle development and denticle formation in particular (Fernandes et al. 2010; Bökel et al. 2005; Wilkin et al. 2000). Therefore, Np might also target these other ZPD proteins in the epidermal aECM, suggesting a general mechanism of ZPD-protein regulation by the functional matriptase homolog $\mathrm{Np}$. Since ZPD proteins are found in virtually all animals, their regulation by the matriptase-prostasin proteolytic cascade might also play an important role in vertebrate tissues (Jovine et al. 2005). ZPD proteins are known constituents of various aECM structures of vertebrate tissues and fulfill diverse functions. For instance, ZP1-ZP3 are essential in the mammalian ovary for fertilization, uromodulin controls salt transport and urine concentration by polymerizing into a gel-like matrix in the tubular kidney lumen and the alpha- and beta-tectorins are essential for sound transmission in the cochlear (Schaeffer et al. 2009; Jovine et al. 2005; Verhoeven et al. 1998). Furthermore, The mammalian TGF- $B$ receptor TGFBR3 contains a ZPD and plays central roles in cancer development and progression by promoting TGF- $B$ signaling (Zhang et al. 2016; Todorovic-Rakovic et al. 2011; Gatza et al. 2010). Shedding of the TGFBR3 ectodomain is furthermore known to down-regulate TGF- 8 signaling by extracellular binding of ligands (Elderbroom et al. 2014; Philip et al. 1999). The observed cleavage of TGFBR3 by Np and matriptase likely represents shedding of its ectodomain (chapter 2.1.18). Therefore, these results might indicate a novel mechanisms which contributes to the well-established role of matriptase in cancer formation and progression (Tanabe and List 2017). Indeed, cell culture-based experiments already suggest a functional role of matriptase-mediated TGFBR3 ectodomain shedding. Cultured D. melanogaster cells that express human TGFBR3 display increased filopodia formation, which is in accordance with a study that identified TGFBR3 as a promotor of filopodia formation in human mammary epithelial cells (Oh et al. 2013). Strikingly, co-expression of TGFBR3 with Np or 
human matriptase suppresses filopodia formation (chapter 2.1.18). This suggests that ectodomain shedding of TGFBR3 inhibits its ability to induce filopodia formation.

In summary, the results of this thesis indicate that D. melanogaster ZPD proteins and mammalian ZPD proteins are targeted by the Np-Tpr and matriptaseprostasin proteolytic cascades. Therefore I propose that ZPD protein cleavage by the matriptase-prostasin proteolytic cascade may represent a conserved process for regulation of ECM remodeling and signaling pathways in development and pathogenic conditions. 


\section{Materials and methods}

\subsection{Materials}

Table 1: List of suppliers

\begin{tabular}{ll}
\hline Supplier & Location \\
\hline abcam & Cambridge, UK \\
\hline Adobe Systems & Munich, Germany \\
\hline Becker's Bester & Lütgenrode, Germany \\
\hline $\begin{array}{l}\text { Berkeley Drosophila Genome Project } \\
\text { (BDGP) }\end{array}$ & Berkeley, USA \\
\hline BestGene & Chino Hills, USA \\
\hline Biometra & Göttingen, Germany \\
\hline BioRad & Hercules, USA \\
\hline Biozym & Hessisch Oldendorf, Germany \\
\hline $\begin{array}{l}\text { Bloomington Drosophila Stock Center } \\
\text { (BDSC) }\end{array}$ & Bloomington, USA \\
\hline Colgate-Palmolive & Hamburg, Germany \\
\hline Cornexo & Freimersheim, Germany \\
\hline $\begin{array}{l}\text { Department of Drosophila Genomics } \\
\text { and Genetic Resources (DGGR) }\end{array}$ & Kyoto, Japan \\
\hline $\begin{array}{l}\text { Developmental Studies Hybridoma } \\
\text { Bank (DSHB) }\end{array}$ & Iowa City, USA; \\
\hline Diagenode & Seraing, Belgium \\
\hline DNAStar & Madison, USA \\
\hline $\begin{array}{l}\text { Drosophila Genomics Resource Center } \\
\text { (DGRC) }\end{array}$ & Bloomington, USA \\
\hline DSM Bakery Ingredients & Dordrecht, Netherlands \\
\hline Engineering Office M. Wohlwend & Sennwald, Germany \\
\hline Eppendorf & Hamburg, Germany \\
\hline Fermentas & St- Leon-Rot, Germany \\
\hline Fujifilm & Düsseldorf, Germany \\
\hline GE Healthcare & Freiburg, Germany \\
\hline
\end{tabular}




\begin{tabular}{|c|c|}
\hline GeneTex & Hsinchu City, Taiwan \\
\hline Gilson International & Den Haag, the Netherlands \\
\hline Grafschafter Krautfabrik & Meckenheim, Germany \\
\hline Greiner Bio One & Nürtingen, Germany \\
\hline IBA & Göttingen, Germany \\
\hline Jackson ImmunoResearch & Cambridgeshire, UK \\
\hline Leica Microsystems & Wetzlar, Germany \\
\hline Macherey-Nagel & Düren, Germany \\
\hline MatTek & Ashland, USA \\
\hline MeisterMarken Ulmer Spatz & Bingen am Rhein, Germany \\
\hline Merck & Darmstadt, Germany \\
\hline Microsynth Seqlab & Göttingen, Germany \\
\hline Millipore & Darmstadt, Germany \\
\hline Molecular Probes & Eugene, USA \\
\hline MWG Eurofins Genomics & Ebersberg, Germany \\
\hline National Institute of Genetics (NIG) & Mishima, Japan \\
\hline New England Biolabs (NEB) & Frankfurt, Germany \\
\hline Nippon Genetics Europe & Düren, Germany \\
\hline OriGene & Rockville, USA \\
\hline Philips & Amsterdam, the Netherlands \\
\hline Qiagen & Hilden, Germany \\
\hline R\&D Systems & Minneapolis, USA \\
\hline Roche & Basel, Switzerland \\
\hline Rockland & Limerick, USA \\
\hline Roth & Karlsruhe, Germany \\
\hline Sarstedt & Nürmbrecht, Germany) \\
\hline Sartorius Stedim Biotech & Göttingen, Germany \\
\hline Schott & Mainz, Germany \\
\hline Sino Biological & Beijing, China \\
\hline Soja Austria & Vienna, Austria \\
\hline Synaptic Systems & Göttingen, Germany \\
\hline Thermo Fisher Scientific & Waltham, USA \\
\hline $\begin{array}{l}\text { Vienna Drosophila RNAi Center } \\
\text { (VDRC) }\end{array}$ & Vienna, Austria \\
\hline VWR & Leuven, Belgium \\
\hline Zeiss & Oberkochen, Germany \\
\hline
\end{tabular}




\subsubsection{Buffers, reagents, solutions and nutrient media}

If not indicated otherwise, chemicals were obtained from Merck, Roche, Roth or VWR. Water for sterile buffers and solutions was run through a Milli-Q-System (Millipore). Sterile nutrient media and water were autoclaved at $121^{\circ} \mathrm{C}$ for 45 minutes. Liquids were stored in bottles (Schott), tubes (Sarstedt) or cups (Eppendorf). Buffers, reagents, solutions and nutrient media were stored at indicated temperatures or at room temperature (RT).

Table 2:List of buffers, reagents and solutions

\begin{tabular}{|c|c|}
\hline Identifier (storage temperature) & Composition \\
\hline BBS $10 x\left(-20^{\circ} \mathrm{C}\right)$ & $\begin{array}{l}100 \mathrm{mM} \text { Tris, } 550 \mathrm{mM} \mathrm{NaCl}, 400 \mathrm{mM} \mathrm{KCl} \text {, } \\
70 \mathrm{mM} \mathrm{MgCl} 2,50 \mathrm{mM} \mathrm{CaCl} 2,200 \mathrm{mM} \\
\text { Glucose, } 500 \mathrm{mM} \text { Sucrose, } \mathrm{pH} 7,0\end{array}$ \\
\hline $\mathrm{BBT}\left(4^{\circ} \mathrm{C}\right)$ & $\begin{array}{l}\text { BBS (1x), 0,1 \% (w/v) BSA, 0,1\%(v/v) } \\
\text { Tween-20 }\end{array}$ \\
\hline Bleach solution (RT) & $50 \%$ (v/v) DanKlorix (Colgate-Palmolive) \\
\hline Blocking solution $\left(4{ }^{\circ} \mathrm{C}\right)$ & BBT (1x), $2 \%$ (v/v) Goat-Serum \\
\hline Ethidium-bromide solution $\left(4^{\circ} \mathrm{C}\right)$ & $10 \mathrm{mg} / \mathrm{ml}$ \\
\hline Formaldehyde solution $\left(4^{\circ} \mathrm{C}\right)$ & $5 \%(\mathrm{v} / \mathrm{v})$ Formaldehyde in PBS $(1 \mathrm{x})$ \\
\hline Hepes solution $\left(4^{\circ} \mathrm{C}\right)$ & $\begin{array}{l}100 \mathrm{mM} \text { Hepes, } 2 \text { mM MgSO4, pH 6,8, } 1 \\
\text { mM EDTA }\end{array}$ \\
\hline Hoyer's medium $\left(4^{\circ} \mathrm{C}\right)$ & $\begin{array}{l}90 \mathrm{~g} \text { gum arabic, } 400 \mathrm{~g} \text { chloral hydrate, } 40 \mathrm{~g} \\
\text { glycerol per } 100 \mathrm{ml} \mathrm{H} 2 \mathrm{O} \text {, centrifuged and } \\
\text { filtered through glass wool. }\end{array}$ \\
\hline Hybridization-Solution $\left(-20^{\circ} \mathrm{C}\right)$ & $\begin{array}{l}50 \%(\mathrm{v} / \mathrm{v}) \text { Formamide (deionized); } 5 x \text { SSC; } \\
200 \mu \mathrm{g} / \mathrm{ml} \text { ss DNA; } 10 \mu \mathrm{g} / \mathrm{ml} \text { tRNA; } 50 \\
\mu \mathrm{g} / \mathrm{ml} \text { Heparin; pH } 5.0\end{array}$ \\
\hline Lysis buffer $\left(4^{\circ} \mathrm{C}\right)$ & $\begin{array}{l}10 \mathrm{mM} \text { Tris/HCl, } 150 \mathrm{mM} \mathrm{NaCl}, 0.5 \% \mathrm{NP}- \\
40, \mathrm{pH} 7.5\end{array}$ \\
\hline Nipagin solution $(\mathrm{RT})$ & $15 \%$ (v/v) Nipagin in Ethanol (95 \%) \\
\hline PBS 10x (RT) & $\begin{array}{l}1,3 \mathrm{M} \mathrm{NaCl}, 100 \mathrm{mM} \text { Na2HPO4-Buffer, } \mathrm{pH} \\
7,4\end{array}$ \\
\hline $\mathrm{PBT}(\mathrm{RT})$ & PBS (1x), 0,1 \% (v/v) Tween-20 \\
\hline PBTX (RT) & PBS (1x); $0.5 \%$ (v/v) Triton-X-100 \\
\hline Pre-hybridization-solution $\left(-20^{\circ} \mathrm{C}\right)$ & $50 \%$ (v/v) Formamide (deionized); 5x SSC \\
\hline Protease reaction buffer $(\mathrm{RT})$ & $\begin{array}{l}100 \mathrm{mM} \text { Tris/HCl, } 50 \mathrm{mM} \mathrm{NaCl}, 2 \mathrm{mM} \\
\mathrm{CaCl}, \mathrm{pH} 8.5\end{array}$ \\
\hline Proteinase-K solution $\left(-20^{\circ} \mathrm{C}\right)$ & $5 \mathrm{mg}$ Proteinase-K/ml \\
\hline
\end{tabular}




\begin{tabular}{|c|c|}
\hline RNA-fix solution $\left(-20^{\circ} \mathrm{C}\right)$ & $\begin{array}{l}10 \% \text { (v/v) Formaldehyde solution, } 50 \mathrm{mM} \\
\text { EGTA, pH7,0 }\end{array}$ \\
\hline SDS-running buffer (RT) & $\begin{array}{l}100 \mathrm{mM} \text { Tris, } 100 \mathrm{mM} \text { Hepes, } 3 \mathrm{mM} \text { SDS, } \\
\text { pH } 8,0\end{array}$ \\
\hline SDS-sample buffer $4 \mathrm{x}\left(-20^{\circ} \mathrm{C}\right)$ & $\begin{array}{l}\text { 0,5 M Tris-HCl, } 20 \%(\mathrm{w} / \mathrm{v}) \text { SDS, } 40 \%(\mathrm{v} / \mathrm{v}) \\
\text { Glycerol, 0,004 \% (w/v) Bromophenol Blue, } \\
10 \%(\mathrm{v} / \mathrm{v}) \text { B-Mercaptoethanol, pH 6,8 }\end{array}$ \\
\hline Squishing buffer (RT) & $\begin{array}{l}10 \mathrm{mM} \text { Tris, } 25 \mathrm{mM} \mathrm{NaCl}, 1 \mathrm{mM} \text { EDTA, } \mathrm{pH} \\
8,2\end{array}$ \\
\hline TAE 50x (RT) & $\begin{array}{l}2 \text { M Tris, } 1 \text { M Acetic acid, } 50 \text { mM EDTA, } \\
\text { pH } 8,4\end{array}$ \\
\hline TCA solution $\left(4{ }^{\circ} \mathrm{C}\right)$ & $\begin{array}{l}500 \mathrm{~g} \text { Trichloroacetic acid (TCA) in } 350 \mathrm{ml} \\
\text { water }\end{array}$ \\
\hline Transfer buffer (RT) & $\begin{array}{l}25 \mathrm{mM} \text { Tris, } 190 \mathrm{mM} \text { Glycine, } 20 \% \text { (v/v) } \\
\text { methanol }\end{array}$ \\
\hline AP-buffer (RT) & $\begin{array}{l}100 \mathrm{mM} \text { Tris/HCL, } 100 \mathrm{mM} \mathrm{NaCl}, 50 \mathrm{mM} \\
\mathrm{MgCl} 2,1 \mathrm{mM} \text { Levamisole, } 0,1 \%(\mathrm{v} / \mathrm{v}) \text { Tween } \\
20, \mathrm{pH} 9,5\end{array}$ \\
\hline
\end{tabular}

Table 3: List of nutrient media

\begin{tabular}{|c|c|}
\hline Identifier (storage temperature) & Composition or supplier \\
\hline Apple juice agar plates $\left(4^{\circ} \mathrm{C}\right)$ & $\begin{array}{l}33 \%(\mathrm{v} / \mathrm{v}) \text { apple juice (Becker's Bester) } \\
3,3 \%(\mathrm{w} / \mathrm{v}) \text { sucrose, } 2,8 \%(\mathrm{w} / \mathrm{v}) \text { agarose, } \\
1,3 \%(\mathrm{v} / \mathrm{v}) \text { Nipagin-solution }\end{array}$ \\
\hline Fly food & $\begin{array}{l}0,63 \%(\mathrm{w} / \mathrm{v}) \text { Agarose, } 1,8 \%(\mathrm{w} / \mathrm{v}) \\
\text { inactive dry Yeast (DMS Bakery } \\
\text { Ingredients), } 1 \%(\mathrm{w} / \mathrm{v}) \text { soy bean powder } \\
\text { (Soja Austria), } 8 \%(\mathrm{w} / \mathrm{v}) \text { cornmeal } \\
\text { Cornexo), } 2,2 \%(\mathrm{v} / \mathrm{v}) \text { light corn syrup } \\
\text { (Grafschafter Krautfabrik), } 8 \%(\mathrm{v} / \mathrm{v}) \\
\text { malt extract (MeisterMarken Ulmer } \\
\text { Spatz), 0,63\% (v/v) propionic-acid, 0,15 } \\
\%(\mathrm{v} / \mathrm{v}) \text { Nipagin-solution, in } \mathrm{dH} 2 \mathrm{O}\end{array}$ \\
\hline LB (lysogeny broth)-medium & $\begin{array}{l}0,5 \%(\mathrm{w} / \mathrm{v}) \mathrm{NaCl}, 0,5 \%(\mathrm{w} / \mathrm{v}) \text { Yeast- } \\
\text { extract, } 0,1 \%(\mathrm{w} / \mathrm{v}) \text { Glucose, } \mathrm{pH} 7,0\end{array}$ \\
\hline LB plates with Ampicillin & $\begin{array}{l}15 \mathrm{~g} / \mathrm{L} \text { Agar in LB-medium with } \\
\text { Ampicilling }\end{array}$ \\
\hline LB plates with Kanamycin & $\begin{array}{l}15 \text { g/L Agar in LB-medium with } \\
\text { Kanamycin }\end{array}$ \\
\hline LB-medium with Ampicillin & $100 \mu \mathrm{g} / \mathrm{ml}$ Ampicillin in LB-medium \\
\hline LB-medium with Kanamycin & $50 \mu \mathrm{g} / \mathrm{ml}$ Kanamycin in LB-medium \\
\hline Schneiders Drosophila medium $\left(4^{\circ} \mathrm{C}\right)$ & Thermo Fisher Scientific \\
\hline
\end{tabular}




\subsubsection{Commercially available kits, enzymes and reagents}

All restriction endonucleases were ordered from NEB. Kits, enzymes and reagents were stored according to suppliers' guidelines.

Table 4: List of commercially available kits, enzymes and reagents

\begin{tabular}{|c|c|}
\hline Identifier & Supplier \\
\hline Casein Blocking Buffer 10x & Sigma-Aldrich \\
\hline Chitin-Binding-Probe (CBP) FITC-conjugated & NEB \\
\hline Chitin-Binding-Probe (CBP) Rhodamin-conjugated & NEB \\
\hline CloneJet PCR Cloning Kit & Fermentas \\
\hline DIG RNA Labeling Kit & Sigma-Aldrich \\
\hline DNA loading dye $6 \mathrm{X}$ & Thermo Fisher Scientific \\
\hline DNeasy Blood \& Tissue Kit & Qiagen \\
\hline dNTP Mix $10 \mathrm{mM}$ each & Thermo Fisher Scientific \\
\hline Effectene Transfection Reagent & Qiagen \\
\hline FITC-conjugated 70 kDa Dextran & Molecular Probes \\
\hline GeneRuler 1 kb DNA ladder ready-to-use & Thermo Fisher Scientific \\
\hline HotStarTaq Master Mix Kit & Qiagen \\
\hline HyClone Fetal Bovine Serum (FBS) & GE Healthcare \\
\hline Midori Green & Nippon Genetics Europe \\
\hline NBT/BCIP Substrate Solution & Thermo Fisher Scientific \\
\hline NEBuilder HiFi DNA Assembly Master Mix & NEB \\
\hline NucleoSpin Gel and PCR Clean-up Kit & Macherey-Nagel \\
\hline NucleoSpin Plasmid Kit & Macherey-Nagel \\
\hline PageRuler prestained protein ladder & Thermo Fisher Scientific \\
\hline Penicillin-Streptomycin & Thermo Fisher Scientific \\
\hline Phusion-HF DNA polymerase & NEB \\
\hline Plasmid Plus Midi Kit & Qiagen \\
\hline ProLong Diamond Antifade Mountant & Thermo Fisher Scientific \\
\hline ProLong Gold Antifade Mountant & Thermo Fisher Scientific \\
\hline Q5-HF DNA polymerase & NEB \\
\hline Quick Ligation kit & NEB \\
\hline Superscript IV Kit & Thermo Fisher Scientific \\
\hline T4 DNA Ligase & NEB \\
\hline Texas Red-conjugated 10 kDa Dextran & Molecular Probes \\
\hline WesternBright Sirius Kit & Biozym \\
\hline
\end{tabular}




\subsubsection{Primary and secondary antibodies}

Antibodies were stored for long-term at $-80{ }^{\circ} \mathrm{C}$ and at $4{ }^{\circ} \mathrm{C}$ after thawing.

Table 5: List of antibodies

\begin{tabular}{|c|c|c|c|c|}
\hline Antibody & Host & Dilution* & Source & Identifier \\
\hline \multicolumn{5}{|c|}{ Primary antibodies } \\
\hline anti-Crb & mouse & IF: $1: 50$ & DSHB & $\mathrm{Cq} 4$ \\
\hline anti-DE-cad & rat & IF: $1: 50$ & DSHB & DECAD2 \\
\hline anti-Fas3 & mouse & IF: $1: 500$ & DSHB & $7 \mathrm{G} 10$ \\
\hline anti-Flag & mouse & WB: 1:10000 & Merck & F3165 \\
\hline anti-GFP & chicken & IF: $1: 1000$ & Abcam & ab13970 \\
\hline anti-GFP & rabbit & $\begin{array}{l}\text { IF: } 1: 500 \\
\text { WB: } 1: 10000 \\
\text { CIF: } 1: 1000\end{array}$ & Synaptic Systems & 132003 \\
\hline anti-HAI-2 & rabbit & IF: $1: 200$ & $\begin{array}{l}\text { Thermo Fisher } \\
\text { Scientific }\end{array}$ & PA5-52790 \\
\hline anti-Kune & rabbit & IF: $1: 500$ & Nelson et al. 2010 & - \\
\hline anti-matriptase & sheep & $\begin{array}{l}\text { IF: } 1: 200 \\
\text { WB: } 1: 10000\end{array}$ & R\&D Systems & AF3946 \\
\hline anti-mCherry & rabbit & $\begin{array}{l}\text { IF: } 1: 500 \\
\text { WB: } 1: 10000\end{array}$ & Rockland & 600-401-P16 \\
\hline anti-Mega & mouse & IF: $1: 50$ & Behr et al. 2003 & - \\
\hline anti-prostasin & rabbit & IF: $1: 200$ & GeneTex & GTX103976 \\
\hline anti-Shot & mouse & IF: $1: 250$ & DSHB & mAbRod 1 \\
\hline anti-Spectrin & mouse & IF: $1: 10$ & DSHB & $3 \mathrm{~A} 9$ \\
\hline anti-Strep & mouse & CIF: 1:1000 & IBA & 2-1507-001 \\
\hline anti-Strep-HRP & mouse & WB: 1:10000 & IBA & 2-1509-001 \\
\hline anti-Strep & rabbit & $\begin{array}{l}\text { IF: } 1: 250 \\
\text { WB: } 1: 1000\end{array}$ & abcam & ab76949 \\
\hline anti-Uif & Guinea pig & IF: $1: 500$ & $\begin{array}{l}\text { Zhang and Ward } \\
2009\end{array}$ & - \\
\hline anti-B-tubulin & mouse & WB 1:100 & DSHB & E7 \\
\hline \multicolumn{5}{|c|}{ Secondary antibodies } \\
\hline $\begin{array}{l}\text { anti-chicken- } \\
\text { AlexaFluor488 }\end{array}$ & goat & IF: $1: 500$ & $\begin{array}{l}\text { Thermo Fisher } \\
\text { Scientific }\end{array}$ & A-11039 \\
\hline $\begin{array}{l}\text { anti-chicken- } \\
\text { DyLight594 }\end{array}$ & donkey & IF: $1: 500$ & $\begin{array}{l}\text { Jackson Immuno } \\
\text { Research }\end{array}$ & $703-516-155$ \\
\hline anti-DIG-AP & sheep & IF: $1: 2000$ & Roche & 11093274910 \\
\hline
\end{tabular}




\begin{tabular}{|c|c|c|c|c|}
\hline $\begin{array}{l}\text { anti-guinea pig- } \\
\text { AlexaFluor488 }\end{array}$ & goat & IF: $1: 500$ & $\begin{array}{l}\text { Thermo Fisher } \\
\text { Scientific }\end{array}$ & A-11073 \\
\hline $\begin{array}{l}\text { anti-guinea pig- } \\
\text { AlexaFluor568 }\end{array}$ & goat & IF: $1: 500$ & $\begin{array}{l}\text { Thermo Fisher } \\
\text { Scientific }\end{array}$ & A-11075 \\
\hline $\begin{array}{l}\text { anti-mouse- } \\
\text { AlexaFluor } 405\end{array}$ & goat & IF: $1: 500$ & $\begin{array}{l}\text { Thermo Fisher } \\
\text { Scientific }\end{array}$ & A-31553 \\
\hline $\begin{array}{l}\text { anti-mouse- } \\
\text { AlexaFluor488 }\end{array}$ & goat & IF: $1: 500$ & $\begin{array}{l}\text { Thermo Fisher } \\
\text { Scientific }\end{array}$ & A-21121 \\
\hline $\begin{array}{l}\text { anti-mouse- } \\
\text { AlexaFluor568 }\end{array}$ & goat & IF: $1: 500$ & $\begin{array}{l}\text { Thermo Fisher } \\
\text { Scientific }\end{array}$ & A-21124 \\
\hline $\begin{array}{l}\text { anti-mouse- } \\
\text { AlexaFluor647 }\end{array}$ & goat & IF: $1: 500$ & $\begin{array}{l}\text { Thermo Fisher } \\
\text { Scientific }\end{array}$ & A-21235 \\
\hline anti-mouse-HRP & goat & WB: 1:10000 & $\begin{array}{l}\text { Thermo Fisher } \\
\text { Scientific }\end{array}$ & G-21040 \\
\hline $\begin{array}{l}\text { anti-rabbit- } \\
\text { AlexaFluor488 }\end{array}$ & goat & IF: $1: 500$ & $\begin{array}{l}\text { Thermo Fisher } \\
\text { Scientific }\end{array}$ & A-11034 \\
\hline $\begin{array}{l}\text { anti-rabbit- } \\
\text { AlexaFluor568 }\end{array}$ & goat & IF: $1: 500$ & $\begin{array}{l}\text { Thermo Fisher } \\
\text { Scientific }\end{array}$ & A-21069 \\
\hline anti-rabbit-HRP & goat & WB: 1:10000 & $\begin{array}{l}\text { Thermo Fisher } \\
\text { Scientific }\end{array}$ & G-21234 \\
\hline $\begin{array}{l}\text { anti-sheep- } \\
\text { AlexaFluor568 }\end{array}$ & donkey & IF: $1: 500$ & $\begin{array}{l}\text { Thermo Fisher } \\
\text { Scientific }\end{array}$ & A-21099 \\
\hline anti-sheep-HRP & donkey & WB: 1:10000 & $\begin{array}{l}\text { Thermo Fisher } \\
\text { Scientific }\end{array}$ & A16047 \\
\hline
\end{tabular}

\subsubsection{Oligonucleotides, plasmids and synthesized dsDNA fragments}

Oligonucleotides were synthesized in High Purity Salt Free (HPSF) quality by MWG Eurofins Genomics. Stock solutions $(100 \mu \mathrm{M})$ of oligonucleotides and plasmids were stored at $-20{ }^{\circ} \mathrm{C}$. Fragments of dsDNA were synthesized by MWG Eurofins Genomics and are listed in Appendix 7.1.

Table 6: List of oligonucleotides

\begin{tabular}{|c|c|c|}
\hline Identifier & Sequence & Purpose \\
\hline Np-sgRNA1-sense & $\begin{array}{l}\text { CTTCGGTGAGTGGCATTCT } \\
\text { AGAAG }\end{array}$ & \multirow[t]{2}{*}{$\begin{array}{l}\text { Annealed and cloned into } \\
p B F_{V}-U 6.2 \text { via BbsI }\end{array}$} \\
\hline Np-sgRNA1-antisense & $\begin{array}{l}\text { AAACCTTCTAGAATGCCAC } \\
\text { TCACC }\end{array}$ & \\
\hline
\end{tabular}




\begin{tabular}{|c|c|c|}
\hline Np-HDR-sgRNA2-sense & $\begin{array}{l}\text { CTTCGACAAGCGTTTCCAC } \\
\text { CTCGG }\end{array}$ & \multirow[t]{2}{*}{$\begin{array}{l}\text { Annealed and cloned into } \\
p B F_{V}-U 6.2 \text { via BbsI }\end{array}$} \\
\hline $\begin{array}{l}\text { Np-HDR-sgRNA2- } \\
\text { antisense }\end{array}$ & $\begin{array}{l}\text { AAACCCGAGGTGGAAACG } \\
\text { CTTGTC }\end{array}$ & \\
\hline tpr-sgRNA1-sense & $\begin{array}{l}\text { CTTCGTTGTGTCTGCTGAT } \\
\text { CTGCC }\end{array}$ & \multirow[t]{2}{*}{$\begin{array}{l}\text { Annealed and cloned into } \\
p B F_{V}-U 6.2 \text { via BbsI }\end{array}$} \\
\hline tpr-sgRNA1-antisense & $\begin{array}{l}\text { AAACGGCAGATCAGCAGA } \\
\text { CACAAC }\end{array}$ & \\
\hline tpr-HDR-sgRNA2-sense & $\begin{array}{l}\text { CTTCGCCCCAACGTCCCGG } \\
\text { CAGTT }\end{array}$ & \multirow{2}{*}{$\begin{array}{l}\text { Annealed and cloned into } \\
p B F_{V}-U 6.2 B \text { via BbsI }\end{array}$} \\
\hline $\begin{array}{l}\text { tpr-HDR-sgRNA2- } \\
\text { antisense }\end{array}$ & $\begin{array}{l}\text { AAACAACTGCCGGGACGT } \\
\text { TGGGGC }\end{array}$ & \\
\hline lint-sgRNA-sense & $\begin{array}{l}\text { CTTCGTGGGTTACGCCGG } \\
\text { CCTCAA }\end{array}$ & \multirow[t]{2}{*}{$\begin{array}{l}\text { Annealed and cloned into } \\
p B F_{V}-U 6.2 \text { via BbsI }\end{array}$} \\
\hline lint-sgRNA-antisense & $\begin{array}{l}\text { AAACTTGAGGCCGGCGTA } \\
\text { ACCCAC }\end{array}$ & \\
\hline pio-sgRNA-sense & $\begin{array}{l}\text { CTTCGATTGGGACACCGA } \\
\text { GCCACT }\end{array}$ & \multirow[t]{2}{*}{$\begin{array}{l}\text { Annealed and cloned into } \\
p B F_{V}-U 6.2 \text { via BbsI }\end{array}$} \\
\hline pio-sgRNA-antisense & $\begin{array}{l}\text { AAACAGTGGCTCGGTGTC } \\
\text { CCAATC }\end{array}$ & \\
\hline Np-5'-arm-for & $\begin{array}{l}\text { GAATTCCGCCGTGGCCGC } \\
\text { GATTTG }\end{array}$ & \multirow{2}{*}{$\begin{array}{l}\text { Cloning of } N p 5^{\prime} \text { homology } \\
\text { arm into } p G X \text {-attP via EcoRI } \\
/ \text { KpnI }\end{array}$} \\
\hline Np-5'-arm-rev & $\begin{array}{l}\text { GGTACCCCTGGCACTCAGT } \\
\text { CATTTGGGC }\end{array}$ & \\
\hline Np-3'-arm-for & $\begin{array}{l}\text { ACTAGTGGTTTGATACTAA } \\
\text { ATG }\end{array}$ & \multirow{2}{*}{$\begin{array}{l}\text { Cloning of } N p 3^{\prime} \text { homology } \\
\text { arm into } p G X \text {-attP via SpeI / } \\
\text { XhoI }\end{array}$} \\
\hline Np-3'-arm-rev & $\begin{array}{l}\text { CTCGAGGCCCATTTCTCTA } \\
\text { ATCGCGAGCGGTCTGACT } \\
\text { CG }\end{array}$ & \\
\hline tpr-5'-arm-for & $\begin{array}{l}\text { GCGGCCGCCCAGGTCTTT } \\
\text { GGTGGAGG }\end{array}$ & \multirow{2}{*}{$\begin{array}{l}\text { Cloning of tpr } 5 \text { ' homology } \\
\text {-arm into } p H D \text { - } D \text { sRed-attP } \\
\text { via NotI / NdeI }\end{array}$} \\
\hline tpr-5'-arm-rev & $\begin{array}{l}\text { GGGCATATGACCCTTTTAG } \\
\text { CTTCCCAAGGAGAAGAG }\end{array}$ & \\
\hline tpr-3'-arm-for & $\begin{array}{l}\text { CCCACTAGTCGGTTTCCTT } \\
\text { TGGTGATTCTGG }\end{array}$ & \multirow{2}{*}{$\begin{array}{l}\text { Cloning of tpr } 3 \text { ' homology } \\
\text { arm into } p H D \text {-DsRed-attP } \\
\text { via SpeI / XhoI }\end{array}$} \\
\hline tpr-3'-arm-rev & $\begin{array}{l}\text { AAACTCGAGAGGAGCAGG } \\
\text { CATCCCT }\end{array}$ & \\
\hline pio-5'-arm1-for & $\begin{array}{l}\text { ACTAGTCCGAATTCGCAGG } \\
\text { TGATTATCGCCTCTCGGCC } \\
\text { ATCAG } \\
\end{array}$ & \multirow{4}{*}{$\begin{array}{l}\text { Assembly of pio } 5 \text { ' homology } \\
\text { arm with mCherry encoding } \\
\text { - sequence in pJET1.2 via SpeI } \\
\text { / HindIII and subsequent } \\
\text { cloning into pHD- } \\
\text { ScarlessDsRed via AarI }\end{array}$} \\
\hline pio-5'-arm1-rev & $\begin{array}{l}\text { AAGCTTCTTTAATTAAAGG } \\
\text { GGAAATTTCG }\end{array}$ & \\
\hline pio-5'-arm2-for & $\begin{array}{l}\text { ACTAGTGGCAAGCTTACTG } \\
\text { GCGATGGATTAGGCC }\end{array}$ & \\
\hline pio-5'-arm2-rev & $\begin{array}{l}\text { CACCTGCGATCTTAATCTT } \\
\text { GCCAGCGTCTGTC }\end{array}$ & \\
\hline pio-3'-arm-for & $\begin{array}{l}\text { TTAAGGAAGAGCACACAG } \\
\text { TTGGGCGCTTTGTTAGTCG }\end{array}$ & \multirow{2}{*}{$\begin{array}{l}\text { Cloning of pio } 3^{\prime} \text { homology } \\
\text { arm into } p H D \text { - } \\
\text { ScarlessDsRed via SapI }\end{array}$} \\
\hline pio-3'-arm-rev & $\begin{array}{l}\text { CGGGGAAGAGCGACGAGA } \\
\text { TTGCGCCGGAAAATAAG }\end{array}$ & \\
\hline
\end{tabular}




\begin{tabular}{|c|c|c|}
\hline Np-rescue-for & $\begin{array}{l}\text { GCGGCCGCAGTGATTAAA } \\
\text { ATGTTTAAGGCCCTGGC }\end{array}$ & \multirow{2}{*}{$\begin{array}{l}\text { Cloning of } N p \text { genomic region } \\
\text { into } p G E \text {-att } B \text { via NotI/KpnI } \\
\text { for rescue of } N p^{\text {attP }} \text { allele }\end{array}$} \\
\hline Np-rescue-rev & $\begin{array}{l}\text { GGTACCCAGTTAGTAAAAC } \\
\text { GCACGACAAC }\end{array}$ & \\
\hline Np-rescueGFP-for & $\begin{array}{l}\text { GAATTCCGGGACTGGATC } \\
\text { AACCAGATCCTGCAGTTCG } \\
\text { CTAGCGGCGGTATGGTGA } \\
\text { GCAAGGGCGAGGAGC }\end{array}$ & \multirow{2}{*}{$\begin{array}{l}\text { Insertion of eGFP encoding } \\
\text { sequence into } N p \text { genomic } \\
\text { region in } p G E \text {-attB-Np- } \\
\text { rescue via EcoRI/BmgBI and } \\
\text { adding a linker with NheI } \\
\text { restriction site between } N p \\
\text { and eGFP encoding sequence }\end{array}$} \\
\hline Np-rescueGFP-rev & $\begin{array}{l}\text { CACGTCCTAGGCATTCACT } \\
\text { AAGAGTCGGGCCGGGGCG } \\
\text { GGACCTACGGATCCTTGTA } \\
\text { CAGCTCGTCCATG }\end{array}$ & \\
\hline tpr-rescue-for & $\begin{array}{l}\text { GCGGCCGCAATTAATGTTG } \\
\text { GAACTCAACTCAACAGGG }\end{array}$ & \multirow{2}{*}{$\begin{array}{l}\text { Cloning of tpr genomic region } \\
\text { into } p G E \text {-att } B \text { via NotI / } \\
\text { KpnI for rescue of } t p r^{a t t P} \\
\text { allele }\end{array}$} \\
\hline tpr-rescue-rev & $\begin{array}{l}\text { GGTACCGCTCCCGAGAAA } \\
\text { GGTTAGCGC }\end{array}$ & \\
\hline UAS-tpr-ORF-for & $\begin{array}{l}\text { GAATTCATGAATCGAGCCT } \\
\text { GGTTG }\end{array}$ & \multirow{2}{*}{$\begin{array}{l}\text { Cloning of tpr ORF into } \\
p U A S T \text { via EcoRI / XbaI }\end{array}$} \\
\hline UAS-tpr-ORF-rev & $\begin{array}{l}\text { TCTAGACTACTTGATCTTC } \\
\text { TTGGTCTCC }\end{array}$ & \\
\hline UAS-tpr::Flag-rev & $\begin{array}{l}\text { TCTAGACTACTTATCATCA } \\
\text { TCATCCTTGTAATCGATAT } \\
\text { CGTGATCCTTGTAATCGCC } \\
\text { ATCGTGATCCTTGTAATCG } \\
\text { CCCTTGATCTTCTTGGTCT } \\
\text { CCTG }\end{array}$ & $\begin{array}{l}\text { Cloning of tpr::Flag into } \\
p U A S T \text { via EcoRI / XbaI } \\
\text { (PCRs with UAS-tpr-ORF- } \\
\text { for) }\end{array}$ \\
\hline UAS-tpr-ZL-for & $\begin{array}{l}\text { ACAGGCGGCCATAGTCGG } \\
\text { TGGTCAGGAAAC }\end{array}$ & \multirow{2}{*}{$\begin{array}{l}\text { In vitro mutagenesis of } \\
\text { tpr::Flag to generate } \\
t p r^{Z L}:: \text { Flag that was cloned } \\
\text { into } p U A S T \text { (PCRs with } \\
\text { UAS-tpr-ORF-for and UAS- } \\
\text { tpr::Flag-rev) }\end{array}$} \\
\hline UAS-tpr-ZL-rev & $\begin{array}{l}\text { CACCGACTATGGCCGCCT } \\
\text { GTATATTGGC }\end{array}$ & \\
\hline UAS-Np-ORF-for & $\begin{array}{l}\text { GCGGCCGCATGGGTGCTC } \\
\text { AACACACGGACAC }\end{array}$ & \multirow[t]{2}{*}{$\begin{array}{l}\text { Cloning of } N p \text { ORF into } \\
-p U A S T \text { via NotI / XhoI }\end{array}$} \\
\hline UAS-Np-ORF-rev & $\begin{array}{l}\text { CTCGAGCTAGAACTGCAG } \\
\text { GATCTGGTTGATC }\end{array}$ & \\
\hline UAS-Np::GFP-for & $\begin{array}{l}\text { GCTAGCGGCGGTATGGTG } \\
\text { AGCAAG }\end{array}$ & \multirow{2}{*}{$\begin{array}{l}\text { Cloning of } N p:: \text { GFP into } \\
\text { pUAST-NheI-linker via NheI } \\
\text { / XhoI }\end{array}$} \\
\hline UAS-Np::GFP-rev & $\begin{array}{l}\text { CTCGAGCTACGGATCCTTG } \\
\text { TAC }\end{array}$ & \\
\hline UAS-lint-ORF-for & $\begin{array}{l}\text { GAATTCATGTTTAAGTGGG } \\
\text { TTACGCCGGCC }\end{array}$ & \multirow{2}{*}{$\begin{array}{l}\text { Cloning of lint ORF into } \\
\text { - } U A A S T \text { via EcoRI / XbaI }\end{array}$} \\
\hline UAS-lint-ORF-rev & $\begin{array}{l}\text { TCTAGACTATTTCACGCCC } \\
\text { GTTATGCTGCG }\end{array}$ & \\
\hline UAS-matriptase-ORF-for & $\begin{array}{l}\text { GAATTCATGGGGAGCGAT } \\
\text { CGGGCCCGCAAGGG }\end{array}$ & \multirow{2}{*}{$\begin{array}{l}\text { Cloning of human matriptase } \\
\text { ORF into } p U A S T \text { via EcoRI / } \\
\text { XbaI }\end{array}$} \\
\hline UAS-matriptase-ORF-rev & $\begin{array}{l}\text { TCTAGACTATACCCCAGTG } \\
\text { TTCTCTTTG }\end{array}$ & \\
\hline
\end{tabular}




\begin{tabular}{|c|c|c|}
\hline $\begin{array}{l}\text { UAS-matriptase::Strep- } \\
\text { for }\end{array}$ & $\begin{array}{l}\text { ACTCTGAATAGGGAATTGG } \\
\text { GATGGGGAGCGATCGGGC } \\
\text { C }\end{array}$ & \multirow{2}{*}{$\begin{array}{l}\text { Cloning of matriptase::Strep } \\
\text { into } p U A S T \text { via Gibson } \\
\text { - assembly (PCRs with UAS- } \\
\text { for and UAS-rev) }\end{array}$} \\
\hline $\begin{array}{l}\text { UAS-matriptase::Strep- } \\
\text { rev }\end{array}$ & $\begin{array}{l}\text { GACTCCATGCACTACCGCC } \\
\text { GCTAGCTACCCCAGTGTTC } \\
\text { TCTTTGATCCAGTC }\end{array}$ & \\
\hline UAS-prostasin-for & $\begin{array}{l}\text { GAATTCATGGCCCAGAAG } \\
\text { GGGGTCCTG }\end{array}$ & \multirow{2}{*}{$\begin{array}{l}\text { Cloning of human prostasin } \\
\text { ORF into } p U A S T \text { via EcoRI / } \\
\text { XbaI }\end{array}$} \\
\hline UAS-prostasin-rev & $\begin{array}{l}\text { TCTAGATCAGTGCTCGCTG } \\
\text { AGCCATG }\end{array}$ & \\
\hline UAS-mCherry::pio-for & $\begin{array}{l}\text { GAATTCATGAAGACAGGC } \\
\text { ACTCGAATGGACGCTTTCC } \\
\text { ACACGGCGCTGCACTTAAT } \\
\text { CACAATCGCAGCTCTGACG } \\
\text { ACG }\end{array}$ & \multirow{4}{*}{$\begin{array}{l}\text { Assembly of mCherry } \\
\text { encoding sequence with pio } \\
\text { ORF in pJET1.2 via EcoRI / } \\
\text { XhoI and subsequent cloning } \\
\text { of mCherry::pio into } p U A S T \\
\text { via EcoRI / XbaI }\end{array}$} \\
\hline UAS-mCherry::pio-rev & $\begin{array}{l}\text { CTCGAGGCCGCCTTTGTAA } \\
\text { AGCTCATCC }\end{array}$ & \\
\hline UAS-pio-ORF-for & $\begin{array}{l}\text { CTCGAGCCAACGGCAATG } \\
\text { AAAGATGCCC }\end{array}$ & \\
\hline UAS-pio-ORF-rev & $\begin{array}{l}\text { TCTAGATTAGCTGCTGTGC } \\
\text { GAGAAG }\end{array}$ & \\
\hline UAS-Flag::pioR167A-for & $\begin{array}{l}\text { GTGCGTACAAGAGTGCCG } \\
\text { ACGCAGCCATG }\end{array}$ & \multirow{12}{*}{$\begin{array}{l}\text { In vitro mutagenesis of } \\
\text { Flag::pio to substitute either } \\
\text { one of eleven arginine or } \\
\text { lysine codons with alanine } \\
\text { codons in the region that } \\
\text { encodes the ZPD linker } \\
\text { domain and subsequent } \\
\text { cloning into } p U A S T \text { via } \\
\text { Gibson assembly (each } \\
\text { - forward primer was used } \\
\text { with UAS-rev primer and } \\
\text { each reverse primer with } \\
\text { - UAS-for primer in PCRs) }\end{array}$} \\
\hline UAS-Flag::pioR167A-rev & $\begin{array}{l}\text { CATGGCTGCGTCGGCACT } \\
\text { CTTGTACGCAC }\end{array}$ & \\
\hline UAS-Flag::pioK172A-for & $\begin{array}{l}\text { GTCGGGACGCAGCCATGG } \\
\text { CGCCCAAAAAGTACCTGC } \\
\text { GCAAGCACGC }\end{array}$ & \\
\hline UAS-Flag::pioK172A-rev & $\begin{array}{l}\text { GCGTGCTTGCGCAGGTAC } \\
\text { TTTTTGGGCGCCATGGCTG } \\
\text { CGTCCCGAC }\end{array}$ & \\
\hline UAS-Flag::pioK174A-for & $\begin{array}{l}\text { GTCGGGACGCAGCCATGA } \\
\text { AGCCCGCAAAGTACCTGC } \\
\text { GCAAGCACGC }\end{array}$ & \\
\hline UAS-Flag::pioK174A-rev & $\begin{array}{l}\text { GCGTGCTTGCGCAGGTAC } \\
\text { TTTGCGGGCTTCATGGCTG } \\
\text { CGTCCCGAC }\end{array}$ & \\
\hline UAS-Flag::pioK175A-for & $\begin{array}{l}\text { GTCGGGACGCAGCCATGA } \\
\text { AGCCCAAAGCGTACCTGC } \\
\text { GCAAGCACGC }\end{array}$ & \\
\hline UAS-Flag::pioK175A-rev & $\begin{array}{l}\text { GCGTGCTTGCGCAGGTAC } \\
\text { GCTTTGGGCTTCATGGCTG } \\
\text { CGTCCCGAC }\end{array}$ & \\
\hline UAS-Flag::pioR178A-for & $\begin{array}{l}\text { CCCAAAAAGTACCTGGCCA } \\
\text { AGCACGCCCAGAAGCCAC }\end{array}$ & \\
\hline UAS-Flag::pioR178A-rev & $\begin{array}{l}\text { GTGGCTTCTGGGCGTGCTT } \\
\text { GGCCAGGTACTTTTTGGG }\end{array}$ & \\
\hline UAS-Flag::pioK179A-for & $\begin{array}{l}\text { CCCAAAAAGTACCTGCGC } \\
\text { GCACACGCCCAGAAGCCA } \\
\text { C }\end{array}$ & \\
\hline UAS-Flag::pioK179A-rev & $\begin{array}{l}\text { GTGGCTTCTGGGCGTGTG } \\
\text { CGCGCAGGTACTTTTTGGG }\end{array}$ & \\
\hline
\end{tabular}




\begin{tabular}{|c|c|c|}
\hline UAS-Flag::pioR187A-for & $\begin{array}{l}\text { CACAGGCCTTCGCCAGCG } \\
\text { ATGATCGG }\end{array}$ & \\
\hline UAS-Flag::pioR187A-rev & $\begin{array}{l}\text { CCGATCATCGCTGGCGAA } \\
\text { GGCCTGTG }\end{array}$ & \\
\hline UAS-Flag::pioR191A-for & $\begin{array}{l}\text { CAGCGATGATCTGCGGGA } \\
\text { GTACGGA }\end{array}$ & \\
\hline UAS-Flag::pioR191A-rev & $\begin{array}{l}\text { TCCGTACTCCCGCAGATCA } \\
\text { TCGCTG }\end{array}$ & \\
\hline UAS-Flag::pioR192A-for & $\begin{array}{l}\text { CAGCGATGATCGGGCCGA } \\
\text { GTACGGAG }\end{array}$ & \\
\hline UAS-Flag::pioR192A-rev & $\begin{array}{l}\text { CTCCGTACTCGGCCCGATC } \\
\text { ATCGCTG }\end{array}$ & \\
\hline UAS-Flag::pioR196A-for & $\begin{array}{l}\text { GGAGTACGGAGCATCGCT } \\
\text { GGACAAGCAG }\end{array}$ & \\
\hline UAS-Flag::pioR196A-rev & $\begin{array}{l}\text { CCAGCGATGCTCCGTACTC } \\
\text { CCGCCGATC }\end{array}$ & \\
\hline UAS-Flag::pioK200A-for & $\begin{array}{l}\text { GACGATCGCTGGACGCAC } \\
\text { AGCAGGACGACG }\end{array}$ & \\
\hline UAS-Flag::pioK200A-rev & $\begin{array}{l}\text { CGTCGTCCTGCTGTGCGTC } \\
\text { CAGCGATCGTC }\end{array}$ & \\
\hline Strep::TGFBR3::GFP1-for & $\begin{array}{l}\text { CTGAATAGGGAATTGGGA } \\
\text { ATTCATGACTTCCCATTAT } \\
\text { G }\end{array}$ & \multirow{4}{*}{$\begin{array}{l}\text { Cloning of } \\
\text { Strep::TGFBR3::GFP into } \\
p U A S T \text { via Gibson assembly }\end{array}$} \\
\hline $\begin{array}{l}\text { Strep::TGFBR3::GFP1- } \\
\text { rev }\end{array}$ & $\begin{array}{l}\text { CACCGCTGCCACCTCCTGA } \\
\text { TCCGCCACCCTTTTCAAAC } \\
\text { TGCGGATGACTCCATGCAC } \\
\text { TTTGCACCTCTTCTGGCTC } \\
\text { TC }\end{array}$ & \\
\hline Strep::TGFBR3::GFP2-for & $\begin{array}{l}\text { ATCAGGAGGTGGCAGCGG } \\
\text { TGGAAGTGCATGGAGCCA } \\
\text { TCCCCAATTCGAGAAGGG } \\
\text { GAGCGTGGATATTGCCCT } \\
\text { G }\end{array}$ & \\
\hline $\begin{array}{l}\text { Strep::TGFBR3::GFP2- } \\
\text { rev }\end{array}$ & $\begin{array}{l}\text { TCACCATACCGCCGCTAGC } \\
\text { GGCCGTGCTGCTGCTG }\end{array}$ & \\
\hline UAS-for & $\begin{array}{l}\text { ACTGAAATCTGCCAAGAAG } \\
\text { TA }\end{array}$ & Sequencing and cloning \\
\hline UAS-rev & AGAAGTAAGGTTCCTTCAC & Sequencing and cloning \\
\hline pJet1.2-for & $\begin{array}{l}\text { CGACTCACTATAGGGAGA } \\
\text { GCGGC }\end{array}$ & Sequencing \\
\hline pJet1.2-rev & $\begin{array}{l}\text { AAGAACATCGATTTTCCAT } \\
\text { GGCAG }\end{array}$ & Sequencing \\
\hline
\end{tabular}


Table 7: List of plasmids

\begin{tabular}{|c|c|c|}
\hline Identifier & purpose & source \\
\hline pJet1.2 & Cloning of PCR amplicons & $\begin{array}{l}\text { Thermo } \\
\text { Fisher } \\
\text { Scientific }\end{array}$ \\
\hline$p U A S T$ & $\begin{array}{l}\text { Generation of transgenic flies for UAS- } \\
\text { controlled expression of ORFs; }\end{array}$ & $\begin{array}{l}\text { Brand and } \\
\text { Perrimon } \\
1993\end{array}$ \\
\hline$p B F_{V}-U 6.2$ & Expression of sgRNAs & $\begin{array}{l}\text { Kondo and } \\
\text { Ueda } 2013\end{array}$ \\
\hline$p B F_{V}-U 6.2 B$ & Simultaneous expression of two sgRNAs & $\begin{array}{l}\text { Kondo and } \\
\text { Ueda } 2013\end{array}$ \\
\hline$p G X$-attP & $\begin{array}{l}\text { Targeted genomic insertion of an attP site } \\
\text { via homology directed repair }\end{array}$ & $\begin{array}{l}\text { DGRC; } \\
\text { Huang et al. } \\
2009\end{array}$ \\
\hline$p H D$-DsRed-attP & $\begin{array}{l}\text { Targeted genomic insertion of an attP site } \\
\text { via homology directed repair }\end{array}$ & $\begin{array}{l}\text { DGRC; } \\
\text { Gratz et al. } \\
2014\end{array}$ \\
\hline pHD-ScarlessDsRed & $\begin{array}{l}\text { "Scarless" genome engineering via } \\
\text { homology directed repair }\end{array}$ & $\begin{array}{l}\text { DGRC; } \\
\text { Gratz et al. } \\
2014\end{array}$ \\
\hline$p G E$-attB & $\begin{array}{l}\text { Landing of constructs in attP alleles via } \\
\text { ФC31 integrase }\end{array}$ & $\begin{array}{l}\text { DGRC; } \\
\text { Huang et al. } \\
2009\end{array}$ \\
\hline actin5C-Gal4 & $\begin{array}{l}\text { Activating expression of UAS-constructs in } \\
\text { cultured cells }\end{array}$ & $\begin{array}{l}\text { Usui et al. } \\
1999\end{array}$ \\
\hline pOT2-LD47230 & tpr cDNA; PCR template & DGRC \\
\hline pOT2-LD43328 & lint cDNA; PCR template & DGRC \\
\hline pOT2-GHO4558 & pio cDNA; PCR template & DGRC \\
\hline $\begin{array}{l}\text { pCMV6-XL4- } \\
\text { NM_O21978 }\end{array}$ & Human matriptase cDNA; PCR template & OriGene \\
\hline $\begin{array}{l}p C M V 6-X L 5- \\
N M \_002773\end{array}$ & Human prostasin cDNA; PCR template & OriGene \\
\hline$p M D-T G F b R 3$ & Human TGFBR3 cDNA; PCR template & $\begin{array}{l}\text { Sino } \\
\text { Biological }\end{array}$ \\
\hline$p B F_{V}-U 6.2-N p-s g R N A 1$ & CRISPR/Cas9 mediated mutagenesis of $N p$ & This thesis \\
\hline$p B F_{V}-U 6.2-N p-s g R N A 2$ & $\begin{array}{l}\text { CRISPR/Cas } 9 \text { mediated editing of } N p \text { via } \\
\text { homology directed repair }\end{array}$ & This thesis \\
\hline$p B F_{V-U 6.2-t p r-s g R N A 1}$ & CRISPR/Cas9 mediated mutagenesis of $t p r$ & This thesis \\
\hline $\begin{array}{l}p B F_{V-U 6.2 B-t p r-} \\
\text { sgRNA1-sgRNA2 }\end{array}$ & $\begin{array}{l}\text { CRISPR/Cas9 mediated editing of tpr via } \\
\text { homology directed repair }\end{array}$ & This thesis \\
\hline$p B F_{V-}$ U6.2-lint-sgRNA1 & $\begin{array}{l}\text { CRISPR/Cas9 mediated mutagenesis of } \\
\text { lint }\end{array}$ & This thesis \\
\hline
\end{tabular}




\begin{tabular}{|c|c|c|}
\hline$p B F_{V}-U 6.2-p i o-s g R N A 1$ & $\begin{array}{l}\text { CRISPR/Cas } 9 \text { mediated editing of pio via } \\
\text { homology directed repair }\end{array}$ & This thesis \\
\hline$p G X$-attP-Np-HAs & $\begin{array}{l}\text { Exchange of } N p \text { genomic region with an } \\
\text { attP landing site via homology directed } \\
\text { repair }\end{array}$ & This thesis \\
\hline $\begin{array}{l}\text { pHD-DsRed-attP-tpr- } \\
H A s\end{array}$ & $\begin{array}{l}\text { Exchange of } t p r \text { genomic region with an } \\
\text { attP landing site via homology directed } \\
\text { repair }\end{array}$ & This thesis \\
\hline $\begin{array}{l}\text { pHD-ScarlessDsRed- } \\
\text { mCherry::pio-HAs }\end{array}$ & $\begin{array}{l}\text { Insertion of a mCherry encoding sequence } \\
\text { into pio via homology directed repair }\end{array}$ & This thesis \\
\hline$p G E$-attB-Np-rescue & $\begin{array}{l}\text { Landing of } N p \text { genomic rescue construct in } \\
N p^{\text {attP }} \text { allele }\end{array}$ & This thesis \\
\hline$p G E-a t t B-N p:: G F P$ & $\begin{array}{l}\text { Landing of } N p:: G F P \text { construct in } N p^{\text {att } P} \\
\text { allele }\end{array}$ & This thesis \\
\hline$p G E$-attB-Np::Strep & $\begin{array}{l}\text { Landing of } N p:: \text { Strep construct in } N p^{\text {attP }} \\
\text { allele }\end{array}$ & This thesis \\
\hline$p G E$-attB-Np $p^{S 990 A}::$ Strep & $\begin{array}{l}\text { Landing of } N p^{S 990 A}:: \text { Strep construct in } \\
N p^{\text {attP }} \text { allele }\end{array}$ & This thesis \\
\hline$p G E$-attB-tpr-rescue & $\begin{array}{l}\text { Landing of } t p r \text { genomic rescue construct in } \\
t p r^{a t t P} \text { allele }\end{array}$ & This thesis \\
\hline pGE-attB-tpr::mCherry & $\begin{array}{l}\text { Landing of tpr::mCherry construct in } \\
t p r^{a t t P} \text { allele }\end{array}$ & This thesis \\
\hline$p U A S T-N p$ & UAS-controlled expression of $\mathrm{Np}$ & This thesis \\
\hline$p U A S T-N p^{S 990 A}$ & UAS-controlled expression of $\mathrm{Np}^{\mathrm{S} 990 \mathrm{~A}}$ & This thesis \\
\hline$p U A S T-N p:: G F P$ & UAS-controlled expression of Np::GFP & This thesis \\
\hline pUAST-Np::Strep & UAS-controlled expression of Np::Strep & This thesis \\
\hline$p U A S T-N p^{S 990 A}::$ Strep & UAS-controlled expression of $\mathrm{Np}^{\mathrm{S} 990 \mathrm{~A}}::$ Strep & This thesis \\
\hline$p U A S T-t p r$ & UAS-controlled expression of Tpr & This thesis \\
\hline pUAST-tpr::Flag & UAS-controlled expression of Tpr::Flag & This thesis \\
\hline$p U A S T-$ tpr $^{Z L}:: F l a g$ & UAS-controlled expression of $\mathrm{Tpr}^{\mathrm{ZL}}::$ Flag & This thesis \\
\hline$p U A S T$-lint & UAS-controlled expression of Lint & This thesis \\
\hline pUAST-matriptase & $\begin{array}{l}\text { UAS-controlled expression of human } \\
\text { matriptase }\end{array}$ & This thesis \\
\hline pUAST-matriptase $\mathrm{S}^{S 05 \mathrm{~A}}$ & $\begin{array}{l}\text { UAS-controlled expression of } \\
\text { matriptase } \\
\text { S805A }\end{array}$ & This thesis \\
\hline $\begin{array}{l}\text { pUAST- } \\
\text { matriptase::Strep }\end{array}$ & $\begin{array}{l}\text { UAS-controlled expression of } \\
\text { matriptase::Strep }\end{array}$ & This thesis \\
\hline $\begin{array}{l}\text { pUAST- } \\
\text { matriptase } \\
\text { S805A }:: S t r e p\end{array}$ & $\begin{array}{l}\text { UAS-controlled expression of } \\
\text { matriptase }{ }^{\mathrm{S} 805 \mathrm{~A}}: \text { :Strep }\end{array}$ & This thesis \\
\hline pUAST-prostasin & $\begin{array}{l}\text { UAS-controlled expression of human } \\
\text { prostasin }\end{array}$ & This thesis \\
\hline
\end{tabular}




\begin{tabular}{|c|c|c|}
\hline$p U A S T-H A I-1$ & UAS-controlled expression of human HAI-1 & This thesis \\
\hline pUAST-HAI-2 & UAS-controlled expression of human HAI-2 & This thesis \\
\hline pUAST-mCherry::pio & UAS-controlled expression of mCherry::pio & This thesis \\
\hline pUAST-Flag::pio & UAS-controlled expression of Flag::pio & This thesis \\
\hline pUAST-Flag::pio ${ }^{R 167 A}$ & UAS-controlled expression of Flag::pio ${ }^{\text {R167A }}$ & This thesis \\
\hline pUAST-Flag::pio ${ }^{K 172 A}$ & UAS-controlled expression of Flag::pio ${ }^{\mathrm{K} 172 \mathrm{~A}}$ & This thesis \\
\hline pUAST-Flag::pio ${ }^{K 174 A}$ & UAS-controlled expression of Flag::pio ${ }^{\mathrm{K} 174 \mathrm{~A}}$ & This thesis \\
\hline pUAST-Flag:::pio ${ }^{K 175 A}$ & UAS-controlled expression of Flag::pio ${ }^{\mathrm{K} 175 \mathrm{~A}}$ & This thesis \\
\hline pUAST-Flag::pio ${ }^{R 178 A}$ & UAS-controlled expression of Flag::pio ${ }^{\text {R178A }}$ & This thesis \\
\hline pUAST-Flag::pio ${ }^{K 179 A}$ & UAS-controlled expression of Flag::pio ${ }^{\mathrm{K} 179 \mathrm{~A}}$ & This thesis \\
\hline pUAST-Flag::pio ${ }^{R 187 A}$ & UAS-controlled expression of Flag::pio ${ }^{\mathrm{R} 187 \mathrm{~A}}$ & This thesis \\
\hline pUAST-Flag::pio ${ }^{R 191 A}$ & UAS-controlled expression of Flag::pio ${ }^{\mathrm{R} 191 \mathrm{~A}}$ & This thesis \\
\hline pUAST-Flag::pio ${ }^{R 192 A}$ & UAS-controlled expression of Flag::pio ${ }^{\mathrm{R} 192 \mathrm{~A}}$ & This thesis \\
\hline$p U A S T-F l a g:: p^{2} o^{R 196 A}$ & UAS-controlled expression of Flag::pio ${ }^{\text {R196A }}$ & This thesis \\
\hline pUAST-Flag::pio ${ }^{K 200 A}$ & UAS-controlled expression of Flag::pio ${ }^{\mathrm{K} 200 \mathrm{~A}}$ & This thesis \\
\hline $\begin{array}{l}\text { pUAST- } \\
\text { Strep::TGFBR3::GFP }\end{array}$ & $\begin{array}{l}\text { UAS-controlled expression of } \\
\text { Strep::TGFBR3::GFP }\end{array}$ & This thesis \\
\hline$p U A S T-G F P$ & UAS-controlled expression of GFP & $\begin{array}{l}\text { Reinhard } \\
\text { Schuh }^{1}\end{array}$ \\
\hline
\end{tabular}

\subsubsection{Drosophila melanogaster cell lines and Escherichia coli strains}

Table 8: List of Drosophila melanogaster cell lines

\begin{tabular}{lll}
\hline Identifier & Source \\
\hline S2R+ & DGRC \\
\hline Kc167 & DGRC \\
\hline
\end{tabular}

Table 9: List of Escherichia coli strains

\begin{tabular}{|c|c|c|}
\hline Identifier & Genotype & Source \\
\hline TOP10 & $\begin{array}{l}\text { F- } m c r \mathrm{~A} \Delta(\text { mrr- } h s d \mathrm{RMS}-\text { mcrBC) } \Phi 80 \text { lacZ } \Delta \mathrm{M} 15 \\
\Delta \text { lacX74 recA1 araD139 } \Delta(\text { araleu }) 7697 \text { galU } \\
\text { galK rpsL (StrR) endA1 nupG }\end{array}$ & $\begin{array}{l}\text { Thermo Fisher } \\
\text { Scientific }\end{array}$ \\
\hline DH5a & $\begin{array}{l}\text { F- } \text { \$80lac ZMD15 D (lacZYA-argF) U169 recA1 } \\
\text { endA1 hsdR17 (rK-, mK+) phoA supE44 } \mathrm{A}-\text { thi-1 } \\
\text { gyrA46 relA1 }\end{array}$ & $\begin{array}{l}\text { Thermo Fisher } \\
\text { Scientific }\end{array}$ \\
\hline
\end{tabular}




\subsubsection{Drosophila melanogaster strains}

Table 10: List of Drosophila melanogaster strains

\begin{tabular}{|c|c|c|}
\hline Genotype & Description & Source \\
\hline$W^{1118}$ & Wild-type & $\begin{array}{l}\text { Reinhard } \\
\text { Schuh }^{1}\end{array}$ \\
\hline Oregon- $R-C$ & Wild-type & BDSC \\
\hline$w^{*} ; s n a^{S c o} / C y O, d f d-e Y F P$ & $\begin{array}{l}2^{\text {nd }} \text { chromosome balancer strain; } d f d- \\
\text { eYFP fluorescent marker is visible in } \\
\text { embryos }\end{array}$ & BDSC \\
\hline$w^{*} ; w g^{S p} / C y O, d f d-e Y F P$ & $\begin{array}{l}2^{\text {nd }} \text { chromosome balancer strain; } d f d- \\
\text { eYFP fluorescent marker is visible in } \\
\text { embryos }\end{array}$ & $\mathrm{BDSC}$ \\
\hline$w^{*} ; w g^{S p} / C y O$ & $2^{\text {nd }}$ chromosome balancer strain & $\begin{array}{l}\text { Reinhard } \\
\text { Schuh }^{1}\end{array}$ \\
\hline$w^{*} ; ; D r^{1} / T M 3, S b^{1}, d f d-e Y F P$ & $\begin{array}{l}3^{\text {rd }} \text { chromosome balancer strain; } d f d- \\
\text { eYFP fluorescent marker is visible in } \\
\text { embryos }\end{array}$ & $\mathrm{BDSC}$ \\
\hline $\begin{array}{l}w^{*} ; w g^{S p} / C y O, d f d-e Y F P \\
D^{1} / T M 3, S b^{1}\end{array}$ & $\begin{array}{l}2^{\text {nd }} \text { and } 3^{\text {rd }} \text { chromosomes balancer } \\
\text { strain; } d f d \text {-eYFP fluorescent marker is } \\
\text { visible in embryos }\end{array}$ & $\begin{array}{l}\text { Reinhard } \\
\text { Schuh }^{1}\end{array}$ \\
\hline$W^{*} ; P\{G a l 4-b t l\} / P\{G a l 4-b t 1\}$ & $\begin{array}{l}\text { btl-Gal4; Tracheal system specific } \\
\text { Gal4 driver }\end{array}$ & $\begin{array}{l}\text { Ohshiro } \\
\text { and Saigo, } \\
1997\end{array}$ \\
\hline$W^{1118} ; P\{G D 13443\}$ v23381/TM3 & $\begin{array}{l}\text { Expression of dsRNA for RNAi of } N p \\
\text { (CG34350) under control of UAS }\end{array}$ & VDRC \\
\hline $\begin{array}{l}W^{*} ; P\{K K 107228\} V I E- \\
260 B / P\{K K 107228\} V I E-260 B\end{array}$ & $\begin{array}{l}\text { Expression of dsRNA for RNAi of } t p r \\
(C G 4386) \text { under control of UAS }\end{array}$ & VDRC \\
\hline $\begin{array}{l}W^{*} ; P\{K K 105169\} V I E- \\
260 B / P\{K K 105169\} V I E-260 B\end{array}$ & $\begin{array}{l}\text { Expression of dsRNA for RNAi of lint } \\
\text { (CG8213) under control of UAS }\end{array}$ & VDRC \\
\hline$W^{*} ; N^{P 6} / C y O, d f d-e Y F P$ & $N p$ mutant allele & This thesis \\
\hline$w^{*} ; \mathrm{Np}^{C 2} / \mathrm{Cy} O$, dfd-eYFP & $N p$ mutant allele & This thesis \\
\hline$w^{*} ; N p^{a t t P} / C y O, d f d-e Y F P$ & $\begin{array}{l}\text { Deletion of } N p \text { exons } 7-11 \text { by } \\
\text { replacement with an attP site }\end{array}$ & This thesis \\
\hline$W^{*} ; N p^{a t t B, L o x} / N p^{a t t B, L o x}$ & $\begin{array}{l}\text { Rescue of } N p^{a t t P} \text { allele by knock-in of } \\
N p \text { exons } 7-11\end{array}$ & This thesis \\
\hline w; Np::eGFP/CyO, dfd-eYFP & $\begin{array}{l}\text { Endogenous expression of Np::eGFP } \\
\text { fusion protein }\end{array}$ & This thesis \\
\hline$w^{*} ; N p:: S t r e p / C y O, d f d-e Y F P$ & $\begin{array}{l}\text { Endogenous expression of } \mathrm{Np}: \text { :Strep } \\
\text { fusion protein }\end{array}$ & This thesis \\
\hline
\end{tabular}




\begin{tabular}{|c|c|c|}
\hline $\begin{array}{l}W^{*} ; p^{S 990 A}:: S t r e p / C y O, d f d- \\
e Y F P\end{array}$ & $\begin{array}{l}\text { Endogenous expression of } \\
\text { catalytically inactive } \mathrm{Np}^{\mathrm{S} 990 \mathrm{~A}}: \text { :Strep } \\
\text { fusion protein }\end{array}$ & This thesis \\
\hline$w^{*} ; t p r^{D 1} / C y O, d f d-e Y F P$ & tpr mutant allele & This thesis \\
\hline$w^{*} ; t p r^{F 1} / C y O, d f d-e Y F P$ & tpr mutant allele & This thesis \\
\hline$w^{*} ; t p r^{a t t P} / C y O, d f d-e Y F P$ & $\begin{array}{l}\text { Deletion of tpr genomic region by } \\
\text { replacement with an attP site }\end{array}$ & This thesis \\
\hline$w^{*} ; t p r^{a t t B, L o x} / t p r^{a t t B, L o x}$ & $\begin{array}{l}\text { Rescue of } t p r^{a t t P} \text { allele by knock-in of } \\
\text { tpr genomic region }\end{array}$ & This thesis \\
\hline $\begin{array}{l}\text { W* tpr::mCherry/tpr::mCher- } \\
\text { ry }\end{array}$ & $\begin{array}{l}\text { Endogenous expression of } \\
\text { Tpr::mCherry fusion protein }\end{array}$ & This thesis \\
\hline$w^{*} ; \operatorname{lin} t^{C 1} / C y O, d f d-e Y F P$ & lint mutant allele & This thesis \\
\hline$w^{*} ; \operatorname{lin} t^{K 7} / C y O, d f d-e Y F P$ & lint mutant allele & This thesis \\
\hline $\begin{array}{l}W^{*} ; \mathrm{Np}^{22 F}, \operatorname{lint} t^{22 F} / C y O, d f d- \\
e Y F P\end{array}$ & $N p$, lint double mutant allele & This thesis \\
\hline $\begin{array}{l}w^{*} ; m \text { Cherry::pio/CyO, dfd- } \\
\text { eYFP }\end{array}$ & $\begin{array}{l}\text { Endogenous expression of } \\
\text { mCherry::Pio fusion protein }\end{array}$ & This thesis \\
\hline$W^{1118} ; D f(2 R) B S C 271 / C y O$ & $\begin{array}{l}\text { Deficiency chromosome in which the } \\
\text { region } 44 \mathrm{~F} 12-45 \mathrm{~A} 12 \text { is deleted, } \\
\text { including } \mathrm{Np} \text { and lint }\end{array}$ & BDSC \\
\hline$W^{1118} ; D f(2 R) B S C 664 / S M 6 a$ & $\begin{array}{l}\text { Deficiency chromosome in which the } \\
\text { region } 57 \mathrm{D} 12-58 \mathrm{~A} 3 \text { is deleted, } \\
\text { including tpr }\end{array}$ & BDSC \\
\hline $\begin{array}{l}W^{1118} ; ; P\{G M R 34 G 06- \\
\text { GAL4\}attP2/P\{GMR34G06- } \\
\text { GAL4\}attP2 }\end{array}$ & $\begin{array}{l}A b d B \text {-Gal4; Ectodermal tissues in } \\
\text { abdominal segments A8-A14 specific } \\
\text { Gal4 driver }\end{array}$ & BDSC \\
\hline $\begin{array}{l}W^{1118} ; P b a c\{681 . P . F S V S-1\} d p y \\
\text { CPTIо01769/Pbac }\{681 . P . F S V S- \\
1\} d p y \text { CPTIO01769 }\end{array}$ & $\begin{array}{l}d p y: \text { YFP; Endogenous expression of } \\
\text { Dpy::YFP fusion protein }\end{array}$ & DGGR \\
\hline $\begin{array}{l}W^{1118} ; \text { Pbac }\{681 . P . F S V S- \\
1\} C G 8213^{C P T I 001902} / P B a c\{681 . P . \\
F S V S-1\} C G 8213^{C P T I 001902}\end{array}$ & $\begin{array}{l}\text { lint::YFP; Endogenous expression of } \\
\text { Lint::YFP fusion protein }\end{array}$ & DGGR \\
\hline $\begin{array}{l}w^{*} ; ; P\{U A S p-G F P S 65 C \text {-al- } \\
\text { phaTub84B\}3/TM3, Sb }\end{array}$ & $\begin{array}{l}\text { Expression of a-tub::GFP under } \\
\text { control of UAS }\end{array}$ & BDSC \\
\hline$W^{*} ; P\{U A S-N p\} / P\{U A S-N p\}$ & $\begin{array}{l}\text { Expression of } \mathrm{Np} \text { under control of } \\
\text { UAS }\end{array}$ & $\begin{array}{l}\text { Reinhard } \\
\text { Schuh }^{1}\end{array}$ \\
\hline$w^{*} ; ; P\{U A S-N p\} / P\{U A S-N p\}$ & $\begin{array}{l}\text { Expression of } \mathrm{Np} \text { under control of } \\
\text { UAS }\end{array}$ & $\begin{array}{l}\text { Reinhard } \\
\text { Schuh }^{1}\end{array}$ \\
\hline $\begin{array}{l}W^{*} ; P\{U A S- \\
N p:: e G F P\} / P\{U A S-N p:: e G F P\}\end{array}$ & $\begin{array}{l}\text { Expression of Np::eGFP under control } \\
\text { of UAS }\end{array}$ & This thesis \\
\hline $\begin{array}{l}w^{*} ; ; P\{U A S- \\
N p:: e G F P\} / P\{U A S-N p:: e G F P\}\end{array}$ & $\begin{array}{l}\text { Expression of Np::eGFP under control } \\
\text { of UAS }\end{array}$ & This thesis \\
\hline
\end{tabular}




\begin{tabular}{|c|c|c|}
\hline $\begin{array}{l}W^{*} ; P\left\{U A S-N p^{S 990 A}\right\} / P\{U A S- \\
\left.N p^{S 990 A}\right\}\end{array}$ & $\begin{array}{l}\text { Expression of } \mathrm{Np}^{\mathrm{S} 990 \mathrm{~A}} \text { under control of } \\
\text { UAS }\end{array}$ & This thesis \\
\hline $\begin{array}{l}W^{*} ; ; P\left\{U A S-N p^{S 990 A}\right\} / P\{U A S- \\
\left.N p^{S 990 A}\right\}\end{array}$ & $\begin{array}{l}\text { Expression of } \mathrm{Np}^{\mathrm{S} 990 \mathrm{~A}} \text { under control of } \\
\text { UAS }\end{array}$ & This thesis \\
\hline$w^{*} ; P\{U A S-t p r\} / P\{U A S-t p r\}$ & $\begin{array}{l}\text { Expression of Tpr under control of } \\
\text { UAS }\end{array}$ & This thesis \\
\hline$w^{*} ; ; P\{U A S-t p r\} / P\{U A S-t p r\}$ & $\begin{array}{l}\text { Expression of Tpr under control of } \\
\text { UAS }\end{array}$ & This thesis \\
\hline$w^{*}, P\{U A S-\operatorname{lin} t\}$ & $\begin{array}{l}\text { Expression of Lint under control of } \\
\text { UAS }\end{array}$ & This thesis \\
\hline $\begin{array}{l}W^{*} ; P\{U A S- \\
\text { matriptase }\} / P\{U A S-m a t r i p- \\
\text { tase }\}\end{array}$ & $\begin{array}{l}\text { Expression of human matriptase } \\
\text { under control of UAS }\end{array}$ & This thesis \\
\hline $\begin{array}{l}W^{*} ; ; P\{U A S- \\
\text { matriptase }\} / P\{U A S-\text { matrip- } \\
\text { tase }\}\end{array}$ & $\begin{array}{l}\text { Expression of human matriptase } \\
\text { under control of UAS }\end{array}$ & This thesis \\
\hline $\begin{array}{l}W^{*} ; P\{U A S-\text { prostasin }\} / P\{U A S- \\
\text { prostasin }\}\end{array}$ & $\begin{array}{l}\text { Expression of human prostasin under } \\
\text { control of UAS }\end{array}$ & This thesis \\
\hline $\begin{array}{l}W^{*} ; ; P\{U A S \text {-prostasin }\} / \\
P\{U A S \text {-prostasin }\}\end{array}$ & $\begin{array}{l}\text { xpression of human prostasin under } \\
\text { ntrol of UAS }\end{array}$ & This thesis \\
\hline $\begin{array}{l}W^{*} ; P\{U A S-H A I-1\} / P\{U A S- \\
H A I-1\}\end{array}$ & $\begin{array}{l}\text { xpression of human HAI-1 under } \\
\text { ntrol of UAS }\end{array}$ & This thesis \\
\hline $\begin{array}{l}W^{*} ; ; P\{U A S-H A I-1\} / P\{U A S- \\
H A I-1\}\end{array}$ & $\begin{array}{l}\text { Expression of human HAI-1 under } \\
\text { control of UAS }\end{array}$ & This thesis \\
\hline $\begin{array}{l}W^{*} ; P\{U A S-H A I-2\} / P\{U A S- \\
H A I-2\}\end{array}$ & $\begin{array}{l}\text { xpression of human HAI-2 under } \\
\text { ontrol of UAS }\end{array}$ & This thesis \\
\hline $\begin{array}{l}W^{*} ; ; P\{U A S-H A I-2\} / P\{U A S- \\
H A I-2\}\end{array}$ & $\begin{array}{l}\text { Expression of human HAI-2 under } \\
\text { control of UAS }\end{array}$ & This thesis \\
\hline $\begin{array}{l}w^{*} ; N^{P 6}, P b a c\{681 . P . F S V S- \\
1\} d p y^{C P T I 001769} / C y O, d f d-e Y F P\end{array}$ & $\begin{array}{l}\text { Endogenous expression of Dpy::YFP } \\
\text { in } N p \text { mutant embryos }\end{array}$ & This thesis \\
\hline $\begin{array}{l}w^{*} ; t^{D 1}, r^{D b a c}\{681 . P . F S V S- \\
1\} d p y^{C P T I 001769} / C y O, \text { dfd-eYFP }\end{array}$ & $\begin{array}{l}\text { Endogenous expression of Dpy::YFP } \\
\text { in tpr mutant embryos }\end{array}$ & This thesis \\
\hline $\begin{array}{l}W^{*} ; t^{F 1}, P b a c\{681 . P . F S V S- \\
1\} d p y^{C P T I 001769} / C y O, d f d-e Y F P\end{array}$ & $\begin{array}{l}\text { Endogenous expression of Dpy::YFP } \\
\text { in tpr mutant embryos }\end{array}$ & This thesis \\
\hline $\begin{array}{l}w^{*} ; \operatorname{lin} t^{C 1}, P b a c\{681 . P . F S V S- \\
1\} d p y^{C P T 1001769} / C y O, d f d-e Y F P\end{array}$ & $\begin{array}{l}\text { Endogenous expression of Dpy::YFP } \\
\text { in lint mutant embryos }\end{array}$ & This thesis \\
\hline $\begin{array}{l}w^{*} ; N^{P 6}, m \text { Cherry::pio/CyO, } \\
\text { dfd-eYFP }\end{array}$ & $\begin{array}{l}\text { Endogenous expression of } \\
\text { mCherry::Pio in } N p \text { mutant embryos }\end{array}$ & This thesis \\
\hline $\begin{array}{l}w^{*} ; \operatorname{lin} t^{C 1}, \text { mCherry::pio/CyO, } \\
\text { dfd-eYFP }\end{array}$ & $\begin{array}{l}\text { Endogenous expression of } \\
\text { mCherry::Pio in lint mutant embryos }\end{array}$ & This thesis \\
\hline $\begin{array}{l}{ }^{*} ; N^{P 6}, P\{G a l 4-b t l\} / C y O \\
d f d-e Y F P\end{array}$ & $\begin{array}{l}\text { btl-Gal4 driver in } N p \text { mutant } \\
\text { background }\end{array}$ & This thesis \\
\hline
\end{tabular}




\begin{tabular}{|c|c|c|}
\hline $\begin{array}{l}W^{*} ; N^{P 6}, P\{G a 14-b t 1\} \\
P b a c\{681 . P . F S V S-1\} d p y^{C P .} \\
\text { TIoo1769/CyO, dfd-eYFP }\end{array}$ & $\begin{array}{l}\text { btl-Gal4 driver in } N p \text { mutant } \\
\text { background with endogenous } \\
\text { expression of Dpy::YFP }\end{array}$ & This thesis \\
\hline $\begin{array}{l}W^{*} ; N^{P 6} / C y O, \text { dfd-eYFP; } \\
P\{G M R 34 G 06- \\
\text { GAL4\}attP2/P\{GMR34G06- } \\
\text { GAL4\}attP2 }\end{array}$ & $\begin{array}{l}A b d B \text {-Gal4 driver in } N p \text { mutant } \\
\text { background }\end{array}$ & This thesis \\
\hline $\begin{array}{l}W^{*} ; \operatorname{tpr}^{D 1}, P\{G a l 4-b t l\} / C y O \\
\text { dfd-eYFP }\end{array}$ & $\begin{array}{l}\text { btl-Gal } 4 \text { driver in } t p r \text { mutant } \\
\text { background }\end{array}$ & This thesis \\
\hline $\begin{array}{l}w^{*} ; \operatorname{lin} t^{C 1}, P\{G a l 4-b t l\} / C y O, \\
d f d-e Y F P\end{array}$ & $\begin{array}{l}\text { btl-Gal4 driver in lint mutant } \\
\text { background }\end{array}$ & This thesis \\
\hline $\begin{array}{l}W^{*} ; N^{C 2}, P\{U A S-N p\} / C y O \\
d f d-e Y F P\end{array}$ & $\begin{array}{l}\text { Expression of Np under control of } \\
\text { UAS in } N p \text { mutant background }\end{array}$ & This thesis \\
\hline $\begin{array}{l}W^{*} ; N p^{C 2} / C y O, \text { dfd-eYFP } \\
P\{U A S-N p\} / P\{U A S-N p\}\end{array}$ & $\begin{array}{l}\text { Expression of Np under control of } \\
\text { UAS in } N p \text { mutant background }\end{array}$ & This thesis \\
\hline $\begin{array}{l}W^{*} ; N^{C 2}, P\left\{U A S-N p^{S 990 A}\right\} / C y O, \\
\text { dfd-eYFP }\end{array}$ & $\begin{array}{l}\text { Expression of } \mathrm{Np}^{\mathrm{S} 990 \mathrm{~A}} \text { under control of } \\
\text { UAS in } N p \text { mutant background }\end{array}$ & This thesis \\
\hline $\begin{array}{l}W^{*} ; N p^{C 2} / C y O, \text { dfd-eYFP } \\
P\left\{U A S-N p^{S 990 A}\right\} / P\{U A S- \\
\left.N p^{S 990 A}\right\}\end{array}$ & $\begin{array}{l}\text { Expression of } \mathrm{Np}^{\mathrm{S} 990 \mathrm{~A}} \text { under control of } \\
\text { UAS in } N p \text { mutant background }\end{array}$ & This thesis \\
\hline $\begin{array}{l}W^{*} ; N p^{C 2}, P\{U A S- \\
N p:: e G F P\} / C y O, d f d-e Y F P\end{array}$ & $\begin{array}{l}\text { Expression of Np::eGFP under control } \\
\text { of UAS in } N p \text { mutant background }\end{array}$ & This thesis \\
\hline $\begin{array}{l}W^{*}, P\{U A S \text {-lint }\} ; N p^{C 2} / C y O \\
\text { dfd-eYFP }\end{array}$ & $\begin{array}{l}\text { Expression of Lint under control of } \\
\text { UAS in } N p \text { mutant background }\end{array}$ & This thesis \\
\hline $\begin{array}{l}W^{*} ; N^{C 2}, P\{U A S- \\
\text { matriptase } / C y O, \text { dfd-eYFP }\end{array}$ & $\begin{array}{l}\text { Expression of human matriptase } \\
\text { under control of UAS in } N p \text { mutant } \\
\text { background }\end{array}$ & This thesis \\
\hline $\begin{array}{l}W^{*} ; N^{C 2} / C y O, \text { dfd-eYFP } \\
P\{U A S-m a t r i p t a s e\} / P\{U A S- \\
\text { matriptase }\}\end{array}$ & $\begin{array}{l}\text { Expression of human matriptase } \\
\text { under control of UAS in } N p \text { mutant } \\
\text { background }\end{array}$ & This thesis \\
\hline $\begin{array}{l}W^{*} ; N p^{C 2} / C y O, d f d-e Y F P \\
P\{U A S-H A I-1\} / P\{U A S-H A I-1\}\end{array}$ & $\begin{array}{l}\text { Expression of human HAI-1 under } \\
\text { control of UAS in } N p \text { mutant } \\
\text { background }\end{array}$ & This thesis \\
\hline $\begin{array}{l}W^{*} ; N p^{C 2} / C y O, \text { dfd-eYFP } \\
P\{U A S-H A I-2\} / P\{U A S-H A I-2\}\end{array}$ & $\begin{array}{l}\text { Expression of human HAI- } 2 \text { under } \\
\text { control of UAS in } N p \text { mutant } \\
\text { background }\end{array}$ & This thesis \\
\hline $\begin{array}{l}w^{*} ; N p^{C 2}, P\{U A S- \\
\text { matriptase }\} / C y O, \text { dfd-eYFP } ; \\
P\{U A S-H A I-1\} / P\{U A S-H A I-1\}\end{array}$ & $\begin{array}{l}\text { Co-expression of human matriptase } \\
\text { and HAI- } 1 \text { under control of UAS in } \\
N p \text { mutant background }\end{array}$ & This thesis \\
\hline $\begin{array}{l}W^{*} ; N p^{C 2}, P\{U A S- \\
\text { matriptase }\} / C y O, \text { dfd-eYFP } \\
P\{U A S-H A I-2\} / P\{U A S-H A I-2\}\end{array}$ & $\begin{array}{l}\text { Co-expression of human matriptase } \\
\text { and HAI-2 under control of UAS in } \\
N p \text { mutant background }\end{array}$ & This thesis \\
\hline
\end{tabular}




\begin{tabular}{|c|c|c|}
\hline 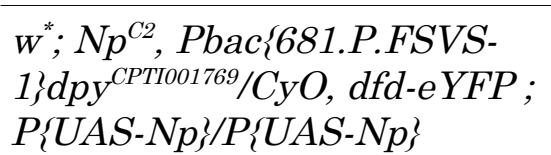 & $\begin{array}{l}\text { Expression of Np under control of } \\
\text { UAS in } N p \text { mutant background with } \\
\text { endogenous expression of Dpy::YFP }\end{array}$ & This thesis \\
\hline $\begin{array}{l}W^{*} ; N_{p}^{C 2}, P b a c\{681 . P . F S V S- \\
1\} d p y^{C P T I 001769} / C y O, d f d-e Y F P \\
P\left\{U A S-N p^{S 990 A}\right\} / P\{U A S- \\
\left.N p^{S 990 A}\right\}\end{array}$ & $\begin{array}{l}\text { Expression of } \mathrm{Np}^{\mathrm{S} 990 \mathrm{~A}} \text { under control of } \\
\text { UAS in } N p \text { mutant background with } \\
\text { endogenous expression of Dpy::YFP }\end{array}$ & This thesis \\
\hline $\begin{array}{l}W^{*} ; N^{C 2}, P\{U A S-m a t r i p t a s e\} \\
P b a c\{681 . P . F S V S-1\} d p y^{C P .} \\
\text { TI001769/CyO, dfd-eYFP }\end{array}$ & $\begin{array}{l}\text { Expression of human matriptase } \\
\text { under control of UAS in } N p \text { mutant } \\
\text { background with endogenous } \\
\text { expression of Dpy::YFP }\end{array}$ & This thesis \\
\hline $\begin{array}{l}W^{*} ; N p^{C 2}, P\{U A S-m a t r i p t a s e\} \\
P\{G a l 4-b t l\}, \\
P b a c\{681 . P . F S V S-1\} d p y^{C P-} \\
\text { TI001769/CyO,dfd-eYFP; } \\
P\{U A S-H A I-2\} / P\{U A S-H A I-2\}\end{array}$ & $\begin{array}{l}\text { Co-expression of human matriptase } \\
\text { and HAI-2 under control of UAS in } \\
N p \text { mutant background with } \\
\text { endogenous expression of Dpy::YFP }\end{array}$ & This thesis \\
\hline $\begin{array}{l}W^{*} ; N^{C 2} / C y O, d f d-e Y F P \\
P\{U A S p-G F P S 65 C-a l- \\
\text { phaTub84B\}3/TM3, Sb }\end{array}$ & $\begin{array}{l}\text { Expression of a-tub::GFP under } \\
\text { control of UAS in } N p \text { mutant } \\
\text { background }\end{array}$ & This thesis \\
\hline $\begin{array}{l}w^{*} ; t p r^{D 1}, P\{U A S-t p r\} / C y O \\
d f d-e Y F P\end{array}$ & $\begin{array}{l}\text { Expression of Tpr under control of } \\
\text { UAS in tpr mutant background }\end{array}$ & This thesis \\
\hline $\begin{array}{l}W^{*} ; t p r^{D 1} / C y O, d f d-e Y F P \\
P\{U A S-t p r\} / P\{U A S-t p r\}\end{array}$ & $\begin{array}{l}\text { Expression of Tpr under control of } \\
\text { UAS in tpr mutant background }\end{array}$ & This thesis \\
\hline $\begin{array}{l}W^{*} ; \operatorname{tpr}^{D 1}, P\{U A S- \\
\text { prostasin }\} / C y O, \text { dfd-eYFP }\end{array}$ & $\begin{array}{l}\text { Expression of human prostasin under } \\
\text { control of UAS in tpr mutant } \\
\text { background }\end{array}$ & This thesis \\
\hline $\begin{array}{l}W^{*} ; \operatorname{tpr}^{D 1} / C y O, \text { dfd-eYFP } \\
P\{U A S-\text { prostasin }\} / P\{U A S- \\
\text { prostasin }\}\end{array}$ & $\begin{array}{l}\text { Expression of human prostasin under } \\
\text { control of UAS in tpr mutant } \\
\text { background }\end{array}$ & This thesis \\
\hline $\begin{array}{l}W^{*} ; t p r^{D 1} / C y O, \text { dfd-eYFP } \\
P\{U A S-H A I-2\} / P\{U A S-H A I-2\}\end{array}$ & $\begin{array}{l}\text { Expression of human HAI- } 2 \text { under } \\
\text { control of UAS in tpr mutant } \\
\text { background }\end{array}$ & This thesis \\
\hline $\begin{array}{l}W^{*} ; \operatorname{tpr}^{D 1}, P\{U A S- \\
\text { prostasin }\} / C y O, \text { dfd-eYFP } \\
P\{U A S-H A I-2\} / P\{U A S-H A I-2\}\end{array}$ & $\begin{array}{l}\text { Co-expression of human prostasin and } \\
\text { HAI- } 2 \text { under control of UAS in tpr } \\
\text { mutant background }\end{array}$ & This thesis \\
\hline $\begin{array}{l}w^{*}, P\{U A S-\operatorname{lin} t\} / C ; \operatorname{lin} t^{K 7} / C y O, \\
d f d-e Y F P\end{array}$ & $\begin{array}{l}\text { Expression of Lint under control of } \\
\text { UAS in lint mutant background }\end{array}$ & This thesis \\
\hline $\begin{array}{l}y^{2}, c h o^{2}, v^{1} ; P\left\{\text { nos-Cas } 9, y^{+}\right. \\
\left.v^{+}\right\} 3 A / T M 6 C, S b^{1}, T b^{!}\end{array}$ & $\begin{array}{l}\text { nos-Cas9-3A; Expression of Cas } 9 \\
\text { under control of nanos promotor }\end{array}$ & NIG \\
\hline $\begin{array}{l}W^{1118} ; C y O, P\left\{\left(w^{+}\right) T u b-\right. \\
P B a c \backslash T\} 2 / w g^{S p 1}\end{array}$ & $\begin{array}{l}\text { Expression of PiggyBac transposase } \\
\text { under control of } a-t u b \text { promotor }\end{array}$ & BDRC \\
\hline $\begin{array}{l}y^{1}, w^{67 c 23} ; \mathrm{sna}^{S c o} / C y O \\
P\{C r e w\} D H 1\end{array}$ & $\begin{array}{l}\text { P1/Cre; Ubiquitous expression of Cre } \\
\text { recombinase }\end{array}$ & BDRC \\
\hline
\end{tabular}

1-Prof. Dr. Reinhard Schuh, RG Molecular Organogenesis, Max Planck Institute for Biophysical Chemistry, Göttingen, Germany 


\subsection{Fly handling, mutagenesis and transgenesis}

D. melanogaster fly stocks were kept in cylindrical plastic vials (Greiner Bio One) that were filled with fly food up to a $4^{\text {th }}$ of their hight and sealed with foam plugs. Stocks were stored at $18{ }^{\circ} \mathrm{C}$ or $22{ }^{\circ} \mathrm{C}$ and transferred to new vials every four weeks $\left(18{ }^{\circ} \mathrm{C}\right)$ or each week $\left(22{ }^{\circ} \mathrm{C}\right)$. For analysis of flies and in order to select flies according to dominant markers for crosses, flies were shortly sedated with $\mathrm{CO}_{2}$ and observed with a Stemi SV 11 stereomicroscope (Zeiss).

\subsubsection{Fly crosses and balancing of transgenic or mutant alleles}

Flies with different genotypes were crossed by selection of virgin females of one genotype and putting them into a fresh vial together with males of the other genotype. Dominant markers were used to select flies with the desired genotype in the following generations. Lethal mutant alleles and transgenic insertions that caused lethality were maintained by introduction of balancer chromosomes. Used balancer chromosomes are indicated in Table 10 for each stock. Balancer chromosomes that contain a $d f d$-eYFP transgene were used to identify homozygous mutant embryos or larvae. Flies with multiple mutant or transgenic alleles on the same chromosome were generated by selection for recombination events as follows: virgin females with exemplary allele-1 were crossed with males that carried exemplary allele-2. Next, F1 virgin females that carried allele-1 and allele-2 in trans were selected and crossed with balancer chromosome males. Finally, F2 flies that carried both alleles in cis were selected by dominant markers or subsequent crosses to test for lethality of mutant alleles.

\subsubsection{CRISPR/Cas9-mediated mutagenesis}

The CRISPR/Cas9 system was used for targeted mutagenesis. The CRISPR Optimal Target Finder tool was used to identify specific sgRNA targets in the $D$. melanogaster genome (Gratz et al. 2014). DNA oligos corresponding to the chosen sgRNA target sequences were annealed and ligated into the $p B F_{V}-U 6.2$ vector via BbsI restriction sites (Kondo and Ueda 2013). For simultaneously targeting two sgRNA recognition sites, one sgRNA target encoding annealed DNA oligo was 
ligated into the $p B F_{V}-U 6.2$ vector and the other one into the $p B F_{V}-U 6.2 B$ vector (Kondo and Ueda 2013). Subsequently, the U6.2 promotor and sgRNA target sequence of the $p B F_{V}-U 6.2$ vector were transferred to the sgRNA target sequence containing $p B F_{V}-U 6.2 B$ vector via EcoRI/NotI endonuclease restriction sites (Kondo and Ueda 2013). Vectors for sgRNA expression were injected into nos-Cas9$3 A$ embryos by BestGene. Adult flies that developed from the injected embryos were then crossed with balancer chromosome strains. Single flies of the resulting F1 generation were again crossed with balancer chromosome strains and the resulting F2 stock was analyzed regarding lethality of the putatively mutated chromosome. The regions of the genomic sgRNA target sites from the obtained lethal stocks were amplified by PCR followed by purification of the amplicon and sequencing.

\subsubsection{Targeted genome editing}

The CRISPR/Cas9 system was used for targeted replacement of genomic regions with attP landing sites or insertion of transgenes by homology-directed repair. For a schematic presentation of the genome editing steps see Figures 7, 18 and 37. Vectors for expression of sgRNAs were established as described in chapter 4.2.2. Sequences of homology arms (HAs) were amplified by PCR from genomic DNA of nos-Cas9-3A flies. HAs were then purified and cloned into the $p G X$-attP vector for editing of $N p$, into the $p H D$-DsRed-attP vector for editing of $t p r$, or into the $p H D$ ScarlessDsRed donor vector for editing of pio. A mCherry encoding sequence was inserted into the pio ORF at the 3' end of the 5' HA in the pHD-ScarlessDsRed donor vector (Fig 18). The HAs containing donor vectors together with the respective vectors for sgRNA expression, were injected into nos-Cas9-3A embryos by BestGene. Adult flies that developed from the injected embryos were crossed with $W^{*}$ flies and resulting F1 flies were selected for presence of $w^{+}$marker or 3xP3DsRed markers, respectively. Stocks were then established by crosses with balancer chromosome strains. Correct insertion of the transgenes was verified by amplification of the targeted genomic regions by PCR and subsequent purification of the amplicons and sequencing. The 3xP3-DsRed marker gene that was inserted in the pio locus was removed by crosses with the tub-PBac strain and subsequent selection of $\mathrm{F} 2$ flies that lacked the $t u b-P B a c$ balancer chromosome and the $3 x P 3-$ 
DsRed marker gene (Fig 18). The $w^{+}$marker and 3xP3-DsRed marker, respectively, that were inserted in the $N p$ and tpr loci were removed by crosses with the P1/Cre strain and subsequent selection of F2 flies that lacked the P1/Cre balancer chromosome and the marker genes (Fig 7; Fig 37).

ФC31 integrase-mediated transgenesis was used for genomic integration of rescue constructs at the generated $N p^{\text {attP }}$ and $t p r^{\text {attP }}$ landing sites. Genomic regions of $N p$ and tpr were amplified by PCR from $w^{1118}$ genomic DNA and cloned into the $p G E$ attB vector. The $N p:: G F P$ rescue construct was generated by amplification of a GFP encoding sequence with $N p$ genomic region overlapping ends by PCR and subsequent cloning into $p G E$-attB-Np via EcoRI/BmgBI restriction sites. A NheI restriction site containing linker was also introduced between the $N p$ ORF and GFP encoding sequence by this PCR. The NheI restriction site was then used to exchange the GFP encoding sequence with a synthesized TwinStrep-tag encoding sequence, and to introduce a synthesized $N p$ genomic fragment that contains a mutation in the serine 990 codon, generating the $p G E$-attB-Np::Strep and $p G E$ attB-Np$p^{S 990 A}::$ Strep vectors (fragments 2 and 4 in Appendix 7.1). The $p G E$-attBtpr::mCherry vector was generated by cloning of a synthesized tpr genomic region fragment with an introduced mCherry encoding sequence into the $p G E$-attB-tpr vector by NaeI/KpnI restriction sites (fragment 1 in Appendix 7.1). The attB site containing donor vectors together with $\Phi$ C31 integrase were injected into $N p^{\text {attP }}$ embryos or tpr ${ }^{\text {att } P}$ embryos, respectively, by BestGene. Adult flies that developed from the injected embryos were crossed with $w^{*}$ flies and resulting F1 flies were selected for presence of the $w^{+}$marker. Stocks were then established by crosses with balancer chromosome strains. Correct insertion of the constructs was verified by amplification of the respective genomic regions by PCR and subsequent purification of the amplicons and sequencing. The $w^{+}$marker was removed by crosses with the P1/Cre strain and subsequent selection of F2 flies that lacked the P1/Cre balancer chromosome and the $w^{+}$marker (Fig 7; Fig 37).

\subsubsection{Generation of UAS-responder strains}

UAS-responder strains were established for this thesis to conduct tracheal system specific rescue experiments. ORFs with attached restriction sites were generated by PCR amplification from cDNA plasmids and subsequently cloned into the 
pUAST vector. ORF amplifications of tpr and lint were done from plasmids pOT2LD47230 and pOT2-LD43328, respectively. Plasmids pCMV6-XL4-NM_021978 and pCMV6-XL5-NM_002773 were used as templates for ORF amplifications of human matriptase and human prostasin, respectively. Human HAI-1 and HAI-2 ORF sequences were synthesized with codon optimization for $D$. melanogaster according to cDNA sequences AY358969 and BC001668, respectively (fragments 9 and 10 in Appendix 7.1; Strausberg et al. 2002). For generation of $p U A S T-N p^{S 990 A}$, a synthesized fragment of the $N p$ ORF that contained a mutation in the serine 990 codon was cloned into $p U A S T-N p$ via SacII/XhoI restriction sites (fragment 6 in Appendix 7.1). The $p U A S T-N p:: G F P$ vector was established by amplification of a GFP encoding sequence with $N p$ ORF overlaps by PCR and subsequent cloning into $p U A S T-N p$ via EcoRI/XhoI restriction sites. The $p U A S T$-responder vectors were injected into embryos by BestGene for P-element-mediated germline transformation (Rubin and Spradling 1982). Adult flies that developed from the injected embryos were crossed with $w^{1118}$ flies and resulting F1 flies were selected for presence of the $W^{+}$marker. Single $W^{+}$flies were again crossed with $w^{1118}$ flies to generate strains that originated from single transgene insertion events. These strains were crossed with $2^{\text {nd }}$ and $3^{\text {rd }}$ chromosome balancer strains to determine chromosomal localization of the UAS transgenes. Finally, homozygous stocks of the UAS-responder strains were established.

\subsection{Analyses of embryos and larvae}

\subsubsection{Collection of embryos and larvae}

For collection of $D$. melanogaster embryos and larvae, flies of the desired genotype were put in cylindrical cages that were closed by applying an apple-juice agar plate to their bottom openings. A small pile of wet inactive yeast was placed in the center of the apple-juice agar plate. Egg laying took place at $25{ }^{\circ} \mathrm{C}$ for collection of embryos with pan-tracheal RNAi-mediated knock-downs. In all other cases, egglaying took place at $22{ }^{\circ} \mathrm{C}$. The apple-juice agar plates were exchanged with fresh 
plates at respective points of time to obtain embryos or larvae at certain developmental stages.

\subsubsection{Dechorionation of embryos}

Embryos were washed from the apple-juice agar plates into close-meshed nets using tab water and a brush. The embryo containing net was then submerged in bleach solution for $3 \mathrm{~min}$ for dechorionation of the embryos. Subsequently, embryos were rinsed thoroughly with tab water to wash off any residual bleach solution.

\subsubsection{In vivo analyses of tracheal liquid clearance}

For analyses of embryonic tracheal LC, embryos were collected at 20-21 hours AEL (egg-laying at $25^{\circ} \mathrm{C}$ ) or 22-23 hours AEL (egg-laying at $22^{\circ} \mathrm{C}$ ) and dechorionated. The embryos were then mounted in PBS on microscope slides and cover slips were applied. Tracheal LC was observed by bright-field light microscopy with an Axiophot microscope (Zeiss). For observation of tracheal LC in larval stages, larvae were collected from apple-juice agar plates by washing them into close-meshed nets and observed with a Stemi SV 11 stereomicroscope (Zeiss).

\subsubsection{Dextran permeability assays}

Embryos were collected and dechorionated as described in chapters 4.3.1 - 4.3.2 and placed on an apple juice agar plate in a straight row. The embryos were then attached to a microscope slide that was covered with heptane glue by carefully pressing the slide onto the embryos. For injections, embryos were covered with Voltalef $10 \mathrm{~S}$ oil. Texas Red-labeled $10 \mathrm{kDa}$ Dextran and Fluorescein-labeled 70 $\mathrm{kDa}$ dextran (Molecular Probes) were then injected into the haemocoel of embryos with a transjector 5246 (Eppendorf) and sterile Femtotips-II needles (Eppendorf). The tracheal system of the embryos was analyzed immediately after injection by confocal microscopy. 


\subsubsection{Whole-mount antibody stainings of embryos}

Embryos were collected and dechorionated as described in chapters 4.3.1 - 4.3.2. For fixation, the embryos were then transferred into scintillation vials containing 1 $\mathrm{ml}$ RNA-fix solution, $2 \mathrm{ml}$ Hepes solution and $6 \mathrm{ml}$ heptane. The vials were agitated at $250 \mathrm{rpm}$ for $20 \mathrm{~min}$ during fixation of the embryos. The lower phase fixative was subsequently removed and $8 \mathrm{ml}$ methanol were added to the vials followed by shaking at $500 \mathrm{rpm}$ for $3 \mathrm{~min}$ to detach the vitteline membrane. After removal of the supernatant, embryos were washed with methanol repeatedly and stored in methanol at $-20^{\circ} \mathrm{C}$. For antibody stainings, embryos were transferred to fresh cups and washed three times with BBT for 5 min per wash followed by treatment with blocking solution for $30 \mathrm{~min}$ to saturate unspecific immunoglobulin binding sites. Subsequently, pre-absorbed primary antibodies diluted in blocking solution were applied to the embryos and incubated over night at $4{ }^{\circ} \mathrm{C}$. Primary antibody solutions were removed on the next day and embryos were washed six times with BBT for 5 minutes per wash followed by treatment with blocking solution for $30 \mathrm{~min}$. Then, pre-absorbed secondary antibodies diluted in blocking solution were added to the embryos and incubated for 2 hours. If required, fluorophore-conjugated CBP (NEB) was added to the secondary antibody dilutions at a 1:500 dilution for staining of chitin. Finally, embryos were washed eight times with PBT for 5 minutes per wash, transferred to microscope slides and covered with Prolong mounting medium (Thermo Fisher Scientific) and a cover slip. All incubation and washing steps were performed on a rotating wheel at room temperature, if not declared otherwise.

\subsubsection{Whole-mount stainings of chitin in embryos}

Embryos were collected and dechorionated as described in chapters 4.3.1 - 4.3.2. Fixation and devittelinization was then performed as described in chapter 4.3.5. embryos were transferred to fresh cups and washed three times with BBT for 5 min per wash followed by treatment with blocking solution for $30 \mathrm{~min}$. Fluorophore-conjugated CBP (NEB) diluted 1:500 in blocking solution was added to the embryos and incubated for 3 hours. Subsequently, embryos were washed eight times with PBT for 5 minutes per wash, transferred to microscope slides and covered with Prolong mounting medium (Thermo Fisher Scientific) and a cover 
slip. All incubation and washing steps were performed on a rotating wheel at room temperature.

\subsubsection{Whole-mount in situ RNA hybridization stainings of embryos}

RNA in situ hybridization stainings of whole-mount embryos were performed with minor alterations according to Tautz and Pfeifle (1989). Digoxigenin (DIG) labeled antisense RNA probes were generated with the DIG RNA Labeling Kit (SigmaAldrich) from linearized $N p$, tpr and lint cDNAs according to suppliers guidelines. A hydrolytic digest was subsequently performed by adding $15 \mu \mathrm{l}$ Rase-free water and $25 \mathrm{\mu l}$ carbonate buffer followed by incubation at $70^{\circ} \mathrm{C}$ for $15 \mathrm{~min}$. For RNA precipitation, $50 \mu \mathrm{l} 0.25 \mathrm{M} \mathrm{NaAc}, 10 \mu \mathrm{l} 4 \mathrm{M} \mathrm{LiCl}, 10 \mu \mathrm{l}$ tRNA (20 mg/ml, SigmaAldrich) and $300 \mathrm{\mu l}$ ethanol were added and the mix was incubated at $-20^{\circ} \mathrm{C}$ over night. The precipitated RNA was then spun down for $15 \mathrm{~min}$ at $15000 \mathrm{rpm}$ and 4 ${ }^{\circ} \mathrm{C}$ followed by washing of the RNA pellet with $500 \mu \mathrm{ll} 70 \%$ ethanol. Pelleted RNA probes were then air-dried, dissolved in 150 $\mathrm{\mu l}$ Hybridization solution and stored at $-20^{\circ} \mathrm{C}$.

Embryos for RNA in situ hybridizations were collected and dechorionated as described in chapters 4.3.1 - 4.3.2. Dechorionated embryos were transferred into scintillation tubes containing $1 \mathrm{ml}$ RNA-fix solution and $6 \mathrm{ml}$ heptane and agitated at $300 \mathrm{rpm}$ for $20 \mathrm{~min}$. The lower fixative phase was then removed and $8 \mathrm{ml}$ methanol were added followed by $3 \mathrm{~min}$ shaking at $500 \mathrm{rpm}$ to detach the vitteline membranes. After removal of the supernatant, embryos were washed with methanol repeatedly and stored in methanol at $-20{ }^{\circ} \mathrm{C}$. Fixed embryos were transferred to fresh cups and washed with methanol:PBT 1:1 (v/v) followed by washing with PBT twice. Subsequently, the embryos were incubated in RNA-fix solution:PBT 1:1 (v/v) for $10 \mathrm{~min}$ and then washed four times with PBT. Proteinase-K solution diluted 1:100 in PBT was added to the embryos for $3 \mathrm{~min}$ followed by washing with PBT twice. The embryos were then again fixed with RNA-fix solution:PBT 1:1 (v/v) for 20 min followed by four washing steps with PBT. A 1:1 (v/v) mix of Pre-hybridization solution:PBT was then applied to the embryos followed by washing with Pre-hybridization solution and finally, incubation in Hybridization solution for 1 hour at $70{ }^{\circ} \mathrm{C}$ in a water bath. After removal of the supernatant, $3 \mathrm{\mu l}$ of DIG-labeled antisense-RNA-probe mixed with $30 \mathrm{\mu l}$ 
Hybridization solution was added to the embryos and in situ RNA-hybridization was performed over night at $70^{\circ} \mathrm{C}$ in a water bath. The next day, embryos were washed three times with pre-warmed Hybridization solution for $15 \mathrm{~min}$ at $70{ }^{\circ} \mathrm{C}$ in a water bath. Embryos were then washed with PBT six times. For detection of the DIG-labeled RNA-probes, embryos were then incubated with anti-DIG-AP antibodies diluted in PBT for 2 hours followed by six washing steps with PBT. Embryos were then transferred to a glass dish and incubated in NBT/BCIP solution (Thermo Fisher Scientific) until appropriate staining was visible. Embryos were then mounted on microscope slides in PBS or Prolong mounting medium (Thermo Fisher Scientific), covered with a cover slip and analyzed by bright field light microscopy with an Axiophot microscope (Zeiss). All incubation steps were performed on a rotating wheel at room temperature if not indicated otherwise.

\subsubsection{Cuticle preparations}

Cuticle preparations were performed according to Nüsslein-Volhard et al. (1984). Embryos were collected and dechorionated as described in chapters 4.3.1 - 4.3.2 and then transferred to a cup containing $300 \mu \mathrm{l}$ heptane. For devitellinization, $1 \mathrm{ml}$ methanol was added and the cups were vortexed for 30 sec. The embryos were then repeatedly washed with methanol and rehydrated with PBT. Finally, embryos were mounted in Hoyer's medium and incubated overnight at $65{ }^{\circ} \mathrm{C}$. Images were acquired by dark field light microscopy with an Axiophot microscope (Zeiss).

\subsubsection{Transmission electron microscopy of embryonic trachea}

Stage 17 embryos were collected and dechorionated as described in chapters 4.3.14.3.2, transferred to a $150 \mu \mathrm{m}$ specimen planchette (Engineering Office $\mathrm{M}$. Wohlwend $\mathrm{GmbH}$ ), and frozen with a Leica HBM 100 high-pressure freezer (Leica Microsystems). Vitrified samples were embedded with an Automatic Freeze Substitution Unit (AFS; Leica Microsystems) at $-90{ }^{\circ} \mathrm{C}$ in a solution containing anhydrous acetone, $0.1 \%$ tannic acid, and $0.5 \%$ glutaraldehyde for 72 hours and in anhydrous acetone, $2 \% \mathrm{OsO}$, and $0.5 \%$ glutaraldehyde for additional 8 hours. Samples were then incubated at $-20{ }^{\circ} \mathrm{C}$ for 18 hours followed by warm-up to $4{ }^{\circ} \mathrm{C}$ and subsequent washing with anhydrous acetone. Embedding in Agar 100 (Epon 
812 equivalent) was performed at room temperature and polymerization at $60{ }^{\circ} \mathrm{C}$ for 24 hours. Counterstaining of ultrathin sections was done with $1 \%$ uranylacetate in methanol. Images were taken in a CM120 electron microscope (Philips) using a TemCam F416 CMOS camera (TVIPS).

\subsubsection{Preparation of embryo lysates}

Embryos at the desired developmental stage were collected and dechorionated as described in chapters 4.3.1 - 4.3.2. 100 embryos were then collected by using a Stemi SV 11 stereomicroscope (Zeiss) and transferred to eppendorf cups on ice containing $50 \mu \mathrm{l}$ PBT. If necessary, embryos of certain mutant genotypes were selected by presence or absence of the $d f d$-eYFP balancer chromosome marker. Embryos were then manually squashed with a dissecting needle followed by pulsing with ultrasound in a Bioraptor sonication device (Diagenode) for $30 \mathrm{sec}$. Subsequently, $25 \mu \mathrm{l} 4 \mathrm{x}$ SDS sample buffer were added and the embryo lysate was incubated at $96{ }^{\circ} \mathrm{C}$ for $9 \mathrm{~min}$. After centrifugation at $11000 \mathrm{rpm}$ for 20 minutes, embryo lysate samples were transferred to fresh cups and stored at $-20^{\circ} \mathrm{C}$.

\subsubsection{Quantification and analysis of tracheal cuticle phenotypes}

For quantification of embryos with wild-type-like organization of tracheal cuticle chitin strands and analyses of chitin strands, stage 17 embryos were stained with fluorophore-labeld CBP (NEB) as described in chapter 4.3.6 and imaged by confocal microscopy. For each genotype, 40 embryos were analyzed and defined as embryos with "wild-type-like chitin strands organization" if the chitin strands showed a parallel organization, perpendicular to the longitudinal dorsal trunk axis. Measurements for "diameter of chitin strands" and "chitin strands per $10 \mu \mathrm{m}$ dorsal trunk length" were performed in 10 embryos for each analyzed genotype. For each embryo, two measurements of "chitin strands per $10 \mu \mathrm{m}$ dorsal trunk length" and twenty "diameter of chitin strands" measurements were done using Fiji software (Schindelin et al. 2012). Measurements were performed in dorsal trunk sections of abdominal segments A5-A7. For "chitin strands per $10 \mu \mathrm{m}$ dorsal trunk length" measurements, the chitin strands that were present in a $10 \mu \mathrm{m}$ section of longitudinal dorsal trunk length were counted. Diameters of chitin strands were 
measured by plotting intensity profiles of the longitudinal dorsal trunk axis from images with focal planes of the chitin strands. Lengths of intensity peaks were then measured to determine the diameter of chitin strands. See Appendix 7.2 for measurement data.

\subsection{Cell culture-based experiments}

D. melanogaster S2R+ cells (DGRC) and Kc167 cells (DGRC) were kept in flasks containing Schneider's Drosophilamedium (Thermo Fisher Scientific) supplemented with $1 \%$ Penicillin/Streptomycin (Thermo Fisher Scientific) and 10 $\%$ FBS (Sigma-Aldrich) at $25{ }^{\circ} \mathrm{C}$. Handling of cells was performed in the sterile environment of a clean bench.

\subsubsection{Transfection of cultured cells}

Confluent cells were detached, diluted 1:6, and transferred either to $10 \mathrm{~cm}$ diameter petri dishes (Greiner Bio One; $7 \mathrm{ml}$ per dish), to 6-well plates (Greiner Bio One; $3 \mathrm{ml}$ per well), or to glass bottom micro-well dishes (MatTek; $6 \mathrm{ml}$ per dish), approximately 24 hours before transfection. The UAS/Gal4 system was used for protein over-expression in cultured cells. Cells in 6-well plates and glass bottom micro-well dishes were transfected with $500 \mathrm{ng}$ actin5C-Gal4 vector and $500 \mathrm{ng}$ pUAST-responder vector ( $1 \mu \mathrm{g}$ total DNA), while cells in $10 \mathrm{~cm}$ diameter petri dishes were transfected with twice the amount of each vector $(2 \mu \mathrm{g}$ total DNA). The Effectene transfection reagent (Qiagen) was used for cell transfection according to suppliers guidelines with minor alterations. For transfections of cells in 6-well plates and glass bottom micro-well dishes, vector DNA was mixed with $190 \mu \mathrm{l}$ Effectene EC buffer (Qiagen) followed by adding $8 \mu$ l Effectene Enhancer (Qiagen), vortexing and incubation for $5 \mathrm{~min}$. Subsequently, $20 \mu \mathrm{l}$ Effectene were added and the mix was vortexed for $10 \mathrm{sec}$ followed by incubation for $15 \mathrm{~min}$. The transfection mix was then carefully trickled onto the cells with a pipette. Incubation steps were performed at room temperature. For transfections of cells in $10 \mathrm{~cm}$ diameter petri dishes with $2 \mu \mathrm{g}$ DNA, volumes of the transfection mix components were doubled. 


\subsubsection{Live-imaging of cultured cells}

Cells in glass bottom micro-well dishes were incubated at $25{ }^{\circ} \mathrm{C}$ for approximately 48 hours after transfection followed by imaging with a confocal LSM.

\subsubsection{Antibody stainings of cultured cells}

Cells in glass bottom micro-well dishes were incubated at $25^{\circ} \mathrm{C}$ for approximately 48 hours after transfection and then washed with PBS twice and fixed by adding formaldehyde solution and incubation for $15 \mathrm{~min}$. The cells were then washed twice with PBS and incubated in PBTX for 150 sec for permeabilization. Subsequently, cells were washed three times with PBS followed by saturation of unspecific immunoglobulin binding sites by incubation in blocking solution for $30 \mathrm{~min}$. Then, primary antibodies diluted in blocking solution were added to the cells and incubated for 2 hours. The cells were then again washed three times with PBS followed by incubation with secondary antibodies diluted in blocking solution for 1 hour. Finally, the cells were washed twice with PBS followed by mounting in Prolong mounting medium with DAPI (Thermo Fisher Scientific) that was added to the glass bottom micro-well dishes and mounted cells were covered with a cover slip. Images were acquired with a confocal LSM. All incubation and washing steps were performed at room temperature.

\subsubsection{Preparation of cell lysates and supernatant precipitates}

The medium of cells in 6-well plates was exchanged with Schneider's Drosophila medium supplemented with $1 \%$ Penicillin/Streptomycin (FBS-free medium) approximately 24 hours after transfection. After approximately 48 hours more (72 hours after transfection), the supernatants were collected in cups and the cells were detached by pipetting up and down in $1 \mathrm{ml}$ PBS and collected in cups. The cell samples and supernatant samples were then centrifuged at $900 \mathrm{rpm}$ and $4{ }^{\circ} \mathrm{C}$ for 5 $\min .800 \mu \mathrm{l}$ of supernatant samples were transferred to fresh cups which contained $200 \mu \mathrm{l}$ TCA solution and incubated over night at $4{ }^{\circ} \mathrm{C}$. The cells were washed a second time with PBS and again centrifuged at $900 \mathrm{rpm}$ and $4{ }^{\circ} \mathrm{C}$ for $5 \mathrm{~min}$. Subsequently, PBS was removed from the pelleted cells and they were resuspended in $100 \mu \mathrm{l}$ PBS followed by addition of $35 \mu \mathrm{l} 4 \mathrm{x}$ SDS sample buffer and incubation at 
$96{ }^{\circ} \mathrm{C}$ for $9 \mathrm{~min}$. Cell lysate samples were stored at $-20{ }^{\circ} \mathrm{C}$.The next day, supernatant precipitate samples were centrifuged at $15000 \mathrm{rpm}$ and $4{ }^{\circ} \mathrm{C}$ for 20 min. The pellets of precipitated proteins were washed twice with acetone on ice and then air-dried. Finally, pellets were resuspended in $100 \mu \mathrm{l} 1 \mathrm{x}$ SDS sample buffer and incubated at $96^{\circ} \mathrm{C}$ for $9 \mathrm{~min}$. Supernatant precipitate samples were stored at $-20{ }^{\circ} \mathrm{C}$.

\subsubsection{Purification of proteins from cultured cells and cell culture supernatants}

Flag::Pio and Flag::Pio ${ }^{\text {R196A }}$ expressing cells were harvested from $10 \mathrm{~cm}$ diameter petri dishes 72 hours after transfection by discarding the medium, detaching the cells by pipetting up and down in $1 \mathrm{ml}$ PBS and transfer of the detached cells to a fresh cup. Cells were then centrifuged at $900 \mathrm{rpm}$ and $4{ }^{\circ} \mathrm{C}$ for 5 min followed by washing with PBS and another centrifugation step at $900 \mathrm{rpm}$ and $4{ }^{\circ} \mathrm{C}$ for $5 \mathrm{~min}$. Supernatant PBS was then discarded and the cells were lysed by pipetting up and down in $200 \mu$ lysis buffer for $30 \mathrm{~min}$ followed by centrifugation at $15000 \mathrm{rpm}$ and $4{ }^{\circ} \mathrm{C}$ for $12 \mathrm{~min}$. Flag::Pio and Flag::Pio ${ }^{\text {R196A }}$ were subsequently purified from the cell lysates using Anti-Flag Magnetic Agarose (Thermo Fisher Scientific) according to the suppliers protocol. Elution of the proteins from the Anti-Flag Magnetic Agarose was done by adding $50 \mu \mathrm{l}$ 0,2 M glycine (pH 2,5) for $30 \mathrm{sec}$ and transferring the eluted proteins to a fresh cup that contained $8 \mu l 1 \mathrm{M}$ Tris $(\mathrm{pH}$ 10,4) for neutralization.

For purification of proteases from cell culture supernatants, the medium of cells in $10 \mathrm{~cm}$ diameter petri dishes was exchanged with Schneider's Drosophila medium supplemented with $1 \%$ Penicillin/Streptomycin (FBS-free medium) approximately 24 hours after transfection. Supernatants of Tpr::Flag and $\mathrm{Tpr}^{\mathrm{ZL}}::$ Flag expressing cells were harvested 72 hours after transfection and centrifuged at $900 \mathrm{rpm}$ and 4 ${ }^{\circ} \mathrm{C}$ for 5min. Tpr::Flag and $\mathrm{Tpr}^{\mathrm{ZL}}:$ :Flag were subsequently purified from the supernatants by using Anti-Flag Magnetic Agarose (Thermo Fisher Scientific) according to the suppliers protocol. Elution of the proteins from the Anti-Flag Magnetic Agarose was done by adding $50 \mu \mathrm{l}$ 0,2 $\mathrm{M}$ glycine ( $\mathrm{pH}$ 2,5) for 30 sec and transferring the eluted proteins to a fresh cup that contained $8 \mu \mathrm{l} 1 \mathrm{M}$ Tris $(\mathrm{pH}$ 10,4) for neutralization. 
Supernatants of cells that expressed Strep-tagged $\mathrm{Np}, \mathrm{Np}^{\mathrm{S990A}}$, matriptase, or matriptase $^{\mathrm{S} 805 \mathrm{~A}}$ were harvested 72 hours after transfection and centrifuged at 900 rpm and $4{ }^{\circ} \mathrm{C}$ for $5 \mathrm{~min}$. Strep-tagged proteins were then purified from the supernatants by using MagStrep type3 XT Magnetic Beads (IBA) according to the suppliers protocol. Proteins were eluted by adding $75 \mu \mathrm{l}$ Buffer BXT (IBA) and incubation for $10 \mathrm{~min}$ on ice with frequent vortexing. Eluted proteins were then transferred to a fresh cups.

All steps of protein purification were performed at $4{ }^{\circ} \mathrm{C}$ or on ice. After purification, protein concentrations were determined with a NanoDrop ND-1000 spectrophotometer (VWR) according to the manufacturers instructions. For storage, purified protein samples were shock-frozen in liquid nitrogen and stored at $-80^{\circ} \mathrm{C}$.

\subsection{Biochemical assays}

Purified proteins were thawed on ice. $1 \mu \mathrm{g}$ of potential protease substrates (Flag::Pio, Flag::Pio ${ }^{\text {R196A }}$, Tpr::Flag or Tpr ${ }^{\mathrm{ZL}:: F l a g)}$ were mixed with $1 \mu \mathrm{g}$ of respective protease (Np::Strep, Np ${ }^{\text {S990A }:: S t r e p, ~ m a t r i p t a s e:: S t r e p ~ o r ~}$ matriptase ${ }^{\mathrm{S} 805 \mathrm{~A}}::$ Strep) in $15 \mu \mathrm{l}$ Protease reaction buffer. As controls, assays that contained only the potential substrate or only the respective protease were set up. The assays were incubated at $22^{\circ} \mathrm{C}$ over night. Subsequently, $10 \mu \mathrm{l}$ x SDS sample buffer were added followed by incubation at $96{ }^{\circ} \mathrm{C}$ for $9 \mathrm{~min}$. Samples were stored at $-20{ }^{\circ} \mathrm{C}$.

\subsection{SDS-PAGE and western blotting}

Denatured and reduced samples were loaded onto 4-20\% gradient Mini-Protean TGX Precast Protein Gels (BioRad) together with PageRuler prestained protein ladder (Thermo Scientific). Gels were run in a MiniProtean chamber (BioRad) that was filled with SDS running buffer and connected to a PowerPac Basic power supply (BioRad) for $40 \mathrm{~min}$ at $170 \mathrm{~V}$. The gels were then equilibrated in transfer 
buffer and packed into a western blotting sandwich. Blotting sandwiches were set up with outermost sponges, followed by layers of blotting filter paper (Thermo Fisher Scientific) and the gel and transfer membrane in the center. PVDF transfer membrane with $0,2 \mu \mathrm{m}$ pore size (Thermo Fisher Scientific) was used for analysis of embryos lysates. In all other case, Amersham Hybond-ECL membrane with 0,2 $\mu \mathrm{m}$ pore size (GE Healthcare) was used. Protein transfer to the membrane was performed on ice in a MiniProtean chamber (BioRad) that was filled with transfer buffer at $300 \mathrm{~mA}$ for $90 \mathrm{~min}$.

\subsubsection{Antibody stainings of western blots}

After western blotting, the membranes were washed four times with PBT for 15 min per wash. Then, Casein Blocking Buffer (10x; Sigma-Aldrich) diluted 1:10 in PBT was applied to the membrane and incubated for 1 hour. Blocking buffer was then removed and primary antibodies diluted in 1:10 Casein Blocking Buffer:PBT were added and the membranes were incubated at $4{ }^{\circ} \mathrm{C}$ over night. The next day, membranes were again washed four times with PBT for 15 min per wash, followed by incubation with secondary antibodies diluted in 1:10 Casein Blocking Buffer:PBT for 2 hours. Subsequently, membranes were washed with PBT 4 times (15 min per wash) and WesternBright Sirius chemiluminescent detection reagent (Biozym) was applied to the membranes. Chemiluminescent signals were then detected in an Intelligent Dark BoxII (Fujifilm) with the LAS-1000 software (Fujifilm). All incubation steps were performed on a horizontal shaker at $20 \mathrm{rpm}$ and at room temperature if not indicated otherwise.

\subsection{Light microscopy and image acquisition}

Stemi SV 11 or Stemi 2000 stereomicroscopes (Zeiss) connected to an external limelight source KL1500 LCD (Schott) or an internal limelight source were used for observation of flies and larvae as well as for collection of specific amounts of embryos. Bright field and dark field light microscopy was done with an Axiophot microscope (Zeiss) and images were acquired with an AxioCam HRc and the Zen acquisition software (Zeiss). Analyses of fluorescent stainings of embryos and 
cultured cells as well as in vivo imaging of fluorphore-labeled protein expressing embryos and cultured cells were performed with a LSM780 confocal microscope (Zeiss) and the Zen acquisition software (Zeiss). A LD LCI Plan-Apochromat 25×/0.8 Imm Corr DIC M27, a Plan-Apochromat 40×/1.4 Oil DIC M27 oil immersion or a $63 \times / 1.3$ Imm Corr DIC M27 LCI Plan-Neofluar (water) objective were used for confocal LSM imaging.

\subsection{DNA purification, amplification and molecular cloning}

\subsubsection{Purification of Drosophila melanogaster genomic DNA}

The genomic DNA of $D$. melanogaster strains with lethal mutations was purified from homozygous mutant embryos. The embryos were collected and dechorionated as described in chapters 4.3.1 - 4.3.2. Then, $30-40$ homozygous mutant embryos were selected by absence of the $\mathrm{CyO}$, dfd-eYFP balancer chromosome and transferred to a cup with $180 \mu \mathrm{l}$ PBS on ice. For purification of genomic DNA from adult flies, 20-30 flies were collected in a cup and killed by freezing at $-20{ }^{\circ} \mathrm{C}$ for 15 min and $180 \mu \mathrm{l}$ PBS were subsequently added to the flies. Collected flies or embryos were then homogenized with a pistil followed by purification of genomic DNA with the DNeasy Blood \& Tissue Kit (Qiagen) according to the manufacturers instructions for isolation of total DNA from cultured animal cells.

\subsubsection{Isolation of plasmids from bacterial cultures}

Bacterial liquid cultures were grown in LB medium supplemented with appropriate antibiotics and inoculated with a single colony by using a sterile pipett tip. Liquid bacterial cultures were grown over night at $37{ }^{\circ} \mathrm{C}$ and $180 \mathrm{rpm}$. Smallscale plasmid isolations were done from $3 \mathrm{ml}$ bacterial cultures with the NucleoSpin Plasmid Kit (Macherey-Nagel) according to the manufacturers guidelines. Plasmids that were used for mutagenesis and transgenesis of $D$. melanogaster or were used for cell culture experiments were isolated from $50 \mathrm{ml}$ 
bacterial cultures using the Plasmid Plus Midi Kit (Qiagen) according to the manufacturers guidelines.

\subsubsection{Measurement of DNA concentrations}

Concentrations of purified genomic DNA, plasmid DNA or DNA fragments was determined by measurements of the absorption at $260 \mathrm{~nm}$ wavelength with a NanoDrop ND-1000 spectrophotometer (VWR) according to the manufacturers guidelines.

\subsubsection{Amplification of DNA by polymerase chain reaction}

DNA fragments for sequencing or molecular cloning were amplified by PCR using Phusion-HF DNA polymerase (NEB) or Q5-HF DNA polymerase (NEB) according to the suppliers protocols. The PCR reactions contained primers (Table 6) at a concentration of $0,5 \mu \mathrm{M}$ per primer, dNTPs (Thermo Fisher Scientific) at a concentration of $0,2 \mathrm{mM}$ of each dNTP and $100-500 \mathrm{ng}$ genomic template DNA or 0,5-10 ng plasmid template DNA. Annealing temperatures of primers were calculated by the nearest neighbors method. PCR reactions were run on a MyCycler Thermal Cycler (Biorad) according to suppliers guidelines and calculated annealing temperatures. Primers were used to add endonuclease restriction sides, overlapping sequences for Gibson assembly, or changes in the DNA sequence to the amplified DNA fragments (see Table 6 for primers and their features).

Analytical colony PCRs were performed with the HotStarTaq Master Mix Kit (Qiagen) to determine whether plasmids in bacterial colonies contained certain inserts or deletions. A colony PCR reaction contained $5 \mu$ l HotStarTaq Master Mix, $1 \mu \mathrm{l}$ of each primer $(10 \mu \mathrm{M}$; final concentration $1 \mu \mathrm{M})$ and $3 \mu \mathrm{l}$ sterile water. A single bacterial colony was dipped into a colony PCR reaction with a sterile pipett tip and subsequently used for inoculation of $3 \mathrm{ml} \mathrm{LB}$ medium. Colony PCR reactions were run on a MyCycler Thermal Cycler (Biorad) according to suppliers guidelines and subsequently analyzed by agarose gel electrophoresis. 


\subsubsection{Endonuclease restriction digests of DNA}

All restriction endonucleases were supplied by NEB. Reactions were set up according to protocols for the respective enzymes, with total DNA substrates of 0,5 $-3 \mu \mathrm{g}$ per reaction. Reactions were stopped by heat inactivation, freezing at $-20{ }^{\circ} \mathrm{C}$ or by directly applying DNA gel loading dye (Thermo Fisher Scientific) to the reactions followed by agarose gel electrophoresis.

\subsubsection{Agarose gel electrophoresis of DNA}

When necessary, DNA fragments were separated by agarose gel electrophoresis according to their size. $1 \mathrm{~g}$ agarose was dissolved in $100 \mathrm{ml} \mathrm{1x}$ TAE buffer by boiling for $2 \mathrm{~min}$. Then, $8 \mu \mathrm{l}$ ethidium bromide solution or $10 \mu \mathrm{l}$ Midori Green (Nippon Genetics Europe) were added and the gel was poured into a gel tray for hardening. DNA samples were supplemented with DNA gel loading dye (Thermo Fisher Scientific) and loaded onto the hardened gel. The GeneRuler DNA ladder (Thermo Fisher Scientific) was also applied to the gel for size reference of the DNA fragments. Agarose gel electrophoresis was then performed in a chamber containing $1 \mathrm{x}$ TAE running buffer at $100 \mathrm{~V}$. A UV Solo TS transilluminator (Biometra) was used for visualization of DNA fragments and documentation.

\subsubsection{Purification of DNA fragments}

DNA fragments that were amplified by PCR or generated by endonuclease restriction digests were purified with the NucleoSpin Gel and PCR Clean-up Kit (Macherey-Nagel) according to suppliers guidelines. Purifications were either performed directly after PCRs or restriction digests, or after agarose gel electrophoresis. In this case, the piece of gel that contained the desired DNA fragment was cut out by using a surgical blade. 


\subsubsection{Ligation of DNA fragments}

Ligation of DNA fragments into vector backbones was either done with the Quick Ligase kit (NEB), the T4-Ligase (Thermo Fisher Scientific) or by Gibson assembly with the NEBuilder HiFi DNA assembly Master Mix (NEB).

For ligation of sgRNA target sequences into $p B F_{V}-U 6.2$ or $p B F_{V}-U 6.2 B$ vectors, DNA oligos of the target sequences (Table 6) were first annealed in a reaction that contained the sense- and antisense oligos at a concentration of $10 \mu \mathrm{M}$ each in $1 \mathrm{x}$ T4-Ligase buffer (Thermo Fisher Scientific). This reaction was heated to $95{ }^{\circ} \mathrm{C}$ and then ramped down to $25^{\circ} \mathrm{C}$ at a rate of $-0.1{ }^{\circ} \mathrm{C} / \mathrm{sec}$ in a MyCycler Thermal Cycler (Biorad). The annealed target sequence was diluted 1:200 in sterile water of which $1 \mu \mathrm{l}$ was mixed with $50 \mathrm{ng} p B F_{V-} U 6.2$ or $p B F_{V}-U 6.2 B$ vector that was digested with BbsI restriction enzyme (NEB) and $1 \mu \mathrm{l}$ T4-Ligase in $10 \mu \mathrm{l} 1 \mathrm{x}$ T4-Ligase buffer. This reaction was incubated at room temperature for 1 hours and subsequently used for transformation of competent bacteria.

Ligations of inserts into linearized vectors that were both generated by endonuclease restriction digests were performed with the Quick Ligase kit (NEB) according to suppliers guidelines. Vectors and inserts were used in a molar ration of $1: 3$ in these reactions. For Gibson assembly of inserts and vector backbones, the used DNA fragments were generated with homologous overhangs by PCR (see Table 6 for primers). The Gibson assembly reactions were carried out with the NEBuilder HiFi DNA assembly Master Mix (NEB) according to suppliers guidelines.

\subsubsection{Transformation of chemically competent bacteria}

Ligated or assembled vectors were amplified and selected by transformation of competent E. coli bacteria. $50 \mu \mathrm{l}$ chemically competent TOP10 or DH5a E. coli cells (Thermo Fisher Scientific) were thawed on ice and $10 \mu \mathrm{l}$ of ligation- or Gibson assembly reactions were added, followed by incubation for $20 \mathrm{~min}$ on ice. The cups with competent bacteria were then transferred to a water bath and heat shocked at $42{ }^{\circ} \mathrm{C}$ for $30 \mathrm{sec}$. Then, the cells were incubated on ice for $2 \mathrm{~min}$ followed by addition of $900 \mu \mathrm{l} \mathrm{LB}$ medium and incubation at $37^{\circ} \mathrm{C}$ and $180 \mathrm{rpm}$ for $40 \mathrm{~min}$. 
The bacteria suspensions were then spread out on LB agar plates that contained the appropriate antibiotics and incubated at $37^{\circ} \mathrm{C}$ over night.

\subsubsection{DNA sequencing}

Vectors and PCR amplicons were sequenced by MWG Eurofins genomics or Microsynth Seqlab. Primers that are listed in Table 6 were used for sequencing or primers were chosen from the available standard sequencing primers of the companies.

\subsection{Software, algorithms and data repositories}

Data and images were analyzed and arranged into figures by using Fiji software (Schindelin et al. 2012), Adobe Illustrator (Adobe Systems) and Adobe Photoshop (Adobe Systems). DNA sequences were analyzed with the DNASTAR software suite (DNAStar). Databases of the National Institutes of Health (NCBI; www.ncbi.nlm.nih.gov/pubmed/) and Flybase (Gramates et al. 2017; www.flybase.org) were used for research. The BLASTP algorithm (Altschul et al. 1997) was used for comparative analysis of protein sequences from different species and the Clustal omega algorithm (Sievers et al. 2011) was used for alignment of amino acid sequences. The NetOGlyc 4.0 algorithm (Steentoft et al. 2013), the TMHMM 2.0 algorithm (Krogh et al. 2001) and the SignalP 4.1 algorithm (Nielsen 2017) were used for prediction of glycosylation sites, transmembrane helices and signal peptides in protein sequences. 


\section{List of Abbreviations}

\begin{tabular}{|c|c|c|c|}
\hline${ }^{\circ} \mathrm{C}$ & degree centigrade & Kune & Kune-kune \\
\hline$A b d-B$ & $A b d o m i n a l-B$ & $\mathrm{~L}$ & liter \\
\hline aECM & apical extracellular matrix & $\mathrm{LB}$ & lysogeny broth \\
\hline $\mathrm{AP}$ & alkaline phosphatase & $\mathrm{LC}$ & liquid clearance \\
\hline BLAST & Basic Local Alignment Search Tool & Lint & Lumens interrupted \\
\hline $\mathrm{Bnl}$ & Branchless & LSM & laser-scanning-microscope \\
\hline bp & base pair & $\mathrm{m}$ & meter \\
\hline Btl & Breathless & M & molar \\
\hline Cas9 & CRISPR associated protein 9 & $\mathrm{MDa}$ & megadalton \\
\hline CBP & chitin binding probe & Mega & Megatrachea \\
\hline cDNA & complementary DNA & $\min$ & minute \\
\hline $\mathrm{Crb}$ & Crumbs & $\mathrm{ml}$ & milliliter \\
\hline CRISPR & $\begin{array}{l}\text { clustered regularly interspaced } \\
\text { short palindromic repeats }\end{array}$ & $\mathrm{mM}$ & millimolar \\
\hline DE-cad & DE-cadherin & $\mathrm{Np}$ & Notopleural \\
\hline Dfd & Deformed & Obst-A & Obstructor-A \\
\hline DIG & Digoxygenin & ORF & open reading frame \\
\hline DNA & deoxyribonucleic acid & PAGE & polyacrylamid gel electrophoresis \\
\hline Dpp & Decapentaplegic & PAM & protospacer adjacent motif \\
\hline Dpy & Dumpy & PAR-2 & proteinase-activated receptor 2 \\
\hline $\mathrm{ECM}$ & extracellular matrix & PCR & polymerase chain reaction \\
\hline $\mathrm{ENaC}$ & epithelial sodium channel & Pio & Piopio \\
\hline EpCam & epithelial cell adhesion molecule & RNA & ribonucleic acid \\
\hline $\operatorname{Exp}$ & Expansion & RNAi & RNA interference \\
\hline Fas3 & Fasciclin 3 & $\mathrm{rpm}$ & rounds per minute \\
\hline FGF & fibroblast growth factor & Rtv & Retroactive \\
\hline $\mathrm{g}$ & gram & SDS & sodium dodecyl sulfate \\
\hline GFP & Green Fluorescent Protein & sec & second \\
\hline GPI & glycophosphatidylinositol & Serp & Serpentine \\
\hline HAI & $\begin{array}{l}\text { hepatocyte growth factor activator } \\
\text { inhibitor }\end{array}$ & sgRNA & single guide RNA \\
\hline $\mathrm{kb}$ & kilobase & Shot & Shortstop \\
\hline $\mathrm{kDa}$ & kilodalton & $S T 14$ & suppression of tumorigenicity-14 \\
\hline $\mathrm{Kkv}$ & Krotzkopf verkehrt & TGF-B & transforming growth factor beta \\
\hline Knk & Knickkopf & TGFBR3 & TGF-B receptor type III \\
\hline
\end{tabular}




$\begin{array}{ll}\text { Tpr } & \text { Tracheal-prostasin } \\ \text { Trh } & \text { Trachealess } \\ \text { TTSP } & \text { type II transmembrane serine protease } \\ \text { Tyn } & \text { Trynity } \\ \text { UAS } & \text { upstream activating sequence } \\ \text { Uif } & \text { Uninflatable } \\ \text { Verm } & \text { Vermiform } \\ { }^{\prime} & \text { white } \\ \text { YFP } & \text { Yellow Fluorescent Protein } \\ \text { ZPD } & \text { Zona pellucida domain } \\ \mu & \text { micro }\end{array}$




\section{References}

Affolter M, Caussinus E. Tracheal branching morphogenesis in Drosophila: new insights into cell behaviour and organ architecture. Development. 2008; 135(12): 2055-64. doi: 10.1242/dev.014498.

Aggarwal S, Dabla PK, Arora S. Prostasin: An Epithelial Sodium Channel Regulator. J Biomark. 2013; 2013: 179864. doi: 10.1155/2013/179864.

Alef T, Torres S, Hausser I, Metze D, Türsen U, Lestringant GG, Hennies HC. Ichthyosis, follicular atrophoderma, and hypotrichosis caused by mutations in ST14 is associated with impaired profilaggrin processing. J Invest Dermatol. 2009; 129(4): 862-9. doi: 10.1038/jid.2008.311.

Altschul SF, Madden TL, Schäffer AA, Zhang J, Zhang Z, Miller W, Lipman DJ. Gapped BLAST and PSI-BLAST: a new generation of protein database search programs. Nucleic Acids Res. 1997; 25(17): 3389-402.

Andrew DJ, Ewald AJ. Morphogenesis of epithelial tubes: Insights into tube formation, elongation, and elaboration. Dev Biol. 2010; 341(1): 34-55. doi: 10.1016/j.ydbio.2009.09.024.

Antalis TM, Bugge TH, Wu Q. Membrane-anchored serine proteases in health and disease. Prog Mol Biol Transl Sci. 2011; 99: 1-50. doi: 10.1016/B978-0-12385504-6.00001-4.

Armbruster K, Luschnig S. The Drosophila Sec7 domain guanine nucleotide exchange factor protein Gartenzwerg localizes at the cis-Golgi and is essential for epithelial tube expansion. J Cell Sci. 2012; 125(Pt 5): 1318-28. doi: $10.1242 /$ jcs.096263.

Babatz F, Naffin E, Klämbt C. The Drosophila Blood-Brain Barrier Adapts to Cell Growth by Unfolding of Pre-existing Septate Junctions. Dev Cell. 2018; 47(6): 697-710.e3. doi: 10.1016/j.devcel.2018.10.002.

Banerjee S, Sousa AD, Bhat MA. Organization and function of septate junctions: an evolutionary perspective. Cell Biochem Biophys. 2006; 46(1): 65-77.

Beaulieu A, Gravel É, Cloutier A, Marois I, Colombo É, Désilets A, Verreault C, Leduc R, Marsault É, Richter MV. Matriptase proteolytically activates influenza virus and promotes multicycle replication in the human airway epithelium. J Virol. 2013; 87(8): 4237-51. doi: 10.1128/JVI.03005-12. 
Behr M, Wingen C, Wolf C, Schuh R, Hoch M. Wurst is essential for airway clearance and respiratory-tube size control. Nat Cell Biol. 2007; 9(7): 847-53. doi: $10.1038 /$ ncb1611

Behr M, Riedel D, Schuh R. The claudin-like Megatrachea is essential in septate junctions for the epithelial barrier function in Drosophila. Dev Cell. 2003; 5: 611-620.

Bilandzic M, Stenvers KL. Betaglycan: a multifunctional accessory. Mol Cell Endocrinol. 2011; 339(1-2): 180-9. doi: 10.1016/j.mce.2011.04.014.

Bhatt AS, Welm A, Farady CJ, Vásquez M, Wilson K, Craik CS. Coordinate expression and functional profiling identify an extracellular proteolytic signaling pathway. Proc Natl Acad Sci U S A. 2007; 104(14): 5771-6. doi: 10.1073/pnas.0606514104

Bökel C, Prokop A, Brown NH. Papillote and Piopio: Drosophila ZP-domain proteins required for cell adhesion to the apical extracellular matrix and microtubule organization. J Cell Sci. 2005; 118: 633-642; doi: 10.1242/jcs.01619

Bokhove M, Jovine L. Structure of Zona Pellucida Module Proteins. Curr Top Dev Biol. 2018; 130: 413-442. doi: 10.1016/bs.ctdb.2018.02.007.

Bonnans C, Chou J, Werb Z. Remodelling the extracellular matrix in development and disease. Nat Rev Mol Cell Biol. 2014; 15(12): 786-801. doi: $10.1038 / \mathrm{nrm} 3904$.

Boon L, Ugarte-Berzal E, Vandooren J, Opdenakker G. Glycosylation of matrix metalloproteases and tissue inhibitors: present state, challenges and opportunities. Biochem J. 2016; 473(11): 1471-82. doi: 10.1042/BJ20151154.

Brand AH, Perrimon N. Targeted gene expression as a means of altering cell fates and generating dominant phenotypes. Development. 1993; 118: 401-415.

Bridges CB, Skoog EN, Li JC. Genetical and Cytological Studies of a Deficiency (Notopleural) in the Second Chromosome of Drosophila Melanogaster. Genetics. 1936; 21: 788-795.

Brodu V, Casanova J. The RhoGAP crossveinless-c links trachealess and EGFR signaling to cell shape remodeling in Drosophila tracheal invagination. Genes Dev. 2006; 20(13): 1817-28. doi: 10.1101/gad.375706

Brown JB, Boley N, Eisman R, May GE, Stoiber MH, Duff MO, Booth BW, Wen J, Park S, Suzuki AM, Wan KH, Yu C, Zhang D, Carlson JW, Cherbas L, et al. Diversity and dynamics of the Drosophila transcriptome. Nature. 2014; 512(7515): 393-9. doi: 10.1038/nature12962 
Bugge TH, Antalis TM, Wu Q. Type II transmembrane serine proteases. J Biol Chem. 2009; 284: 23177-23181. doi:10.1074/jbc.R109.021006

Bugge TH, List K, Szabo R. Matriptase-dependent cell surface proteolysis in epithelial development and pathogenesis. Front Biosci. 2007; 12: 5060-70.

Campos-Ortega JA, Hartenstein V. Stages of Drosophila Embryogenesis. In: The Embryonic Development of Drosophila melanogaster. 1997; Springer, Berlin, Heidelberg. Doi: 10.1007/978-3-662-22489-2_2

Carney TJ, von der Hardt S, Sonntag C, Amsterdam A, Topczewski J, Hopkins N, Hammerschmidt M. Inactivation of serine protease Matriptase1a by its inhibitor Hai1 is required for epithelial integrity of the zebrafish epidermis. Development. 2007; 134(19): 3461-71. doi: 10.1242/dev.004556

Carattino MD, Mueller GM, Palmer LG, Frindt G, Rued AC, Hughey RP, Kleyman TR. Prostasin interacts with the epithelial $\mathrm{Na}+$ channel and facilitates cleavage of the Y-subunit by a second protease. Am J Physiol Renal Physiol. 2014; 307(9): F1080-7. doi: 10.1152/ajprenal.00157.2014.

Caviglia S, Luschnig S. Tube fusion: making connections in branched tubular networks. Semin Cell Dev Biol.2014; $31: 82-90$. doi: 10.1016/j.semcdb.2014.03.018.

Centanin L, Dekanty A, Romero N, Irisarri M, Gorr TA, Wappner P. Cell autonomy of HIF effects in Drosophila: tracheal cells sense hypoxia and induce terminal branch sprouting. Dev Cell.2008; 14(4):547-58. doi: 10.1016/j.devcel.2008.01.020.

Chanda D, Otoupalova E, Smith SR, Volckaert T, De Langhe SP, Thannickal VJ. Developmental pathways in the pathogenesis of lung fibrosis. Mol Aspects Med. 2018 pii: S0098-2997(18)30071-2. doi: 10.1016/j.mam.2018.08.004.

Chandran RR, Scholl A, Yang Y, Jiang 2. rebuff regulates apical luminal matrix to control tube size in Drosophila trachea. Biol Open. 2018; 7(9). pii: bio036848. doi: 10.1242/bio.036848.

Chang HH, Xu Y, Lai H, Yang X, Tseng CC, Lai YJ, Pan Y, Zhou E, Johnson MD, Wang JK, Lin CY. Differential subcellular localization renders HAI-2 a matriptase inhibitor in breast cancer cells but not in mammary epithelial cells. PLoS One. 2015; 10(3): e0120489. doi: 10.1371/journal.pone.0120489.

Cheifetz S, Andres JL, Massagué J. The transforming growth factor-beta receptor type III is a membrane proteoglycan. Domain structure of the receptor. J Biol Chem. 1988; 263(32): 16984-91.

Chen CK, Kühnlein RP, Eulenberg KG, Vincent S, Affolter M, Schuh R. The transcription factors KNIRPS and KNIRPS RELATED control cell migration 
and branch morphogenesis during Drosophila tracheal development. Development. 1998; 125(24): 4959-68.

Chen YW, Wang JK, Chou FP, Chen CY, Rorke EA, Chen LM, Chai KX, Eckert RL, Johnson MD, Lin CY. Regulation of the matriptase-prostasin cell surface proteolytic cascade by hepatocyte growth factor activator inhibitor-1 during epidermal differentiation. J Biol Chem. 2010; 285(41): 31755-62. doi: 10.1074/jbc.M110.150367.

Dong B, Hannezo E, Hayashi S. Balance between apical membrane growth and luminal matrix resistance determines epithelial tubule shape. Cell Rep. 2014; 7: 941-950. doi:10.1016/j.celrep.2014.03.066

Dong B, Hayashi S. Shaping of biological tubes by mechanical interaction of cell and extracellular matrix. Curr Opin Genet Dev. 2015; 32: 129-134. doi:10.1016/j.gde.2015.02.009

Drees L, Königsmann T, Jaspers MHJ, Pflanz R, Riedel D, Schuh R. Conserved function of the matriptase-prostasin proteolytic cascade during epithelial morphogenesis. PLoS Genet. $2019 ; 15(1)$ : e 1007882 . doi: 10.1371/journal.pgen.1007882.

Elderbroom JL, Huang JJ, Gatza CE, Chen J, How T, Starr M, Nixon AB, Blobe GC. Ectodomain shedding of TBRIII is required for TBRIII-mediated suppression of TGF- $B$ signaling and breast cancer migration and invasion. Mol Biol Cell. 2014; 25(16): 2320-32. doi: 10.1091/mbc.E13-09-0524.

Elias N, O'Brodovich H. Clearance of Fluid From Airspaces of Newborns and Infants. NeoRev. 2006 ; 7(2): e88-e94. doi: 10.1542/neo.7-2-e88.

Fagerberg L, Hallström BM, Oksvold P, Kampf C, Djureinovic D, Odeberg J, Habuka M, Tahmasebpoor S, Danielsson A, Edlund K, Asplund A, Sjöstedt E, Lundberg E, Szigyarto CA, Skogs M, Takanen JO et al. Analysis of the human tissue-specific expression by genome-wide integration of transcriptomics and antibody-based proteomics. Mol Cell Proteomics. 2014; 13(2): 397-406. doi: 10.1074/mcp.M113.035600.

Fernandes I, Chanut-Delalande H, Ferrer P, Latapie Y, Waltzer L, Affolter M, et al. Zona pellucida domain proteins remodel the apical compartment for localized cell shape changes. Dev Cell. 2010; 18: 64-76. doi:10.1016/j.devcel.2009.11.009

Förster TD, Woods HA. Mechanisms of tracheal filling in insects. Biol Rev Camb Philos Soc. 2013; 88(1): 1-14. doi: 10.1111/j.1469-185X.2012.00233.x.

Förster D, Luschnig S. Src42A-dependent polarized cell shape changes mediate epithelial tube elongation in Drosophila. Nat Cell Biol. 2012; 14(5): 526-34. doi: $10.1038 /$ ncb2456. 
Friis S, Tadeo D, Le-Gall SM, Jürgensen HJ, Sales KU, Camerer E, Bugge TH. Matriptase zymogen supports epithelial development, homeostasis and regeneration. BMC Biol. 2017; 15(1): 46. doi: 10.1186/s12915-017-0384-4.

Friis S, Sales KU, Schafer JM, Vogel LK, Kataoka H, Bugge TH. The protease inhibitor HAI-2, but not HAI-1, regulates matriptase activation and shedding through prostasin. J Biol Chem.2014; 289: 22319-22332. doi:10.1074/jbc.M114.574400

Friis S, Uzzun Sales K, Godiksen S, Peters DE, Lin CY, Vogel LK, Bugge TH. A matriptase-prostasin reciprocal zymogen activation complex with unique features: prostasin as a non-enzymatic co-factor for matriptase activation. J Biol Chem. 2013; 288(26): 19028-39. doi: 10.1074/jbc.M113.469932.

Furuse M, Tsukita S. Claudins in occluding junctions of humans and flies. Trends Cell Biol. 2006; 16(4): 181-8. doi: 10.1016/j.tcb.2006.02.006

Gatza CE, Oh SY, Blobe GC. Roles for the type III TGF-beta receptor in human cancer. Cell Signal. 2010; 22(8): 1163-74. doi: 10.1016/j.cellsig.2010.01.016.

Genova JL, Fehon RG. Neuroglian, Gliotactin, and the Na+/K+ ATPase are essential for septate junction function in Drosophila. J Cell Biol. 2003; 161: 979-989. doi:10.1083/jcb.200212054

Ghabrial A, Luschnig S, Metzstein MM, Krasnow MA. Branching morphogenesis of the Drosophila tracheal system. Annu Rev Cell Dev Biol. 2003; 19: 623-47. doi: 10.1146/annurev.cellbio.19.031403.160043

Glasheen BM, Robbins RM, Piette C, Beitel GJ, Page-McCaw A. A matrix metalloproteinase mediates airway remodeling in Drosophila. Dev Biol. 2010; 344(2): 772-83. doi: 10.1016/j.ydbio.2010.05.504.

Gramates LS, Marygold SJ, Santos GD, Urbano JM, Antonazzo G, Matthews BB, Rey AJ, Tabone CJ, Crosby MA, Emmert DB, Falls K, Goodman JL, Hu Y, Ponting L, Schroeder AJ et al. the FlyBase Consortium. FlyBase at 25: looking to the future. Nucleic Acids Res. 2017; 45(D1): D663-D671. doi: 10.1093/nar/gkw1016.

Gratz SJ, Ukken FP, Rubinstein CD, Thiede G, Donohue LK, Cummings AM, O'Connor-Giles KM. Highly specific and efficient CRISPR/Cas9-catalyzed homology-directed repair in Drosophila. Genetics. 2014; 196(4): 961-71. doi: 10.1534/genetics.113.160713.

Gupta SK. Role of zona pellucida glycoproteins during fertilization in humans. J Reprod Immunol. 2015; 108: 90-7. doi: 10.1016/j.jri.2014.08.006. 
Hannezo E, Dong B, Recho P, Joanny JF, Hayashi S. Cortical instability drives periodic supracellular actin pattern formation in epithelial tubes. Proc Natl Acad Sci U S A. 2015; 112(28): 8620-5. doi: 10.1073/pnas.1504762112.

Hayashi S, Kondo T. Development and Function of the Drosophila Tracheal System. Genetics. 2018; 209(2): 367-380. doi: 10.1534/genetics.117.300167.

Hijazi A, Masson W, Augé B, Waltzer L, Haenlin M, Roch F. boudin is required for septate junction organisation in Drosophila and codes for a diffusible protein of the Ly6 superfamily. Development. 2009; 136(13): 2199-209. doi: 10.1242/dev.033845.

Hildebrandt A, Pflanz R, Behr M, Tarp T, Riedel D, Schuh R. Bark beetle controls epithelial morphogenesis by septate junction maturation in Drosophila. Dev Biol. 2015; 400(2): 237-47. doi: 10.1016/j.ydbio.2015.02.008

Hooper JD, Clements JA, Quigley JP, Antalis TM. Type II transmembrane serine proteases. Insights into an emerging class of cell surface proteolytic enzymes. J Biol Chem. 2001; 276: 857-860. doi:10.1074/jbc.R000020200

Hooper SB, Kitchen MJ, Siew ML, Lewis RA, Fouras A, te Pas AB, Siu KK, Yagi N, Uesugi K, Wallace MJ. Imaging lung aeration and lung liquid clearance at birth using phase contrast X-ray imaging. Clin Exp Pharmacol Physiol. 2009 ; 36(1): 117-25.

Huang J, Zhou W, Dong W, Watson AM, Hong Y. From the Cover: Directed, efficient, and versatile modifications of the Drosophila genome by genomic engineering. Proc Natl Acad Sci U S A. 2009; 106(20): 8284-9. doi: 10.1073/pnas.0900641106.

Hummler E, Planès C.Importance of ENaC-mediated sodium transport in alveolar fluid clearance using genetically-engineered mice. Cell Physiol Biochem. 2010; 25(1): 63-70. doi: 10.1159/000272051.

Inouye K, Tsuzuki S, Yasumoto M, Kojima K, Mochida S, Fushiki T. Identification of the matriptase second CUB domain as the secondary site for interaction with hepatocyte growth factor activator inhibitor type-1. J Biol Chem. 2010; 285(43): 33394-403. doi: 10.1074/jbc.M110.115816.

Isaac DD, Andrew DJ. Tubulogenesis in Drosophila: a requirement for the trachealess gene product. Genes Dev. 1996; 10(1): 103-17.

Itakura Y, Inagaki S, Wada H, Hayashi S. Trynity controls epidermal barrier function and respiratory tube maturation in Drosophila by modulating apical extracellular matrix nano-patterning. PLoS One. 2018; 13(12): e0209058. doi: 10.1371/journal.pone.0209058. 
Izumi Y, Furuse M. Molecular organization and function of invertebrate occluding junctions. Semin Cell Dev Biol.2014; 36: 186-93. doi: 10.1016/j.semcdb.2014.09.009.

Jarecki J, Johnson E, Krasnow MA. Oxygen regulation of airway branching in Drosophila is mediated by branchless FGF. Cell. 1999; 99(2): 211-20.

Jaspers MHJ, Pflanz R, Riedel D, Kawelke S, Feussner I, Schuh R. The fatty acylCoA reductase Waterproof mediates airway clearance in Drosophila. Dev Biol. 2014; 385: 23-31. doi:10.1016/j.ydbio.2013.10.022

Jaspers, MHJ. Identification and characterization of genes involved in tracheal liquid clearance of Drosophila melanogaster. 2011; PhD Thesis, Universität Osnabrück, Osnabrück.

Jaźwińska A, Ribeiro C, Affolter M. Epithelial tube morphogenesis during Drosophila tracheal development requires Piopio, a luminal ZP protein. Nat Cell Biol. 2003; 5: 895-901. doi:10.1038/ncb1049

Jin X, Yagi M, Akiyama N, Hirosaki T, Higashi S, Lin CY, Dickson RB, Kitamura H, Miyazaki K. Matriptase activates stromelysin (MMP-3) and promotes tumor growth and angiogenesis. Cancer Sci. 2006; 97(12): 1327-34. doi: 10.1111/j.13497006.2006.00328.x

Jovine L, Darie CC, Litscher ES, Wassarman PM. Zona pellucida domain proteins. Annu Rev Biochem. 2005; 74: 83-114.

Jovine L, Qi H, Williams Z, Litscher E, Wassarman PM. The ZP domain is a conserved module for polymerization of extracellular proteins. Nat Cell Biol. 2002; 4: 457-461. doi:10.1038/ncb802

Kato K, Chihara T, Hayashi S. Hedgehog and Decapentaplegic instruct polarized growth of cell extensions in the Drosophila trachea. Development. 2004; 131(21): 5253-61. doi: 10.1242/dev.01404

Kerman BE, Cheshire AM, Andrew DJ. From fate to function: the Drosophila trachea and salivary gland as models for tubulogenesis. Differentiation. 2006; 74(7): 326-48.

Ko CJ, Huang CC, Lin HY, Juan CP, Lan SW, Shyu HY, Wu SR, Hsiao PW, Huang HP, Shun CT, Lee MS. Androgen-Induced TMPRSS2 Activates Matriptase and Promotes Extracellular Matrix Degradation, Prostate Cancer Cell Invasion, Tumor Growth, and Metastasis. Cancer Res. 2015; 75(14): 2949-60. doi: 10.1158/0008-5472.CAN-14-3297.

Kondo S, Ueda R. Highly improved gene targeting by germline-specific cas9 expression in Drosophila. Genetics.2013; 195:715-721. doi:10.1534/genetics.113.156737 
Kondo T, Hayashi S. Mitotic cell rounding accelerates epithelial invagination. Nature. 2013; 494(7435): 125-9. doi: 10.1038/nature11792.

Krause C, Wolf C, Hemphälä J, Samakovlis C, Schuh R. Distinct functions of the leucine-rich repeat transmembrane proteins capricious and tartan in the Drosophila tracheal morphogenesis. Dev Biol. 2006; 296(1): 253-64. doi: 10.1016/j.ydbio.2006.04.462

Krogh A, Larsson B, von Heijne G, Sonnhammer EL. Predicting transmembrane protein topology with a hidden Markov model: application to complete genomes. Mol Biol. 2001; 305(3): 567-80. doi: 10.1006/jmbi.2000.4315

Lamb RS, Ward RE, Schweizer L, Fehon RG. Drosophila coracle, a member of the protein 4.1 superfamily, has essential structural functions in the septate junctions and developmental functions in embryonic and adult epithelial cells. Mol Biol Cell. 1998; 9: 3505-3519.

Lee M-S, Tseng I-C, Wang Y, Kiyomiya K-I, Johnson MD, Dickson RB, Lin CY. Autoactivation of matriptase in vitro: requirement for biomembrane and LDL receptor domain. Am J Physiol, Cell Physiol. 2007; 293: C95-105. doi:10.1152/ajpcell.00611.2006

Lee SP, Kao CY, Chang SC, Chiu YL, Chen YJ, Chen MG, Chang CC, Lin YW, Chiang CP, Wang JK, Lin CY, Johnson MD. Tissue distribution and subcellular localizations determine in vivo functional relationship among prostasin, matriptase, HAI-1, and HAI-2 in human skin. PLoS One. 2018; 13(2): e0192632. doi: 10.1371/journal.pone.0192632.

Leyvraz C, Charles RP, Rubera I, Guitard M, Rotman S, Breiden B, Sandhoff K, Hummler E. The epidermal barrier function is dependent on the serine protease CAP1/Prss8. J Cell Biol. 2005; 170(3): 487-96.

Li Q, Zhang Y, Pluchon P, Robens J, Herr K, Mercade M, Thiery JP, Yu H, Viasnoff V. Extracellular matrix scaffolding guides lumen elongation by inducing anisotropic intercellular mechanical tension. Nat Cell Biol. 2016; 18(3): 311-8. doi: $10.1038 /$ ncb3310.

Lin CY, Tseng IC, Chou FP, Su SF, Chen YW, Johnson MD, Dickson RB. Zymogen activation, inhibition, and ectodomain shedding of matriptase. Front Biosci. 2008; 13: 621-35.

Lin CY, Anders J, Johnson M, Sang QA, Dickson RB. Molecular cloning of cDNA for matriptase, a matrix-degrading serine protease with trypsin-like activity. $J$ Biol Chem. 1999; 274(26): 18231-6.

List K, Currie B, Scharschmidt TC, Szabo R, Shireman J, Molinolo A, Cravatt BF, Segre J, Bugge TH. Autosomal ichthyosis with hypotrichosis syndrome displays low matriptase proteolytic activity and is phenocopied in ST14 hypomorphic mice. J Biol Chem. 2007; 282(50): 36714-23. 
List K, Bugge TH, Szabo R. Matriptase: potent proteolysis on the cell surface. Mol Med. 2006; 12(1-3): 1-7. doi: 10.2119/2006-00022.List

List K, Szabo R, Wertz PW, Segre J, Haudenschild CC, Kim S-Y, et al. Loss of proteolytically processed filaggrin caused by epidermal deletion of Matriptase/MT-SP1. J Cell Biol. 2003; 163: 901-910. doi:10.1083/jcb.200304161

List K, Haudenschild CC, Szabo R, Chen W, Wahl SM, Swaim W, et al. Matriptase/MT-SP1 is required for postnatal survival, epidermal barrier function, hair follicle development, and thymic homeostasis. Oncogene. 2002; 21: 3765-3779. doi:10.1038/sj.onc.1205502

Liu L, Johnson WA, Welsh MJ. Drosophila DEG/ENaC pickpocket genes are expressed in the tracheal system, where they may be involved in liquid clearance. Proc Natl Acad Sci U S A. 2003; 100(4): 2128-33. doi: 10.1073/pnas.252785099

Llimargas M, Strigini M, Katidou M, Karagogeos D, Casanova J. Lachesin is a component of a septate junction-based mechanism that controls tube size and epithelial integrity in the Drosophila tracheal system. Development. 2004; 131(1): 181-90. doi: 10.1242/dev.00917

Luschnig S1, Bätz T, Armbruster K, Krasnow MA. serpentine and vermiform encode matrix proteins with chitin binding and deacetylation domains that limit tracheal tube length in Drosophila. Curr Biol. 2006; 16(2): 186-94. doi: 10.1016/j.cub.2005.11.072

Lye CM, Naylor HW, Sanson B. Subcellular localisations of the CPTI collection of YFP-tagged proteins in Drosophila embryos. Development. 2014; 141: 40064017. doi:10.1242/dev.111310

Manning G, Krasnow MA. Development of the Drosophila tracheal system. In: Bate M, Martínez- Arias A, editors. The development of Drosophila melanogaster. Cold Spring Harbor, NY: Cold Spring Harbor Laboratory Press. 1993; p 609685.

Maruyama R, Andrew DJ. Drosophila as a model for epithelial tube formation. Dev Dyn. 2012; 241(1): 119-35. doi: 10.1002/dvdy.22775.

Matalon S, Bartoszewski R, Collawn JF. Role of epithelial sodium channels in the regulation of lung fluid homeostasis. Am J Physiol Lung Cell Mol Physiol. 2015; 309(11): L1229-38. doi: 10.1152/ajplung.00319.2015.

Matusek T, Djiane A, Jankovics F, Brunner D, Mlodzik M, Mihály J. The Drosophila formin DAAM regulates the tracheal cuticle pattern through organizing the actin cytoskeleton. Development. 2006; 133(5): 957-66. 
Miao G, Hayashi S. Escargot controls the sequential specification of two tracheal tip cell types by suppressing FGF signaling in Drosophila. Development. 2016; 143(22): 4261-4271. doi: 10.1242/dev.133322

Miller GS, List K. The matriptase-prostasin proteolytic cascade in epithelial development and pathology. Cell Tissue Res. 2013; 351: 245-253. doi:10.1007/s00441-012-1348-1

Milner JM, Patel A, Davidson RK, Swingler TE, Desilets A, Young DA, et al. Matriptase is a novel initiator of cartilage matrix degradation in osteoarthritis. Arthritis Rheum. 2010; 62: 1955-1966. doi:10.1002/art.27476

Mitchell AC, Kannan D, Hunter SA, Parra Sperberg RA, Chang CH, Cochran JR. Engineering a potent inhibitor of matriptase from the natural hepatocyte growth factor activator inhibitor type-1 (HAI-1) protein. J Biol Chem. 2018; 293(14): 4969-4980. doi: 10.1074/jbc.M117.815142.

Moussian B, Letizia A, Martínez-Corrales G, Rotstein B, Casali A, Llimargas M. Deciphering the genetic programme triggering timely and spatially-regulated chitin deposition. PLoS Genet.2015; 11(1): e1004939. doi: 10.1371/journal.pgen.1004939.

Moussian B. The apical plasma membrane of chitin-synthesizing epithelia. Insect Sci. 2013; 20(2): 139-46. doi: 10.1111/j.1744-7917.2012.01549.x.

Moussian B. Recent advances in understanding mechanisms of insect cuticle differentiation. Insect Biochem Mol Biol. 2010; 40(5): 363-75. doi: 10.1016/j.ibmb.2010.03.003.

Moussian B, Seifarth C, Müller U, Berger J, Schwarz H. Cuticle differentiation during Drosophila embryogenesis. Arthr Struc \& Dev. 2006a; 35(3): 137-52

Moussian B, Tång E, Tonning A, Helms S, Schwarz H, Nüsslein-Volhard C, Uv AE. Drosophila Knickkopf and Retroactive are needed for epithelial tube growth and cuticle differentiation through their specific requirement for chitin filament organization. Development. 2006b; 133(1): 163-71. doi: 10.1242/dev.02177

Moussian B, Schwarz H, Bartoszewski S, Nüsslein-Volhard C. Involvement of chitin in exoskeleton morphogenesis in Drosophila melanogaster. J Morphol. 2005; 264(1): 117-30. doi: 10.1002/jmor.10324

Muñoz-Chápuli R. Evolution of angiogenesis. Int J Dev Biol. 2011; 55(4-5): 345-51. doi: 10.1387/ijdb.103212rm.

Myerburg MM, McKenna EE, Luke CJ, Frizzell RA, Kleyman TR, Pilewski JM. Prostasin expression is regulated by airway surface liquid volume and is increased in cystic fibrosis. Am J Physiol Lung Cell Mol Physiol. 2008; 294(5): L932-41. doi: 10.1152/ajplung.00437.2007. 
Nelson KS, Furuse M, Beitel GJ. The Drosophila Claudin Kune-kune Is Required for Septate Junction Organization and Tracheal Tube Size Control. Genetics. 2010; 185(3): 831-839. doi: [10.1534/genetics.110.114959]

Netzel-Arnett S, Currie BM, Szabo R, Lin CY, Chen LM, Chai KX, Antalis TM, Bugge TH, List K. Evidence for a matriptase-prostasin proteolytic cascade regulating terminal epidermal differentiation. J Biol Chem. 2006; 281(44): 32941-5.

Nielsen H. Predicting Secretory Proteins with SignalP. Methods Mol Biol. 2017; 1611: 59-73. doi: 10.1007/978-1-4939-7015-5_6.

Nonboe AW, Krigslund O, Soendergaard C, Skovbjerg S, Friis S, Andersen MN, Ellis V, Kawaguchi M, Kataoka H, Bugge TH, Vogel LK. HAI-2 stabilizes, inhibits and regulates SEA-cleavage-dependent secretory transport of matriptase. Traffic. 2017; 18: 378-391. doi:10.1111/tra.12482

Nüsslein-Volhard C, Wieschaus E, Kluding H. Mutations affecting the pattern of the larval cuticle in Drosophila melanogaster: I. Zygotic loci on the second chromosome. Wilehm Roux Arch Dev Biol. 1984; 193: 267-282. doi:10.1007/BF00848156

Oberst MD, Chen LY, Kiyomiya K, Williams CA, Lee MS, Johnson MD, Dickson $\mathrm{RB}$, Lin CY. HAI-1 regulates activation and expression of matriptase, a membrane-bound serine protease. Am J Physiol Cell Physiol. 2005; 289(2): C462-70. doi: 10.1152/ajpcell.00076.2005

Ochoa-Espinosa A, Affolter M. Branching morphogenesis: from cells to organs and back. Cold Spring Harb Perspect Biol. 2012; 4(10). pii: a008243. doi: 10.1101/cshperspect.a008243.

Öztürk-Çolak A, Stephan-Otto Attolini C, Casanova J, Araújo SJ. Blimp-1 Mediates Tracheal Lumen Maturation in Drosophila melanogaster. Genetics. 2018; 210(2): 653-663. doi: 10.1534/genetics.118.301444.

Öztürk-Çolak A, Moussian B, Araújo SJ. Drosophila chitinous aECM and its cellular interactions during tracheal development. Dev Dyn. 2016a; 245(3): 25967. doi: 10.1002/dvdy.24356.

Öztürk-Çolak A, Moussian B, Araújo SJ, Casanova J. A feedback mechanism converts individual cell features into a supracellular ECM structure in Drosophila trachea. Elife. 2016b; 5: pii: e09373. doi: 10.7554/eLife.09373.

Oh SY1, Knelson EH, Blobe GC, Mythreye K. The type III TGFB receptor regulates filopodia formation via a Cdc42-mediated IRSp53-N-WASP interaction in epithelial cells. Biochem J. 2013; 454(1): 79-89. doi: 10.1042/BJ20121701. 
Ohshiro T, Saigo K. Transcriptional regulation of breathless FGF receptor gene by binding of TRACHEALESS/dARNT heterodimers to three central midline elements in Drosophila developing trachea. Development. 1997; 124(20): 397586.

Ovaere P, Lippens S, Vandenabeele P, Declercq W. The emerging roles of serine protease cascades in the epidermis. Trends Biochem Sci. 2009; 34(9): 453-63. doi: 10.1016/j.tibs.2009.08.001. Epub 2009 Aug 31.

Pawar NR, Buzza MS, Antalis TM. Membrane-Anchored Serine Proteases and Protease-Activated Receptor-2-Mediated Signaling: Co-Conspirators in Cancer Progression. Cancer Res. 2019. doi: 10.1158/0008-5472.CAN-18-1745.

Philip A, Hannah R, O'connor-McCourt M. Ectodomain cleavage and shedding of the type III transforming growth factor-beta receptor in lung membranes effect of temperature, ligand binding and membrane solubilization. Eur J Biochem. 1999; 261(3): 618-28.

Polgár L. The catalytic triad of serine peptidases. Cell Mol Life Sci. 2005; 62: 21612172. doi:10.1007/s00018-005-5160-x

Ponce-Castañeda MV, Esparza-López J, Vilchis-Landeros MM, Mendoza V, LópezCasillas F. Murine betaglycan primary structure, expression and glycosaminoglycan attachment sites. Biochim Biophys Acta. 1998; 1384(2): 18996.

Qiu D, Owen K, Gray K, Bass R, Ellis V. Roles and regulation of membraneassociated serine proteases. Biochem Soc Trans. 2007; 35( 3): 583-7.

Reed KE, Grakoui A, Rice CM. Hepatitis C virus-encoded NS2-3 protease: cleavage-site mutagenesis and requirements for bimolecular cleavage. J Virol. 1995; 69(7): 4127-36.

Ribeiro C, Neumann M, Affolter M. Genetic control of cell intercalation during tracheal morphogenesis in Drosophila. Curr Biol. 2004; 14(24): 2197-207.

Rosa JB, Metzstein MM, Ghabrial AS. An Ichor-dependent apical extracellular matrix regulates seamless tube shape and integrity. PLoS Genet. 2018; 14: e1007146. doi:10.1371/journal.pgen.1007146

Ross J, Jiang H, Kanost MR, Wang Y. Serine proteases and their homologs in the Drosophila melanogaster genome: an initial analysis of sequence conservation and phylogenetic relationships. Gene. 2003; 304: 117-31.

Rubin GM, Spradling AC. Genetic transformation of Drosophila with transposable element vectors. Science. 1982; 218(4570): 348-53. 
Sakaidani Y, Nomura T, Matsuura A, Ito M, Suzuki E, Murakami K, Nadano D, Matsuda T, Furukawa K, Okajima T. O-linked-N-acetylglucosamine on extracellular protein domains mediates epithelial cell-matrix interactions. Nat Commun. 2011; 2: 583. doi: 10.1038/ncomms1591

Sales KU, Masedunskas A, Bey AL, Rasmussen AL, Weigert R, List K, Szabo R, Overbeek PA, Bugge TH. Matriptase initiates activation of epidermal prokallikrein and disease onset in a mouse model of Netherton syndrome. Nat Genet. 2010; 42(8): 676-83. doi: 10.1038/ng.629.

Sarov M, Barz C, Jambor H, Hein MY, Schmied C, Suchold D, Stender B, Janosch S, K J VV, Krishnan RT, Krishnamoorthy A, Ferreira IR, Ejsmont RK, Finkl K, Hasse S, et al. A genome-wide resource for the analysis of protein localisation in Drosophila. Elife. 2016; 5: e12068. doi: 10.7554/eLife.12068.

Satomi S, Yamasaki Y, Tsuzuki S, Hitomi Y, Iwanaga T, Fushiki T. A role for membrane-type serine protease (MT-SP1) in intestinal epithelial turnover. Biochem Biophys Res Commun.2001; 287(4) :995-1002. doi: 10.1006/bbrc.2001.5686

Schepis A, Barker A, Srinivasan Y, Balouch E, Zheng Y, Lam I, Clay H, Hsiao CD, Coughlin SR. Protease signaling regulates apical cell extrusion, cell contacts, and proliferation in epithelia. J Cell Biol. 2018; 217(3): 1097-1112. doi: $10.1083 /$ jcb.201709118.

Schaeffer C1, Santambrogio S, Perucca S, Casari G, Rampoldi L. Analysis of uromodulin polymerization provides new insights into the mechanisms regulating ZP domain-mediated protein assembly. Mol Biol Cell. 2009; 20(2): 589-99. doi: 10.1091/mbc.E08-08-0876.

Schottenfeld J, Song Y, Ghabrial AS. Tube continued: morphogenesis of the Drosophila tracheal system. Curr Opin Cell Biol. 2010; 22(5): 633-9. doi: 10.1016/j.ceb.2010.07.016.

Sievers F, Wilm A, Dineen D, Gibson TJ, Karplus K, Li W, Lopez R, McWilliam H, Remmert M, Söding J, Thompson JD, Higgins DG. Fast, scalable generation of high-quality protein multiple sequence alignments using Clustal Omega. Mol Syst Biol. 2011; 7: 539. doi: 10.1038/msb.2011.75.

Schindelin J, Arganda-Carreras I, Frise E, Kaynig V, Longair M, Pietzsch T, et al. Fiji: an open-source platform for biological-image analysis. Nat Methods. 2012; 9: 676-682. doi:10.1038/NMETH.2019

Steentoft C, Vakhrushev SY, Joshi HJ, Kong Y, Vester-Christensen MB, Schjoldager KT, Lavrsen K, Dabelsteen S, Pedersen NB, Marcos-Silva L, Gupta R, Bennett EP, Mandel U, Brunak S, Wandall HH, Levery SB, Clausen H. Precision mapping of the human O-GalNAc glycoproteome through SimpleCell technology. EMBO J, 2013; 32(10): 1478-88. doi: 10.1038/emboj.2013.79. 
Straus MR, Whittaker GR. A peptide-based approach to evaluate the adaptability of influenza A virus to humans based on its hemagglutinin proteolytic cleavage site. PLoS One. 2017; 12(3): e0174827. doi: 10.1371/journal.pone.0174827.

Strausberg RL, Feingold EA, Grouse LH, Derge JG, Klausner RD, Collins FS, Wagner L, Shenmen CM, Schuler GD, Altschul SF, Zeeberg B, Buetow KH, Schaefer CF, Bhat NK, Hopkins RF et al. Generation and initial analysis of more than 15,000 full-length human and mouse cDNA sequences. Proc Natl Acad Sci U S A. 2002; 99(26): 16899-903. doi: 10.1073/pnas.242603899

Subramanian A, Prokop A, Yamamoto M, Sugimura K, Uemura T, Betschinger J, Knoblich JA, Volk T. Shortstop recruits EB1/APC1 and promotes microtubule assembly at the muscle-tendon junction. Curr Biol. 2003; 13(13): 1086-95.

Sutherland D, Samakovlis C, Krasnow MA. branchless encodes a Drosophila FGF homolog that controls tracheal cell migration and the pattern of branching. Cell. 1996; 87(6): 1091-101.

Szabo L, Morey R, Palpant NJ, Wang PL, Afari N, Jiang C, Parast MM, Murry CE, Laurent LC, Salzman J. Statistically based splicing detection reveals neural enrichment and tissue-specific induction of circular RNA during human fetal development. Genome Biol. 2015; 16: 126. doi: 10.1186/s13059-015-0690-5.

Szabo R, Peters DE, Kosa P, Camerer E, Bugge TH. Regulation of feto-maternal barrier by matriptase- and PAR-2-mediated signaling is required for placental morphogenesis and mouse embryonic survival. PLoS Genet. 2014; 10(7): e1004470. doi: 10.1371/journal.pgen.1004470.

Szabo R, Bugge TH. Membrane-anchored serine proteases in vertebrate cell and developmental biology. Annu Rev Cell Dev Biol. 2011; 27: 213-35. doi: 10.1146/annurev-cellbio-092910-154247.

Tanabe LM, List K. The role of type II transmembrane serine protease-mediated signaling in cancer. FEBS J. 2017; 284(10): 1421-1436. doi: 10.1111/febs.13971.

Tautz D, Pfeifle C. A non-radioactive in situ hybridization method for the localization of specific RNAs in Drosophila embryos reveals translational control of the segmentation gene hunchback. Chromosoma. 1989; 98(2): 81-5.

Tepass U, Tanentzapf G, Ward R, Fehon R. Epithelial cell polarity and cell junctions in Drosophila. Annu Rev Genet. 2001; 35: 747-84.

Tiklová K, Tsarouhas V, Samakovlis C. Control of airway tube diameter and integrity by secreted chitin-binding proteins in Drosophila. PLoS One. 2013; 8(6): e67415. doi: 10.1371/journal.pone.0067415. 
Todorović-Raković N, Milovanović J, Nikolić-Vukosavljević D. TGF-B and its coreceptors in cancerogenesis: an overview. Biomark Med. 2011; 5(6): 855-63. doi: $10.2217 / \mathrm{bmm} .11 .59$.

Tonning A, Hemphälä J, Tång E, Nannmark U, Samakovlis C, Uv A. A transient luminal chitinous matrix is required to model epithelial tube diameter in the Drosophila trachea. Dev Cell.2005; 9(3): 423-30. doi : 10.1016/j.devcel.2005.07.012

Tsarouhas V, Senti KA, Jayaram SA, Tiklová K, Hemphälä J, Adler J, Samakovlis C. Sequential pulses of apical epithelial secretion and endocytosis drive airway maturation in Drosophila. Dev Cell.2007; 13(2):214-25. doi: 10.1016/j.devcel.2007.06.008

Tseng CC, Jia B, Barndt R, Gu Y, Chen CY, Tseng IC, Su SF, Wang JK, Johnson $\mathrm{MD}$, Lin CY. Matriptase shedding is closely coupled with matriptase zymogen activation and requires de novo proteolytic cleavage likely involving its own activity. PLoS One. 2017; 12(8): e0183507. doi: 10.1371/journal.pone.0183507

Tsuzuki S, Murai N, Miyake Y, Inouye K, Fushiki T. The structural requirements of matriptase in its ectodomain release in polarized epithelial cells. Biosci Biotechnol Biochem. 2010; 74(6): 1295-7.

Usui T, Shima Y, Shimada Y, Hirano S, Burgess RW, Schwarz TL, Takeichi M, Uemura T. Flamingo, a seven-pass transmembrane cadherin, regulates planar cell polarity under the control of Frizzled. Cell. 1999; 98(5): 585-95.

Uv A, Moussian B. The apical plasma membrane of Drosophila embryonic epithelia. Eur J Cell Biol. 2010; 89(2-3): 208-11. doi: 10.1016/j.ejcb.2009.11.009.

Van den Steen PE, Opdenakker G, Wormald MR, Dwek RA, Rudd PM. Matrix remodelling enzymes, the protease cascade and glycosylation. Biochim Biophys Acta. 2001;1528(2-3): 61-73.

Vander Ark A, Cao J, Li X. TGF-B receptors: In and beyond TGF-B signaling. Cell Signal. 2018; 52: 112-120. doi: 10.1016/j.cellsig.2018.09.002.

Verhoeven K, Van Laer L, Kirschhofer K, Legan PK, Hughes DC, Schatteman I, Verstreken M, Van Hauwe P, Coucke P, Chen A, Smith RJ, Somers T, Offeciers FE, Van de Heyning P, Richardson GP et al. Mutations in the human alphatectorin gene cause autosomal dominant non-syndromic hearing impairment. Nat Genet. 1998; 19(1): 60-2. doi: 10.1038/ng0598-60

Wang XF, Lin HY, Ng-Eaton E, Downward J, Lodish HF, Weinberg RA. Expression cloning and characterization of the TGF-beta type III receptor. Cell. 1991; 67(4): 797-805. 
Wang Y, Berger J, Moussian B. Trynity models a tube valve in the Drosophila larval airway system. Dev Biol 2018; 437(2): 75-83. doi: 10.1016/j.ydbio.2018.02.019

Webb SL, Sanders AJ, Mason MD, Jiang WG. Type II transmembrane serine protease (TTSP) deregulation in cancer. Front Biosci. 2011; 16:539-52.

Wigglesworth VB. The properties of the lining membrane of the insect tracheal system. Tissue Cell. 1990; 22(2): 231-8.

Wilk R, Weizman I, Shilo BZ. trachealess encodes a bHLH-PAS protein that is an inducer of tracheal cell fates in Drosophila. Genes Dev. 1996; 10(1): 93-102.

Wilkin MB, Becker MN, Mulvey D, Phan I, Chao A, Cooper K, et al. Drosophila dumpy is a gigantic extracellular protein required to maintain tension at epidermal-cuticle attachment sites. Curr Biol. 2000; 10: 559-567.

White ES. Lung extracellular matrix and fibroblast function. Ann Am Thorac Soc. 2015; 12(1): S30-3. doi: 10.1513/AnnalsATS.201406-240MG.

Wolf C, Gerlach N, Schuh R. Drosophila tracheal system formation involves FGFdependent cell extensions contacting bridge-cells. EMBO Rep. 2002; 3(6): 563-8. doi: 10.1093/embo-reports/kvf115

Wu CJ, Feng X, Lu M, Morimura S, Udey MC. Matriptase-mediated cleavage of EpCAM destabilizes claudins and dysregulates intestinal epithelial homeostasis. J Clin Invest. 2017; 127(2): 623-634. doi: 10.1172/JCI88428.

Wu SR, Teng CH, Tu YT, Ko CJ, Cheng TS, Lan SW, Lin HY, Lin HH, Tu HF, Hsiao PW, Huang HP, Chen CH, Lee MS. The Kunitz Domain I of Hepatocyte Growth Factor Activator Inhibitor-2 Inhibits Matriptase Activity and Invasive Ability of Human Prostate Cancer Cells. Sci Rep. 2017; 7(1): 15101. doi: 10.1038/s41598-017-15415-4.

Wu VM, Schulte J, Hirschi A, Tepass U, Beitel GJ. Sinuous is a Drosophila claudin required for septate junction organization and epithelial tube size control. J Cell Biol. 2004; 164(2): 313-323. doi: [10.1083/jcb.200309134]

Yamasaki K, Mukai S, Nagai T, Nakahara K, Fujii M, Terada N, Ohno A, Sato Y, Toda Y, Kataoka H, Kamoto T. Matriptase-Induced Phosphorylation of MET is Significantly Associated with Poor Prognosis in Invasive Bladder Cancer; an Immunohistochemical Analysis. Int J Mol Sci. 2018; 19(12). pii: E3708. doi: 10.3390/ijms19123708.

Zelzer E, Shilo BZ. Cell fate choices in Drosophila tracheal morphogenesis. Bi o es s ays.2000; 22(3): 219-26. doi : $10.1002 /$ ( S I C I) 1521 1878(200003)22:3<219::AID-BIES3>3.0.CO;2-A 
Zhang S, Sun WY, Wu JJ, Gu YJ, Wei W. Decreased expression of the type III TGF-B receptor enhances metastasis and invasion in hepatocellullar carcinoma progression. Oncol Rep. 2016; 35(4): 2373-81. doi: 10.3892/or.2016.4615.

Zoratti GL, Tanabe LM, Varela FA, Murray AS, Bergum C, Colombo É, Lang JE, Molinolo AA, Leduc R, Marsault E, Boerner J, List K. Targeting matriptase in breast cancer abrogates tumour progression via impairment of stromalepithelial growth factor signalling. Nat Commun. 2015; 6: 6776. doi: 10.1038/ncomms7776.

Zuo L, Iordanou E, Chandran RR, Jiang L. Novel mechanisms of tube-size regulation revealed by the Drosophila trachea. Cell Tissue Res. 2013; 354(2): 343-54. doi: 10.1007/s00441-013-1673-z. 


\section{Appendix}

\subsection{Synthesized double stranded DNA fragments}

1. Fragment of the tpr genomic region with an in-frame insertion of a mCherry encoding sequence 3 ' of the tpr ORF:

CGCCGGCGTTGTCTCGTGGGGCGAGGGCTGTGCCAAGGCCGGTTATCCTGGCGTCTATGCCAGA GTGAATCGCTACGGAACCTGGATCAAGAACCTCACCAAACAGGCTTGCCTCTGCCAGCAGGAGA CCAAGAAGATCAAGGGCATGGTGAGCAAAGGAGAAGAAGATAACATGGCAATCATCAAAGAATT TATGCGTTTCAAAGTGCACATGGAAGGTTCTGTGAACGGACACGAATTTGAAATTGAAGGTGAAG GTGAAGGCCGTCCTTATGAAGGAACACAAACGGCAAAGCTGAAAGTAACAAAAGGCGGACCGCT TCCGTTTGCATGGGATATCCTTTCTCCGCAATTCATGTACGGTTCAAAAGCATACGTGAAGCATC CGGCTGATATTCCTGATTATTTGAAGCTGTCATTCCCTGAAGGCTTCAAATGGGAGCGTGTGATG AACTTTGAAGATGGCGGTGTTGTTACTGTTACTCAAGATTCAAGCCTTCAAGACGGTGAATTTATT TACAAAGTGAAGCTGCGCGGAACAAACTTCCCATCTGACGGACCTGTCATGCAAAAGAAAACAAT GGGCTGGGAAGCAAGCTCTGAACGCATGTATCCAGAGGACGGTGCTTTAAAAGGAGAAATCAAA CAGCGTTTGAAGCTGAAAGACGGCGGACACTATGACGCTGAAGTGAAAACAACTTACAAAGCGA AAAAGCCGGTTCAGCTTCCAGGTGCTTACAACGTAAACATCAAACTTGATATTACAAGCCACAAT GAAGATTATACGATTGTTGAACAATATGAACGCGCTGAAGGCCGTCATTCAACTGGCGGAATGGA TGAGCTTTACAAATAGCGAACATCTGGCTATTTCTGTATCTATTAATAGTTCAATACTCTTGCTTG GCTGGCAATGTTTAATATTATATCAGAATTTGTATCTTCCATCCAATGTGAAAGACAAGTTTTCAT TACAAAAAGCTTGTTATTTCCAAAATCACTAATCTCATATGCCAAGAAACGAACTATTTTCGCCGA AAATAACTATGCAATTTCTTGGGATATATGCTCCAAAACTTTTTGCCACCCTTGCAAGAGTAAGTC AACTTCGGCTTCGTCGAAGATCCCATTTTCAAGCTGTTTTATATTTATTGATTAAAAGAAAAGTTT CCATAAAGTGCGCTAACCTTTCTCGGGAGCGGTACC

This fragment was cloned into the $p G E$-attB-tpr-rescue vector via NaeI (red) and KpnI (blue) restriction sites, generating the $p G E$-attB-tpr::mCherry vector.

\section{TwinStrep-tag encoding sequence and fragment of the $N p$ 3' UTR:}

TTCGCTAGCGGCGGTAGTGCATGGAGTCATCCGCAGTTTGAAAAGGGTGGCGGATCAGGAGGTG GCAGCGGTGGAAGTGCATGGAGCCATCCCCAATTCGAGAAGTAGGTCCCGCCCCGGCCCGACTC TTAGTGAATGCCTAGCACGTCTTC

This fragment was cloned into the $p G E$-attB-Np::GFP vector. The GFP encoding sequence was replaced with this fragment via NheI (red) and BmgBI (blue) restriction sites, generating the $p G E$-attB-Np::Strep vector. 
3. Fragment of the pio genomic region with mCherry encoding sequence inserted in the fourth exon of pio:

ACTGGCGATGGATTAGGCCAGGGACTTAATTTCGGCTTGTCGTGATTTATGTCAACGCTTTGTGC TTGAAAAGGGGTCGGGCATCCATGGTCCATGGTCGGACCTGATTGGATCGGATAGAGTAGCATA TATACCAACTATGCTAATGAGTGACTTGTCTCCACTTTCTTTGCAGACAATCGCAGCTCTGACGAC GCACGCGGCGCAGATTGGCGGCATGGTGAGCAAAGGAGAAGAAGATAACATGGCAATCATCAAA GAATTTATGCGTTTCAAAGTGCACATGGAAGGTTCTGTGAACGGACACGAATTTGAAATTGAAGG TGAAGGTGAAGGCCGTCCTTATGAAGGAACACAAACGGCAAAGCTGAAAGTAACAAAAGGCGGA CCGCTTCCGTTTGCATGGGATATCCTTTCTCCGCAATTCATGTACGGTTCAAAAGCATACGTGAA GCATCCGGCTGATATTCCTGATTATTTGAAGCTGTCATTCCCTGAAGGCTTCAAATGGGAGCGTG TGATGAACTTTGAAGATGGCGGTGTTGTTACTGTTACTCAAGATTCAAGCCTTCAAGACGGTGAA TTTATTTACAAAGTGAAGCTGCGCGGAACAAACTTCCCATCTGACGGACCTGTCATGCAAAAGAA AACAATGGGCTGGGAAGCAAGCTCTGAACGCATGTATCCAGAGGACGGTGCTTTAAAAGGAGAA ATCAAACAGCGTTTGAAGCTGAAAGACGGCGGACACTATGACGCTGAAGTGAAAACAACTTACAA AGCGAAAAAGCCGGTTCAGCTTCCAGGTGCTTACAACGTAAACATCAAACTTGATATTACAAGCC ACAATGAAGATTATACGATTGTTGAACAATATGAACGCGCTGAAGGCCGTCATTCAACTGGCGGA ATGGATGAGCTTTACAAAGGCGGCCCAACGGCAATGAGTGAGTACATAAATCTGTTCATTAGGAA GCTAAACCGAATTCTATGGATTGGGACACCGAGCCACTGGGGGCCCAAAGAAAAATTGCCAGCC AATTGGTAACGCAGACAGACGCTGGCAAGATTAA

This fragment was used as a template for PCRs with UAS-mCherry::pio-for / UASmCherry::pio-rev and pio-5'-arm2-for / pio-5'-arm2-rev primer pairs for generation of $p U A S T$-mCherry::pio and pHD-ScarlessDsRed-mCherry::pio vectors.

4. Fragment of the $N p$ genomic region containing a substitution of the serine 990 codon with an alanine codon:

CACCATATGCGAGTCCATGTACCGATCCGCGGGCTACATCGAGCACATACCGCACATATTCATCT GCGCCGGCTGGAAGAAGGGCGGGTACGACTCCTGCGAAGGTGAGTAAAAGGGCCAGATGCTGTG GACACCTCGGTAAACCAATGCTGTTGTCTTCCCCAGGCGATGCCGGTGGCCCCATGGTGCTGCAG CGTGAGTCCGACAAGCGTTTCCACCTCGGCGGTGTCATATCGTGGGGCATTGGATGTGCGGAGG CCAACCAGCCTGGCGTTTATACCCGCATCTCGGAATTCCGGGACTGGATCAACCAGATCCTGCAG TTCGCTAGCGGC

This fragment was cloned into the $p G E$-attB-Np::Strep vector via NdeI (red) and NheI (blue) restriction sites, generating the $p G E$-att $B-N p^{S 990 A}::$ Strep vector. 
5. 3 ' region of the $N p$ ORF followed by a NheI restriction site containing linker and TwinStrep-tag encoding sequence:

GAATTCCGGGACTGGATCAACCAGATCCTGCAGTTCGCTAGCGGCGGTAGTGCATGGAGTCATC CGCAGTTTGAAAAGGGTGGCGGATCAGGAGGTGGCAGCGGTGGAAGTGCATGGAGCCATCCCCA ATTCGAGAAGTAGCTCGAG

This fragment was cloned into the $p U A S T-N p$ vector via EcoRI (red) and XhoI (blue) restriction sites, generating the $p U A S T-N p:: S t r e p$ vector.

6. 3 ' region of the $N p$ ORF containing a substitution of the serine 990 codon with an alanine codon:

CCGCGGGCTACATCGAGCACATACCGCACATATTCATCTGCGCCGGCTGGAAGAAGGGCGGGTA CGACTCCTGCGAAGGCGATGCCGGTGGCCCCATGGTGCTGCAGCGTGAGTCCGACAAGCGTTTC CACCTCGGCGGTGTCATATCGTGGGGCATTGGATGTGCGGAGGCCAACCAGCCTGGCGTTTATA CCCGCATCTCGGAATTCCGGGACTGGATCAACCAGATCCTGCAGTTCTAGCTCGAG

This fragment was cloned into the $p U A S T$-Np vector via SacII (red) and XhoI (blue) restriction sites, generating the $p U A S T-N p^{S 990 A}$ vector.

7. $3^{\prime}$ region of the matriptase ORF containing a substitution of the serine 805 codon with an alanine codon:

GCATGCTCTGGGCCAGGGCCACATCTGCGGTGCTTCCCTCATCTCTCCCAACTGGCTGGTCTCTG CCGCACACTGCTACATCGATGACAGAGGATTCAGGTACTCAGACCCCACGCAGTGGACGGCCTTC CTGGGCTTGCACGACCAGAGCCAGCGCAGCGCCCCTGGGGTGCAGGAGCGCAGGCTCAAGCGCA TCATCTCCCACCCCTTCTTCAATGACTTCACCTTCGACTATGACATCGCGCTGCTGGAGCTGGAG AAACCGGCAGAGTACAGCTCCATGGTGCGGCCCATCTGCCTGCCGGACGCCTCCCATGTCTTCCC TGCCGGCAAGGCCATCTGGGTCACGGGCTGGGGACACACCCAGTATGGAGGCACTGGCGCGCTG ATCCTGCAAAAGGGTGAGATCCGCGTCATCAACCAGACCACCTGCGAGAACCTCCTGCCGCAGC AGATCACGCCGCGCATGATGTGCGTGGGCTTCCTCAGCGGCGGCGTGGACTCCTGCCAGGGTGA TGCCGGGGGACCCCTGTCCAGCGTGGAGGCGGATGGGCGGATCTTCCAGGCCGGTGTGGTGAGC TGGGGAGACGGCTGCGCTCAGAGGAACAAGCCAGGCGTGTACACAAGGCTCCCTCTGTTTCGGG ACTGGATCAAAGAGAACACTGGGGTATAGTCTAGA

This fragment was cloned into the pUAST-matriptase vector via $\mathrm{SphI}$ (red) and $\mathrm{XbaI}$ (blue) restriction sites, generating the $p U A S T$-matriptase ${ }^{S 805 A}$ vector. 
8. $5^{\prime}$ region of the pio ORF with an in-frame insertion of a 3xFlag-tag encoding sequence:

GAATTCATGAAGACAGGCACTCGAATGGACGCTTTCCACACGGCGCTGCACTTAATCACAATCGC AGCTCTGACGACGCACGCGGCGCAGATTCCAACGGCAATGAAAGATGCCCAGAGCTCGCTGAGC GATGCGATTGCCGCGGCGGAGGCGGAGGTGGCCTCCACTAGTAAGGGCGACTACAAGGATCACG ACGGCGATTACAAAGACCACGATATCGACTACAAGGATGACGACGATAAGTCGCCGGC

This fragment was cloned into the $p U A S T$-mCherry::pio vector. mCherry encoding sequence was replaced with this fragment via EcoRI (red) and NaeI (blue) restriction sites, generating the $p U A S T$-Flag::pio vector.

\section{ORF of human HAI-1 that was codon optimized for $D$. melangaster:}

GAATTCATGGCACCGGCGAGGACAATGGCCCGTGCTCGACTGGCTCCTGCGGGAATACCAGCCGTGGC ACTGTGGTTGCTGTGCACCTTGGGCCTGCAGGGTACACAAGCCGGCCCTCCACCCGCTCCACCGGGTCT ACCAGCAGGAGCCGATTGCCTCAACTCGTTCACTGCTGGTGTTCCCGGATTTGTGCTGGACACTAATGC CTCCGTGTCTAATGGTGCCACGTTTCTGGAGAGTCCCACCGTTCGCAGGGGCTGGGACTGCGTTCGCGC TTGCTGTACGACGCAGAATTGCAATCTCGCGCTAGTGGAGCTGCAACCGGACAGAGGTGAAGATGCGA TCGCCGCCTGCTTCCTGATCAACTGCCTGTATGAGCAGAACTTCGTGTGCAAGTTTGCCCCAAGGGAGG GATTCATCAATTACCTTACCCGAGAAGTCTATCGGTCATACCGTCAGCTGAGGACCCAGGGTTTCGGAG GATCGGGCATTCCCAAGGCATGGGCCGGAATTGATCTCAAGGTGCAGCCCCAGGAGCCCCTTGTCCTTA AGGACGTAGAGAACACGGATTGGCGCTTGCTGCGAGGAGATACCGACGTCCGTGTCGAACGCAAAGAC CCGAATCAGGTTGAACTGTGGGGCCTGAAAGAGGGCACCTACTTGTTTCAGTTGACGGTGACTTCTTCG GACCATCCCGAGGATACGGCCAATGTTACCGTTACAGTCCTATCCACCAAACAGACAGAGGACTACTGT CTCGCCTCGAACAAGGTGGGCCGCTGTCGAGGCTCATTTCCGCGGTGGTACTACGATCCCACAGAGCAG ATTTGCAAGAGCTTTGTCTATGGCGGCTGTTTGGGCAACAAGAATAACTATCTCCGGGAGGAAGAGTGC ATCTTGGCCTGTCGTGGCGTACAAGGTGGTCCCCTGCGCGGAAGCAGTGGCGCTCAAGCCACCTTTCCA CAAGGCCCGAGCATGGAAAGACGCCATCCTGTTTGTAGCGGCACTTGCCAGCCAACTCAATTCCGCTGC TCTAATGGCTGCTGCATCGACAGCTTCCTGGAGTGCGACGATACCCCGAATTGCCCAGATGCCTCGGAT GAAGCGGCCTGTGAGAAATACACGAGTGGATTCGACGAGTTGCAGCGTATCCACTTTCCGTCCGACAA AGGTCACTGCGTGGATCTGCCCGATACGGGCCTTTGTAAGGAGTCCATACCCCGGTGGTACTACAACCC CTTCAGTGAACATTGCGCGCGCTTTACCTACGGAGGCTGCTATGGCAACAAGAACAACTTCGAGGAGG AACAGCAATGTCTCGAATCCTGCCGCGGCATTTCCAAGAAGGACGTGTTCGGCCTAAGACGGGAGATT CCGATACCTTCGACTGGATCAGTGGAGATGGCAGTGGCGGTGTTCCTGGTCATTTGCATCGTCGTAGTA GTGGCAATCCTGGGATATTGCTTCTTCAAGAACCAGCGCAAGGATTTCCATGGCCACCACCACCATCCA CCGCCGACACCCGCTAGCTCCACGGTGAGCACCACCGAGGATACCGAACACCTGGTGTACAACCACAC AACGCGACCTCTGTAAGGTACC

This fragment was cloned into the $p U A S T$ vector via EcoRI (red) and KpnI (blue) restriction sites, generating the $p U A S T-H A I-1$ vector. 
10. ORF of human HAI-2 that was codon optimized for D. melangaster:

GAATTCATGGCCCAACTGTGCGGTTTGCGTCGTAGCCGCGCTTTTCTCGCGTTGCTGGGCAGTCTGCTGC TTTCGGGAGTTCTAGCCGCAGATCGCGAACGCTCGATTCACGACTTCTGCCTGGTGAGCAAAGTCGTTG GACGCTGTAGAGCGTCCATGCCGAGATGGTGGTACAACGTGACCGATGGATCCTGCCAGCTGTTCGTGT ATGGTGGCTGCGATGGCAACAGTAATAACTACCTGACCAAAGAGGAGTGCCTCAAGAAGTGCGCCACT GTCACGGAGAATGCCACGGGTGACTTGGCCACTAGCCGGAATGCCGCCGATTCATCCGTGCCTTCAGCA CCAAGGCGACAGGACTCTGAGGACCATTCGTCCGATATGTTCAACTACGAGGAGTATTGCACCGCTAAT GCGGTTACAGGCCCATGTCGCGCTAGCTTTCCCCGATGGTACTTCGATGTAGAGCGTAATAGCTGCAAC AACTTCATCTATGGCGGCTGTAGGGGCAACAAGAACTCGTATCGCTCTGAAGAGGCCTGTATGCTCCGG TGCTTTCGCCAACAGGAAAATCCGCCCCTACCCCTGGGCAGCAAGGTCGTGGTTCTTGCAGGACTGTTT GTGATGGTGCTGATCCTGTTCCTCGGAGCCTCCATGGTGTACCTGATACGCGTAGCTCGTCGGAATCAG GAACGAGCGTTGAGGACAGTCTGGTCGAGTGGTGACGACAAGGAGCAGCTTGTGAAGAACACCTACGT CTTGTAAGGTACCGGTACC

This fragment was cloned into the $p U A S T$ vector via EcoRI (red) and KpnI (blue) restriction sites, generating the $p U A S T-H A I-2$ vector.

\subsection{Measurements of chitin strand diameters and frequencies}

E: embryo; D: diameter of chitin strands $(\mu \mathrm{m}) ; \mathrm{F}$ : chitin strands per $10 \mu \mathrm{m}$ DT length

\begin{tabular}{|c|c|c|c|c|c|c|c|c|c|c|c|c|c|c|c|c|c|c|c|}
\hline \multicolumn{20}{|c|}{ wild-type } \\
\hline \multicolumn{2}{|c|}{ E1 } & \multicolumn{2}{|c|}{ E2 } & \multicolumn{2}{|c|}{ E3 } & \multicolumn{2}{|c|}{ E4 } & \multicolumn{2}{|c|}{ E5 } & \multicolumn{2}{|c|}{ E6 } & \multicolumn{2}{|c|}{ E7 } & \multicolumn{2}{|c|}{ E8 } & \multicolumn{2}{|c|}{ E9 } & \multicolumn{2}{|c|}{ E10 } \\
\hline $\mathrm{D}$ & $\mathrm{F}$ & $\mathrm{D}$ & $\mathrm{F}$ & $\mathrm{D}$ & $\mathrm{F}$ & $\mathrm{D}$ & $\mathrm{F}$ & $\mathrm{D}$ & $\mathrm{F}$ & $\mathrm{D}$ & $\mathrm{F}$ & $\mathrm{D}$ & $\mathrm{F}$ & $\mathrm{D}$ & $\mathrm{F}$ & $\mathrm{D}$ & $\mathrm{F}$ & $\mathrm{D}$ & $\mathrm{F}$ \\
\hline 0,35 & \multirow{10}{*}{20} & 0,32 & \multirow{10}{*}{23} & 0,36 & \multirow{10}{*}{19} & 0,33 & \multirow{10}{*}{22} & 0,36 & \multirow{10}{*}{20} & 0,33 & \multirow{10}{*}{20} & 0,32 & \multirow{10}{*}{20} & 0,34 & \multirow{10}{*}{20} & 0,32 & \multirow{10}{*}{21} & 0,35 & \multirow{10}{*}{22} \\
\hline 0,32 & & 0,34 & & 0,35 & & 0,31 & & 0,35 & & 0,34 & & 0,30 & & 0,36 & & 0,34 & & 0,35 & \\
\hline 0,33 & & 0,32 & & 0,35 & & 0,32 & & 0,34 & & 0,33 & & 0,34 & & 0,31 & & 0,32 & & 0,31 & \\
\hline 0,30 & & 0,33 & & 0,29 & & 0,31 & & 0,33 & & 0,34 & & 0,32 & & 0,36 & & 0,34 & & 0,33 & \\
\hline 0,33 & & 0,30 & & 0,29 & & 0,28 & & 0,30 & & 0,29 & & 0,35 & & 0,31 & & 0,33 & & 0,37 & \\
\hline 0,35 & & 0,36 & & 0,35 & & 0,30 & & 0,31 & & 0,32 & & 0,36 & & 0,32 & & 0,32 & & 0,35 & \\
\hline 0,35 & & 0,34 & & 0,30 & & 0,30 & & 0,37 & & 0,33 & & 0,34 & & 0,32 & & 0,30 & & 0,36 & \\
\hline 0,33 & & 0,30 & & 0,37 & & 0,31 & & 0,28 & & 0,34 & & 0,36 & & 0,37 & & 0,35 & & 0,35 & \\
\hline 0,31 & & 0,34 & & 0,35 & & 0,35 & & 0,37 & & 0,37 & & 0,30 & & 0,31 & & 0,28 & & 0,37 & \\
\hline 0,34 & & 0,31 & & 0,30 & & 0,35 & & 0,32 & & 0,34 & & 0,34 & & 0,33 & & 0,31 & & 0,28 & \\
\hline 0,37 & & 0,30 & & 0,36 & & 0,29 & & 0,35 & & 0,37 & & 0,32 & & 0,35 & & 0,31 & & 0,35 & \\
\hline 0,33 & & 0,31 & & 0,33 & & 0,35 & & 0,31 & & 0,33 & & 0,30 & & 0,31 & & 0,32 & & 0,35 & \\
\hline 0,35 & & 0,36 & & 0,35 & & 0,33 & & 0,35 & & 0,35 & & 0,33 & & 0,33 & & 0,29 & & 0,29 & \\
\hline 0,32 & & 0,30 & & 0,34 & & 0,36 & & 0,36 & & 0,29 & & 0,33 & & 0,30 & & 0,28 & & 0,31 & \\
\hline 0,30 & 10 & 0,32 & 1 & 0,29 & סק & 0,29 & & 0,28 & 89 & 0,32 & & 0,30 & & 0,33 & & 0,36 & & 0,34 & \\
\hline 0,34 & 10 & 0,34 & 10 & 0,29 & 20 & 0,31 & 10 & 0,29 & 22 & 0,36 & 22 & 0,35 & 21 & 0,35 & 20 & 0,29 & 21 & 0,34 & 20 \\
\hline 0,36 & & 0,32 & & 0,29 & & 0,31 & & 0,30 & & 0,32 & & 0,34 & & 0,31 & & 0,37 & & 0,30 & \\
\hline 0,33 & & 0,31 & & 0,33 & & 0,35 & & 0,32 & & 0,34 & & 0,29 & & 0,28 & & 0,36 & & 0,30 & \\
\hline 0,31 & & 0,32 & & 0,29 & & 0,34 & & 0,32 & & 0,34 & & 0,33 & & 0,33 & & 0,36 & & 0,31 & \\
\hline 0,29 & & 0,37 & & 0,33 & & 0,31 & & 0,33 & & 0,31 & & 0,28 & & 0,36 & & 0,34 & & 0,30 & \\
\hline
\end{tabular}




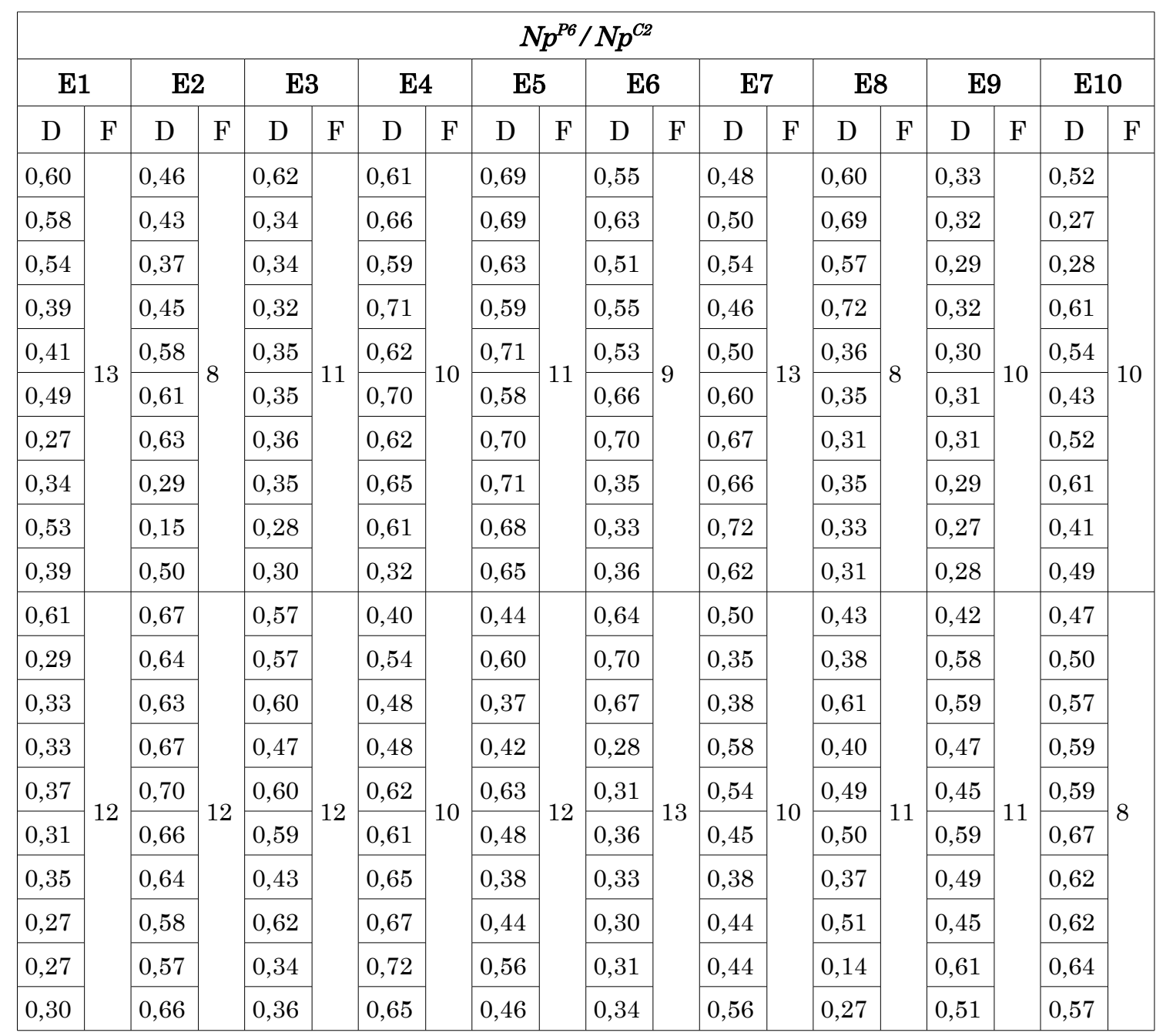

\begin{tabular}{|c|c|c|c|c|c|c|c|c|c|c|c|c|c|c|c|c|c|c|c|}
\hline \multicolumn{20}{|c|}{$N p^{P 6}, b t l-G a l 4 / N p^{C 2}, U A S-N p$} \\
\hline \multicolumn{2}{|c|}{ E1 } & \multicolumn{2}{|c|}{ E2 } & \multicolumn{2}{|c|}{ E3 } & \multicolumn{2}{|c|}{ E4 } & \multicolumn{2}{|c|}{ E5 } & \multicolumn{2}{|c|}{ E6 } & \multicolumn{2}{|c|}{ E7 } & \multicolumn{2}{|c|}{ E8 } & \multicolumn{2}{|c|}{ E9 } & \multicolumn{2}{|c|}{ E10 } \\
\hline $\mathrm{D}$ & $\mathrm{F}$ & $\mathrm{D}$ & $\mathrm{F}$ & $\mathrm{D}$ & $\mathrm{F}$ & $\mathrm{D}$ & $\mathrm{F}$ & $\mathrm{D}$ & $\mathrm{F}$ & $\mathrm{D}$ & $\mathrm{F}$ & $\mathrm{D}$ & $\mathrm{F}$ & $\mathrm{D}$ & $\mathrm{F}$ & $\mathrm{D}$ & $\mathrm{F}$ & $\mathrm{D}$ & $\mathrm{F}$ \\
\hline 0,34 & \multirow{10}{*}{20} & 0,34 & \multirow{10}{*}{22} & 0,30 & \multirow{10}{*}{20} & 0,32 & \multirow{10}{*}{22} & 0,32 & \multirow{10}{*}{21} & 0,33 & \multirow{10}{*}{20} & 0,31 & \multirow{10}{*}{23} & 0,29 & \multirow{10}{*}{22} & 0,28 & \multirow{10}{*}{20} & 0,30 & \multirow{10}{*}{21} \\
\hline 0,32 & & 0,27 & & 0,28 & & 0,28 & & 0,35 & & 0,34 & & 0,33 & & 0,32 & & 0,30 & & 0,31 & \\
\hline 0,29 & & 0,30 & & 0,30 & & 0,33 & & 0,35 & & 0,29 & & 0,30 & & 0,27 & & 0,30 & & 0,30 & \\
\hline 0,33 & & 0,31 & & 0,29 & & 0,34 & & 0,27 & & 0,30 & & 0,28 & & 0,27 & & 0,28 & & 0,28 & \\
\hline 0,35 & & 0,28 & & 0,27 & & 0,31 & & 0,27 & & 0,27 & & 0,30 & & 0,29 & & 0,29 & & 0,32 & \\
\hline 0,32 & & 0,28 & & 0,32 & & 0,30 & & 0,29 & & 0,33 & & 0,32 & & 0,30 & & 0,33 & & 0,28 & \\
\hline 0,28 & & 0,30 & & 0,31 & & 0,33 & & 0,35 & & 0,30 & & 0,32 & & 0,29 & & 0,29 & & 0,34 & \\
\hline 0,30 & & 0,32 & & 0,34 & & 0,35 & & 0,33 & & 0,30 & & 0,27 & & 0,28 & & 0,35 & & 0,29 & \\
\hline 0,34 & & 0,32 & & 0,33 & & 0,32 & & 0,31 & & 0,31 & & 0,29 & & 0,29 & & 0,31 & & 0,35 & \\
\hline 0,34 & & 0,28 & & 0,28 & & 0,27 & & 0,31 & & 0,32 & & 0,32 & & 0,31 & & 0,31 & & 0,30 & \\
\hline
\end{tabular}




\begin{tabular}{|l|l|l|l|l|l|l|l|l|l|l|l|l|l|l|l|}
\hline 0,32 \\
\hline 0,34
\end{tabular}

\begin{tabular}{|c|c|c|c|c|c|c|c|c|c|c|c|c|c|c|c|c|c|c|c|}
\hline \multicolumn{20}{|c|}{$N p^{P 6}, b t l-G a l 4 / N p^{C 2}, U A S-N p^{S 990 A}$} \\
\hline \multicolumn{2}{|c|}{ E1 } & \multicolumn{2}{|c|}{ E2 } & \multicolumn{2}{|c|}{ E3 } & \multicolumn{2}{|c|}{ E4 } & \multicolumn{2}{|c|}{ E5 } & \multicolumn{2}{|c|}{ E6 } & \multicolumn{2}{|c|}{ E7 } & \multicolumn{2}{|c|}{ E8 } & \multicolumn{2}{|c|}{ E9 } & \multicolumn{2}{|c|}{ E10 } \\
\hline $\mathrm{D}$ & $\mathrm{F}$ & $\mathrm{D}$ & $\mathrm{F}$ & $\mathrm{D}$ & $\mathrm{F}$ & $\mathrm{D}$ & $\mathrm{F}$ & $\mathrm{D}$ & $\mathrm{F}$ & $\mathrm{D}$ & $\mathrm{F}$ & $\mathrm{D}$ & $\mathrm{F}$ & $\mathrm{D}$ & $\mathrm{F}$ & $\mathrm{D}$ & $\mathrm{F}$ & $\mathrm{D}$ & $\mathrm{F}$ \\
\hline 0,33 & \multirow{10}{*}{10} & 0,54 & \multirow{10}{*}{11} & 0,35 & \multirow{10}{*}{11} & 0,63 & \multirow{10}{*}{13} & 0,54 & \multirow{10}{*}{9} & 0,49 & \multirow{10}{*}{13} & 0,48 & \multirow{10}{*}{10} & 0,37 & \multirow{10}{*}{12} & 0,38 & \multirow{10}{*}{13} & 0,58 & \multirow{10}{*}{11} \\
\hline 0,34 & & 0,53 & & 0,28 & & 0,36 & & 0,40 & & 0,42 & & 0,27 & & 0,36 & & 0,55 & & 0,28 & \\
\hline 0,63 & & 0,58 & & 0,58 & & 0,48 & & 0,36 & & 0,58 & & 0,46 & & 0,38 & & 0,28 & & 0,37 & \\
\hline 0,28 & & 0,39 & & 0,51 & & 0,36 & & 0,47 & & 0,68 & & 0,61 & & 0,39 & & 0,79 & & 0,48 & \\
\hline 0,30 & & 0,49 & & 0,60 & & 0,32 & & 0,40 & & 0,64 & & 0,30 & & 0,59 & & 0,49 & & 0,77 & \\
\hline 0,45 & & 0,32 & & 0,62 & & 0,54 & & 0,40 & & 0,69 & & 0,54 & & 0,57 & & 0,52 & & 0,55 & \\
\hline 0,32 & & 0,30 & & 0,46 & & 0,38 & & 0,51 & & 0,69 & & 0,47 & & 0,52 & & 0,51 & & 0,44 & \\
\hline 0,79 & & 0,56 & & 0,58 & & 0,34 & & 0,35 & & 0,79 & & 0,59 & & 0,39 & & 0,27 & & 0,59 & \\
\hline 0,34 & & 0,57 & & 0,43 & & 0,42 & & 0,77 & & 0,75 & & 0,74 & & 0,54 & & 0,28 & & 0,45 & \\
\hline 0,60 & & 0,47 & & 0,37 & & 0,33 & & 0,79 & & 0,63 & & 0,67 & & 0,29 & & 0,30 & & 0,54 & \\
\hline 0,59 & \multirow{10}{*}{12} & 0,33 & \multirow{10}{*}{9} & 0,66 & \multirow{10}{*}{11} & 0,39 & \multirow{10}{*}{11} & 0,54 & \multirow{10}{*}{8} & 0,72 & \multirow{10}{*}{10} & 0,63 & & 0,37 & & 0,37 & & 0,48 & \\
\hline 0,55 & & 0,60 & & 0,78 & & 0,57 & & 0,44 & & 0,70 & & 0,31 & & 0,42 & & 0,34 & & 0,59 & \\
\hline 0,41 & & 0,54 & & 0,76 & & 0,57 & & 0,56 & & 0,67 & & 0,61 & & 0,43 & & 0,61 & & 0,28 & \\
\hline 0,29 & & 0,32 & & 0,69 & & 0,51 & & 0,35 & & 0,68 & & 0,74 & & 0,51 & & 0,36 & & 0,57 & \\
\hline 0,46 & & 0,31 & & 0,59 & & 0,37 & & 0,38 & & 0,63 & & 0,68 & 11 & 0,41 & 11 & 0,49 & 10 & 0,32 & 10 \\
\hline 0,65 & & 0,28 & & 0,71 & & 0,34 & & 0,54 & & 0,61 & & 0,69 & 11 & 0,62 & 11 & 0,33 & 10 & 0,41 & 10 \\
\hline 0,61 & & 0,61 & & 0,59 & & 0,46 & & 0,59 & & 0,62 & & 0,75 & & 0,56 & & 0,28 & & 0,46 & \\
\hline 0,71 & & 0,60 & & 0,58 & & 0,31 & & 0,46 & & 0,77 & & 0,68 & & 0,57 & & 0,46 & & 0,48 & \\
\hline 0,73 & & 0,65 & & 0,69 & & 0,38 & & 0,77 & & 0,70 & & 0,59 & & 0,56 & & 0,50 & & 0,29 & \\
\hline 0,59 & & 0,72 & & 0,75 & & 0,36 & & 0,70 & & 0,59 & & 0,67 & & 0,59 & & 0,62 & & 0,34 & \\
\hline
\end{tabular}




\begin{tabular}{|c|c|c|c|c|c|c|c|c|c|c|c|c|c|c|c|c|c|c|c|}
\hline \multicolumn{20}{|c|}{$N p^{P 6}$, btl-Gal4 / Np $p^{C 2}, U A S$-matriptase } \\
\hline \multicolumn{2}{|c|}{ E1 } & \multicolumn{2}{|c|}{ E2 } & \multicolumn{2}{|c|}{ E3 } & \multicolumn{2}{|c|}{ E4 } & \multicolumn{2}{|c|}{ E5 } & \multicolumn{2}{|c|}{ E6 } & \multicolumn{2}{|c|}{ E7 } & \multicolumn{2}{|c|}{ E8 } & \multicolumn{2}{|c|}{ E9 } & \multicolumn{2}{|c|}{ E10 } \\
\hline $\mathrm{D}$ & $\mathrm{F}$ & $\mathrm{D}$ & $\mathrm{F}$ & $\mathrm{D}$ & $\mathrm{F}$ & $\mathrm{D}$ & $\mathrm{F}$ & $\mathrm{D}$ & $\mathrm{F}$ & $\mathrm{D}$ & $\mathrm{F}$ & $\mathrm{D}$ & $\mathrm{F}$ & $\mathrm{D}$ & $\mathrm{F}$ & $\mathrm{D}$ & $\mathrm{F}$ & $\mathrm{D}$ & $\mathrm{F}$ \\
\hline 0,34 & \multirow{10}{*}{18} & 0,34 & \multirow{10}{*}{17} & 0,34 & \multirow{10}{*}{21} & 0,35 & \multirow{10}{*}{21} & 0,29 & \multirow{10}{*}{20} & 0,32 & \multirow{10}{*}{16} & 0,28 & \multirow{10}{*}{20} & 0,30 & \multirow{10}{*}{21} & 0,29 & \multirow{10}{*}{20} & 0,33 & \multirow{10}{*}{17} \\
\hline 0,33 & & 0,31 & & 0,31 & & 0,30 & & 0,33 & & 0,31 & & 0,29 & & 0,34 & & 0,29 & & 0,29 & \\
\hline 0,30 & & 0,34 & & 0,32 & & 0,32 & & 0,28 & & 0,31 & & 0,30 & & 0,30 & & 0,30 & & 0,34 & \\
\hline 0,35 & & 0,32 & & 0,31 & & 0,30 & & 0,27 & & 0,35 & & 0,29 & & 0,30 & & 0,33 & & 0,31 & \\
\hline 0,34 & & 0,32 & & 0,33 & & 0,34 & & 0,30 & & 0,32 & & 0,34 & & 0,33 & & 0,30 & & 0,34 & \\
\hline 0,32 & & 0,31 & & 0,31 & & 0,30 & & 0,29 & & 0,34 & & 0,34 & & 0,35 & & 0,31 & & 0,30 & \\
\hline 0,31 & & 0,33 & & 0,32 & & 0,29 & & 0,30 & & 0,29 & & 0,32 & & 0,34 & & 0,28 & & 0,27 & \\
\hline 0,32 & & 0,30 & & 0,28 & & 0,29 & & 0,29 & & 0,33 & & 0,28 & & 0,31 & & 0,32 & & 0,29 & \\
\hline 0,34 & & 0,30 & & 0,33 & & 0,28 & & 0,30 & & 0,31 & & 0,34 & & 0,28 & & 0,29 & & 0,26 & \\
\hline 0,32 & & 0,34 & & 0,29 & & 0,29 & & 0,27 & & 0,27 & & 0,29 & & 0,32 & & 0,33 & & 0,26 & \\
\hline 0,29 & \multirow{10}{*}{18} & 0,33 & & 0,31 & & 0,34 & & 0,31 & & 0,32 & & 0,32 & & 0,28 & & 0,33 & & 0,31 & \\
\hline 0,32 & & 0,33 & & 0,30 & & 0,33 & & 0,30 & & 0,33 & & 0,31 & & 0,29 & & 0,32 & & 0,32 & \\
\hline 0,28 & & 0,28 & & 0,31 & & 0,30 & & 0,31 & & 0,34 & & 0,28 & & 0,32 & & 0,34 & & 0,33 & \\
\hline 0,34 & & 0,29 & & 0,34 & & 0,31 & & 0,34 & & 0,30 & & 0,33 & & 0,30 & & 0,31 & & 0,29 & \\
\hline 0,30 & & 0,32 & & 0,29 & חמ & 0,34 & & 0,33 & & 0,33 & & 0,34 & & 0,28 & 20 & 0,28 & & 0,34 & \\
\hline 0,31 & & 0,31 & 19 & 0,30 & 20 & 0,35 & 22 & 0,35 & 21 & 0,35 & 17 & 0,33 & 19 & 0,30 & 20 & 0,29 & 20 & 0,31 & 10 \\
\hline 0,35 & & 0,31 & & 0,31 & & 0,30 & & 0,31 & & 0,28 & & 0,28 & & 0,28 & & 0,31 & & 0,29 & \\
\hline 0,29 & & 0,28 & & 0,31 & & 0,28 & & 0,34 & & 0,28 & & 0,28 & & 0,33 & & 0,33 & & 0,34 & \\
\hline 0,27 & & 0,29 & & 0,31 & & 0,30 & & 0,30 & & 0,31 & & 0,30 & & 0,29 & & 0,32 & & 0,30 & \\
\hline 0,28 & & 0,34 & & 0,32 & & 0,27 & & 0,28 & & 0,30 & & 0,29 & & 0,32 & & 0,35 & & 0,30 & \\
\hline
\end{tabular}

\begin{tabular}{|c|c|c|c|c|c|c|c|c|c|c|c|c|c|c|c|c|c|c|c|}
\hline \multicolumn{20}{|c|}{$U A S$-lint $; N p^{P 6}, b t l-G a l 4 / N p^{C 2}$} \\
\hline \multicolumn{2}{|c|}{ E1 } & \multicolumn{2}{|c|}{ E2 } & \multicolumn{2}{|c|}{ E3 } & \multicolumn{2}{|c|}{ E4 } & \multicolumn{2}{|c|}{ E5 } & \multicolumn{2}{|c|}{ E6 } & \multicolumn{2}{|c|}{ E7 } & \multicolumn{2}{|c|}{ E8 } & \multicolumn{2}{|c|}{ E9 } & \multicolumn{2}{|c|}{ E10 } \\
\hline $\mathrm{D}$ & $\mathrm{F}$ & $\mathrm{D}$ & $\mathrm{F}$ & $\mathrm{D}$ & $\mathrm{F}$ & $\mathrm{D}$ & $\mathrm{F}$ & $\mathrm{D}$ & $\mathrm{F}$ & $\mathrm{D}$ & $\mathrm{F}$ & $\mathrm{D}$ & $\mathrm{F}$ & $\mathrm{D}$ & $\mathrm{F}$ & $\mathrm{D}$ & $\mathrm{F}$ & $\mathrm{D}$ & $\mathrm{F}$ \\
\hline 0,63 & \multirow{10}{*}{11} & 0,44 & \multirow{10}{*}{9} & 0,51 & \multirow{10}{*}{8} & 0,70 & \multirow{10}{*}{11} & 0,35 & \multirow{10}{*}{11} & 0,42 & \multirow{10}{*}{13} & 0,33 & \multirow{10}{*}{10} & 0,33 & \multirow{10}{*}{13} & 0,43 & \multirow{10}{*}{12} & 0,69 & \multirow{10}{*}{8} \\
\hline 0,51 & & 0,42 & & 0,39 & & 0,31 & & 0,34 & & 0,46 & & 0,57 & & 0,28 & & 0,71 & & 0,67 & \\
\hline 0,63 & & 0,40 & & 0,71 & & 0,30 & & 0,33 & & 0,56 & & 0,28 & & 0,44 & & 0,70 & & 0,70 & \\
\hline 0,54 & & 0,29 & & 0,66 & & 0,44 & & 0,64 & & 0,35 & & 0,29 & & 0,63 & & 0,32 & & 0,30 & \\
\hline 0,62 & & 0,30 & & 0,47 & & 0,33 & & 0,35 & & 0,30 & & 0,30 & & 0,70 & & 0,39 & & 0,29 & \\
\hline 0,44 & & 0,28 & & 0,59 & & 0,34 & & 0,63 & & 0,68 & & 0,30 & & 0,32 & & 0,37 & & 0,28 & \\
\hline 0,30 & & 0,30 & & 0,34 & & 0,35 & & 0,48 & & 0,50 & & 0,29 & & 0,34 & & 0,41 & & 0,27 & \\
\hline 0,34 & & 0,78 & & 0,45 & & 0,58 & & 0,37 & & 0,40 & & 0,74 & & 0,70 & & 0,69 & & 0,76 & \\
\hline 0,70 & & 0,75 & & 0,62 & & 0,29 & & 0,58 & & 0,37 & & 0,68 & & 0,29 & & 0,37 & & 0,78 & \\
\hline 0,53 & & 0,74 & & 0,42 & & 0,28 & & 0,71 & & 0,66 & & 0,73 & & 0,64 & & 0,43 & & 0,69 & \\
\hline
\end{tabular}




\begin{tabular}{|l|l|l|l|l|l|l|l|l|l|l|l|l|l|l|l|}
\hline 0,67 \\
\hline 0,46
\end{tabular}

For method details of measurements see chapter 4.3.11. 


\section{Acknowledgements}

First of all, I want to thank Prof. Dr. Reinhard Schuh for giving me the opportunity to carry out my $\mathrm{PhD}$ studies in his research group. He provided amazing mentoring and was always available for discussions or answering questions. Furthermore, he provided an unprecedented supply of cake which supported the experimental work. I also want to thank Prof. Dr. Jörg Großhans and Prof. Dr. Ernst Wimmer for participating in my thesis committee and providing helpful advice and discussions.

I want to thank Prof. Dr. Herbert Jäckle for providing a stimulating environment in his department and for taking care that all members of the department are working in "Eintracht". I also want to thank Dr. Gerd Vorbrüggen and Dr. Ralf Pflanz for helpful discussions, and Sharif Mahsur for great support in cell culture experiments. Furthermore, I want to thank all former and current members of the research group Molecular Organogenesis for helpful advice, coffee breaks, field trips to exotic animals and technical help in experiments.

I am especially thankful to Jasmin Rehman for her great support and also for her help in any microscopy related questions. Last but not least, I want to thank my daughter Charlotte for providing joy, fun, scientific advice, and the best distractions from work. 